\title{
The genus Macroteleia Westwood (Hymenoptera, Platygastridae s. I., Scelioninae) from China
} \author{
Canada, K.W. Neatby Building, Ottawa, Ontario K1A 0C6, Canada \\ † urn:lsid:zoobank.org:author:CDB89961-BBC3-412B-BE7F-B3B9E290B991 \\ † urn:lsid:zoobank.org:author:3508C4FF-F027-445F-8417-90AB4AB8FEOD \\ § urn:lsid:zoobank.org:author:FA505310-F606-4F6C-A1DF-74B9A0055B2E \\ | urn:lsid:zoobank.org:author:A2A78F02-B9EC-46F7-AEB3-EC5F819CC117 \\ Corresponding author: Zai-fu Xu (xuzaifu@scau.edu.cn)
}

Hua-yan Chen ${ }^{1, \dagger}$, Norman F. Johnson ${ }^{2, \neq}$, Lubomír Masner ${ }^{3, \S}$, Zai-fu Xu ${ }^{1,1}$

I Department of Entomology, College of Natural Resources and Environment, South China Agricultural University, Guangzhou 510640, P. R. China 2 Department of Evolution, Ecology and Organismal Biology, The Ohio State University, 1315 Kinnear Road, Columbus, Ohio 43212, U.S.A. 3 Agriculture and Agri-Food

Academic editor: M. Engel | Received 1 March 2012 | Accepted 7 May 2013 | Published 15 May 2013

urn:lsid:zoobank.org:pub:5CF96900-B69B-4F61-9F6F-D0A03DE56BBF

Citation: Chen H-y, Johnson NF, Masner L, Xu Z-f (2013) The genus Macroteleia Westwood (Hymenoptera, Platygastridae s. l., Scelioninae) from China. ZooKeys 300: 1-98. doi: 10.3897/zookeys.300.4934

\begin{abstract}
The genus Macroteleia Westwood (Hymenoptera: Platygastridae s. l., Scelioninae) from China is revised. Seventeen species are recognized based on 502 specimens, all of which are new records for China. Seven new species are described: M. carinigena sp. n. (China), M. flava sp. n. (China), M. gracilis sp. n. (China), M. salebrosa sp. n. (China), M. semicircula sp. n. (China), M. spinitibia sp. n. (China) and M. striatipleuron sp. n. (China). Ten species are redescribed: M. boriviliensis Saraswat (China, India, Thailand), M. crawfordi Kiefer, stat. n. (China, Philippines, Thailand, Vietnam), M. dolichopa Sharma (China, India, Vietnam), M. emarginata Dodd (China, Malaysia), M. indica Saraswat \& Sharma (China, India, Vietnam), M. lamba Saraswat \& Sharma (China, India, Thailand, Vietnam), M. livingstoni Saraswat (China, India), M. peliades Kozlov \& Lê (China, Vietnam), M. rufa Szelényi (China, Egypt, Georgia, Russia, Thailand, Ukraine) and M. striativentris Crawford (China, Philippines, Thailand, Vietnam). The following five new synonyms are proposed: $M$. crates Kozlov \& Lê syn. n. and $M$. demades Kozlov \& Lê syn. n. of M. crawfordi Kieffer; $M$. cebes Kozlov \& Lê syn. n. and $M$. dones Kozlov \& Lê syn. n. of $M$. indica Saraswat \& Sharma; $M$. dores Kozlov \& Lê syn. n. of $M$. lamba Saraswat \& Sharma. A key to the Chinese species of the genus is provided.
\end{abstract}

\section{Keywords}

Platygastridae s. l., Scelioninae, egg parasitoid, key

Copyright Hua-yan Chen et al. This is an open access article distributed under the terms of the Creative Commons Attribution License 3.0 (CC-BY), which permits unrestricted use, distribution, and reproduction in any medium, provided the original author and source are credited. 


\section{Contents}

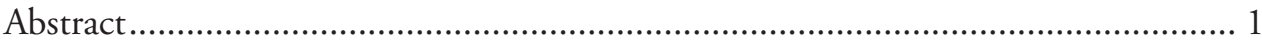

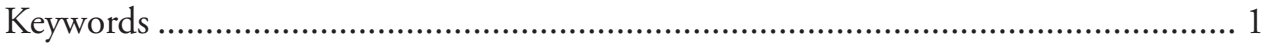

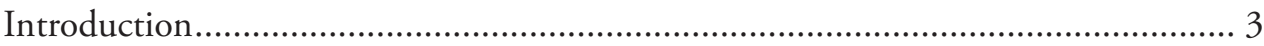

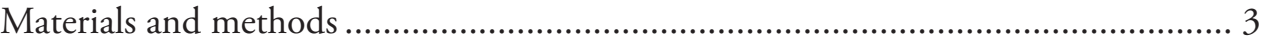

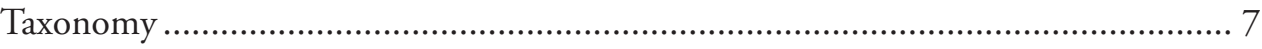

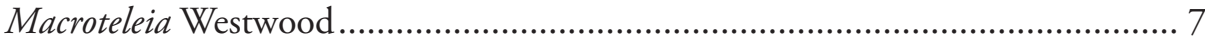

Key to separate Macroteleia, Triteleia and Habroteleia ................................. 11

Key to species of the genus Macroteleia from China ................................... 11

Macroteleia boriviliensis Saraswat .............................................................. 14

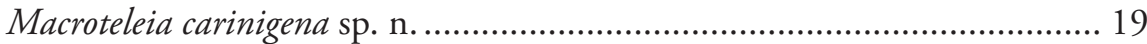

Macroteleia crawfordi Kieffer, stat. n. ........................................................ 22

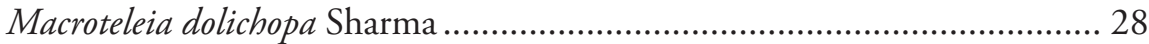

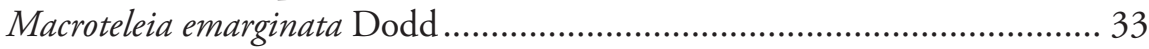

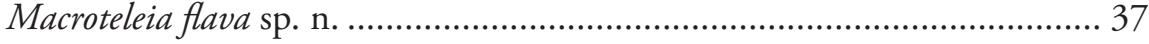

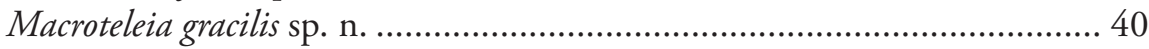

Macroteleia indica Saraswat \& Sharma ....................................................... 43

Macroteleia lamba Saraswat \& Sharma .................................................... 52

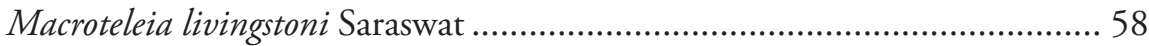

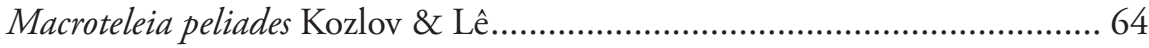

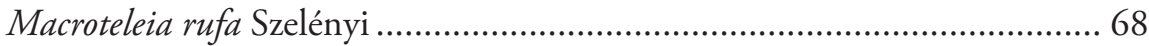

Macroteleia salebrosa sp. n..................................................................... 71

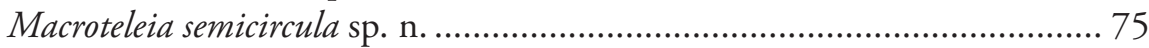

Macroteleia spinitibia sp. n. .................................................................... 79

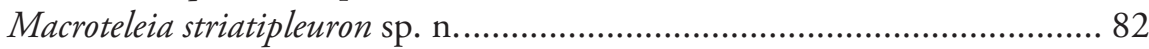

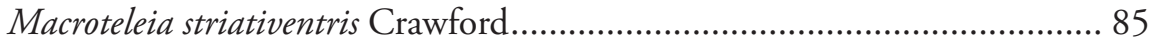

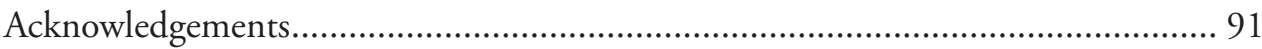

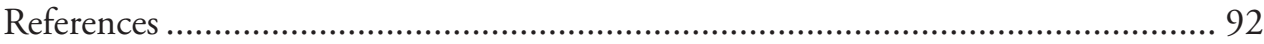

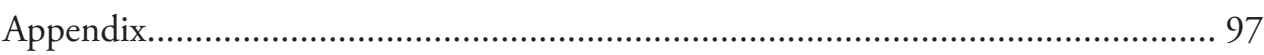




\section{Introduction}

Macroteleia Westwood is a cosmopolitan genus in the subfamily Scelioninae, comprising about 131 described species worldwide (including 2 fossil species; Johnson 2012). Although Macroteleia are found on every continent except Antarctica, they are centred in the tropics and subtropics (Masner 1976). In the past century since Kieffer's (1926) comprehensive study of this genus, several regional revisions have done for the New World (Muesebeck 1977), Australia (Galloway 1978), Vietnam (Lê 2000) and the Palearctic region (Kozlov and Kononova 1990; Kononova and Petrov 2003; Kononova and Kozlov 2008). In Asia, Macroteleia has been recorded from the Philippines, Borneo, Mongolia, India, Vietnam, Japan, Israel, Kazakhstan, Tajikistan and China (Dodd 1920; Kieffer 1926; Szabó 1973; Saraswat and Sharma 1978; Lê 2000; Kononova and Kozlov 2008; Johnson 2012). No species have been recorded formally from China.

Available host data suggest that species of Macroteleia are parasitoids of eggs of longhorned grasshoppers (Orthoptera: Tettigoniidae). In the original description of $M$. virginiensis, Ashmead (1893) mentioned that the specimens were reared from eggs of Orchelimum glaberrimum (Burmeister) (Orthoptera: Tettigoniidae), which was later considered to be O. erythrocephalum Davis (Muesebeck 1977). Morgan (1901) reported that the eggs of $O$. agile (De Geer) were parasitized by a Macroteleia species near M. floridana (Ashmead). In the original description of $M$. surfacei, Brues (1907) asserted that the specimens were reared during May from eggs of a "locustid". Cole (1931) also listed Macroteleia species near M. floridana (Ashmead) as a parasite of Conocephalus sp. (Orthoptera: Tettigoniidae). Priesner (1951) supposed that M. eremicola Priesner, which was later considered to be a junior synonym of M. rufa Szelényi (Kononova and Kozlov 2008), parasitized eggs of Tettigoniidae based on the collecting habitat (halfa grass and sugar cane). In his revision of the New World Macroteleia, Muesebeck (1977) stated that M. secreta Muesebeck and M. pilosa Muesebeck were reared from eggs of Tettigoniidae, and some specimens of $M$. punctulata Kieffer and M. macrogaster Ashmead were reared from eggs of Bucrates capitatus (De Geer) (Orthoptera: Tettigoniidae) and Orchelimum sp., respectively.

During recent years, we have accumulated many specimens of Platygastridae $s . l$. during surveys of Chinese Hymenoptera. Among them, seventeen species of Macroteleia are recognized here, of which seven species are considered to be new to science and a total of 17 species are newly recorded from China. All the Chinese Macroteleia species are described and keyed and 6 new synonyms are proposed.

\section{Materials and methods}

This work is based upon specimens deposited in the following collections, with abbreviations used in the text: IEBR, Institute of Ecology and Biological Resources, Hanoi, Vietnam; RABC, personal collection of R. A. Beaver, Chiang Mai, Thailand; SCAU, South China Agricultural University, Guangzhou, China; USNM, National Museum of Natural History, Washington, DC. 


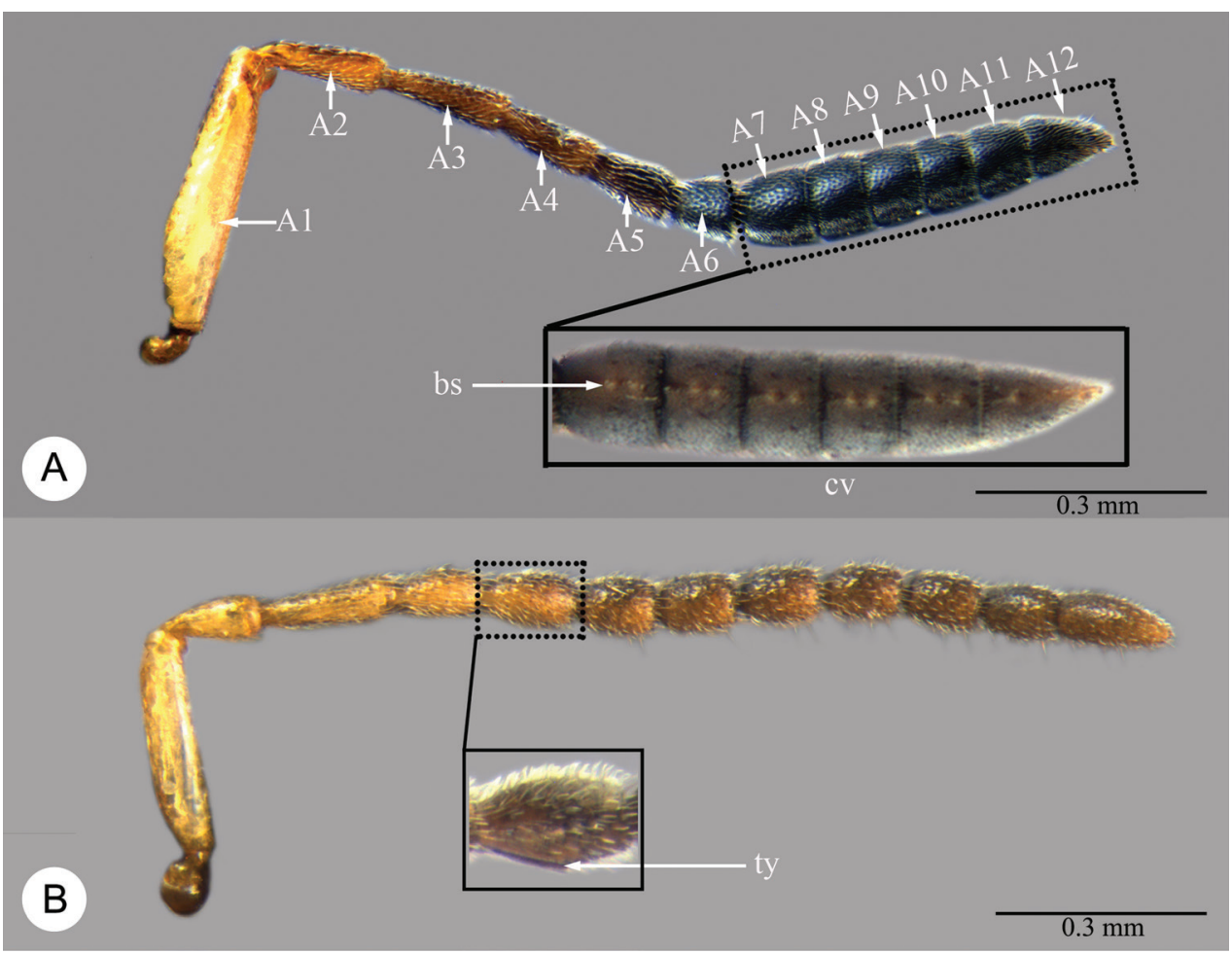

Plate I. A Macroteleia striatipleuron sp. n., Antenna, female B Macroteleia carinigena sp. n., Antenna, male. A1, A2, ... A12: antennomeres 1-12; bs: basiconic sensillum; cv: clava; ty: tyloid.

Abbreviations and morphological terms used in text: A1, A2, ... A12 (Plate 1): antennomere 1, 2, .. 12; LOL (Plate 2B): lateral ocellar line, shortest distance between inner margins of median and lateral ocelli (Masner 1980); OOL (Plate 2B): ocular ocellar line, shortest distance from inner orbit and outer margin of posterior ocellus (Masner 1980); POL (Plate 2B): posterior ocellar line, shortest distance between inner margins of posterior ocelli (Masner 1980); T1, T2, ... T7: metasomal tergite 1, 2, .. 7 (Plates 5, 7); S1, S2, .. S7: metasomal sternite 1, 2, .. 7 (Plates 5, 7). Morphological terminology otherwise generally follows Masner (1980) and Mikó et al. (2007); the following are illustrated and labeled to aid in the description of Macroteleia. Central keel (ck: Plate 2A); Clypeus (cl: Plate 2A); Gena (ge: Plate 2A); Cervical pronotal area (cpa: Plate 3B); Dorsal pronotal area (dpa: Plate 3B); Lateral lobe of mesoscutum (llm: Plate 3A); Longitudinal median sternal carina (lmc: Plate 5C); Lower mesepisternum (lmes: Plate 3B); Labial palpus (lp: Plate 2A); Lateral pronotal area (lpa: Plate 3B); Mandible (md: Plate 2A); Mesopleural depression (med: Plate 3B); Metapleuron (mep: Plate 3B); Middle lobe of mesoscutum (mlm: Plate 3A); Maxillary palpus (mp: Plate 2A); Metascutellum (msct: Plate 3A); Netrion (net: Plate 3B); Notauli (not: Plate 3A); Occipital carina (oc: Plate 2B); Ocellar triangle (ot: Plate 2B); Propodeal lobe (pl: Plate 3A); Sublateral tergal carina (stc: Plate 5A); Transverse sulcus of T2 (trs: 


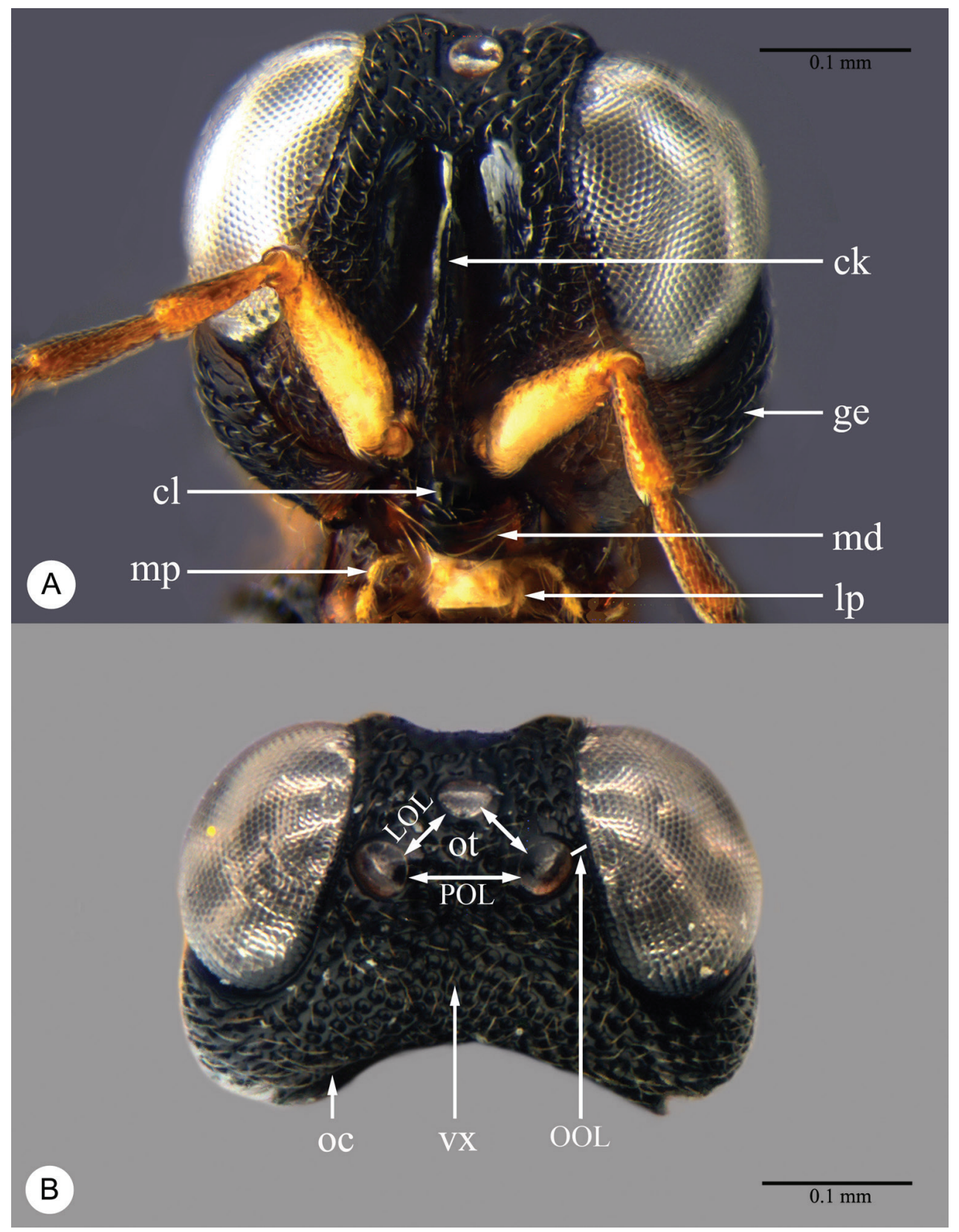

Plate 2. Macroteleia striatipleuron sp. n., female. A Head, anterior view B Head, dorsal view. ck: central keel; cl: clypeus; ge: gena; LOL: lateral ocellar line; lp: labial palpus; md: mandible; mp: maxillary palpus; oc: occipital carina; OOL: ocular ocellar line; ot: ocellar triangle; POL: posterior ocellar line; vx: vertex.

Plate 5A); Upper mesepisternum (umes: Plate 3B); Vertex (vx: Plate 2B). Appendix I lists terms associated with identifiers in the Hymenoptera Anatomy Ontology (Yoder et al. 2010). 


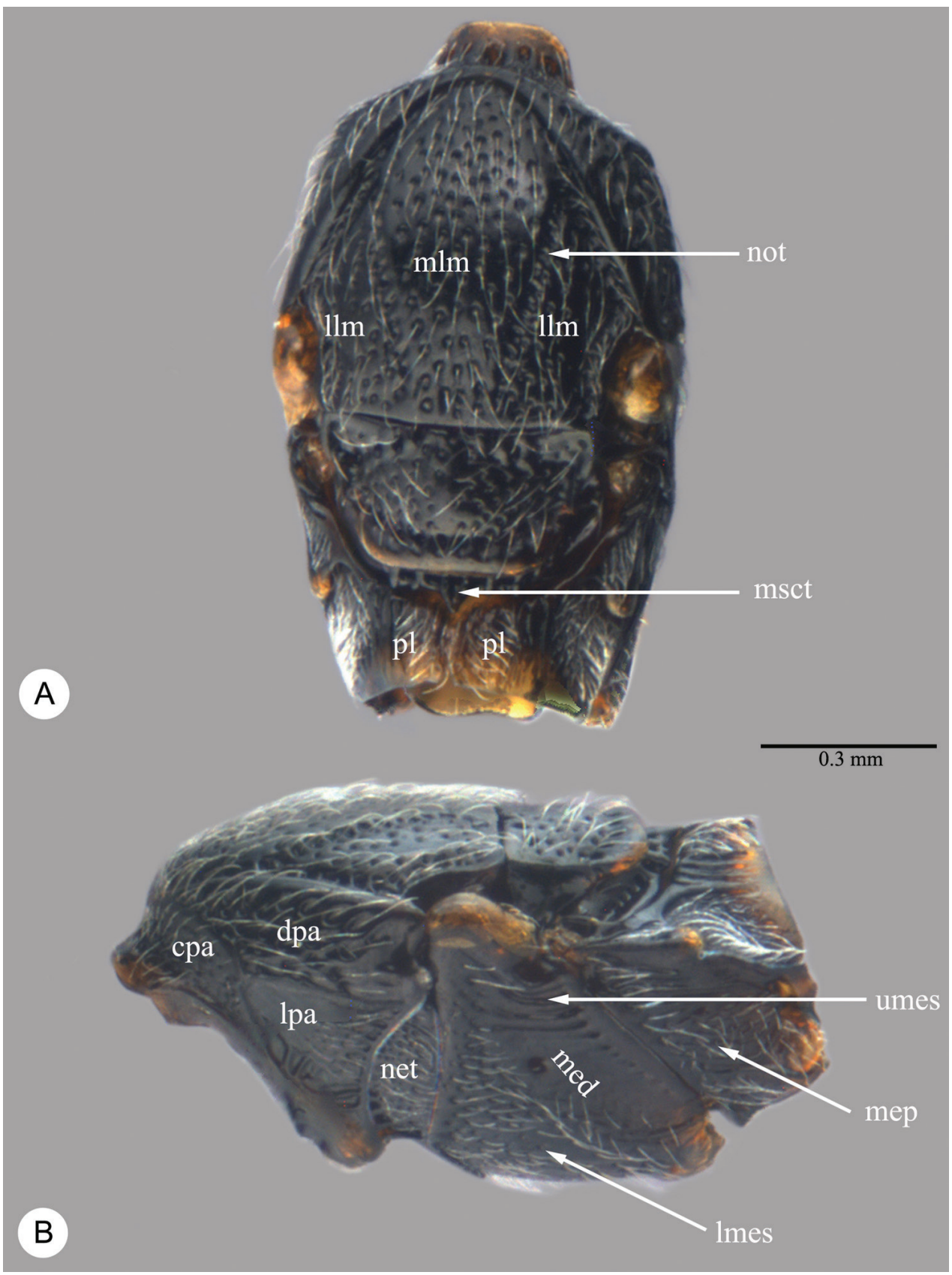

Plate 3. Macroteleia striativentris Crawford, female. A Mesosoma, dorsal view B Mesosoma, lateral view. cpa: cervical pronotal area; dpa: dorsal pronotal area; lpa: lateral pronotal area; llm: lateral lobe of mesoscutum; lmes: lower mesepisternum; med: mesopleural depression; mep: metapleuron; mlm: middle lobe of mesoscutum; msct: metascutellum; net: netrion; not: notauli; pl: propodeal lobe; umes: upper mesepisternum. 
Descriptions and measurements were made under a stereomicroscope (Olympus SZ61). Images were processed with a Photometrics CoolSNAP Camera attached to a stereomicroscope (Zeiss Stemi 2000-CS) and Image Pro Plus version 6.0 software and figures were post-processed with Adobe Photoshop CS3 Extended.

\section{Taxonomy}

\section{Macroteleia Westwood}

http://species-id.net/wiki/Macroteleia

Macroteleia Westwood 1835: 70. Original description. Type: Macroteleia cleonymoides Westwood, by monotypy. Brullé 1846: 621 (description); Ashmead 1893: 209, 210, 211, 216 (description, keyed, key to species of U.S. and Canada); Ashmead 1894: 216, 222 (key to species of St. Vincent, keyed); Dalla Torre 1898: 501 (catalog of species); Ashmead 1900: 327 (list of species of West Indies); Ashmead 1903: 91, 92, 93, 94 (keyed); Brues 1907: 154 (key to species of United States); Brues 1908: 27, 28, 34, 51 (diagnosis, list of species, keyed); Kieffer 1908a: 22 (key to new species described); Kieffer 1908b: 122, 170 (key to species, keyed); Kieffer 1910a: 316 (key to species of Brazil); Kieffer 1910b: 66, 89 (description, list of species, keyed); Kieffer 1912: 59 (key to species of Seychelles); Dodd 1913a: 131, 151 (key to species of Australia, keyed); Dodd 1913b: 176 (comparison with Ceratoteleia Kieffer); Kieffer 1913a: 232 (description); Kieffer 1913b: 323 (key to species of the Philippines); Kieffer 1914a: 312 (description, key to species of Europe and Algeria); Kieffer 1914b: 298 (key to species of the Philippines); Dodd 1915: 12 (key to species of Australia); Kieffer 1917: 55 (key to species of the Philippines); Kieffer 1926: 273, 520 (description, keyed, key to species); Nixon 1931: 367 (keyed, key to species of Africa); Dodd 1933: 75 (comparison with Prosapegus Kieffer key to species of Australia); Nixon 1933: 292 (keyed); Maneval 1940: 114 (keyed); Risbec 1950: 598 (key to species of Africa); Muesebeck and Walkley 1951: 706 (catalog of species of U.S. and Canada); Muesebeck and Walkley 1956: 367 (citation of type species); Masner 1964: 137 (description); Baltazar 1966: 183 (catalog of species of the Philippines); Szabó 1966: 421 (key to species of Hungary known to the author); De Santis 1967: 226 (catalog of species of Argentina); Muesebeck and Walkley 1967: 299 (second supplement to Muesebeck and Walkley (1951)); Kozlov 1971: 40 (keyed); Masner 1976: 27 (key to Macroteleia Westwood and Triteleia Kieffer); Muesebeck 1977: 1 (description, key to species of the New World); Galloway 1978: 298 (description, key to species of Australia); Kozlov 1978: 614 (key to species of the European USSR); Muesebeck 1979: 1153 (catalog of species of U.S. and Canada); De Santis 1980: 313 (catalog of species of Brazil); Mani and Sharma 1982: 168 (description, key to species of India); Saraswat 
1982: 343 (key to species of India); Galloway and Austin 1984: 9, 14 (list of species described from Australia, keyed); Kozlov and Kononova 1987: 93 (key to species of Palearctic region); Kozlov and Kononova 1990: 95, 173, 188, 189 (description, key to species of Palearctic, keyed); Carpenter 1992: 471 (fossil references); Johnson 1992: 423(catalog of world species); Kononova 1995: 61, 69 (keyed, diagnosis, key to species of Russian Far East); Austin and Field 1997: 22, 689 (structure of ovipositor system, discussion of phylogenetic relationships); Lê 2000: 31, 52 (keyed, description, key to species); Loiácono \& Margaría 2002: 557 (catalog of Brazilian species); Kononova and Petrov 2003: 604, 605 (description, key to species of Palearctic region); Rajmohana K 2006: 117, 125 (description, keyed); Kononova and Kozlov 2008: 22, 230, 231 (description, keyed, key to species of Palearctic region); Popovici and Johnson 2012: 380 (description of internal genitalia).

Baeoneura Förster 1856: 100, 102. Original description. Type: Baeoneura floridana Ashmead, designated by Muesebeck and Walkley (1956). Howard 1886: 172 (keyed); Cresson 1887: 83, 313 (keyed, catalog of species of U.S. and Canada); Provancher 1888: 402 (description); Ashmead 1893: 210, 211, 234 (description, keyed); Ashmead 1894: 217 (keyed); Dalla Torre 1898: 498 (catalog of species); Ashmead 1903: 92, 94 (keyed); Brues 1908: 27, 28, 39 (diagnosis, list of species, keyed); Kieffer 1908b: 115 (keyed); Kieffer 1910b: 63, 72 (description, list of species, keyed); Kieffer 1913a: 223, 235 (description); Kieffer 1926: 267, 351 (description, keyed); Maneval 1940: 112 (keyed); Muesebeck and Walkley 1956: 335 (junior synonym of Macroteleia Westwood).

Macrotelia Förster 1856: 105 (diagnosis, spelling error).

Parapegus Kieffer 1908b: 149. Original description. Type: Apegus (parapegus) punctatus Kieffer, designated by Kieffer (1910b). Kieffer 1913a: 232 (description); Kieffer 1914a: 306 (description, key to species of Europe and Algeria); Kieffer 1926: 273, 497 (description, keyed, key to species); Maneval 1940: 114 (keyed); Masner 1956: 235 (diagnosis, key to species); Muesebeck and Walkley 1956: 380 (citation of type species); Kozlov 1971: 40 (keyed); Masner 1976: 27 (junior synonym of Macroteleia Westwood); Kozlov 1978: 616 (key to species of the European USSR).

Prosapegus Kieffer 1908b: 121, 147. Original description. Type: Anteris elongata Ashmead, by monotypy and original designation, keyed. Brues 1908: 50 (diagnosis, list of species); Kieffer 1910b: 65, 85, 86 (description, list of species, keyed); Kieffer 1913a: 232 (description); Dodd 1920: 321 (diagnosis, taxonomic status); Kieffer 1926: 272, 488 (description, keyed); Dodd 1933: 75, 81 (comparison with Macroteleia Westwood, key to species of Australia); Muesebeck and Walkley 1951: 705 (catalog of species of U.S. and Canada); Muesebeck and Walkley 1956: 391 (citation of type species); Masner 1964: 137 (junior synonym of Macroteleia Westwood).

Stictoteleia Kieffer 1926: 272, 546. Original description. Type: Macroteleia virginiensis Ashmead, by original designation, keyed. Muesebeck and Walkley 1951: 706 (catalog of species of U.S. and Canada); Muesebeck and Walkley 1956: 400 (citation of type species); Masner 1964: 137 (junior synonym of Macroteleia Westwood). 


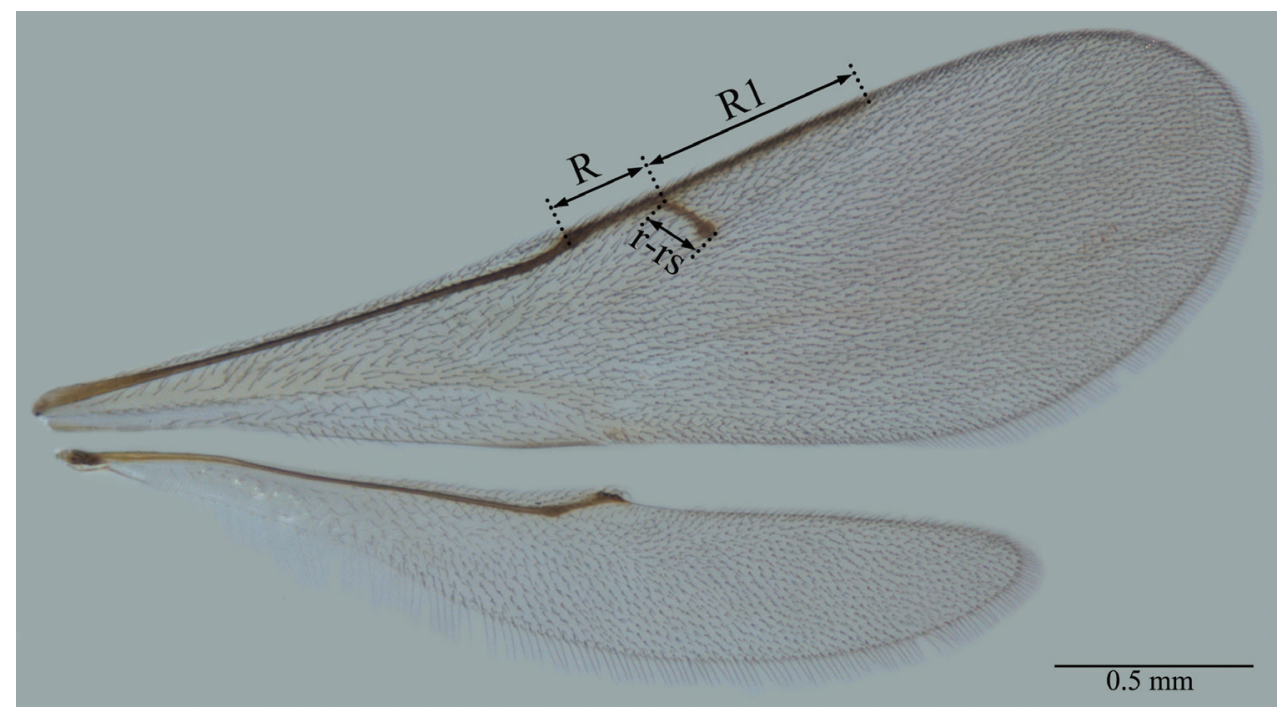

Plate 4. Macroteleia striativentris Crawford, fore and hind wing, female. R: marginal vein; R1: postmarginal vein; r-rs: stigmal vein.

Diagnosis. Macroteleia is known as one of the longest and often the largest wasps in the subfamily Scelioninae. It may be distinguished from other genera of the subfamily by the combination of the following characters: marginal vein (R) distinctly elongate, as long as or longer than stigmal vein ( $\mathrm{r}$-rs) (Plate 4); propodeum without armature, divided into two separated halves (Plates 14B, 16D, 17D, 37B, 38D, 40B, $52 \mathrm{~B}, 54 \mathrm{~B}, 57 \mathrm{~B}, 63 \mathrm{~B}$ ) or continuous medially (Plates 3A, 18D, 20B, 23B, 25D, 31D, 32C, 34B, 43B, 45B, 49B, 65B, 66D, 68B); wings hyaline or subhyaline (Plate 4), rarely with infuscations or darkening (Plate 60D); T6 in females strongly compressed laterally to form a wedge (Plate $5 \mathrm{~B}$ ); apex of $\mathrm{T} 7$ in males differentiated, truncate (Plates 6A, 8D, 10D, 12D, 35B, 47F, 50B, 55B, 58B, 61B) or excavate medially in New World species, pointed medially (Plates 6C, 15B, 21B, 24B, 26B, 28E, 41B, 46B, 69B) or cut off in Old World species, but never bidentate or bispinose (Masner 1976; Muesebeck 1977).

Comments. Macroteleia is mostly similar to Triteleia that found in China both in size and body shape. Masner (1976) mentioned that the two genera were often confused, but Triteleia can be distinguished by the fact that T6 in females strongly depressed dorsoventrally to form a flat triangle, often spined at the apex; T7 in males armed posterolaterally with 2 sharp spikes or at least tyiny points; propodeum often armed dorsally, protruded into teeth or at least dorsal points or forms a triangular protuberance. Habroteleia is also similar to Macroteleia, but can be distinguished by having postmarginal vein (R1) absent or only rudimentary; propodeum spined posteromedially and at posterolateral corners; T6 in females strongly depressed dorsoventrally to form a flat triangle as in Triteleia. The following key is used to separate Triteleia and Habroteleia from Macroteleia with the fewest characters possible. 


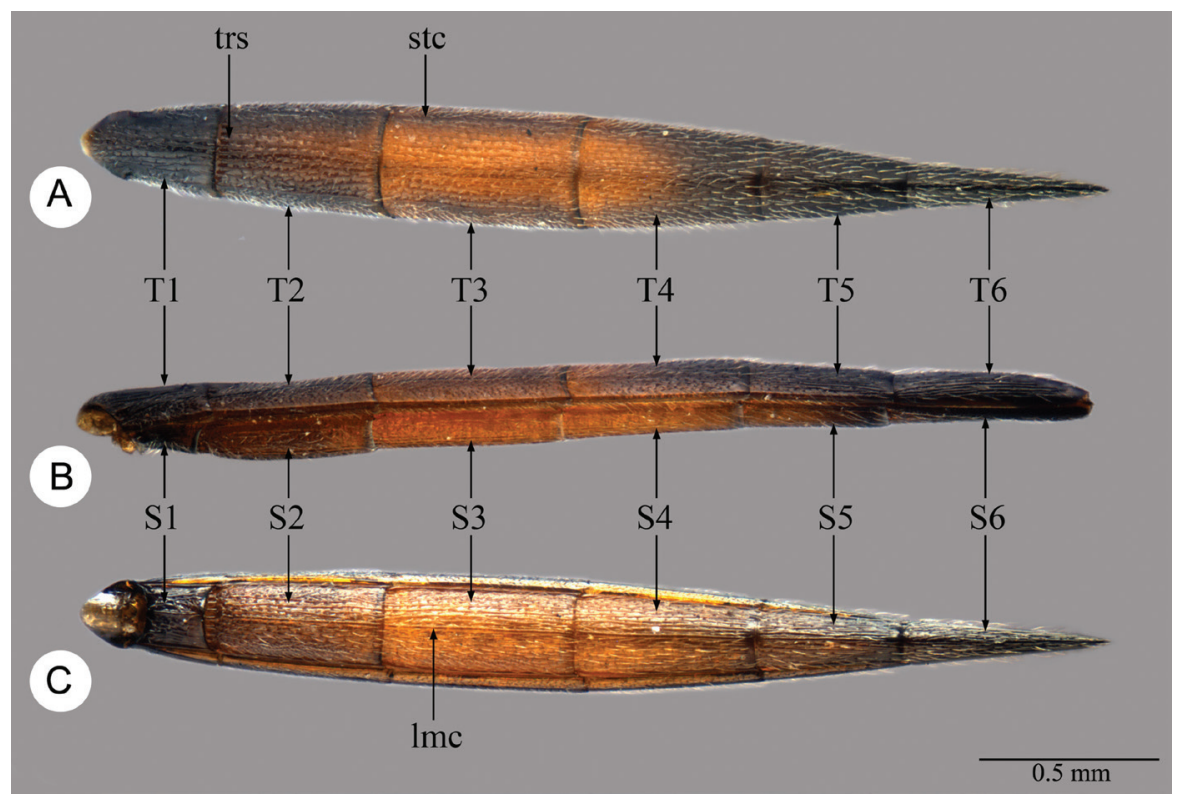

Plate 5. Macroteleia striativentris Crawford, female. A Metasoma, dorsal view B Metasoma, lateral view C Metasoma, ventral view. lmc: longitudinal median sternal carina; S1, S2, .. S6: metasomal sternite 1-6; stc, sublateral tergal carina; trs: transverse sulcus of T2; T1, T2, ... T6: metasomal terga 1-6.

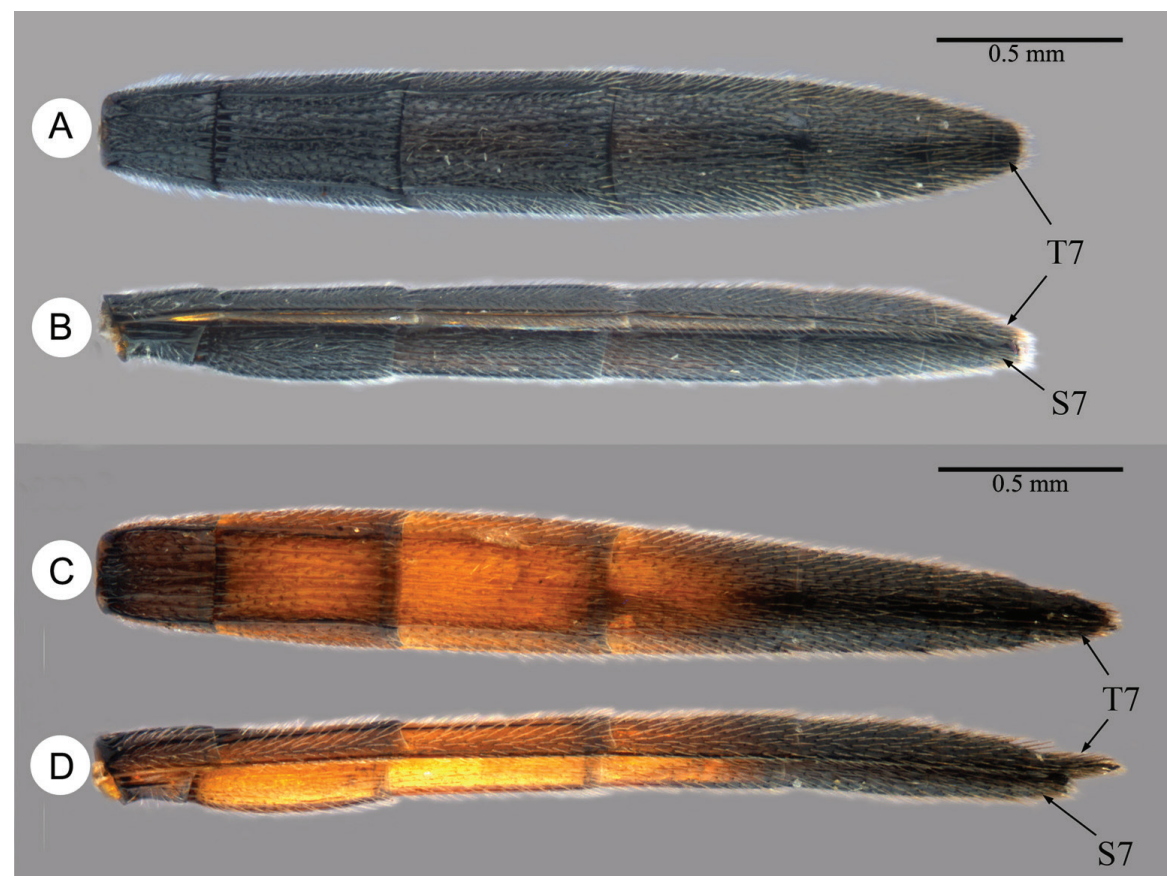

Plate 6. A Macroteleia boriviliensis Saraswat, male, metasoma, dorsal view B Macroteleia boriviliensis Saraswat, male, metasoma, lateral view C Macroteleia striativentris Crawford, male, metasoma, dorsal view D Macroteleia striativentris Crawford, male, metasoma, lateral view. S7: metasomal sternite 7; T7: metasomal tergite 7. 


\section{Key to separate Macroteleia, Triteleia and Habroteleia}

1 Postmarginal vein in fore wing absent; female ovipositor Ceratobaeus-type.. Habroteleia Kieffer

- $\quad$ Postmarginal vein in fore wing well developed, distinctly longer than stigma vein (r-rs); female ovipositor Scelio-type ....................................................2

2 Female T6 strongly compressed, wedge like; male apical tergite apically emarginated or with 1 central spine but never bispinose ....Macroteleia Westwood T6 flatly triangular, not compressed; male apical tergite with postero-lateral corners bispinose or at least pointed.

Triteleia Kieffer

\section{Key to species of the genus Macroteleia from China}

\section{Females}

(Unknown for M. carinigena, M. gracilis, M. boriviliensis and M. spinitibia)

1 Propodeum divided into two separated subtriangular lobes (Plates 14B, 16D, 17D, 37B, 38D, 40B, 52B, 54B, 57B, 63B)

- Propodeum continuous medially, not divided into two separated lobes (Plates 3A, 18D, 20B, 23B, 25D, 31D, 32C, 34B, 43B, 45B, 49B, 65B, $66 \mathrm{D}, 68 \mathrm{~B})$

2 Head and mesosoma variably yellow to dark brown or orange (Plates 52B, 57B)

- $\quad$ Head and mesosoma entirely black (Plates 14B, 37B, 38D, 40B, 54B, 65B, 66D, 68B)

3 Metascutellum triangular, strongly produced medially (Plate 52B); central keel weakly developed above interantennal process (Plate 52A).

Macroteleia rufa Szelényi

Metascutellum semicircular (Plate 57B); central keel well developed, extending onto interantennal process, slightly bifurcating dorsally (Plate 57A)

Macroteleia semicircula sp. $\mathbf{n}$.

4 Central keel well developed, extending onto interantennal process (Plates 2A, 54A); legs robust, hind femur strongly swollen (Plates 53B, 62B) ............... 5 Central keel weakly developed above interantennal process (Plates 14A, 37A, 38C, 40A); legs slender, hind femur weakly swollen (Plates 13B, 17B, 36B, 38B, 39B).

5 Mesopleural depression smooth (Plate 54C); occipital carina interrupted medially (Plate 54B); hind coxa dark brown to nearly black (Plate 54C)

Macroteleia salebrosa sp. $\mathbf{n}$.

- $\quad$ Mesopleural depression longitudinally striate (Plate 63B); occipital carina continuous medially (Plate 2B); hind coxa yellow (Plate 63B) 
Metascutellum tongue-like (Plates 14B, 16D, 17D); metapleuron longitudinally striate dorsally, densely punctate ventrally (Plates $14 \mathrm{C}, 16 \mathrm{E}, 17 \mathrm{E})$.......

Macroteleia crawfordi Kieffer

Metascutellum triangular, strongly produced medially (Plates 37B, 38D, 40B); metapleuron longitudinally striate throughout (Plates 37C, 40C)

Macroteleia lamba Saraswat \& Sharma Head entirely black (Plates 18D, 20B, 23B, 43B, 45B, 49B, 65B, 66D, 68B)

Length of T3 1.11-1.39× length of T6; T5 distinctly wider than long (Plates 30C, 31F, 34D); apex of fore wing extending from as far as mid-length of T5 to base of T6. Macroteleia indica Saraswat \& Sharma Length of T3 $0.93-0.99 \times$ length of T6; T5 distinctly longer than wide (Plate $25 \mathrm{~F})$; apex of fore wing extending as far as posterior third of T4

Macroteleia flava sp. $\mathbf{n}$. Metasoma mixed brown and black (Plates 18D, 20D, 65D, 66F, 68D) ... 10 Metasoma entirely black (Plates 23D, 43D, 45D, 49D) ...................... 11 Frons below median ocellus and vertex densely punctate (Plates 65A, 65B, 66C, 66D, 68A, 68B); metapleuron longitudinally striate (Plates 65C, 66E, 68C); mesosoma black (Plates 65B, 66D, 68B)

Macroteleia striativentris Crawford

- $\quad$ Frons below median ocellus and posterior vertex punctate reticulate (Plates 18C, 18D, 20A, 20B); metapleuron longitudinally striate dorsally, punctate rugulose ventrally (Plate 20C); mesosoma orange (Plates $18 \mathrm{D}, 20 \mathrm{~B}$ )

Macroteleia dolichopa Sharma

11 Ocellar triangle densely punctate; lower mesepisternum densely punctate to punctate rugulose; posterior margin of transverse sulcus on T2 straight (Plate 49D)

Macroteleia peliades Kozlov \& Lê

- $\quad$ Ocellar triangle largely smooth, with scattered punctures; lower mesepisternum variably smooth to punctate rugulose; posterior margin of transverse sulcus on T2 convex (Plates 23D, 43D, 45D) 12

12 Gena punctate rugose; body length 5.48-6.14 mm

Macroteleia emarginata Dodd

Gena punctate reticulate; body length 3.28-4.08 mm

Macroteleia livingstoni Saraswat

\section{Males}

(Unknown for $M$. striatipleuron and $M$. rufa)

T7 transverse, apex truncate (Plates 6A, 8D, 10D, 12D, 35B, 47F, 50B, 55B, 58B, 61B)

- $\quad$ T7 subtriangular, apex pointed (Plates 6C, 15B, 21B, 24B, 26B, 28E, 41B, 46B, 69B) 
2 Central keel absent; legs slender, hind femur weakly swollen

Central keel well developed, extending onto interantennal process; legs robust, hind femur strongly swollen .....

Hind tibia without spines over outer surface (Plates 55A, 58A); mesopleural depression smooth.....

Hind tibia with numerous semi-erect, yellow spines over outer surface (Plate 61D); mesopleural depression longitudinally striate (Plate 60C)

Metascutellum rectangular (Plate 55A); fore wing hyaline throughout.

Macroteleia spinitibia sp. n.

Macroteleia salebrosa sp. $\mathbf{n}$.

Metascutellum semicircular (Plate 58A); fore wing slightly infuscate in basal half. Macroteleia semicircula sp. $\mathbf{n}$. T6 longer than wide (Plates 47F, 50B, 55B); body length 7.00-8.00 mm ....

Macroteleia peliades Kozlov \& Lê T6 wider than long (Plates 8D, 10D, 12D, 35B); body less than $7.00 \mathrm{~mm} 6$ Gena with a strong carina parallel to occipital carina, punctate rugose dorsally (Plate 12B); propodeal lobe without longitudinally carinae.....

Macroteleia carinigena sp. $\mathrm{n}$. Gena without strong carina, punctate rugose throughout (Plates 8B, 10B); propodeal lobe with several irregular longitudinal carinae medially.....

Head and mesosoma black; metapleuron longitudinally striate dorsoventrally, punctate rugulose medially..... Macroteleia boriviliensis Saraswat Head and mesosoma yellow or orange; metapleuron longitudinally striate throughout Macroteleia indica Saraswat \& Sharma Lateral ocellus contiguous with inner orbit of compound eye . .9 Lateral ocellus separated from inner orbit of compound eye .11 T7 sharply pointed medially (Plate 21B); mesosoma orange yellow or brown (Plate 21A) Macroteleia dolichopa Sharma T7 bluntly pointed medially (Plates 26B, 69B); mesosoma entirely black (Plates 41A, 46A). 10 Frons below median ocellus densely punctate, interspaces coriaceous; ocellar triangle coriaceous, with scattered punctures.

Macroteleia lamba Saraswat \& Sharma

Frons below median ocellus sparsely punctate, interspaces smooth; ocellar triangle smooth, with scattered punctures Macroteleia livingstoni Saraswat T6 wider than long (Plate 15B); apex of T7 sharply pointed to form a spine (Plate 15B) Macroteleia crawfordi Kieffer T6 longer than wide (Plates 24B, 26B, 28E, 69B); apex of T7 pointed, but not forming a spine (Plates 24B, 26B, 28E, 69B) 12 Head brown (Plates 26A, 69A) 13 Head entirely black (Plates 24A, 28B) 14 Length of A3 0.83-0.90× length of A2; mesosoma black (Plate 69A) 
- $\quad$ Length of A3 equal to length of A2; mesosoma orange yellow (Plate 26A)....

14 Metapleuron longitudinally striate dorsally, punctate rugulose ventrally; length of T7 1.42-1.87× length of S7 (Plate 24C)..... Macroteleia emarginata Dodd - $\quad$ Metapleuron longitudinally striate throughout; length of T7 2.50-3.22× length of S7 (Plate 28F)

Macroteleia gracilis sp. $\mathbf{n}$.

\section{Macroteleia boriviliensis Saraswat}

http://species-id.net/wiki/Macroteleia_boriviliensis

Plates $7-10$

Macroteleia boriviliensis Saraswat 1982: 344 (original description).

Description. Male. Body length 3.77-5.41 mm ( $\mathrm{n}=20)$.

Color. Body black, metasoma somewhat brownish; mandible dark brown; palpi yellow; legs pale brown, becoming darker distally; A1 brown, A2-A4 dark brown, remainder of antenna black; fore wing subhyaline.

Head. Transverse in dorsal view, $1.30-1.56 \times$ as wide as long, slightly wider than mesosoma; OOL short, 0.13-0.29× minimum ocellar width; POL 1.36-1.58× LOL; occipital carina weakly continuous medially, irregularly punctate; central keel absent (Plates 8C, 10C); medial frons contiguously punctate ventrally, with irregularly shaped smooth area dorsally; frons below median ocellus contiguously punctate; vertex densely punctate, interspaces in part with microsculpture; gena punctate rugose; length of A3 1.00-1.12x length of A2.

Mesosoma. Cervical pronotal area densely punctate; dorsal pronotal area areolate; lateral pronotal area smooth dorsally, punctate rugulose ventrally; netrion punctate rugulose; notaulus distinctly foveolate; middle lobe of mesoscutum densely punctate, becoming denser anteriorly and posteriorly; lateral lobes of mesoscutum irregularly punctate; mesoscutellum densely finely punctate throughout; metascutellum transverse, posterior margin straight, longitudinally carinate (Plates $8 \mathrm{~A}, 10 \mathrm{~A}$ ); propodeum continuous medially (Plates $8 \mathrm{~A}, 10 \mathrm{~A}$ ), not divided into two separated lobes, posterior margin narrowly notched medially, each side with several irregular longitudinal carinae medially, otherwise punctate rugulose, covered by dense, recumbent, white setae; upper mesepisternum with a row of somewhat robust longitudinal carinae below subalar pit; lower mesepisternum longitudinally punctate rugulose; mesopleural depression smooth or finely longitudinally striate (Plates $8 \mathrm{~B}, 10 \mathrm{~B}$ ); metapleuron longitudinally striate dorsoventrally, punctate rugulose medially.

Legs. Slender; hind femur weakly swollen, $3.75-3.95 \times$ as long as its maximum width; hind tibia without spines over outer surface; hind basitarsus $10.83-11.60 \times$ as long as its maximum width.

Wings. Apex of fore wing extending from as far as posterior fifth of $\mathrm{T} 4$ to base of T5; R 1.59-1.94x as long as r-rs, R1 1.91-2.2× length of R. 


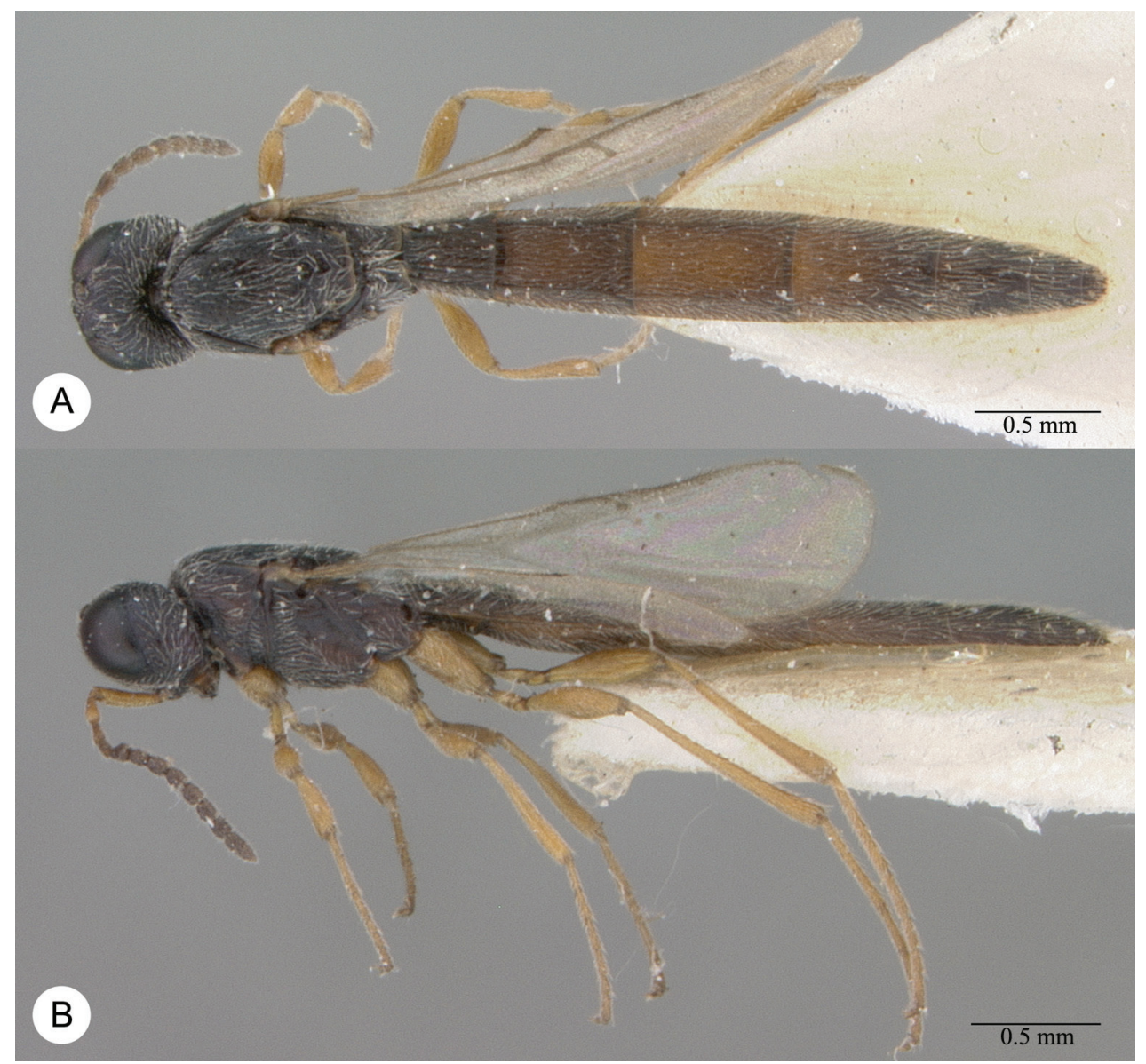

Plate 7. Macroteleia boriviliensis Saraswat, holotype, male. A Dorsal habitus B Lateral habitus.

Metasoma. Posterior margin of transverse sulcus on T2 slightly convex (Plates 8F, 10F); sublateral tergal carinae developed on T1-T4; T1-T4 densely longitudinally striate medially, with numerous delicate punctures in interstices, punctate rugulose laterally; T5-T7 longitudinally punctate rugulose throughout; T6 distinctly wider than long; length of T6 2.33-3.00× length of T7; T7 transverse, apex truncate (Plates $8 \mathrm{D}, 10 \mathrm{D})$; length of T7 $0.64-0.88 \times$ length of S7; S2-S5 sparsely longitudinally striate, with numerous delicate punctures in interstices; $S 6-S 7$ weakly punctate rugulose; prominent longitudinal median carinae present on $\mathrm{S} 2-\mathrm{S} 5$.

Female. Unknown.

Distribution. China (Guangdong, Hainan, Guangxi, Yunnan); Thailand; India. Link to distribution map [http://hol.osu.edu/map-large.html?id=4784].

Material examined. Holotype, ô: INDIA: "School of Entomology, St. John's College, Agra-2, India”, “4-13. Borivili: Bombay, Coll. Mani \& Party, 25.IX.1971”, "Holotype", "Macroteleia boriviliensis Saraswat, ${ }^{\lambda}$ " (deposited in USNM). 


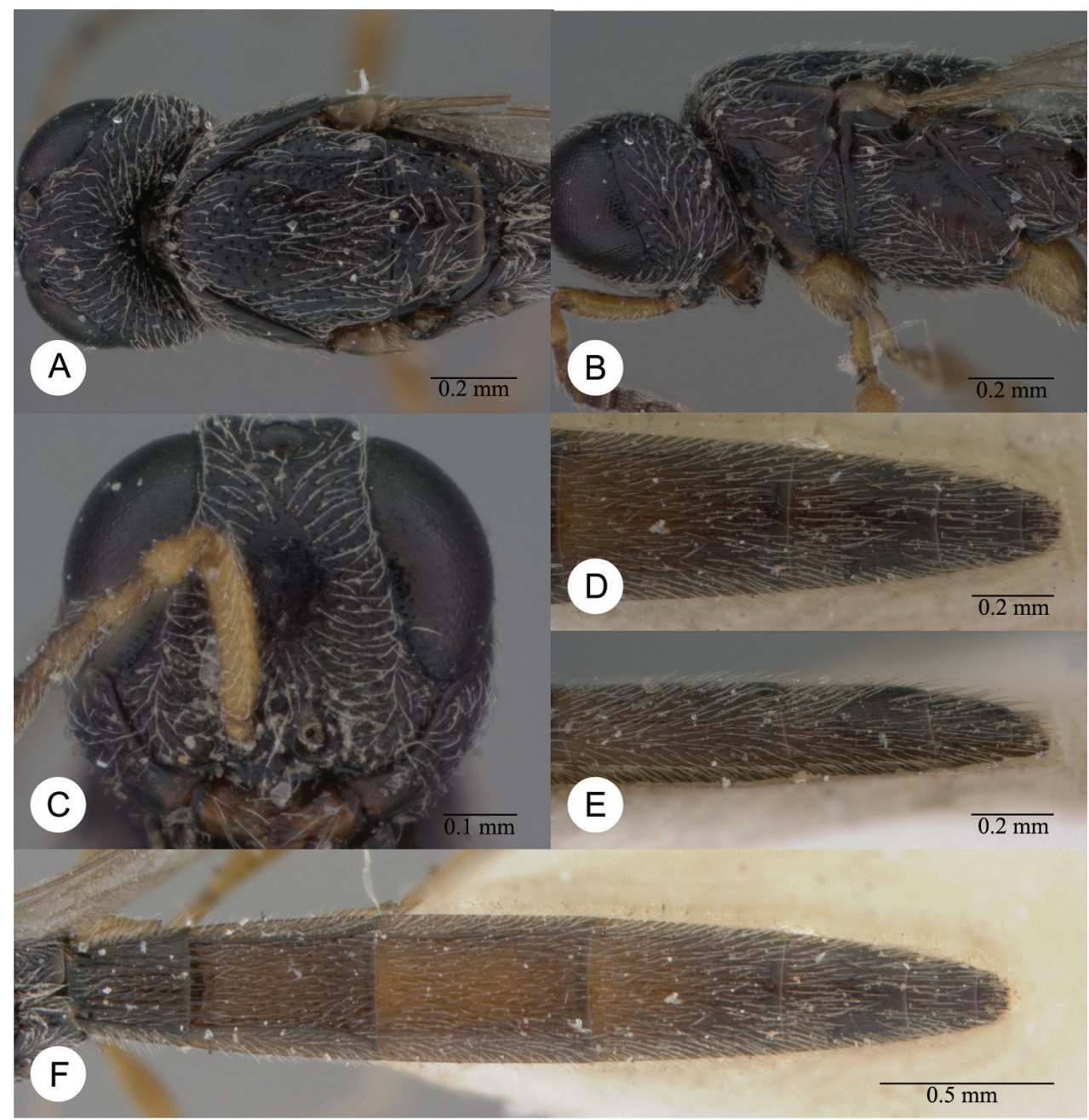

Plate 8. Macroteleia boriviliensis Saraswat, holotype, male. A Head and mesosoma, dorsal view B Head and mesosoma, lateral view $\mathbf{C}$ Head, anterior view D Apex of metasoma, dorsal view E Apex of metasoma, lateral view $\mathbf{F}$ Metasoma, dorsal view.

Other material. CHINA: 1 त, Guangdong, Nanling National Nature Reserve, $24^{\circ} 54^{\prime} \mathrm{N} 113^{\circ} 00^{\prime} \mathrm{E}$, 4.VIII.2004, Jingxian Liu, SCAU 000057 (SCAU); 1 ○, Guang-

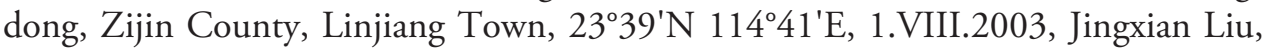

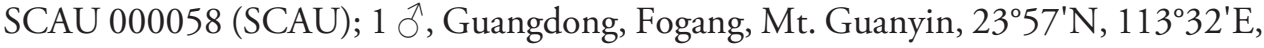

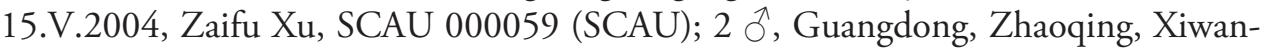
ggu, $23^{\circ} 13^{\prime} \mathrm{N}, 112^{\circ} 31^{\prime} \mathrm{E}, 2-6 . V I I I .2010$, sweeping, Huayan Chen, SCAU 000060, 000061 (SCAU); $1 \mathrm{O}^{\top}$, Guangdong, Zhaoqing, Xiwanggu, $23^{\circ} 13^{\prime} \mathrm{N}, 112^{\circ} 31^{\prime} \mathrm{E}, 2-6$. VIII.2010, yellow pan trap, Huayan Chen, SCAU 000062 (SCAU); 1 \%, Guangdong, Guangzhou, Tianlu Lake, $23^{\circ} 13^{\prime} \mathrm{N}, 113^{\circ} 25^{\prime} \mathrm{E}, 6 . X .2002$, Zaifu Xu, SCAU 000063 (SCAU); $1 \overbrace{}^{\lambda}$, Guangdong, Suixi County, Huanglue Town, $21^{\circ} 20.36^{\prime} \mathrm{N}$, 


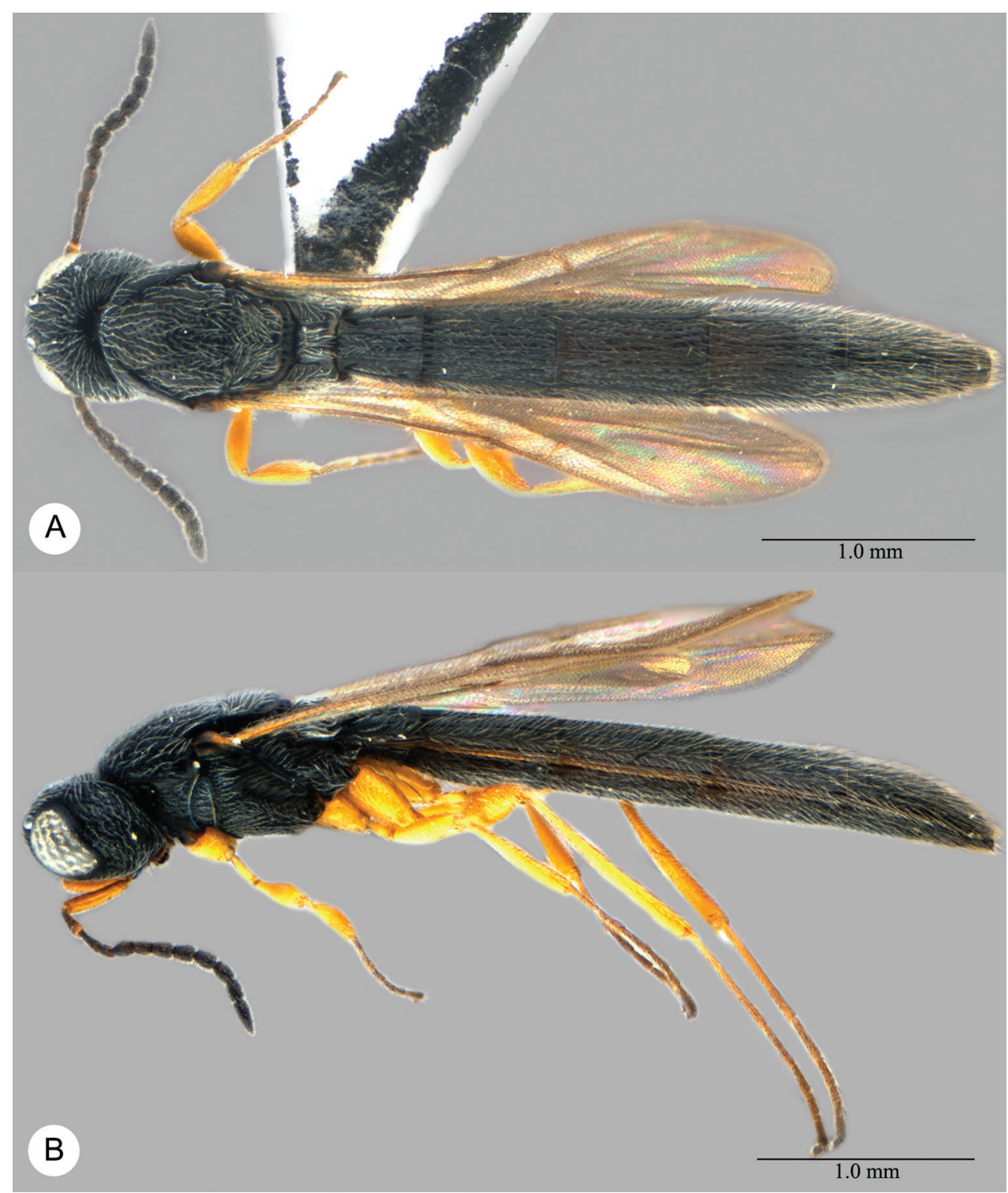

Plate 9. Macroteleia boriviliensis Saraswat, male from Guangdong, Fogang, Mt. Guanyin. A Dorsal habitus B Lateral habitus.

$110^{\circ} 18.61 ' \mathrm{E}, 25 . \mathrm{IX} .2010$, yellow pan trap, Huayan Chen, SCAU 000064 (SCAU); $1 \mathrm{O}^{\top}$, Hainan, Mt. Yinggeling, $18^{\circ} 49^{\prime} \mathrm{N}, 109^{\circ} 11^{\prime} \mathrm{E}, 28 . \mathrm{V} .2007$, Liqiong Weng, SCAU 000065 (SCAU); $1{ }^{\top}$, Hainan, Mt. Yinggeling, $18^{\circ} 49^{\prime} \mathrm{N}, 109^{\circ} 11^{\prime} \mathrm{E}, 17-20 . \mathrm{VII} .2010$, Huayan Chen, SCAU 000066 (SCAU); 1 $\delta$, Hainan, Jianfengling National Nature Reserve, $18^{\circ} 41^{\prime} \mathrm{N}, 108^{\circ} 49^{\prime} \mathrm{E}, 4 . V .2008$, Huayan Chen, SCAU 000067 (SCAU); 1 §̂, Hainan, Mt. Diaoluo, $18^{\circ} 39^{\prime} \mathrm{N} 109^{\circ} 53^{\prime} \mathrm{E}, 28 . V-1 . V I .2007$, Jie Zeng, SCAU 


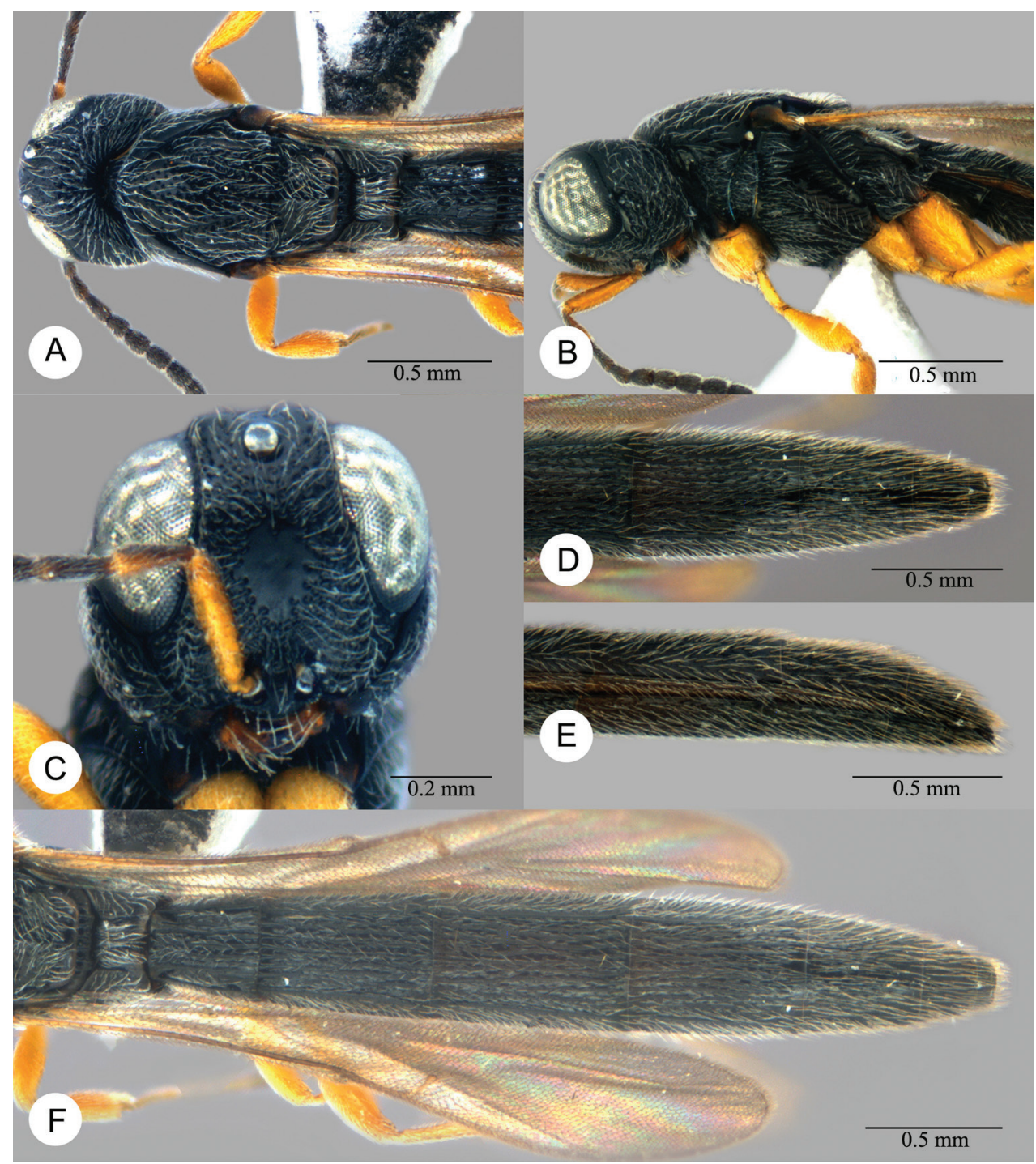

Plate 10. Macroteleia boriviliensis Saraswat, male from Guangdong, Fogang, Mt. Guanyin. A Head and mesosoma, dorsal view B Head and mesosoma, lateral view C Head, anterior view D Apex of metasoma, dorsal view E Apex of metasoma, lateral view F Metasoma, dorsal view.

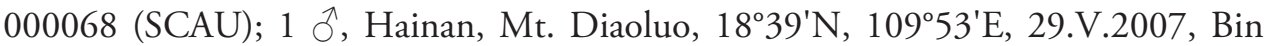
Xiao, SCAU 000069 (SCAU); $1 \hat{\jmath}$, Guangxi, Guilin, Maoershan National Nature Reserve, $25^{\circ} 53^{\prime} \mathrm{N}, 110^{\circ} 25^{\prime} \mathrm{E}, 2-10 . \mathrm{VIII} .2005$, Bin Xiao, SCAU 000070 (SCAU); 4 O’, Yunnan, Gaoligongshan National Nature Reserve, $24^{\circ} 49^{\prime} \mathrm{N}$, 9846'E, 1.VIII.2005,

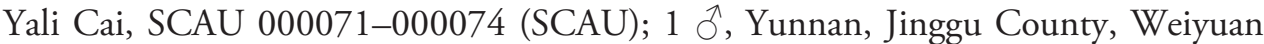
Town, $23^{\circ} 29^{\prime} \mathrm{N}, 100^{\circ} 42^{\prime} \mathrm{E}$, 4.X.2004, Jingxian Liu, SCAU 000075 (SCAU); 2 \%, Yunnan, Nabanhe Basin National Nature Reserve, $22^{\circ} 15.47^{\prime} \mathrm{N}, 100^{\circ} 36.32^{\prime} \mathrm{E}, 892 \mathrm{M}$, 
19-23.VII.2011, yellow pan trap, Zaifu Xu, SCAU000076, 000077 (SCAU). THAI-

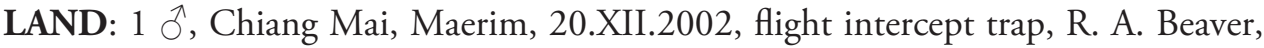
No. 25926 (RABC).

\section{Macroteleia carinigena sp. $\mathbf{n}$.}

urn:Isid:zoobank.org:act:42427976-EE7B-4B81-8910-EF308AE8716E

http://species-id.net/wiki/Macroteleia_carinigena

Plates 11-12

Description. Male. Body length 6.41-6.62 mm (n=3).

Color. Body black; mandible reddish brown; palpi yellow; legs yellow throughout; A1-A2 yellow, remainder of antenna brown to dark brown; fore wing hyaline.

Head. Transverse in dorsal view, 1.56-1.70× as wide as long, slightly wider than mesosoma; lateral ocellus contiguous with inner orbit of compound eye; POL 1.581.67x LOL; occipital carina continuous medially, irregularly crenulate throughout; central keel absent (Plate 12C); medial frons punctate with irregularly shaped smooth area; ventrolateral frons punctate rugulose to densely punctate; frons below median ocellus punctate reticulate; vertex densely punctate with punctures in part contiguous; gena with a strong carina parallel to occipital carina (Plate 12B), punctate rugose dorsally; length of A3 1.30-1.33× length of A2.

Mesosoma. Cervical pronotal area densely punctate; dorsal pronotal area areolate; lateral pronotal area smooth dorsally, irregularly depressed ventrally; netrion densely finely punctate; notaulus shallow, irregularly foveolate; middle lobe of mesoscutum densely punctate, becoming denser anteriorly; lateral lobes of mesoscutum densely punctate throughout; mesoscutellum densely punctate, becoming denser laterally; metascutellum transverse, posterior margin slightly pointed medially, longitudinally carinate (Plate 12A); propodeum continuous medially (Plate 12A), not divided into two separated lobes, posterior margin narrowly notched medially, each side with rugose sculpture covered by dense, recumbent, white setae; upper mesepisternum with a row of robust longitudinal carinae below subalar pit; lower mesepisternum densely punctate rugulose; mesopleural depression smooth (Plate 12B); metapleuron longitudinally striate with coarse punctures in interstices, or longitudinally punctate rugose.

Legs. Slender; hind femur weakly swollen, $4.00-4.55 \times$ as long as its maximum width; hind tibia without spines over outer surface; hind basitarsus $7.67-9.00 \times$ as long as its maximum width.

Wings. Apex of fore wing extending from as far as basal fifth to mid-length of T5; R 1.46-1.60× as long as r-rs, R1 1.95-2.43× length of R.

Metasoma. Posterior margin of transverse sulcus on T2 strongly convex (Plate 12A); sublateral tergal carinae well developed on T1-T3, weakly developed on anterior half of T4; T1-T3 sparsely longitudinally striate medially, with delicate punctures in interstices, punctate rugulose laterally; T4-T6 densely longitudinally striate, with numerous delicate punctures in interstices; T7 finely punctate rugulose posteriorly; T6 


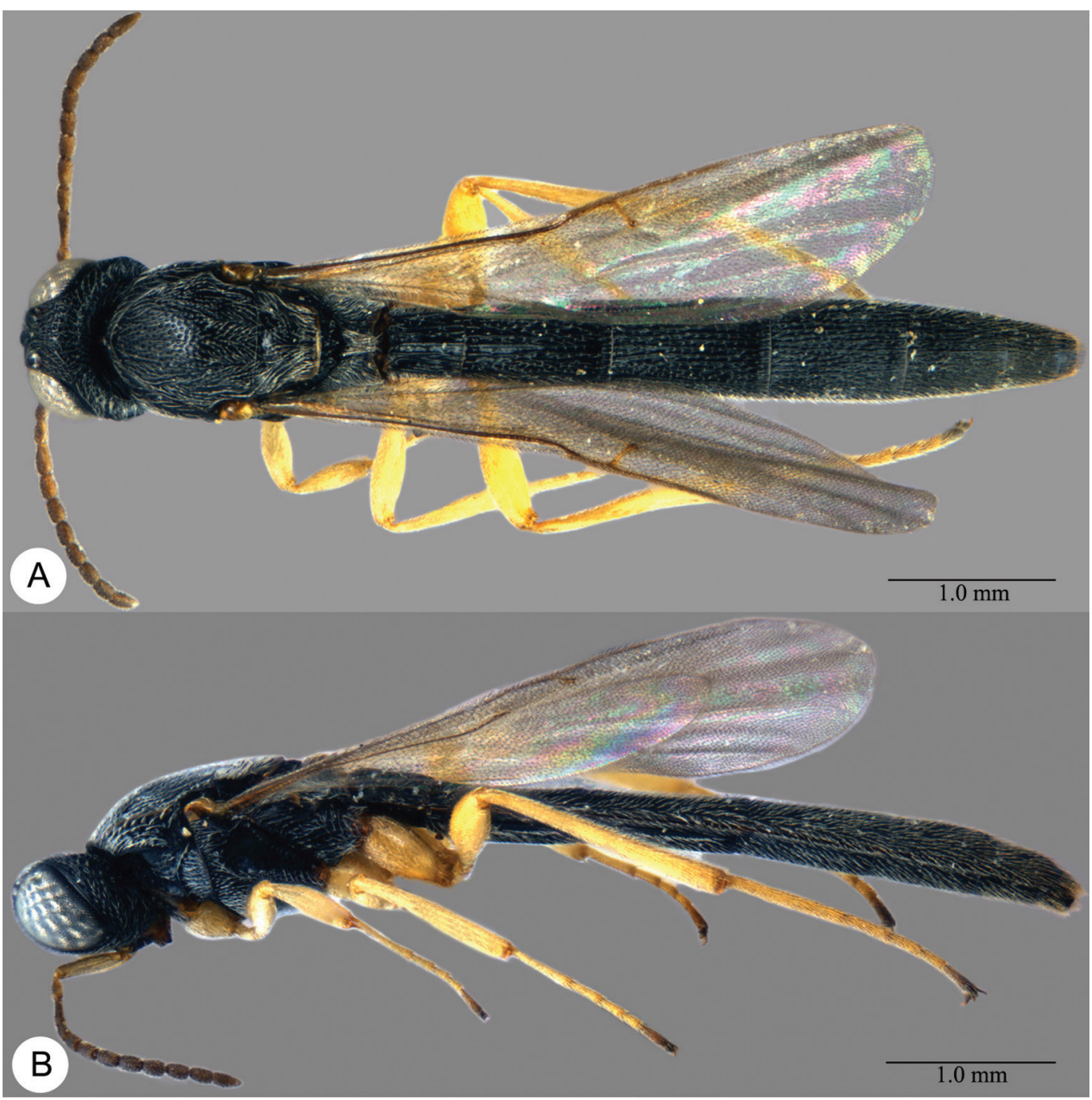

Plate I I. Macroteleia carinigena sp. n., holotype, male. A Dorsal habitus B Lateral habitus.

slightly wider than long; length of T6 2.35-2.63× length of T7; T7 transverse, apex truncate (Plate 12D); length of T7 equal to length of S7; S2-S3 sparsely longitudinally striate, with scattered delicate punctures and irregular microsculpture in interstices; S4-S6 densely longitudinally striate, with numerous delicate punctures in interstices; S7 densely punctate rugulose throughout; prominent longitudinal median carinae present on S2-S5.

Female. Unknown.

Diagnosis. This species is similar to $M$. boriviliensis, but can be distinguished by the presence of a strong carina on the gena (no carina in M. boriviliensis) and the propodeal lobe without longitudinal carinae (with several irregular longitudinal carinae medially in $M$. boriviliensis).

Etymology. The name carinigena refers to the strong carina present on gena of this species. The epithet is used as a noun in apposition. 


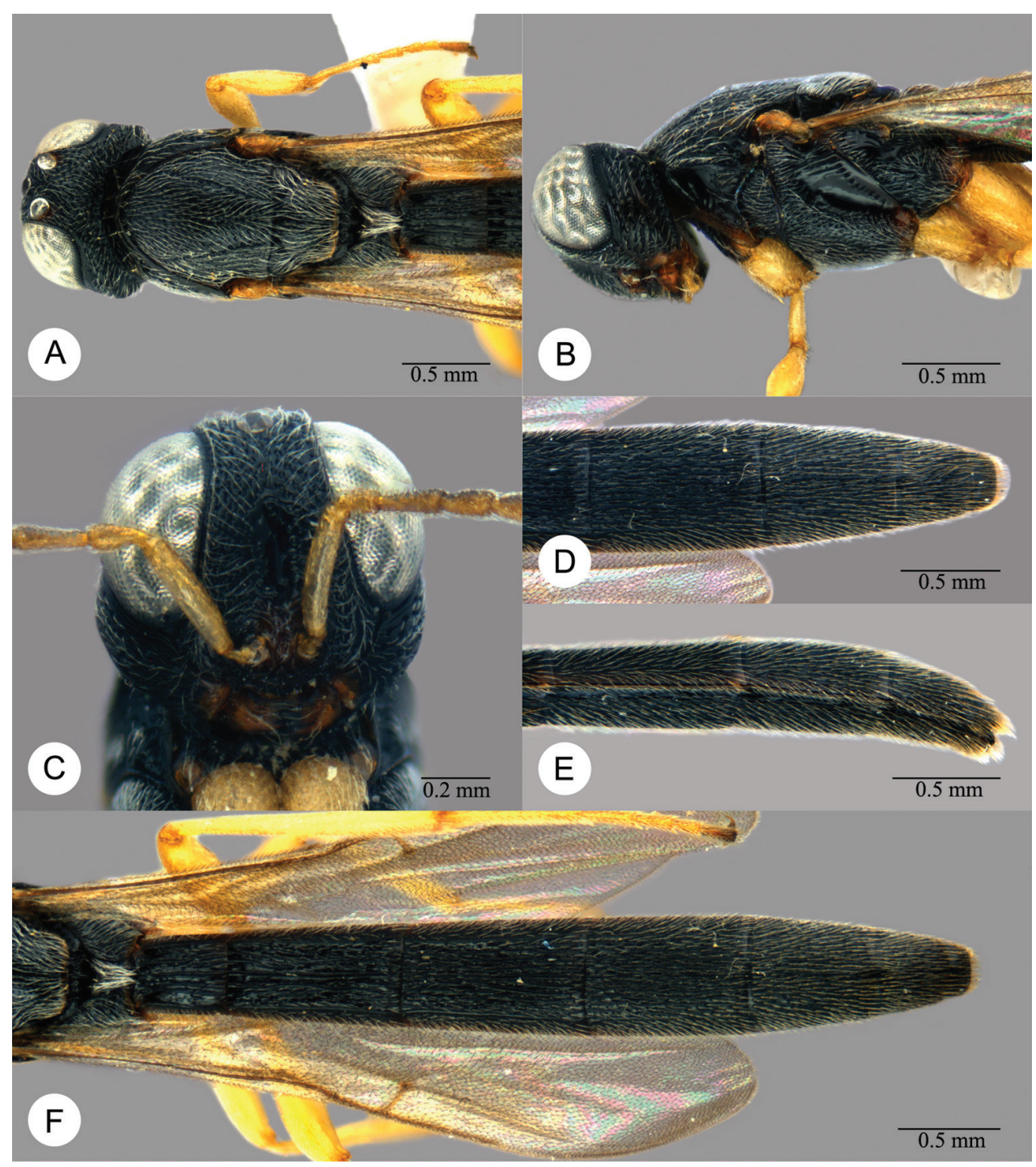

Plate I2. Macroteleia carinigena sp. n., holotype, male. A Head and mesosoma, dorsal view B Head and mesosoma, lateral view C Head, anterior view D Apex of metasoma, dorsal view E Apex of metasoma, lateral view $\mathbf{F}$ Metasoma, dorsal view.

Distribution. China (Hainan). Link to distribution map [http://hol.osu.edu/ map-large.html?id=320503].

Material examined. Holotype, $\widehat{O}^{\lambda}$ : CHINA: Hainan, Mt. Yinggeling, $18^{\circ} 49^{\prime} \mathrm{N}$, $109^{\circ} 11^{\prime} \mathrm{E}, 28 . V .2007$, Liqiong Weng, SCAU 000032 (deposited in SCAU). Paratypes:

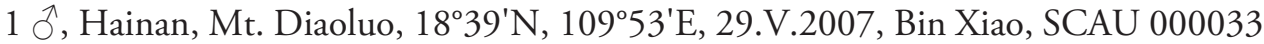
(SCAU); $1 \delta^{\lambda}$, Hainan, Mt. Diaoluo, $18^{\circ} 39^{\prime} \mathrm{N}, 109^{\circ} 53^{\prime} \mathrm{E}, 29 . V .2007$, Jingxian Liu, SCAU 000034 (SCAU). 


\section{Macroteleia crawfordi Kieffer, stat. n.}

Plates 13-17

Macroteleia kiefferi Crawford 1910: 127 (original description, preoccupied by Macroteleia kiefferi Brues (1906)); Baltazar 1966: 184 (listed, junior synonym of Macroteleia striativentris Crawford).

Macroteleia crawfordi Kieffer 1910b: 89 (replacement name for Macroteleia kiefferi Crawford); Baltazar 1966: 184 (listed, junior synonym of Macroteleia striativentris Crawford); Masner and Muesebeck 1968: 39 (type information). Macroteleia crates Lê 2000: 53, 55, 331 (original description, keyed), syn. n. Macroteleia demades Lê 2000: 53, 56, 332 (original description, keyed), syn. n.

Description. Female. Body length 3.35-4.76 mm ( $\mathrm{n}=20)$.

Color. Body black; mandible brown; palpi yellow; legs pale brown throughout; A1 brown, A2-A5 dark brown, remainder of antenna black; fore wing hyaline.

Head. Transverse in dorsal view, $1.30-1.40 \times$ as wide as long, slightly wider than mesosoma; OOL short, $0.20-0.40 \times$ times minimum diameter of lateral ocellus; POL 1.27-1.58× LOL; occipital carina continuous medially, irregularly crenulate; central keel weakly developed above interantennal process (Plates 14A, 16C); medial frons obliquely strigose ventrally, variably smooth to coriaceous dorsally; ventrolateral frons punctate rugose; frons below median ocellus densely punctate, interspaces coriaceous; ocellar triangle smooth or coriaceous, with scattered punctures; posterior vertex densely punctate, interspaces coriaceous; gena coarsely punctate rugose; length of A3 0.71 $0.86 \times$ length of A2.

Mesosoma. Cervical pronotal area densely punctate; dorsal pronotal area areolate; lateral pronotal area punctate rugulose; netrion densely punctate or punctate rugulose; notaulus deep, distinctly foveolate; middle lobe of mesoscutum densely punctate, becoming denser anteriorly; lateral lobes of mesoscutum densely punctate throughout; mesoscutellum densely punctate throughout; metascutellum tongue-like (Plates 14B, $16 \mathrm{D}, 17 \mathrm{D})$, extending into space between propodeal lobes; propodeum narrowly divided into two widely separated subtriangular lobes (Plates 14B, 16D, 17D), each side with punctate rugulose sculpture covered by dense, recumbent, white setae; upper mesepisternum with a row of robust longitudinal carinae below subalar pit; lower mesepisternum densely punctate; mesopleural depression smooth (Plates 14C, 16E, $17 \mathrm{E})$; metapleuron longitudinally striate dorsally, densely punctate ventrally.

Legs. Slender; hind femur weakly swollen, $4.06-4.71 \times$ as long as its maximum width; hind tibia without spines over outer surface; hind basitarsus $11.00-11.75 \times$ as long as its maximum width.

Wings. Apex of fore wing extending from as far as posterior margin of $\mathrm{T} 4$ to base of T5; R 1.79-2.55× as long as r-rs, R1 1.18-1.77× length of R.

Metasoma. Posterior margin of transverse sulcus on T2 straight or slightly convex (Plate 14D); sublateral tergal carinae developed on T1-T4; T1 densely longitudinally striate medially, with numerous punctures in interstices anteriorly, punctate rugu- 


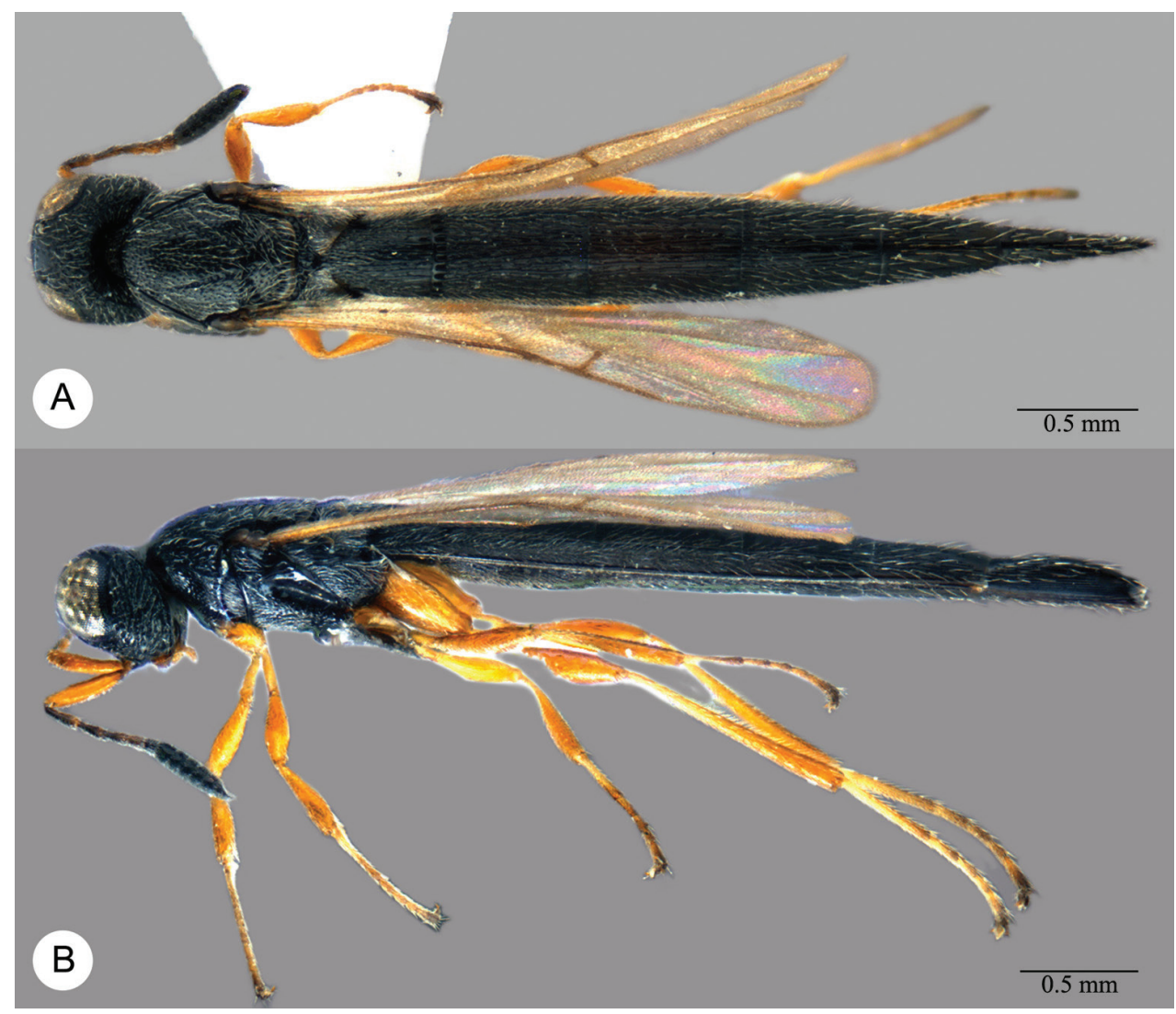

Plate 13. Macroteleia crawfordi Kieffer, female from Guangdong, Chebaling National Nature Reserve. A Dorsal habitus B Lateral habitus.

lose laterally; T2-T4 densely longitudinally striate medially, with scattered delicate punctures in interstices, punctate rugulose laterally; T5 densely longitudinally striate throughout, with delicate punctures in interstices; T6 finely punctate dorsally, densely longitudinally striate laterally, with scattered delicate punctures in interstices; length of T3 $0.82-0.99 \times$ length of T6; T5 distinctly longer than wide; S2-S4 densely longitudinally striate, with delicate punctures in interstices; S5-S6 largely smooth, with scattered punctures; prominent longitudinal median carina present on S2-S5.

Male. Differing from female as follows: body length 3.57-3.85 mm ( $\mathrm{n}=6) ; \mathrm{A} 1$ brown, remainder of antenna dark brown to black; metascutellum distinctly transverse (Plate 15A), posterior margin straight, longitudinally carinate; propodeum continuous medially (Plate 15A), not divided into two separated lobes, posterior margin narrowly notched medially, each side with several irregular longitudinal carinae medially, otherwise punctate rugulose, covered by dense, recumbent, white setae; T1-T4 denselylongitudinally striate medially, with delicate punctures in interstices, punctate rugulose laterally; T5-T7 densely longitudinally striate throughout, with delicate punctures 


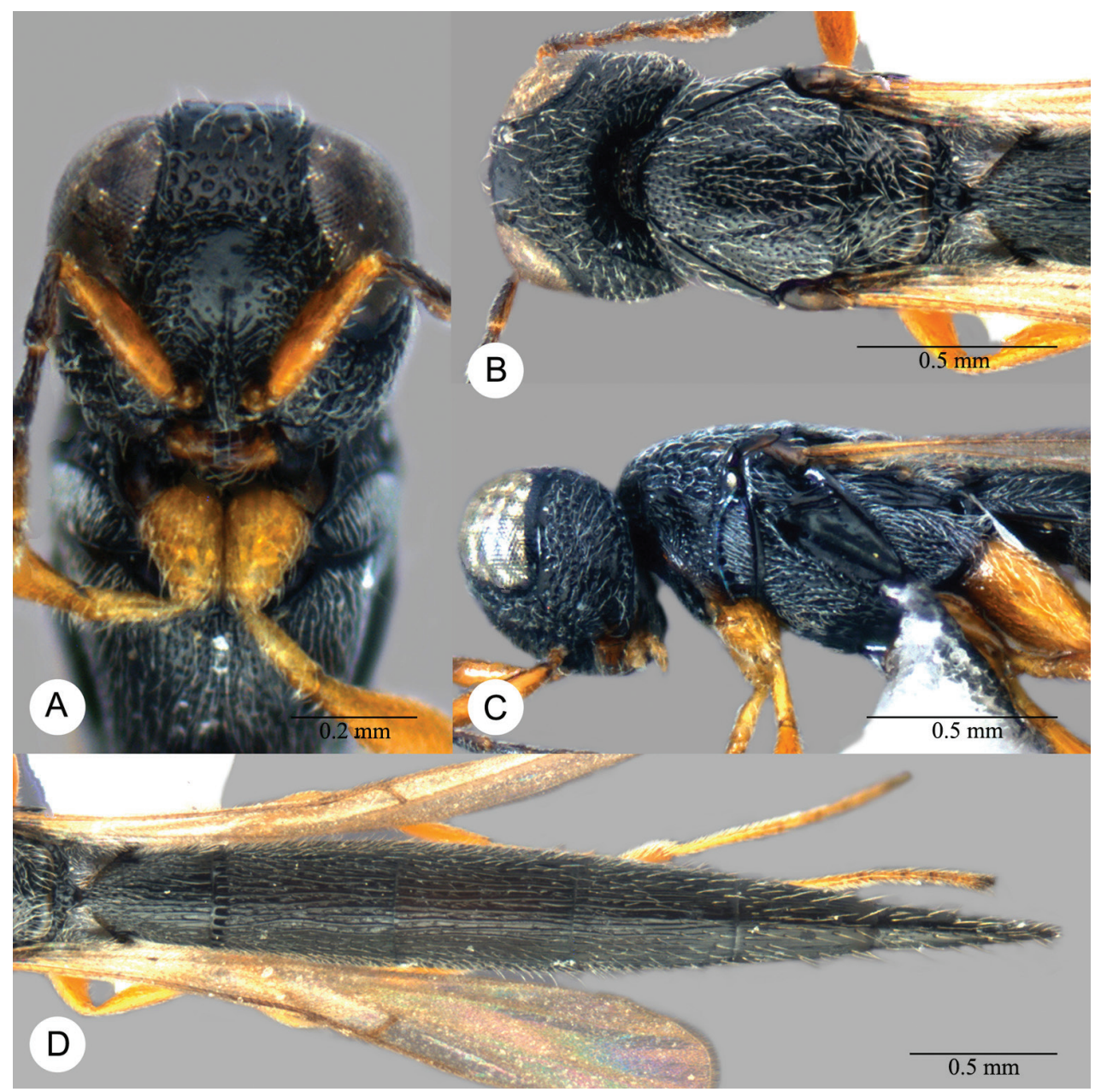

Plate 14. Macroteleia crawfordi Kieffer, female from Guangdong, Chebaling National Nature Reserve. A Head, anterior view B Head and mesosoma, dorsal view C Head and mesosoma, lateral view D Metasoma, dorsal view.

in interstices; T6 slightly wider than long; length of T6 1.00-1.13× length of T7; T7 subtriangular, apex sharply pointed to form a spine (Plate 15B, C); length of T7 1.13-1.77x length of S7; S2-S3 densely longitudinally striate, with punctate rugulose sculpture in interstices; S4-S6 densely longitudinally striate, with delicate punctures in interstices; $\mathrm{S} 7$ longitudinally punctate rugulose; prominent longitudinal median carina present on S2-S6.

Diagnosis. The body shape, color and size of M. crawfordi is similar to M. lamba and $M$. livingstoni. It differs from them by tongue-like metascutellum in female (triangular in $M$. lamba, and transverse in $M$. linvingstoni), and apex of T7 sharply pointed to form a spine in male (apex not pointed to form a spine in the latter two speices). 


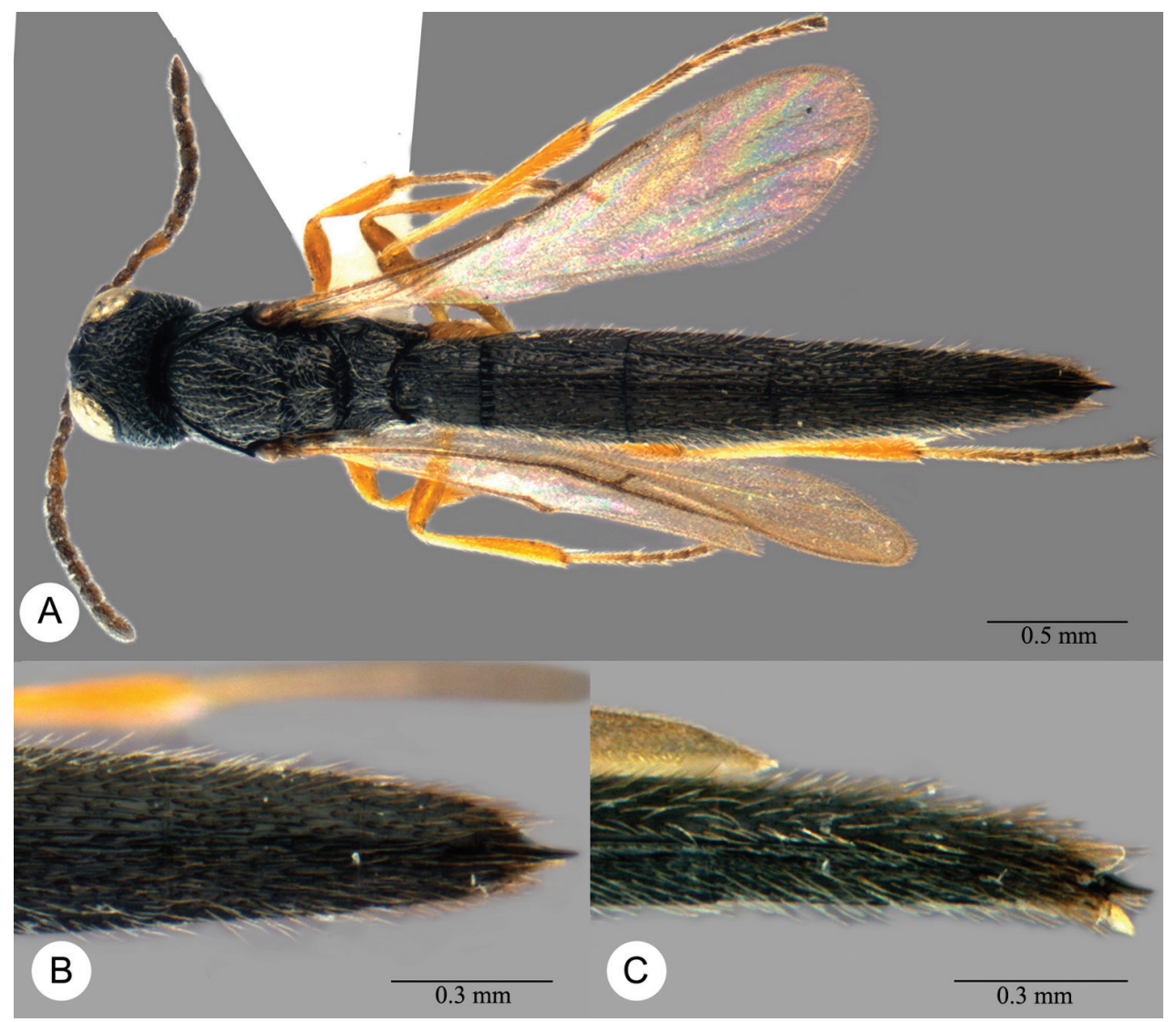

Plate 15. Macroteleia crawfordi Kieffer, male from Guangdong, Chebaling National Nature Reserve. A Dorsal habitus B Apex of metasoma, dorsal view C Apex of metasoma, lateral view.

Distribution. China (Guangdong, Hainan); Vietnam; Thailand; Philippines. Link to distribution map [http://hol.osu.edu/map-large.html?id=9295].

Material examined. Holotype of M. crawfordi, o : PHILIPPINES: "Philippines, Manila, R. Brown, USNM No.12897" (deposited in USNM). Holotype of M. crates, , VIETNAM: "8/4 construction site (Dak Lak Prov.), rice field, 15.V.1979", "Holotypus + Macroteleia crates Kozlov et Lê 84". Holotype of M. demades, + (deposited in IEBR): VIETNAM: "Nghia Do, Hanoi, on rice seedling, 24.VII.1978", "Holotypus + Macroteleia demades Kozlov et Lê 84" (deposited in IEBR). Paratypes of M. demades, 3 q (IEBR): 2 +, VIETNAM: "Nghia Do, Hanoi, on rice seedling, 24.VII.1978, Lê Xuan Hue", "Paratypus Macroteleia demades sp. n."; 1 + , VIETNAM: "Ha Noi, rice field, 15.VII.1978", "Paratypus Macroteleia demades sp. n.".

Other material. CHINA: 1 , Guangdong, Chebaling National Nature Reserve, $24^{\circ} 43^{\prime} \mathrm{N}, 114^{\circ} 14^{\prime} \mathrm{E}, 25 . \mathrm{V} .2002$, Jingxian Liu, SCAU 000139 (SCAU); $10+9+1$ ô, Guangdong, Chebaling National Nature Reserve, $24^{\circ} 43^{\prime} \mathrm{N}, 114^{\circ} 14^{\prime} \mathrm{E}, 21-23$. 


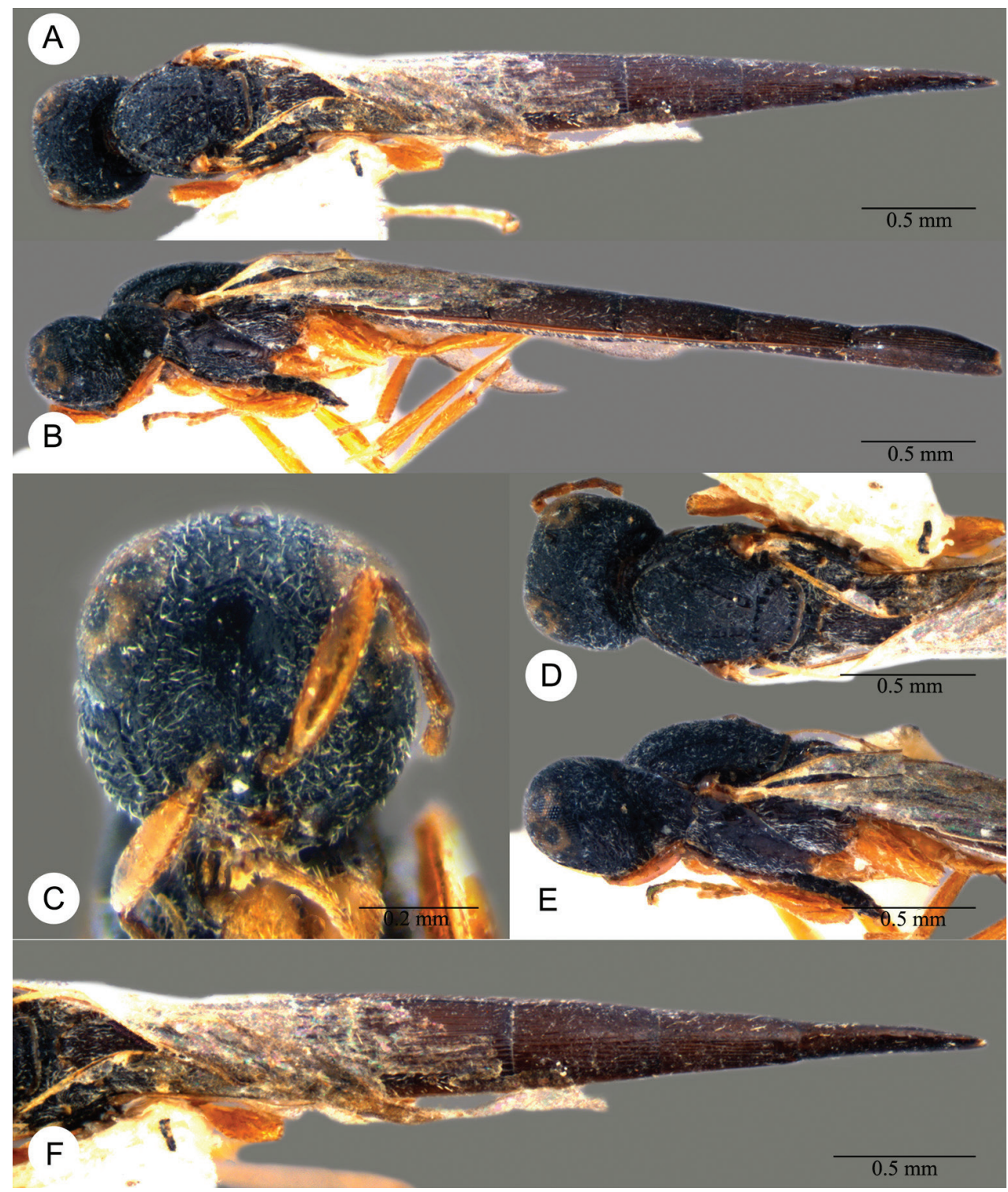

Plate 16. Macroteleia crates Kozlov \& Lê, holotype, female. A Dorsal habitus B Lateral habitus C Head, anterior view $\mathbf{D}$ Head and mesosoma, dorsal view $\mathbf{E}$ Head and mesosoma, lateral view $\mathbf{F}$ Metasoma, dorsal view.

VIII.2003, Jingxian Liu, SCAU 000140-000150 (SCAU); 1 + +1 $\lesssim$, Guangdong, Zijin County, Linjiang Town, $23^{\circ} 39^{\prime} \mathrm{N}, 114^{\circ} 41^{\prime} \mathrm{E}, 1$.VIII.2003, Jingxian Liu, SCAU

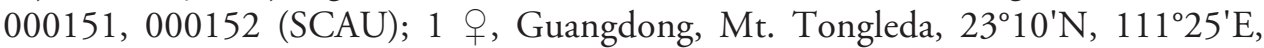
12.VIII.2003, Jujian Chen, SCAU 000153 (SCAU); 1 क , Guangdong, Zhuhai, Doumen, paddy field, $22^{\circ} 33.366^{\prime} \mathrm{N}, 113^{\circ} 13.297^{\prime} \mathrm{E}, 11 . \mathrm{IX} .2010$, Xin Yuan, SCAU

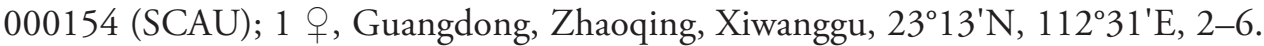




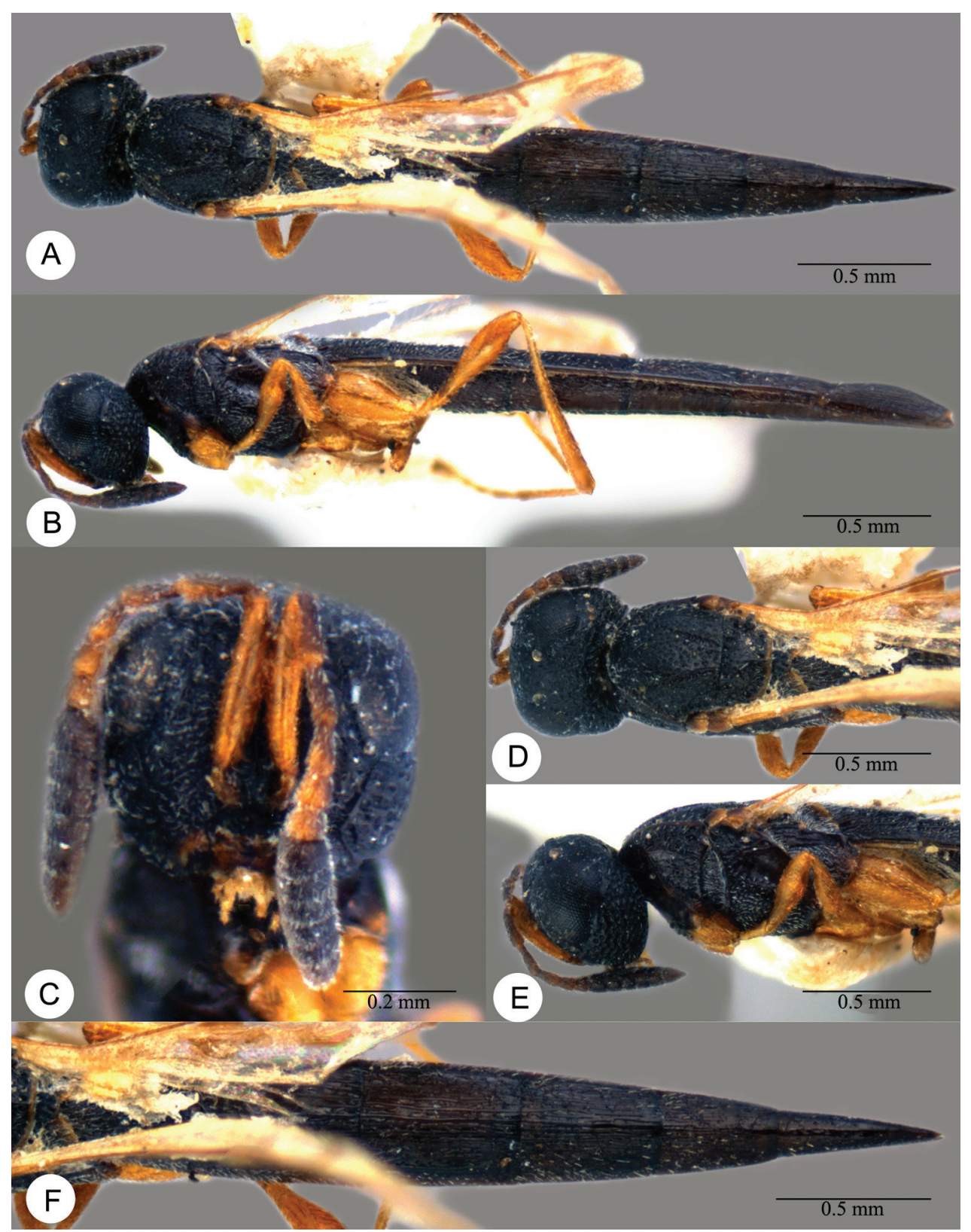

Plate 17. Macroteleia demades Kozlov \& Lê, holotype, female. A Dorsal habitus B Lateral habitus C Head, anterior view D Head and mesosoma, dorsal view E Head and mesosoma, lateral view F Metasoma, dorsal view.

VIII.2010, yellow pan trap, Huayan Chen, SCAU 000155 (SCAU); 2 \%, Hainan, Wuzhishan National Nature Reserve, $18^{\circ} 51^{\prime} \mathrm{N}, 109^{\circ} 39^{\prime} \mathrm{E}, 16-18 . V .2007$, Jie Zeng, SCAU 000156, 000157 (SCAU); 1 , , Hainan, Wuzhishan National Nature Reserve, 
$18^{\circ} 51^{\prime} \mathrm{N}, 109^{\circ} 39^{\prime} \mathrm{E}, 17-20 . V .2007$, Bin Xiao, SCAU 000158 (SCAU); 1 ㅇ, Hainan, Wuzhishan National Nature Reserve, $18^{\circ} 51^{\prime} \mathrm{N}, 109^{\circ} 39^{\prime} \mathrm{E}, 20 . \mathrm{V} .2007$, Jingxian

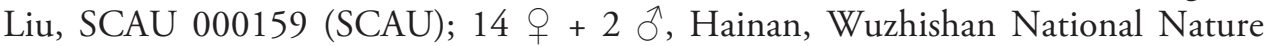
Reserve, $18^{\circ} 51^{\prime} \mathrm{N}, 109^{\circ} 39^{\prime} \mathrm{E}, 28 . X-30 . X .2007$, Jiemin Yao, SCAU 000160-000175 (SCAU); 7 9, Hainan, Wuzhishan National Nature Reserve, $18^{\circ} 51^{\prime} \mathrm{N}, 109^{\circ} 39^{\prime} \mathrm{E}$, 29.X.2007, Jingxian Liu, SCAU 000176-000182 (SCAU); 2 + , Hainan, Jianfengling National Nature Reserve, $18^{\circ} 41^{\prime} \mathrm{N}, 108^{\circ} 49^{\prime} \mathrm{E}, 23-25 . X .2007$, Jiemin Yao, SCAU 000183, 000184 (SCAU); 1 \%, Hainan, Mt. Yinggeling, $18^{\circ} 49^{\prime} \mathrm{N} 109^{\circ} 11^{\prime} \mathrm{E}$, 16-20.XI.2008, Huayan Chen, SCAU 000185 (SCAU); 2 + 1 ภे, Hainan, Mt. Yinggeling, $18^{\circ} 49^{\prime} \mathrm{N}, 109^{\circ} 11^{\prime} \mathrm{E}, 17-20 . V I I .2010$, Huayan Chen, SCAU $000186-$ 000188 (SCAU). THAILAND: 1 \%, Chiang Mai: Maerim, 29-30.XII.2000, MT, R. A. Beaver, No. 24139 (RABC); 1 \%, Chiang Mai: Maerim, 24-25.I.2001, MT, R. A. Beaver, No. 24671 (RABC).

\section{Macroteleia dolichopa Sharma}

urn:lsid:zoobank.org:act:BF0454A0-EF33-4A82-BC87-33ACDF973231

http://species-id.net/wiki/Macroteleia_dolichopa

Plates 18-21

Macroteleia dolichopa Sharma 1980: 300 (original description); Mani and Sharma 1982: 171 (description).

Description. Female. Body length 5.13-7.50 mm (n=8).

Color. Head black; mesosoma orange yellow; metasoma with T1, T5 and T6 variably dark brown to black, otherwise orange yellow; mandible dark brown; palpi yellow; legs yellow throughout; A1-A6 yellowish brown, A7-A12 dark brown to black; fore wing hyaline.

Head. Transverse in dorsal view, $1.42-1.44 \times$ as wide as long, slightly wider than mesosoma; lateral ocellus contiguous with inner orbit of compound eye; POL 1.361.67x LOL; occipital carina continuous medially, irregularly punctate; central keel absent (Plates 18C, 20C); medial frons punctate rugulose ventrally, irregularly smooth dorsally; ventrolateral frons punctate rugose; frons below median ocellus punctate reticulate; ocellar triangle largely smooth, with scattered punctures; posterior vertex punctate reticulate; gena punctate rugose; length of A3 equal to length of A2.

Mesosoma. Cervical pronotal area densely punctate; dorsal pronotal area punctate rugose; lateral pronotal area smooth anteriorly, punctate rugulose posteriorly; netrion punctate rugulose; notaulus contiguously foveolate; middle lobe of mesoscutum densely punctate, becoming denser anteriorly and at posterior end; lateral lobes of mesoscutum densely punctate throughout; mesoscutellum finely punctate throughout; metascutellum transverse (Plates 18D, 20B), posterior margin slightly pointed medially, longitudinally carinate; propodeum continuous medially (Plates 18D, 20B), not divided into two separated lobes, posterior margin narrowly notched medially, each side with several irregular longitudinal carinae medially, otherwise punctate ru- 


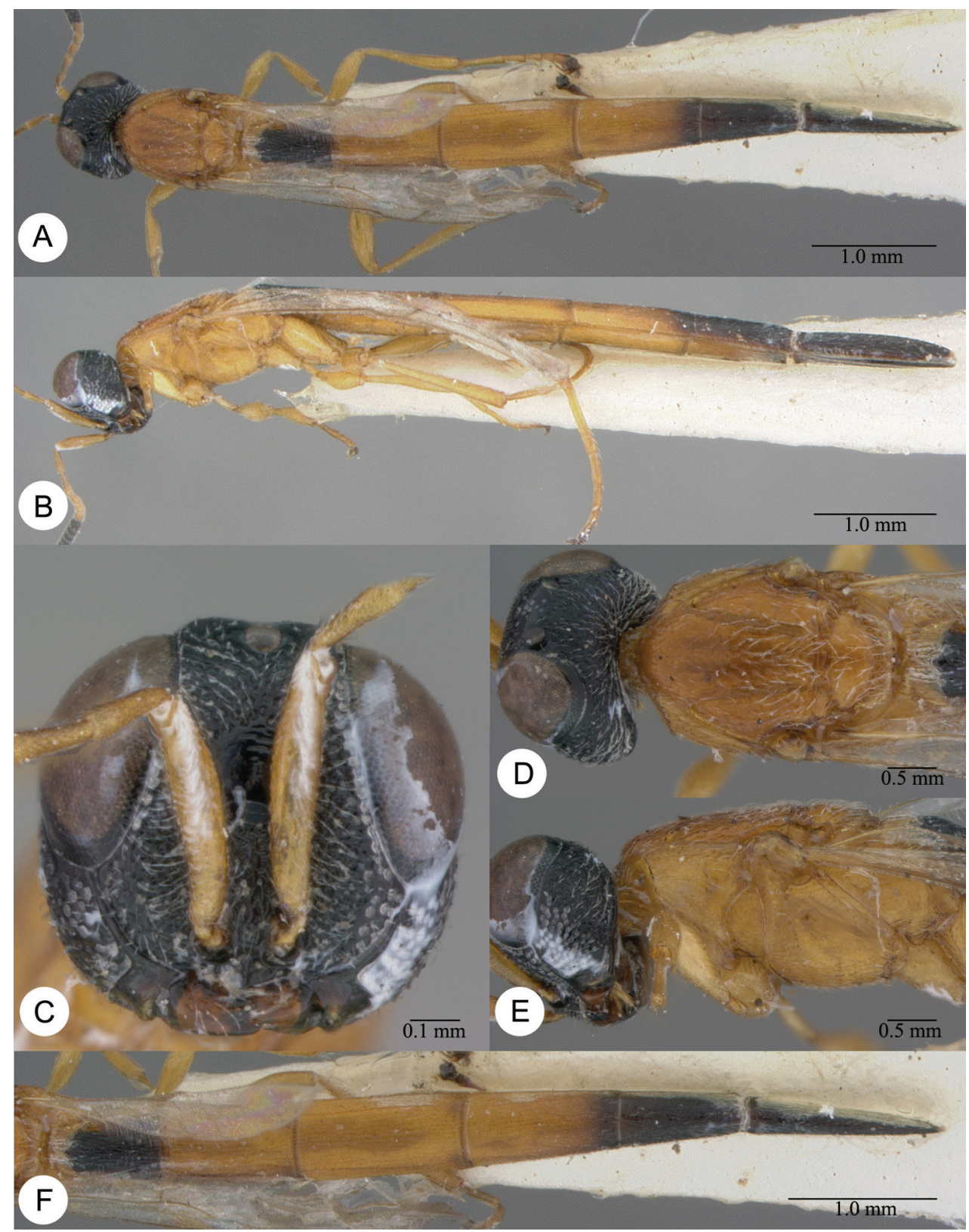

Plate 18. Macroteleia dolichopa Sharma, holotype, female. A Dorsal habitus B Lateral habitus C Head, anterior view D Head and mesosoma, dorsal view E Head and mesosoma, lateral view F Metasoma, dorsal view.

gulose, covered by dense, recumbent, white setae; upper mesepisternum with a row of fine longitudinal carinae below subalar pit; lower mesepisternum punctate rugulose; mesopleural depression smooth (Plates 18E, 20C); metapleuron longitudinally striate dorsally, punctate rugulose ventrally. 


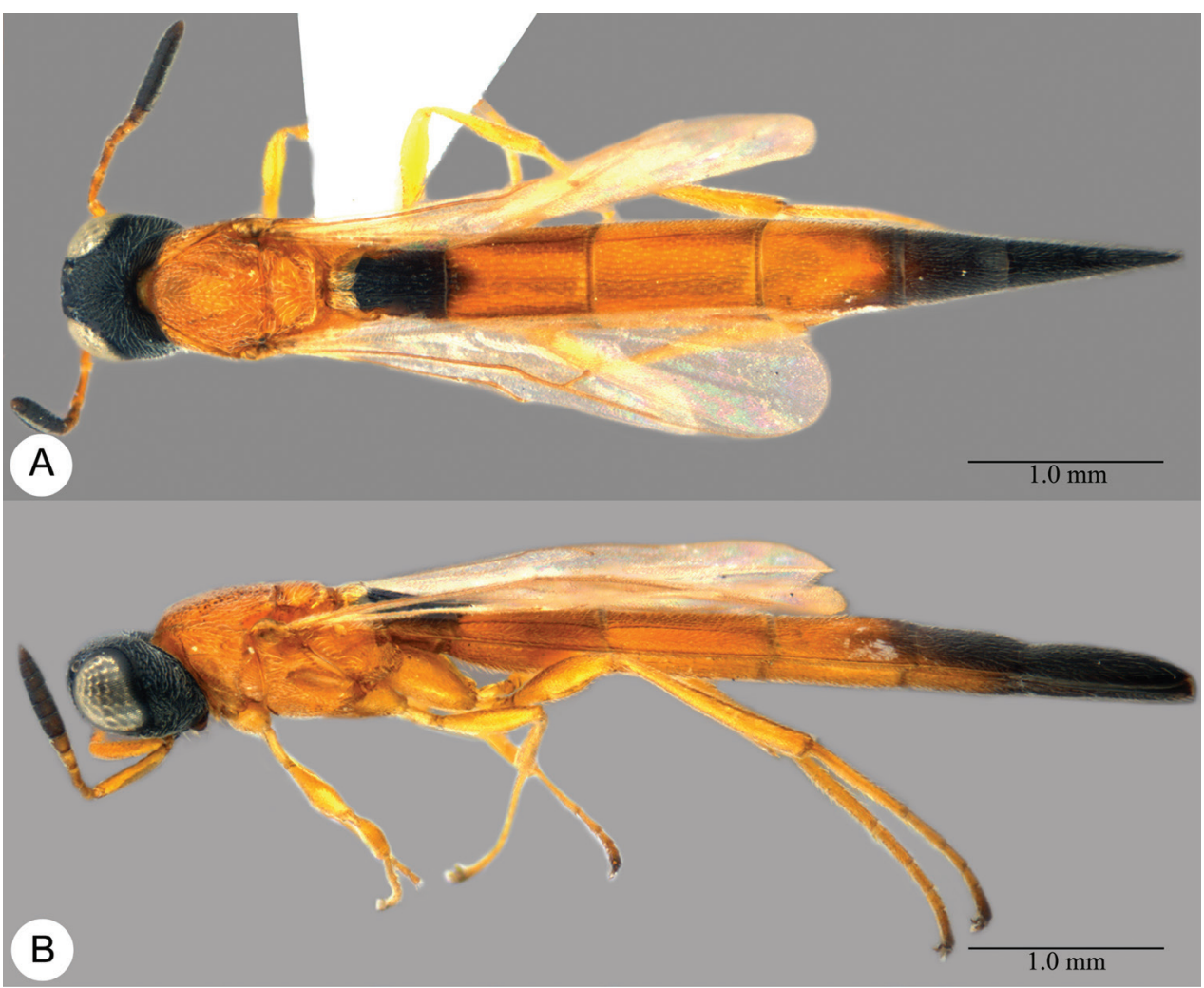

Plate 19. Macroteleia dolichopa Sharma, female from Guangdong, Xinfeng, Mt. Yunji. A Dorsal habitus B Lateral habitus.

Legs. Slender; hind femur weakly swollen, $3.85-4.38 \times$ as long as its maximum width; hind tibia without spines over outer surface; hind basitarsus 9.50-12.33× as long as its maximum width.

Wings. Apex of fore wing extending as far as mid-length of T4 to mid-length of T5; R 1.47-2.41× as long as r-rs, R1 1.57-2.40× length of R.

Metasoma. Posterior margin of transverse sulcus on T2 slightly convex (Plate 20D); sublateral tergal carinae developed on T1-T3; T1 densely longitudinally striate medially, with scattered punctures in interstices anteriorly, rugulose laterally; T2-T3 densely longitudinally striate medially, with delicate punctures in interstices, punctate rugulose laterally; T4-T5 densely finely longitudinally striate throughout, with delicate punctures in interstices; T6 finely punctate dorsally, densely longitudinally striate laterally, with scattered small punctures in interstices; length of T3 $0.95-1.15 \times$ length of T6; T5 slightly longer than wide; S2-S4 longitudinally striate, with finely punctate rugulose interstices; S5-S6 longitudinally striate, with finely punctate interstices; prominent longitudinal median carina present on S2-S5. 


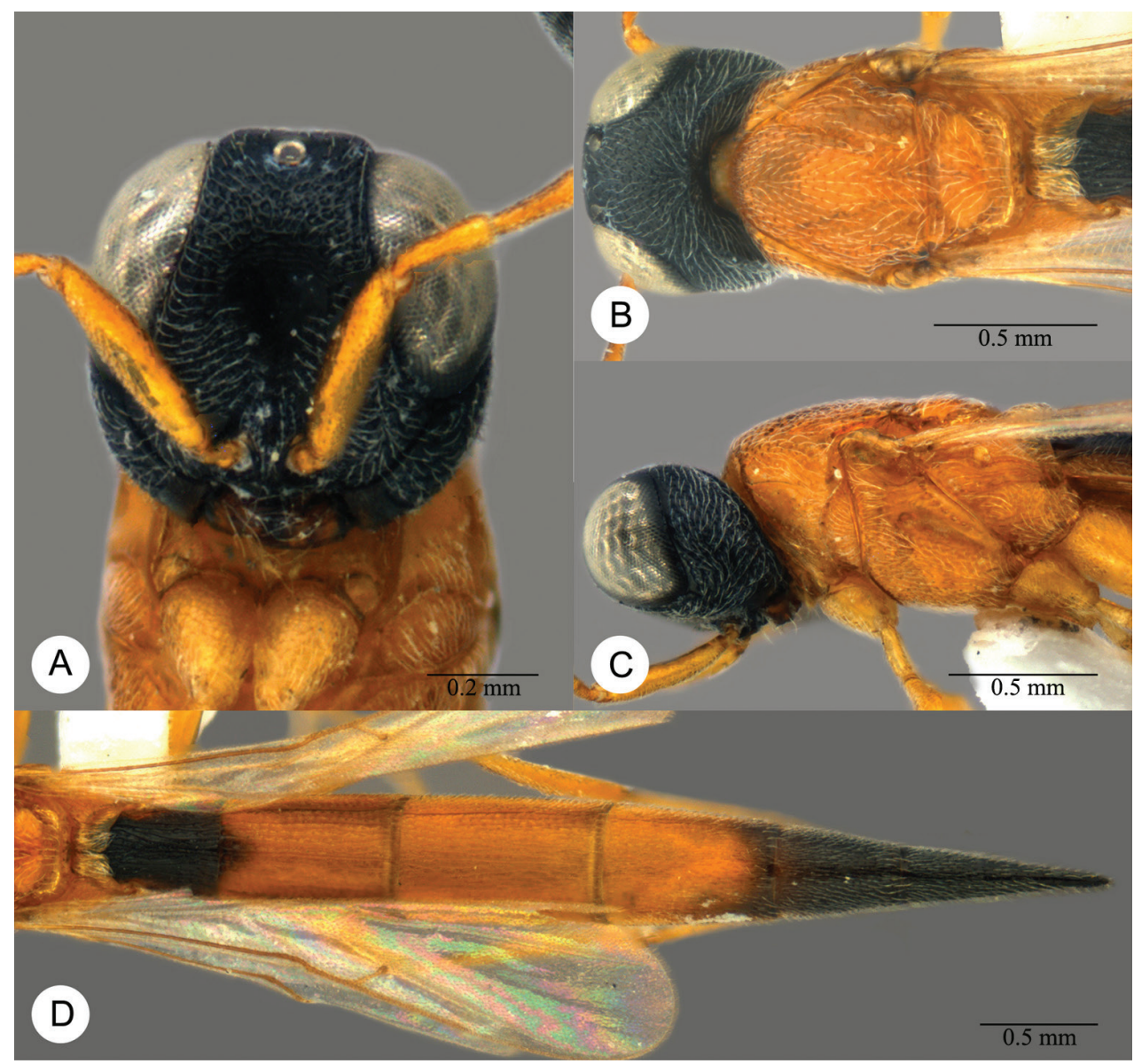

Plate 20. Macroteleia dolichopa Sharma, female from Guangdong, Xinfeng, Mt. Yunji. A Head, anterior view B Head and mesosoma, dorsal view C Head and mesosoma, lateral view D Metasoma, dorsal view.

Male. Differing from female as follows: body length 5.21-5.41 mm ( $\mathrm{n}=5)$; mesosoma orange yellow or brown; metasoma variably brown to black; A1 yellow, remainder of antenna brown to dark brown; T1 sparsely longitudinally striate medially, with punctate rugulose interstices anteriorly, punctate rugulose laterally; T6-T7 longitudinally punctate rugulose; T6 slightly longer than wide; length of T6 1.10-1.33× length of T7; T7 subtriangular, apex sharply pointed (Plate 21B, C); length of T7 1.55-1.80× length of S7; S6-S7 longitudinally punctate rugulose.

Diagnosis. The male of this species is similar to that of $M$. striativentris in body size, but can be separated by the combination of the black head; T7 sharply pointed medially; metapleuron longitudinally striate dorsally, punctate rugulose ventrally.

Distribution. China (Hubei, Guangdong); Vietnam; India. Link to distribution map [http://hol.osu.edu/map-large.html?id=4798]. 


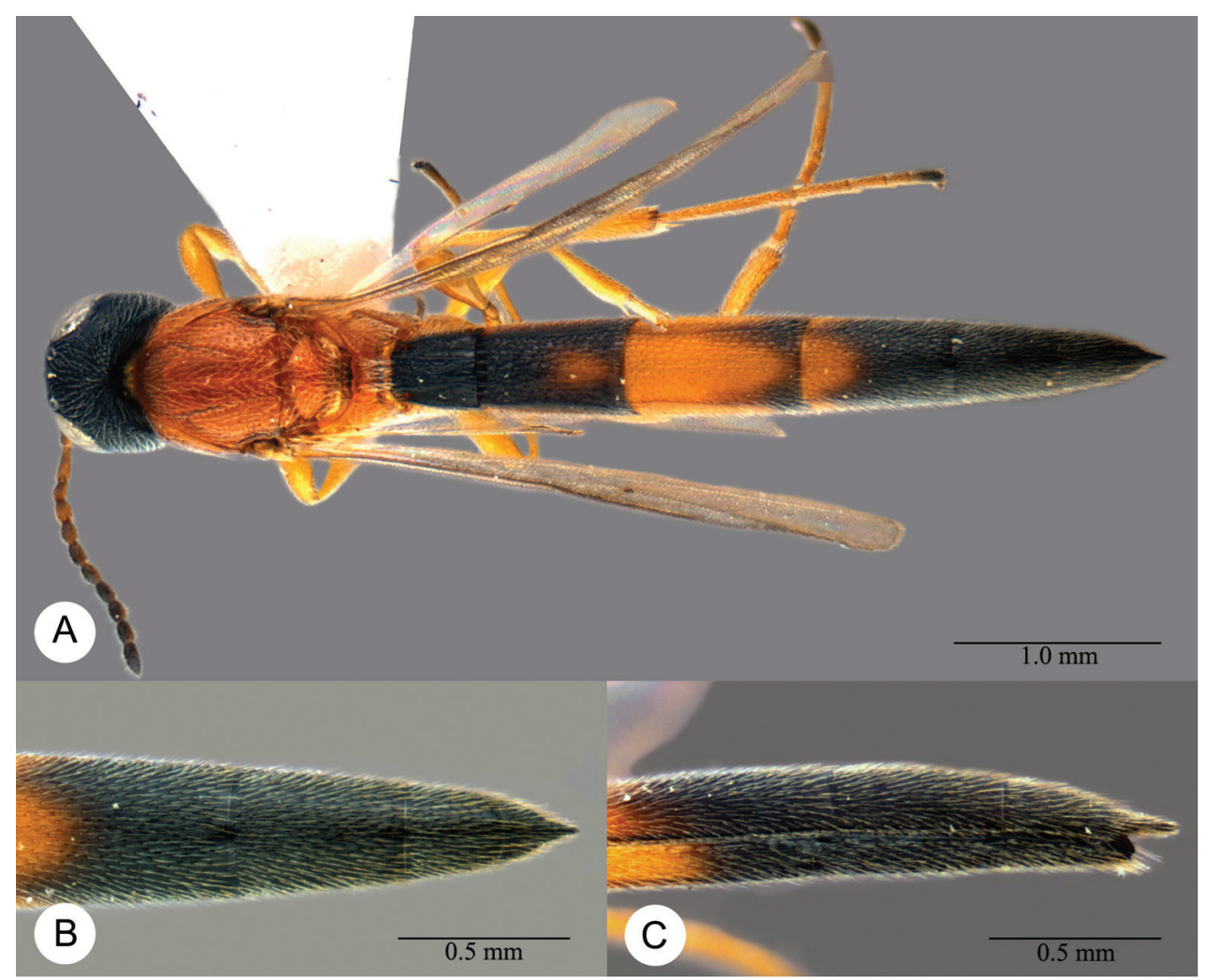

Plate 2I. Macroteleia dolichopa Sharma, male from Guangdong, Mt. Nankun. A Dorsal habitus B Apex of metasoma, dorsal view C Apex of metasoma, lateral view.

Material examined. Holotype, ô: INDIA: "Vindhya Survey, School of Entomology, St. John's College, Agra-282002, India, 21.5. Harsa, M.S. Mani \& Party, 9-10. IX.1979", "Holotype", "Macroteleia dolichopa Sharma, S.K., @” (deposited in USNM).

Other material. CHINA: 1 , Guangdong, Nanling National Nature Reserve, $24^{\circ} 54^{\prime} \mathrm{N}, 113^{\circ} 00^{\prime} \mathrm{E}, 9-18 . \mathrm{VII} .2004$, Juanjuan Ma, SCAU 000035 (SCAU); 1 , Hubei, Huanggang, Mt. Dahu, $31^{\circ} 27^{\prime} \mathrm{N}, 14^{\circ} 32^{\prime} \mathrm{E}$, VIII.2009, Chunhong Zheng, SCAU 0000502 (SCAU); 1\$, Guangdong, Chebaling National Nature Reserve, $24^{\circ} 43^{\prime} \mathrm{N}, 14^{\circ} 14^{\prime} \mathrm{E}, 23-28 . V I I .2008$, Zaifu Xu, SCAU 000038 (SCAU); 1 \% , Guangdong, Nanling National Nature Reserve, $24^{\circ} 54^{\prime} \mathrm{N}, 113^{\circ} 00^{\prime} \mathrm{E}$, 8-17.VIII.2010, sweeping, Huayan Chen, SCAU 000037 (SCAU); 1 \%, Guangdong, Nanling National Nature Reserve, $24^{\circ} 54^{\prime} \mathrm{N}, 113^{\circ} 00^{\prime} \mathrm{E}$, 16-21.VII.2008, Zaifu Xu, SCAU 000036 (SCAU);

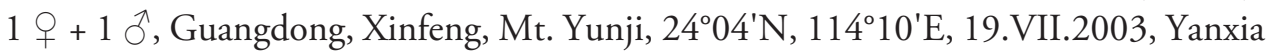

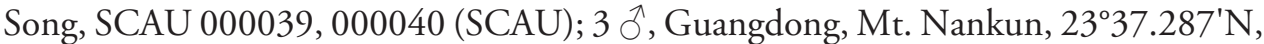
$113^{\circ}$ 51.267'E, 2.VII.2005, Zaifu Xu, SCAU 000041-000043 (SCAU). VIETNAM: 1 ô, "Bac Thai, Phu Luong, Quan Chu, 21.IV.1986, A. Sarkov", "Paratypus, Macroteleia fugacious sp. n.” (IEBR). 


\section{Macroteleia emarginata Dodd}

urn:lsid:zoobank.org:act:76F8C774-CBF3-49EA-BEB5-63E02F148EFF

http://species-id.net/wiki/Macroteleia_emarginata

Plates 22-24

Macroteleia emarginata Dodd 1920: 326 (original description); Masner, 1965: 82 (type information).

Description. Female. Body length 5.48-6.14 mm ( $\mathrm{n}=18)$.

Color. Body black; mandible reddish brown; palpi brown; base of hind coxa blackish, remainder of legs pale brown, becoming darker distally; A1 brown, A2-A5 dark brown, remainder of antenna black; fore wing hyaline.

Head. Transverse in dorsal view, $1.30-1.50 \times$ as wide as long, slightly wider than mesosoma; OOL short, $0.10-0.31 \times$ times minimum diameter of lateral ocellus; POL 1.29-1.64× LOL; occipital carina continuous medially, irregularly punctate; central keel absent (Plate 23A); medial frons obliquely strigose ventrally, irregularly smooth dorsally; ventrolateral frons punctate rugose; frons below median ocellus densely punctate, with punctures in part contiguous; ocellar triangle largely smooth, with scattered punctures; posterior vertex densely punctate, with punctures in part contiguous; gena coarsely punctate rugose; length of A3 equal to length of A2.

Mesosoma. Cervical pronotal area densely punctate; dorsal pronotal area densely coarsely punctate; lateral pronotal area smooth anteriorly, punctate rugulose ventrally; netrion densely finely punctate; notaulus deep, distinctly foveolate; middle lobe of mesoscutum densely punctate anteriorly and at posterior end, usually sparsely punctate on a small area across middle; lateral lobe of mesoscutum densely punctate throughout; mesoscutellum densely punctate throughout; metascutellum transverse (Plate 23B), posterior margin slightly pointed medially, longitudinally carinate; propodeum continuous medially (Plate 23B), not divided into two separated lobes, posterior margin narrowly notched medially, each side with several irregular longitudinal carinae medially, otherwise punctate rugulose, covered by dense, recumbent, white setae; upper mesepisternum with a row of robust longitudinal carinae below subalar pit; lower mesepisternum variably smooth to punctate rugulose; mesopleural depression smooth (Plate 23C); metapleuron longitudinally striate dorsally, punctate rugulose ventrally.

Legs. Slender; hind femur weakly swollen, $3.68-4.76 \times$ as long as its maximum width; hind tibia without spines over outer surface; hind basitarsus $9.44-12.33 \times$ as long as its maximum width.

Wings. Apex of fore wing extending from as far as posterior fourth of T4 to anterior third of T5; R 1.41-1.94× as long as r-rs, R1 1.73-2.26× length of R.

Metasoma. Posterior margin of transverse sulcus on T2 slightly to strongly convex (Plate 23D); sublateral tergal carinae developed on T1-T3; T1 densely longitudinally striate, with punctate rugulose sculpture in interstices anteriorly, punctate rugulose laterally; T2-T3 densely longitudinally striate medially, with delicate punctures in in- 


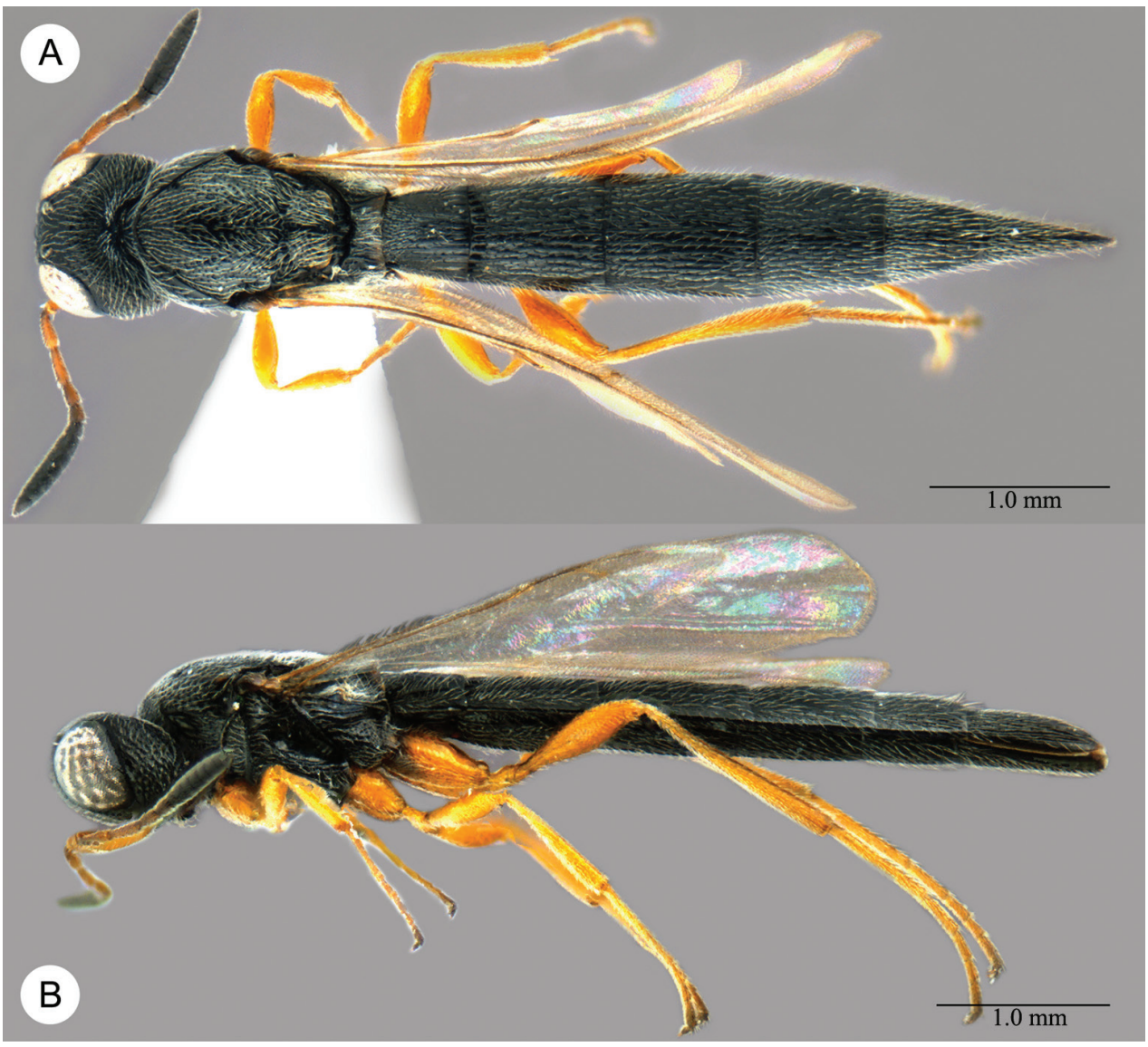

Plate 22. Macroteleia emarginata Dodd, female from Guangdong, Mt. Nankun. A Dorsal habitus B Lateral habitus.

terstices, punctate rugulose laterally; T4-T5 densely longitudinally striate throughout, with delicate punctures in interstices; T6 punctate rugulose dorsally, densely longitudinally striate laterodorsally, with scattered small punctures in interstices; length of T3 $0.93-1.03 \times$ length of T6; T5 longer than wide or slightly wider than long; S2-S4 sparsely and longitudinally striate, with delicate punctures in interstices; S5-S6 densely longitudinally striate, with delicate punctures in interstices; prominent longitudinal median carina present on S2-S5.

Male. Differing from female as follows: body length 4.08-6.26 mm $(\mathrm{n}=20)$; A1 brown, remainder of antenna dark brown; T4-T6 densely longitudinally striate throughout, with numerous delicate punctures in interstices; T7 punctate rugulose throughout; T6 slightly longer than wide; length of T6 1.42-1.84x length of T7; T7 subtriangular, apex pointed (Plate 24B, C); length of T7 1.43-1.87x length of S7; S7 longitudinally punctate rugulose; prominent longitudinal median carina present on S2-S6. 


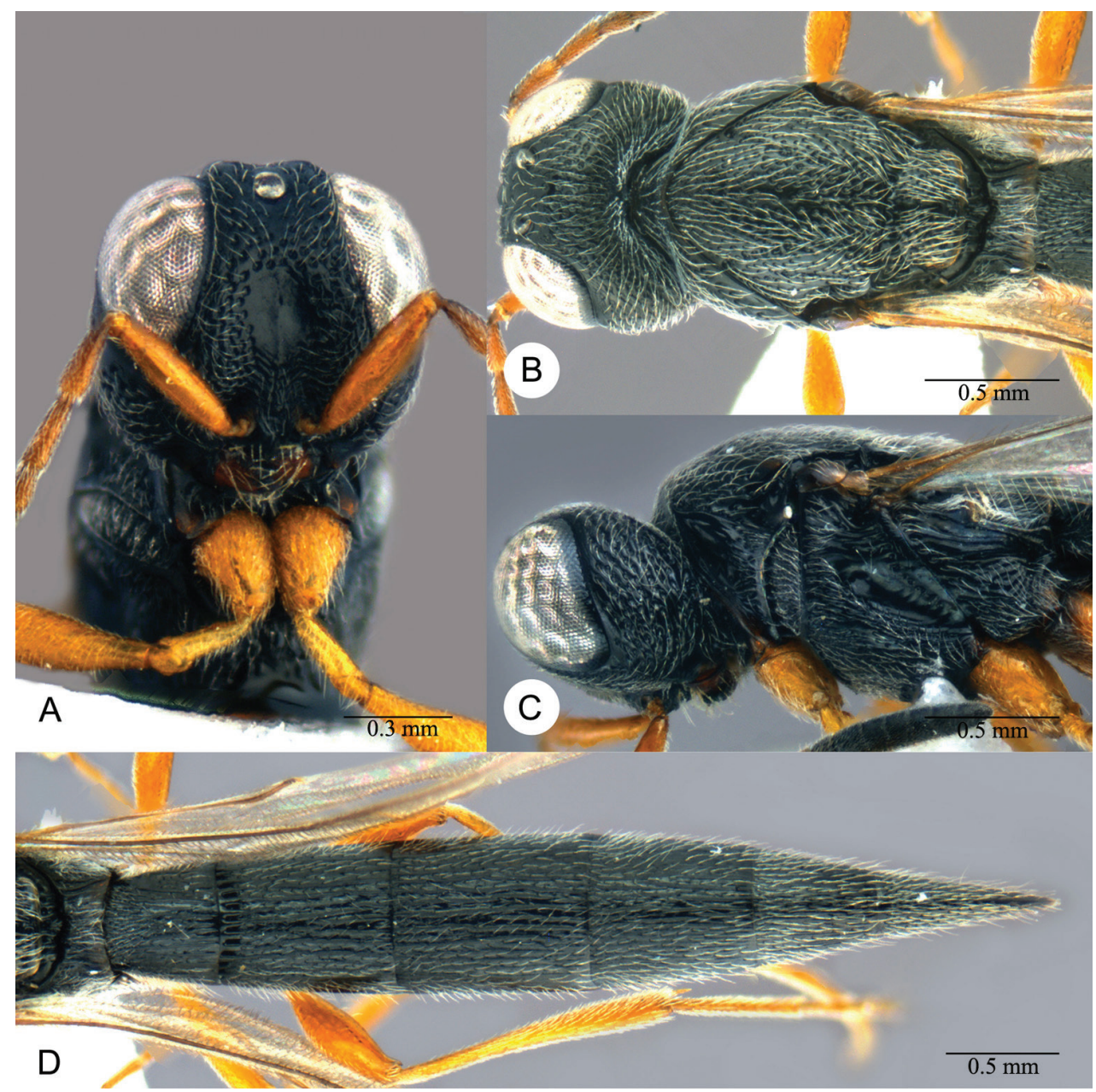

Plate 23. Macroteleia emarginata Dodd, female from Guangdong, Mt. Nankun. A Head, anterior view B Head and mesosoma, dorsal view C Head and mesosoma, lateral view D Head, anterior view E Metasoma, dorsal view.

Distribution. China (Fujian, Hunan, Guangdong, Hainan, Guizhou, Yunnan); Malaysia. Link to distribution map [http://hol.osu.edu/map-large.html?id=4802].

Material Examined. Other material. CHINA: 1 , Zhejiang, Mt. Fengyang, $27^{\circ} 56^{\prime} \mathrm{N}, 119^{\circ} 12^{\prime} \mathrm{E}, 18$. VIII.2003, Wuqing Fang, SCAU 000324 (SCAU); 1 O , Fujian, Huangzhulin Nature Reserve, $26^{\circ} 12^{\prime} \mathrm{N}, 118^{\circ} 51^{\prime} \mathrm{E}, 5 . V I I .2005$, Jingxian Liu, SCAU 000325 (SCAU); $1 \overbrace{}^{\lambda}$, Hunan, Nanyue, Mt. Heng, $27^{\circ} 15^{\prime} \mathrm{N}, 112^{\circ} 43^{\prime} \mathrm{E}$, 4.IX.1980, Xinwang Tong, SCAU 000326 (SCAU); 1 q, Hunan, Nanyue, Mt. Heng, $27^{\circ} 15^{\prime} \mathrm{N}, 112^{\circ} 43^{\prime} \mathrm{E}$, 5.IX.1980, Xinwang Tong, SCAU 000327 (SCAU); 2 $q+14 \delta^{\top}$, Hunan, Mangshan Nature Reserve $24^{\circ} 56^{\prime} \mathrm{N}, 112^{\circ} 69^{\prime} \mathrm{E}$, 13.VIII.2010, sweeping, Huayan Chen, SCAU 000328-000343 (SCAU); 1 + + 1 §, Guangdong, 


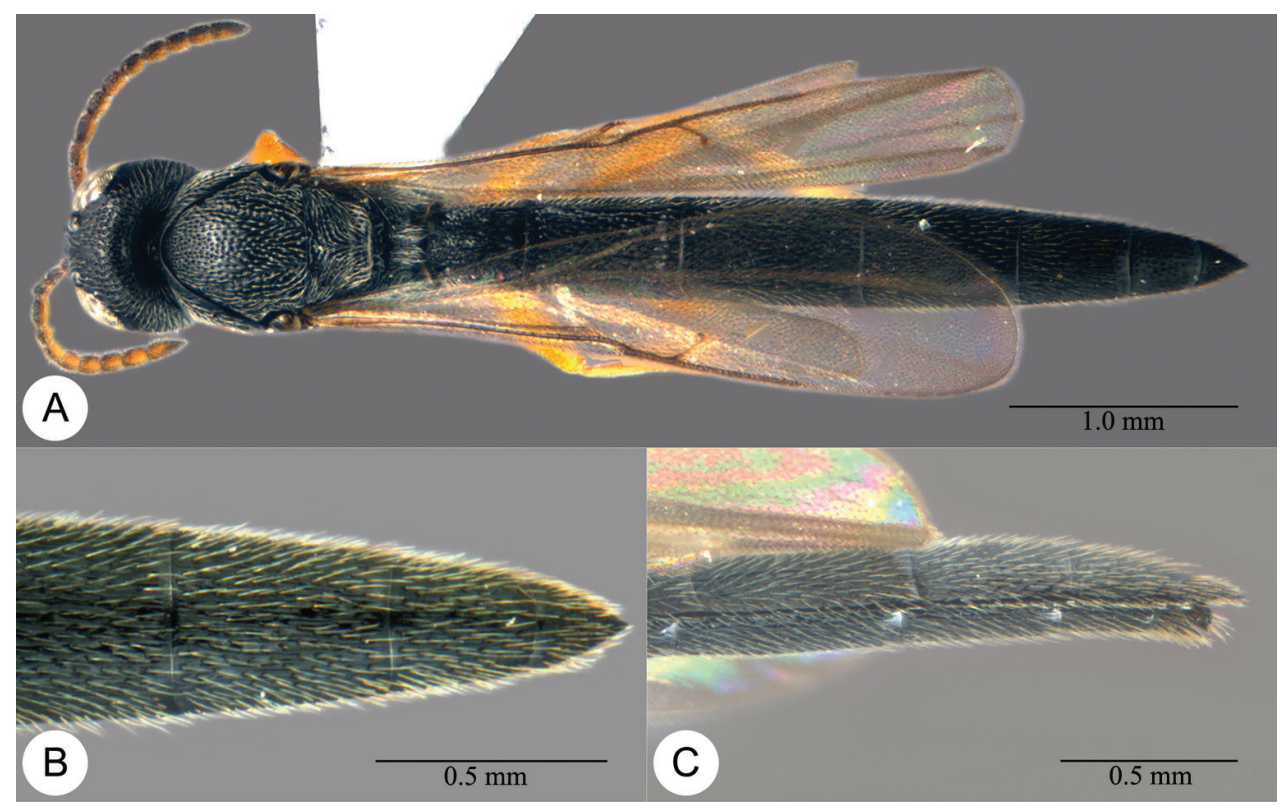

Plate 24. Macroteleia emarginata Dodd, male from Hunan, Mangshan Nature Reserve. A Dorsal habitus B Apex of metasoma, dorsal view C Apex of metasoma, lateral view.

Chebaling National Nature Reserve, $24^{\circ} 43^{\prime} \mathrm{N}, 14^{\circ} 14^{\prime} \mathrm{E}$, 23-28.VII.2008, Zaifu Xu, SCAU 000344, 000345 (SCAU); 1 क, Guangdong, Nanling National Nature Reserve, $24^{\circ} 54^{\prime} \mathrm{N}, 113^{\circ} 00^{\prime} \mathrm{E}$, 5.VI.2010, Huayan Chen, SCAU 000346 (SCAU); 1 \%, Guangdong, Nanling National Nature Reserve, $24^{\circ} 54^{\prime} \mathrm{N}, 113^{\circ} 00^{\prime} \mathrm{E}, 9-18 . V I I .2004$, Juanjuan Ma, SCAU 000347 (SCAU); 1 + , Guangdong, Mt. Nankun, 2337.941'N, $113^{\circ} 50.182^{\prime} \mathrm{E}, 12 . \mathrm{V} .2004$, Zaifu Xu, SCAU 000348 (SCAU); 1 ㅇ, Guangdong, Mt. Nankun, $23^{\circ} 37.941^{\prime} \mathrm{N}, 113^{\circ} 50.182^{\prime} \mathrm{E}, 2-3 . V I I .2005$, Zaifu Xu, SCAU 000349 (SCAU); 3 क, Guangdong, Mt. Nankun, $23^{\circ} 37.941$ 'N, $113^{\circ} 50.182^{\prime} \mathrm{E}, 15 . V I .2007$, Zaifu Xu, SCAU 000350-000352 (SCAU); 1 q, Guangdong, Mt. Nankun, $23^{\circ} 37.941^{\prime} \mathrm{N}, 113^{\circ} 50.182^{\prime} \mathrm{E}, 6 . \mathrm{V} .2010$, Jingxian Liu, SCAU 000353 (SCAU); 1 우, Guangdong, Mt. Nankun, $23^{\circ} 37.941$ 'N, $113^{\circ} 50.182^{\prime} E, 23 . V .2010$, Zaifu Xu, SCAU

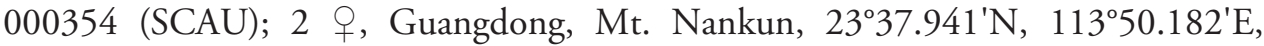
540m, 4.VI.2011, yellow pan trap, Huayan Chen, SCAU 000355, 000356 (SCAU);

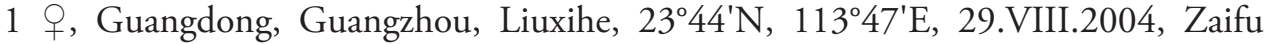
Xu, SCAU 000357 (SCAU); 1 ồ, Hainan, Mt. Diaoluo, 18³9'N, 10953'E, 1-2. VI.2007, Jingxian Liu, SCAU 000358 (SCAU); 2 , , Guizhou, Guiyang Forest Park, $26^{\circ} 33^{\prime} \mathrm{N}, 106^{\circ} 44^{\prime} \mathrm{E}, 23 . \mathrm{IX} .2007$, Cuihong Xie, SCAU 000359, 000360 (SCAU);

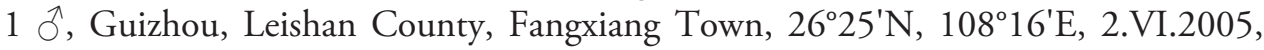
Jingxian Liu, SCAU 000361 (SCAU); 1 क, Guizhou, Leigongshan National Nature Reserve, Xiaodanjiang, 255' $\mathrm{N}, 108^{\circ} 24^{\prime} \mathrm{E}, 150 \mathrm{~m}$, 4.VI.2005, Jingxian Liu, SCAU 000362 (SCAU); 1 Oे, Guizhou, Leigongshan National Nature Reserve, Xiaodanjiang, $25^{\circ} 53^{\prime} \mathrm{N}, 108^{\circ} 24^{\prime} \mathrm{E}, 150 \mathrm{M}, 4 . \mathrm{VI} .2005$, Hongying Zhang, SCAU 000363 (SCAU); 


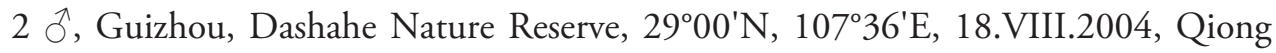
Wu, SCAU 000364, 000365 (SCAU); 1 ô, Yunnan, Nabanhe Basin National Nature Reserve, $22^{\circ} 15.474^{\prime} \mathrm{N}, 100^{\circ} 36.322^{\prime} \mathrm{E}, 892 \mathrm{M}, 19-23$.VII.2011, yellow pan trap, Zaifu $\mathrm{Xu}$, SCAU 000366 (SCAU).

Comments. We did not examine the holotype of this species. The identification is based upon the careful description provided by Alan Dodd in the original publication.

\section{Macroteleia flava sp. $\mathbf{n}$.}

urn:Isid:zoobank.org:act:8594A627-E6B3-4740-908D-676EFF342C08

http://species-id.net/wiki/Macroteleia_flava

Plates 25-26

Description. Female. Body length 4.35-5.76 mm ( $\mathrm{n}=5)$.

Color. Head and mesosoma orange yellow; base of T1 and T6 variably dark brown to black, otherwise orange yellow; mandible dark brown; palpi yellow; legs yellow throughout; A1-A6 brown, A7-A12 black; fore wing hyaline.

Head. Transverse in dorsal view, $1.40-1.44 \times$ as wide as long, slightly wider than mesosoma; OOL short, 0.14-0.31× minimum ocellar width; POL 1.20-1.31× LOL; occipital carina weakly continuous or interrupted medially; central keel absent (Plate 25C); medial frons punctate rugulose ventrally, irregularly smooth dorsally; ventrolateral frons punctate rugose; frons below median ocellus densely punctate; vertex densely punctate, with punctures in part contiguous; gena punctate rugose; length of A3 equal to length of A2.

Mesosoma. Cervical pronotal area densely punctate; dorsal pronotal area punctate rugose; lateral pronotal area smooth anteriorly, punctate rugulose posteriorly; netrion punctate rugulose; notaulus narrow, but distinctly foveolate; middle lobe of mesoscutum densely punctate, becoming denser anteriorly and at posterior end; lateral lobes of mesoscutum densely punctate throughout; mesoscutellum densely punctate throughout; metascutellum distinctly transverse (Plate $25 \mathrm{D}$ ), posterior margin slightly pointed medially, longitudinally carinate; propodeum continuous medially (Plate 25D), not divided into two separated lobes, posterior margin narrowly notched medially, each side with several irregular longitudinal carinae medially, otherwise punctate rugulose, covered by dense, recumbent, white setae; upper mesepisternum with a row of fine longitudinal carinae below subalar pit; lower mesepisternum punctate rugulose; mesopleural depression smooth (Plate 25E); metapleuron longitudinally striate throughout.

Legs. Slender; hind femur weakly swollen, $3.60-4.39 \times$ as long as its maximum width; hind tibia without spines over outer surface; hind basitarsus $11.66-12.83 \times$ as long as its maximum width.

Wings. Apex of fore wing extending from as far as posterior third to posterior margin of T4; R 1.76-2.25× as long as r-rs, R1 1.39-2.00× length of R.

Metasoma. Posterior margin of transverse sulcus on T2 strongly convex (Plate 25F); sublateral tergal carinae developed on T1-T3; T1 densely longitudinally striate 


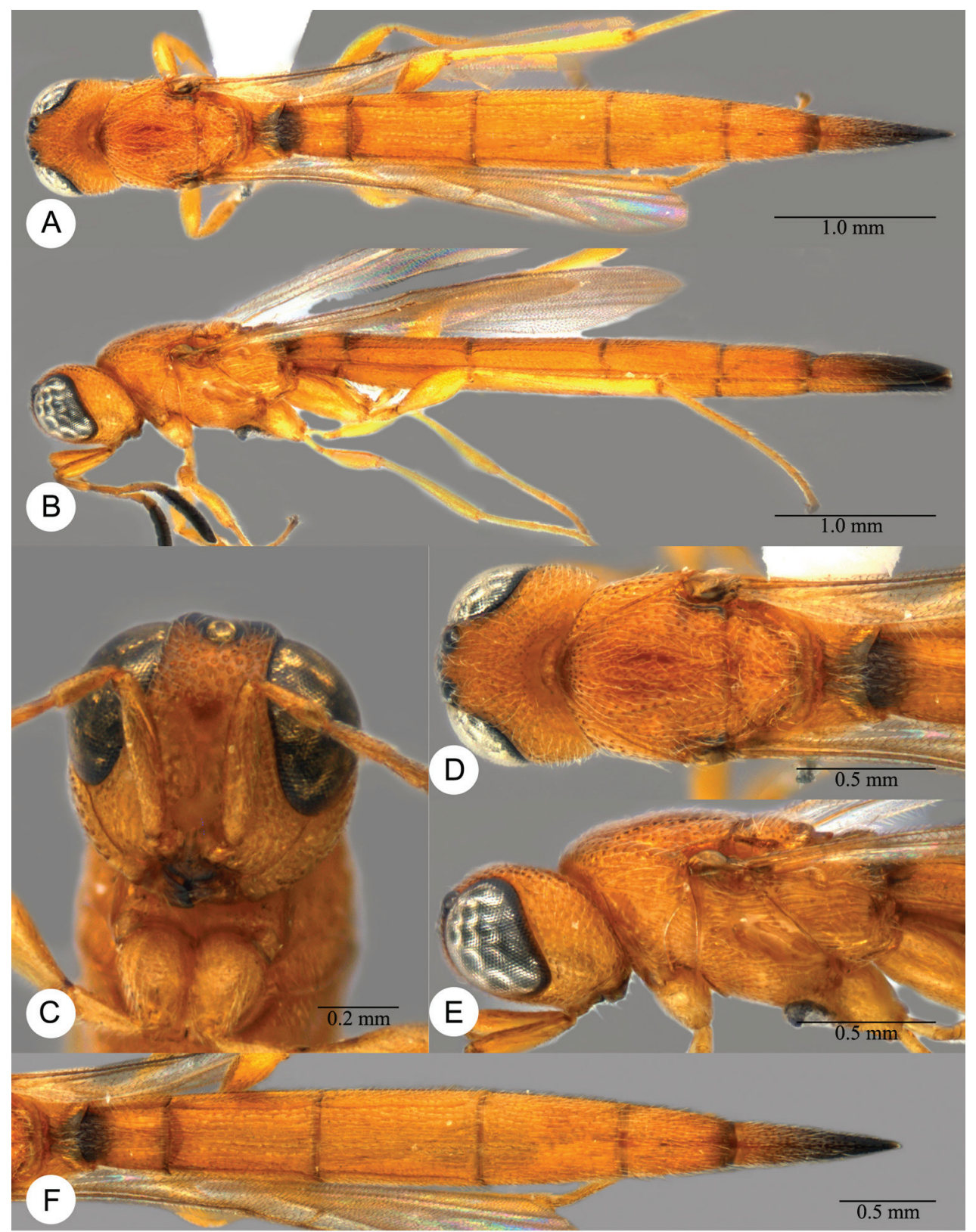

Plate 25. Macroteleia flava sp. n., holotype, female. A Dorsal habitus B Lateral habitus. C Head, anterior view D Head and mesosoma, dorsal view E Head and mesosoma, lateral view F Metasoma, dorsal view.

medially, with scattered punctures in interstices anteriorly, rugulose laterally; T2-T3 densely longitudinally striate medially, with delicate punctures in interstices, punctate rugulose laterally; T4-T5 densely finely longitudinally striate throughout, with delicate punctures in interstices; T6 finely punctate dorsally, densely longitudinally striate 


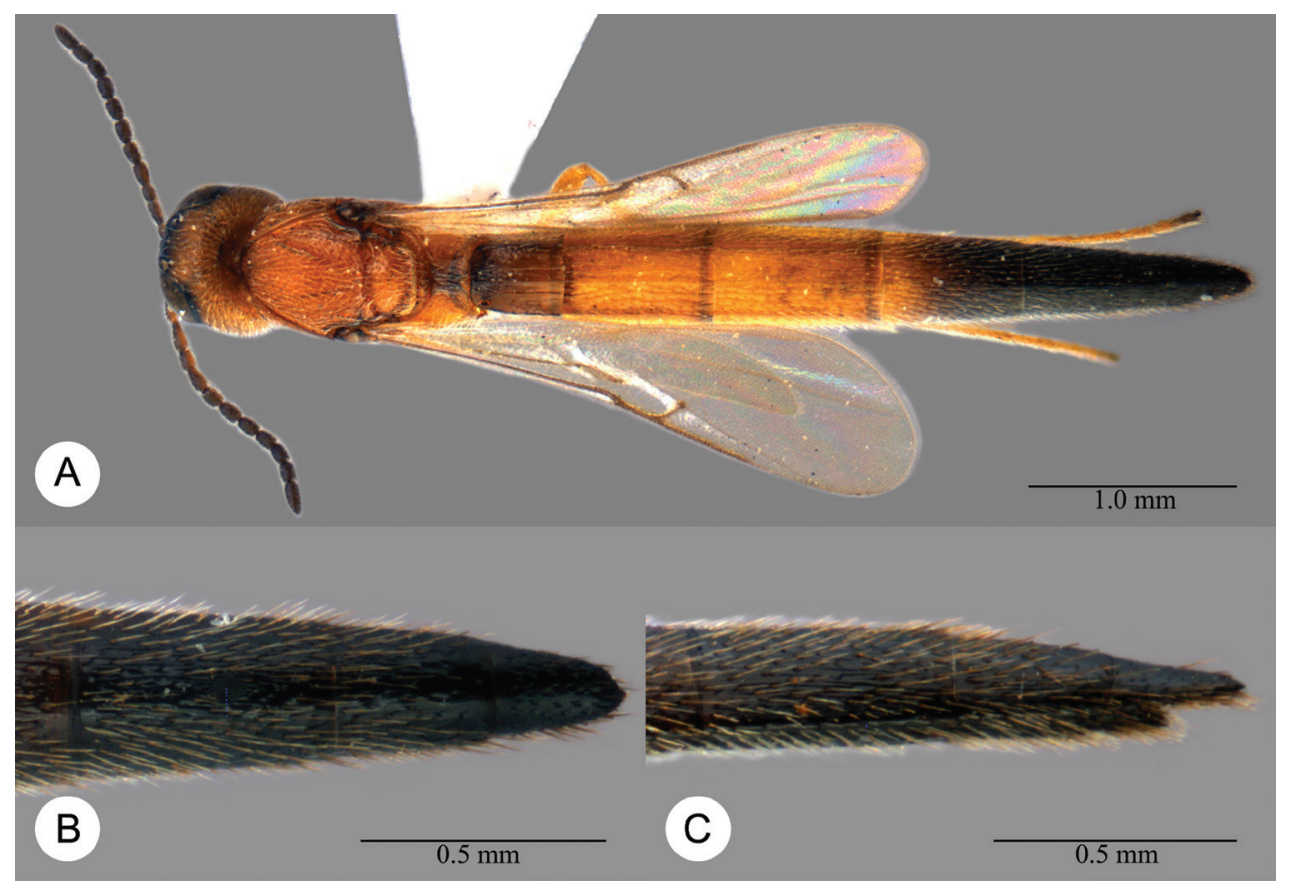

Plate 26. Macroteleia flava sp. n., paratype, male. A Dorsal habitus B Apex of metasoma, dorsal view C Apex of metasoma, lateral view.

laterally, with scattered small punctures in interstices; length of T3 $0.83-0.99 \times$ length of T6; T5 distinctly longer than wide; S2-S4 longitudinally striate, with finely punctate rugulose interstices; $S 5-S 6$ longitudinally striate, with finely punctate interstices; prominent longitudinal median carinae present on S2-S5.

Male. Differing from female as follows: body length 4.55-5.26 mm ( $\mathrm{n}=6)$; head brown; mesosoma darker than female; metasoma variably brown to black; A1 yellow, remainder of antenna brown to dark brown; T1-T3 densely longitudinally striate medially, with delicate punctures in interstices, rugulose laterally; T4-T7 longitudinally punctate rugulose; T6 slightly longer than wide; length of T6 1.09-1.27× length of T7; T7 subtriangular, apex pointed (Plate 26B, 26C); length of T7 1.75-2.29× length of S7; S2-S5 longitudinally striate, with delicate punctures in interstices; S6-S7 longitudinally punctate rugulose.

Diagnosis. The body shape, color and size of $M$. flava is similar to $M$. rufa and $M$. chandelii. It differs from them in that metascutellum is distinctly transverse (triangular in $M$. rufa, tongue-like in M. chandelii), and the propodeum is continuous medially, not divided into two separated lobes (divided into two subtriangular lobes in the latter two species).

Etymology. The name flava refers to orange yellow body color of this species and is used as an adjective.

Distribution. China (Hebei, Hunan, Guangdong); Thailand. Link to distribution map [http://hol.osu.edu/map-large.html?id=320504]. 
Material examined. Holotype, + : CHINA: Hebei, Mt. Dongling, $40^{\circ} 02^{\prime} \mathrm{N}$, $115^{\circ} 27^{\prime}$ E, 11.VIII.2005, Ming Shi, SCAU 000078 (deposited in SCAU). Paratypes: 1 Oे, Hunan, Nanyue, Mt. Heng, $27^{\circ} 15^{\prime} \mathrm{N}, 112^{\circ} 43^{\prime} \mathrm{E}, 20 . \mathrm{VI} .1980$, Xinwang Tong, SCAU 000079 (SCAU); 1 ', Hunan, Nanyue, Mt. Heng, $27^{\circ} 15^{\prime} \mathrm{N}, 112^{\circ} 43^{\prime} \mathrm{E}$, 17.VIII.1980, Xinwang Tong, SCAU 000080 (SCAU); 1 , Hunan, Nanyue, Mt. Heng, $27^{\circ} 15^{\prime} \mathrm{N}, 112^{\circ} 43^{\prime} \mathrm{E}$, 4.IX.1980, Xinwang Tong, SCAU 000081 (SCAU); 1 ${ }^{\top}$, Hunan, Nanyue Town, $27^{\circ} 14^{\prime} \mathrm{N}, 112^{\circ} 44^{\prime} \mathrm{E}$, 8.IX.1980, Xinwang Tong, SCAU

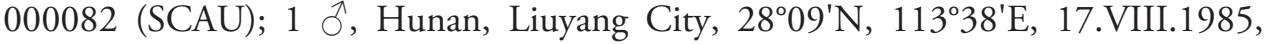
Xinwang Tong, SCAU 000083 (SCAU); 1 + , Guangdong, Meizhou, Fenxi Forestry Farm, $24^{\circ} 38^{\prime} \mathrm{N}, 116^{\circ} 47^{\prime} \mathrm{E}, 28 . V I I .2003$, Jianuan Zhou, SCAU 000084 (SCAU); 1 ${ }^{\top}$, Guangdong, Xinfeng, Mt. Yunji, $24^{\circ} 04^{\prime} \mathrm{N}, 114^{\circ} 10^{\prime} \mathrm{E}, 19 . \mathrm{VII} .2003$, Yanxia Song, SCAU 000496 (SCAU); 1 ð, Guangdong, Mt. Nankun, $23^{\circ} 37.941^{\prime} \mathrm{N}, 113^{\circ} 50.182^{\prime} \mathrm{E}$, 2.VII.2005, Zaifu Xu, SCAU 000085 (SCAU). THAILAND: 1 + , Chiang Mai: Maerim, 11.XI.2002, MT, R. A. Beaver, No. 25682 (RABC); 1 + , Chiang Mai: Maerim, 5-8.IV.2003, MT, R. A. Beaver, No. 27038 (RABC).

\section{Macroteleia gracilis sp. $\mathbf{n}$.}

urn:Isid:zoobank.org:act:FC1AC5B9-9F13-4AC7-9057-7DD106F227AB

http://species-id.net/wiki/Macroteleia_gracilis

Plates 27-28

Description. Male. Body length 4.76-6.05 mm ( $\mathrm{n}=4)$.

Color. Body black; mandible reddish brown; palpi yellow; hind coxa blackish, tarsi yellow, remainder of legs pale brown; A1 brown, remainder of antenna dark brown; fore wing hyaline.

Head. Transverse in dorsal view, $1.44-1.48 \times$ as wide as long, slightly wider than mesosoma; OOL short, $0.17-0.29 \times$ minimum ocellar width; POL 1.54-1.57× LOL; occipital carina weakly continuous medially, irregular punctate; central keel weakly developed, extending onto interantennal process (Plate 28A); medial frons punctate rugulose ventrally, with irregularly shaped smooth area dorsally; frons below median ocellus sparsely punctate medially, densely punctate laterally; vertex punctate rugulose; gena punctate rugose; length of A3 1.25-1.31× length of A2.

Mesosoma. Cervical pronotal area densely punctate; dorsal pronotal area punctate rugulose; lateral pronotal area smooth dorsally, punctate rugulose ventrally; netrion finely punctate rugulose; notaulus shallow, foveolate; middle lobe of mesoscutum densely punctate, sculpture becoming denser anteriorly; lateral lobes of mesoscutum densely finely punctate throughout; mesoscutellum densely finely punctate throughout; metascutellum transverse (Plate 28B), posterior margin slightly pointed medially, longitudinally carinate; propodeum continuous medially (Plate 28B), not divided into two separated lobes, posterior margin narrowly notched medially, each side with several irregular longitudinal carinae medially, otherwise punctate rugulose, covered by dense, recumbent, white setae; upper mesepisternum with a row of somewhat robust longitudinal carinae below subalar 


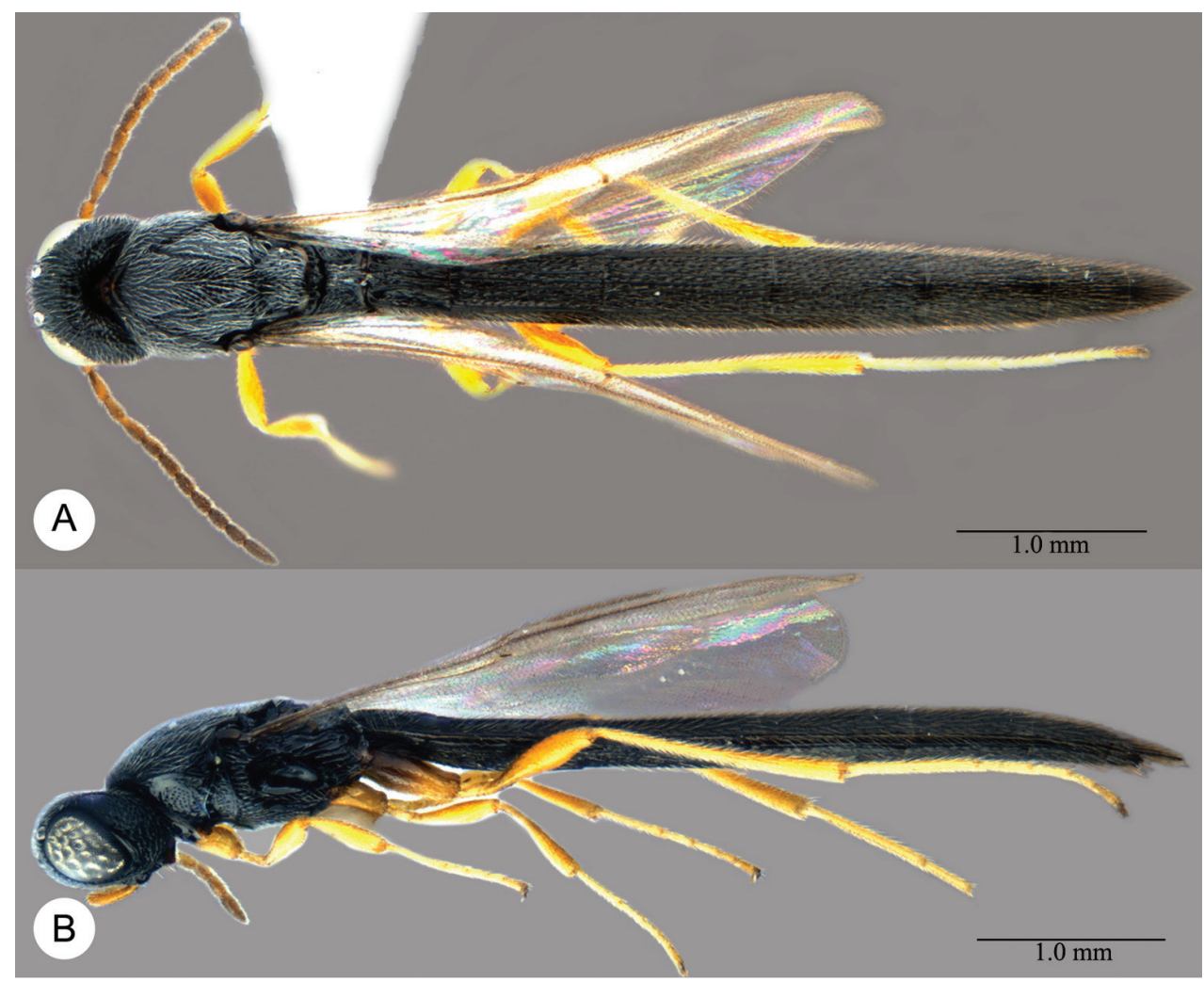

Plate 27. Macroteleia gracilis sp. n., holotype, male. A Dorsal habitus B Lateral habitus.

pit; lower mesepisternum variably smooth to punctate rugulose; mesopleural depression smooth (Plate 28C); metapleuron longitudinally striate throughout.

Legs. Slender; hind femur weakly swollen, $4.23-4.80 \times$ as long as its maximum width; hind tibia without spines over outer surface; hind basitarsus $12.60-14.00 \times$ as long as its maximum width.

Wings. Apex of fore wing extending from as far as posterior fourth of $\mathrm{T} 3$ to base of T4; R 2.06-2.46× as long as r-rs, R1 1.63-1.90× length of R.

Metasoma. Posterior margin of transverse sulcus on T2 slightly convex (Plate 28D); sublateral tergal carinae developed on T1-T3; T1-T2 sparsely and longitudinally striate medially, with delicate punctures in interstices, punctate rugulose laterally; T3-T6 densely longitudinally striate, with numerous delicate punctures in interstices; T7 punctate rugulose throughout, with longitudinal tendency; T6 distinctly longer than wide; length of T6 $0.93-1.18 \times$ length of T7; T7 subtriangular, apex pointed (Plate 28E, 28F); length of T7 2.50-3.22× length of S7; S2-S3 sparsely longitudinally striate, with numerous delicate punctures and irregular microsculptures in interstices; S4-S7 densely longitudinally striate, with numerous delicate punctures in interstices; prominent longitudinal median carina present on S2-S5. 


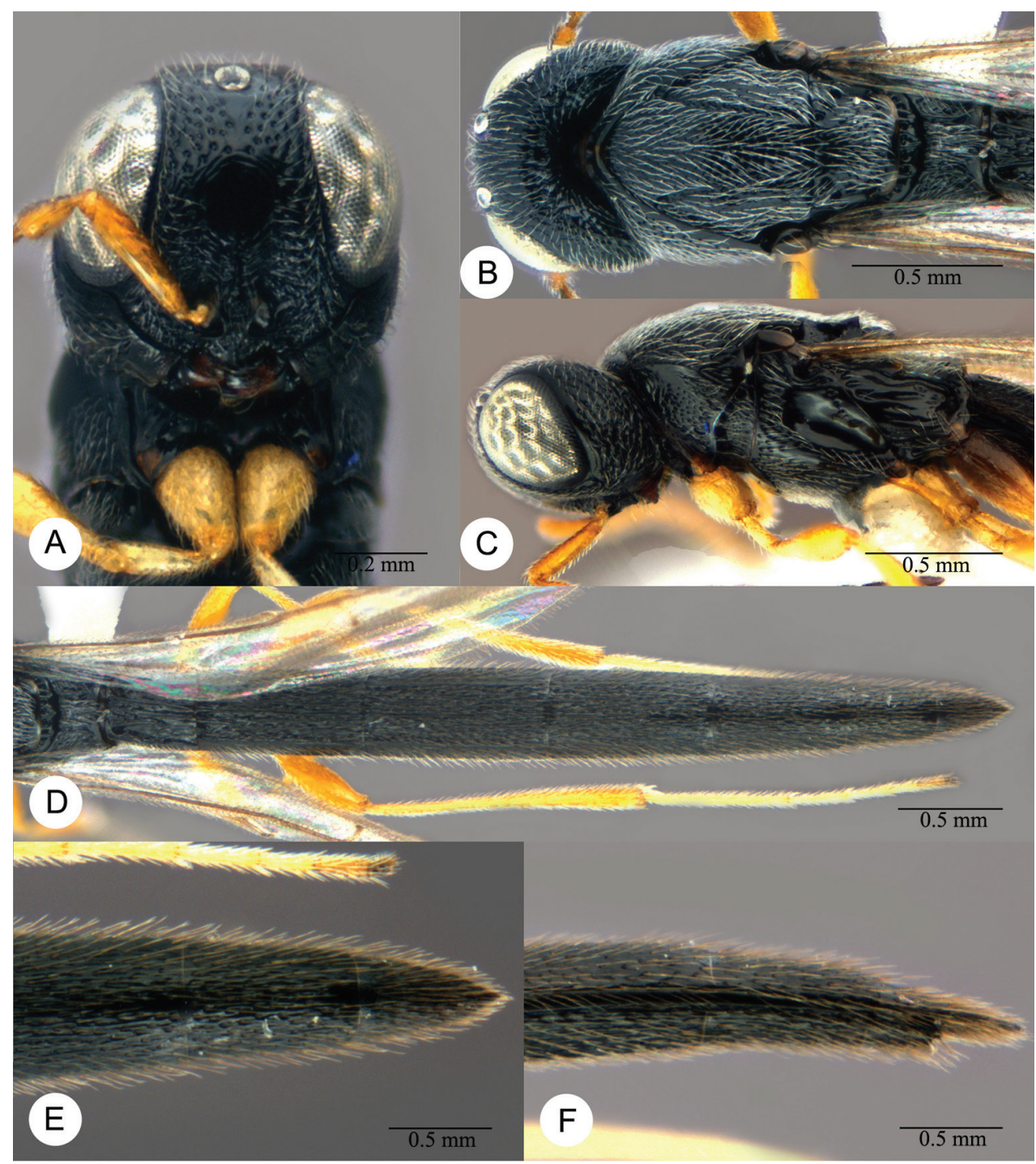

Plate 28. Macroteleia gracilis sp. n., holotype, male. A Head, anterior view B Head and mesosoma, dorsal view C Head and mesosoma, lateral view D Metasoma, dorsal view E Apex of metasoma, dorsal view F Apex of metasoma, lateral view.

Female. Unknown.

Diagnosis. The male of this species is similar to that of M. emarginata in size and color, but can be distinguished in having the metapleuron longitudinally striate throughout ( $M$. emarginata longitudinally striate dorsally, punctate rugulose ventrally); and the length of T7 2.50-3.22× length of S7 (distinctly shorter, 1.42-1.87× in M. emarginata).

Etymology. The name gracilis refers to the slender body of this species and is used as an adjective. 
Distribution. China (Guangdong). Link to distribution map [http://hol.osu.edu/ map-large.html?id=320505].

Material examined. Holotype, ô: CHINA: Guangdong, Mt. Nankun, $23^{\circ} 37.941$ 'N, $113^{\circ} 50.182^{\prime} \mathrm{E}, 1 . \mathrm{VI} .2009$, Huayan Chen, SCAU 000053 (deposited in

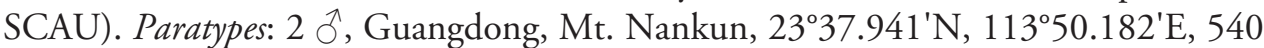
m, 4.VI.2011, yellow pan trap, Huayan Chen, SCAU 000054, 000055 (SCAU); 1 ○, Guangdong, Shimen National Forest Park, 2337.287'N, 11351.267'E, 4.VI.2009, Huayan Chen, SCAU 000056 (SCAU).

\section{Macroteleia indica Saraswat \& Sharma}

http://species-id.net/wiki/Macroteleia_indica

Plates 29-35

Macroteleia indica Saraswat and Sharma 1978: 11 (original description); Mani and Sharma 1982: 171 (description); Saraswat 1982: 346 (description of male); Mukerjee 1994: 4 (color variation).

Macroteleia cebes Lê 2000: 52, 54, 331 (original description, keyed), syn. n. Macroteleia dones Lê 2000: 52, 53, 57, 333 (original description, keyed), syn. n. Macroteleia domes Lê 2000: 52 (keyed, misspelling).

Description. Female. Body length 3.13-4.76 mm (n=20).

Color. Head yellow or orange throughout, or dark orange, becoming darker dorsally; mesosoma yellow or orange, becoming darker dorsally; base of T1, T5 and T6 brown to black, remainder of metasoma yellow or orange; mandible yellow with teeth black; palpi yellow; legs yellow throughout; A1 yellow, A2-A6 yellow or brown, remainder of antenna black; fore wing hyaline.

Head. Transverse in dorsal view, $1.26-1.40 \times$ as wide as long, slightly wider than mesosoma; OOL short, $0.10-0.40 \times$ times minimum diameter of lateral ocellus; POL 1.14-1.36x LOL; occipital carina interrupted medially; central keel absent (Plate $34 \mathrm{~A})$; medial frons obliquely strigose ventrally, irregularly smooth dorsally; ventrolateral frons punctate rugose; frons below median ocellus densely punctate, punctures in part contiguous; vertex densely punctate, interspaces in part with microsculpure; gena coarsely punctate rugose; length of A3 $0.80-0.94 \times$ length of A2.

Mesosoma. Cervical pronotal area densely punctate; dorsal pronotal area densely coarsely punctate; lateral pronotal area smooth on upper anterior angle, punctate rugose posteriorly; netrion punctate rugulose; notaulus shallow, irregularly foveolate; middle lobe of mesoscutum moderately punctate; lateral lobes of mesoscutum densely punctate; mesoscutellum densely punctate throughout; metascutellum transverse (Plates 31D, $32 \mathrm{C}, 34 \mathrm{~B})$, posterior margin slightly pointed medially, longitudinally carinate; propodeum continuous medially (Plates 31D, 32C, 34B), not divided into two separated lobes, posterior margin narrowly notched medially, each side with several irregular longitudinal carinae submedially, otherwise punctate rugulose, covered by dense, recumbent, white 


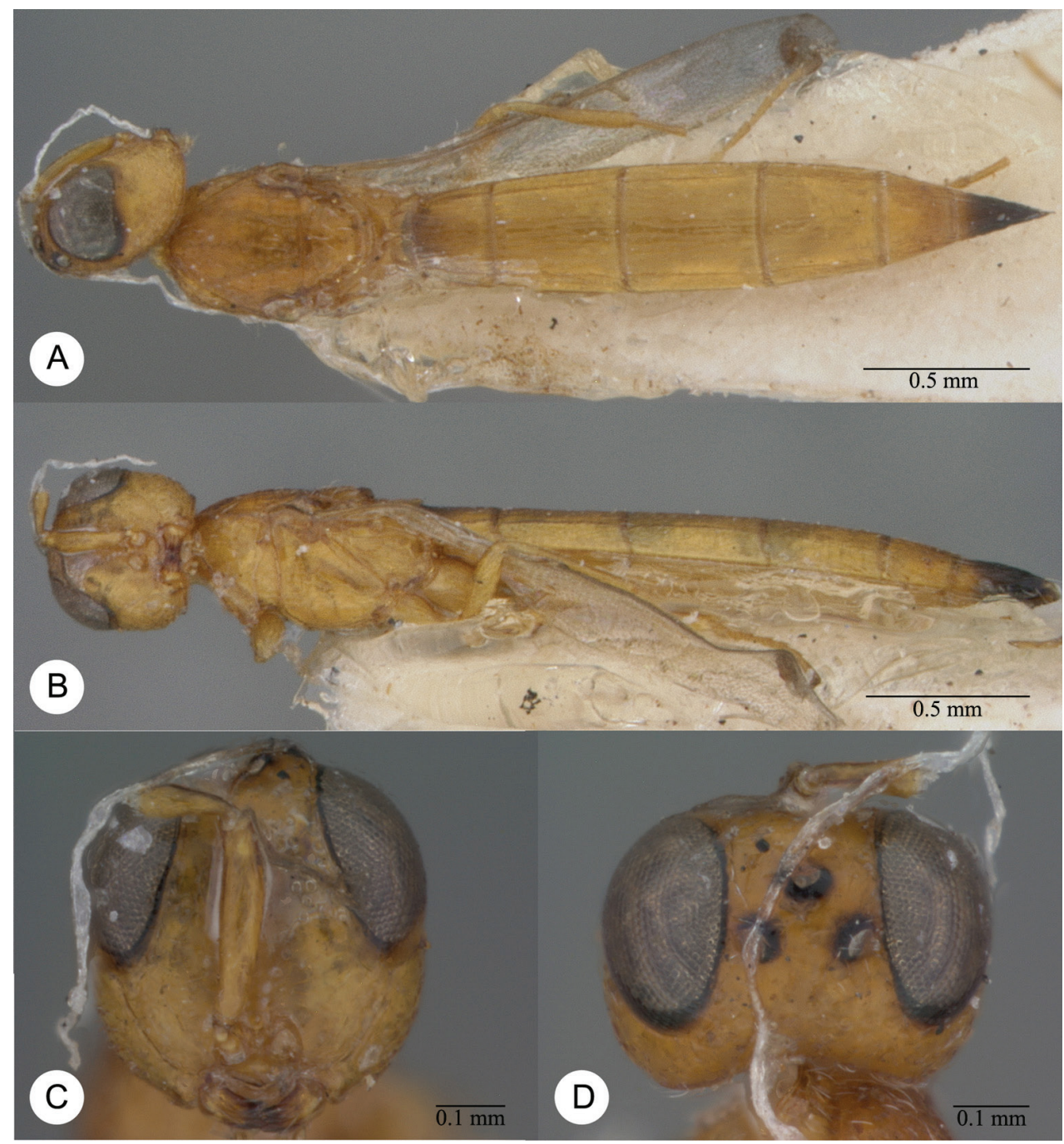

Plate 29. Macroteleia indica Saraswat \& Sharma, holotype, female. A Dorsal habitus B Lateral habitus C Head, anterior view D Head, dorsal view.

setae; upper mesepisternum with a row of weak longitudinal carinae or a ledge below subalar pit; lower mesepisternum punctate rugulose; mesopleural depression smooth (Plates 31E, 32D, 34C); metapleuron longitudinally striate throughout.

Legs. Slender; hind femur weakly swollen, $3.75-4.33 \times$ as long as its maximum width; hind tibia without spines over outer surface; hind basitarsus $10.00-13.43 \times$ as long as its maximum width.

Wings. Apex of fore wing extending as far as posterior margin of $\mathrm{T} 4$ to posterior margin of T5; R 1.70-2.25× as long as r-rs, R1 1.57-2.05× length of R.

Metasoma. Posterior margin of transverse sulcus on T2 slightly convex (Plates 31F, 34D); sublateral tergal carinae developed on T1-T4; T1-T4 densely longi- 


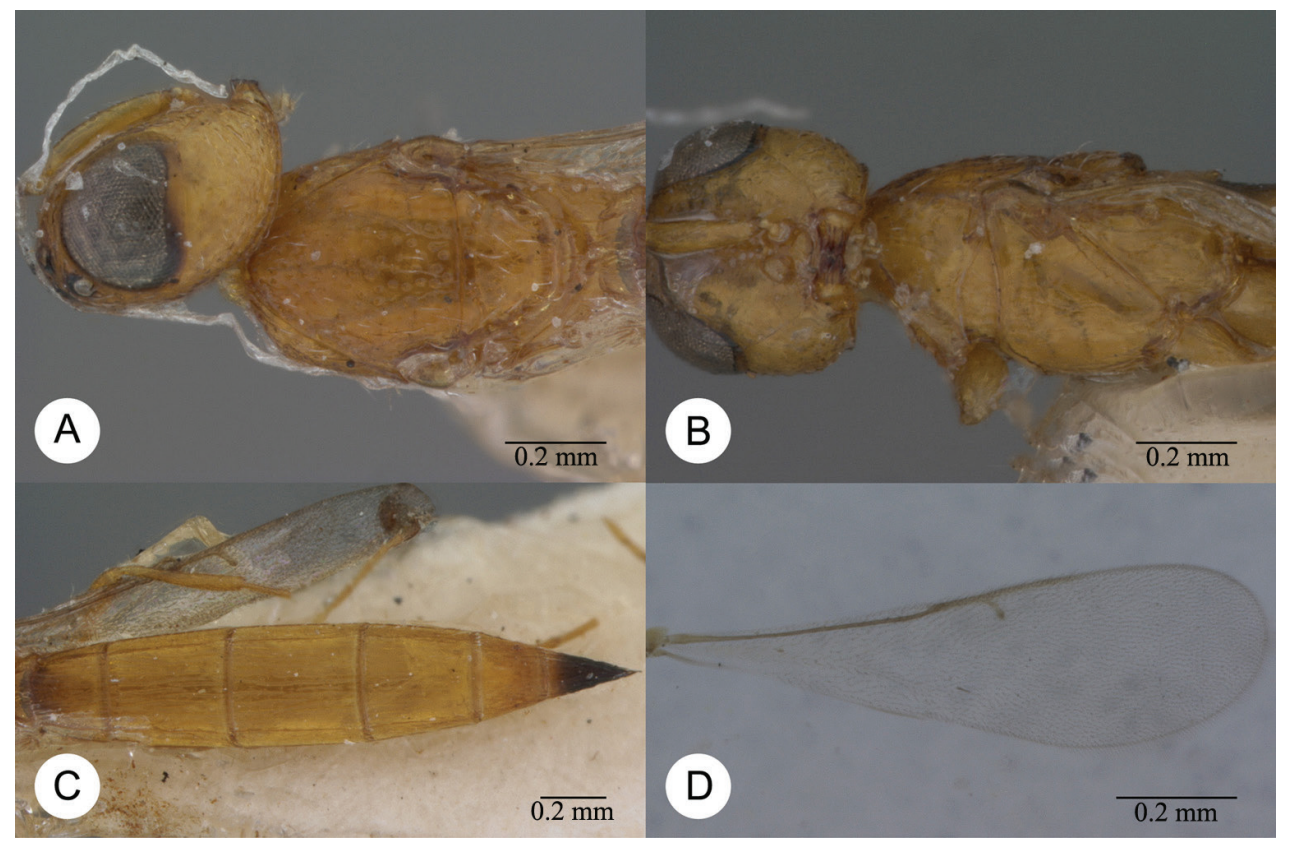

Plate 30. Macroteleia indica Saraswat \& Sharma, holotype, female. A Head and mesosoma, dorsal view B Head and mesosoma, lateral view C Metasoma, dorsal view D Fore wing.

tudinally striate medially, with scattered delicate punctures in interstices, punctate rugulose laterally; T5 densely longitudinally striate throughout, with delicate punctures in interstices; T6 finely punctate dorsally, densely longitudinally striate laterally, with scattered small punctures in interstices; length of T3 1.11-1.39x length of T6; T5 distinctly wider than long; S2-S3 moderately longitudinally striate, with punctate rugulose sculpture in interstices; S4-S6 densely longitudinally striate, with delicate punctures in interstices; prominent longitudinal median carina present on S2-S5.

Male. Differing from female as follows: body length 2.94-4.17 mm ( $\mathrm{n}=20)$; body darker than female; A1 pale brown, remainder of antenna brown to dark brown, becoming darker apically; T5-T6 densely longitudinally striate, with numerous delicate punctures in interstices; T7 punctate rugulose throughout; T6 distinctly wider than long; length of T6 1.82-2.57× length of T7; T7 transverse, apex truncate (Plate 35B); length of T7 $0.73-0.91 \times$ length of S7; S6-S7 longitudinally punctate rugulose; prominent longitudinal median carina present on S2-S6.

Diagnosis. This species is similar to $M$. flava in body shape and color, but can be distinguished by its smaller size and its T5 distinctly wider than long (T5 distinctly longer than wide in $M$. flava).

Distribution. China (Zhejiang, Taiwan, Fujian, Hunan, Guangdong, Hainan, Guangxi, Yunnan); India; Vietnam. Link to distribution map [http://hol.osu.edu/ map-large.html?id=4819]. 


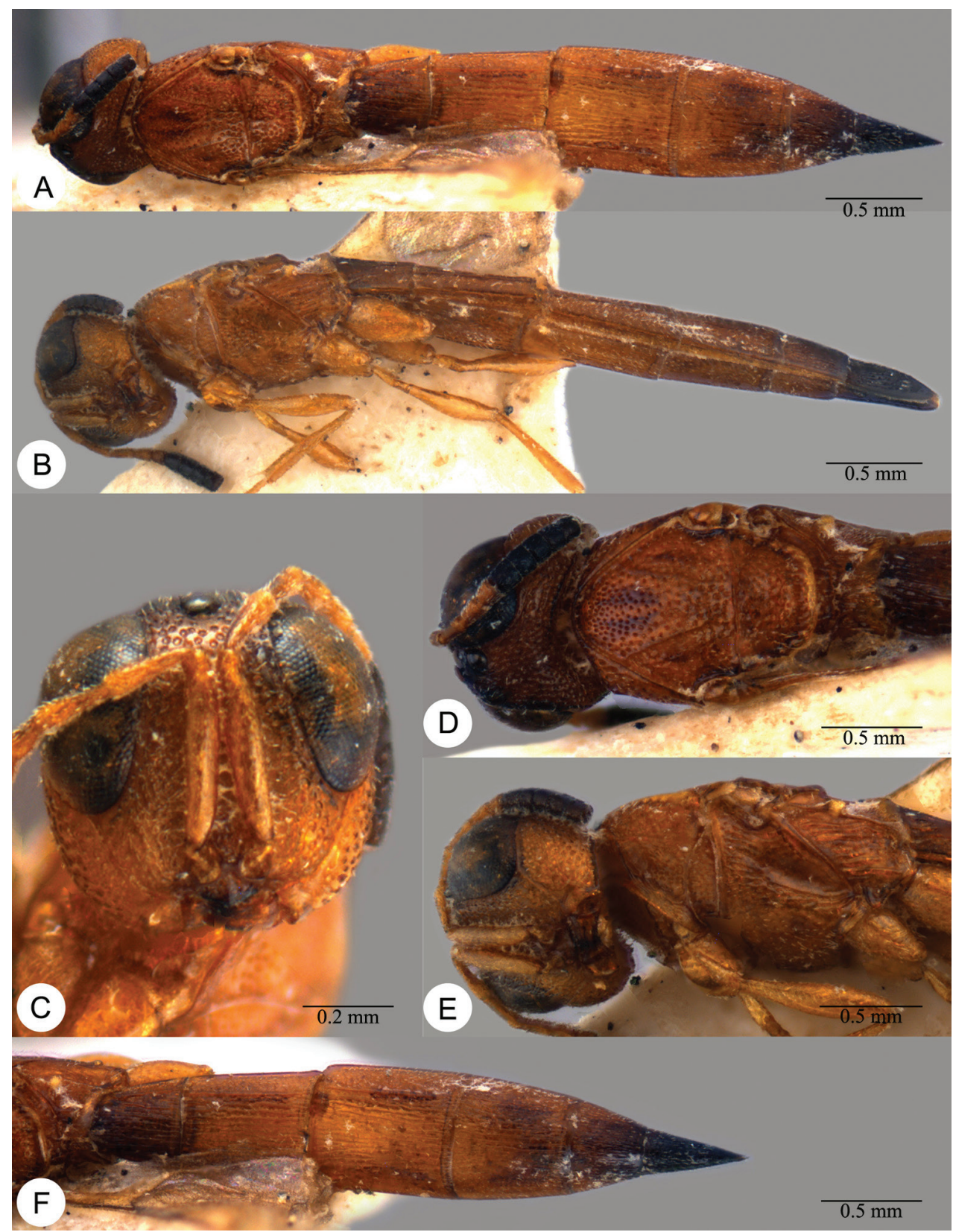

Plate 31. Macroteleia cebes Kozlov \& Lê, holotype, female. A Dorsal habitus B Lateral habitus C Head, anterior view $\mathbf{D}$ Head and mesosoma, dorsal view E Head and mesosoma, lateral view F Metasoma, dorsal view.

Material examined. Holotype of M. indica, $q$ : INDIA: "North-Bengal Survey, School of Entomology, St. John's College, Agra-282002, India, 16.1. Alipur Duar, M.S. Mani \& Party, 1-19.IV.1976", "Holotype", "Macroteleia indica Sharma, NO. SA+1, O” (deposited 


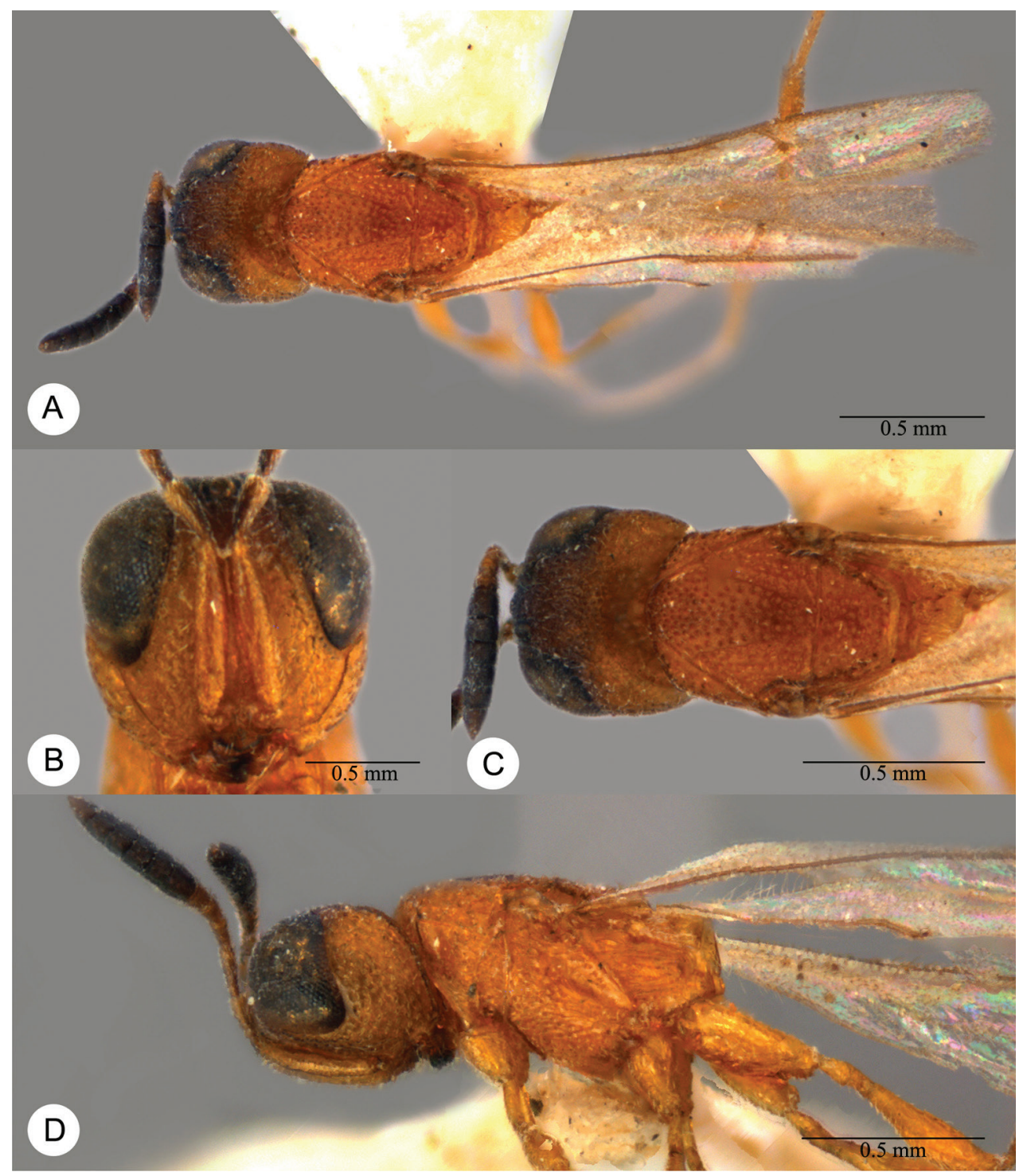

Plate 32. Macroteleia dones Kozlov \& Lê, holotype, female. A Dorsal habitus B Head, anterior view C Head and mesosoma, dorsal view D Head and mesosoma, lateral view.

in USNM). Holotype of M. cebes, + , VIETNAM: "Thuong Tien, HSB [=Hoa Binh], rice field, 8.XI.1978, Lê Xuan Hue", "Holotypus 9 Macroteleia cebes Kozlov et Lê 84” (deposited in IEBR). Holotype of $M$. dones,, , VIETNAM: "Suoi Trai, Tra Mi, QN-DN [=Quang Nam-Da Nang], on grass in forest, 17.IV.1983, LêXuan Hue", "Holotypus + Macroteleia dones Kozlov et Lê 84" (deposited in IEBR). Paratypes of M. dones, 1 q, VIETNAM: "next to An Khe, on grass in forest, 4.XII.1978, Lê Xuan Hue" (IEBR); 1 đ,, "Son La Province, on grass, 2.V.1986, V. Triapitzin", "Paratypus Macroteleia dones sp. n.” (IEBR). 


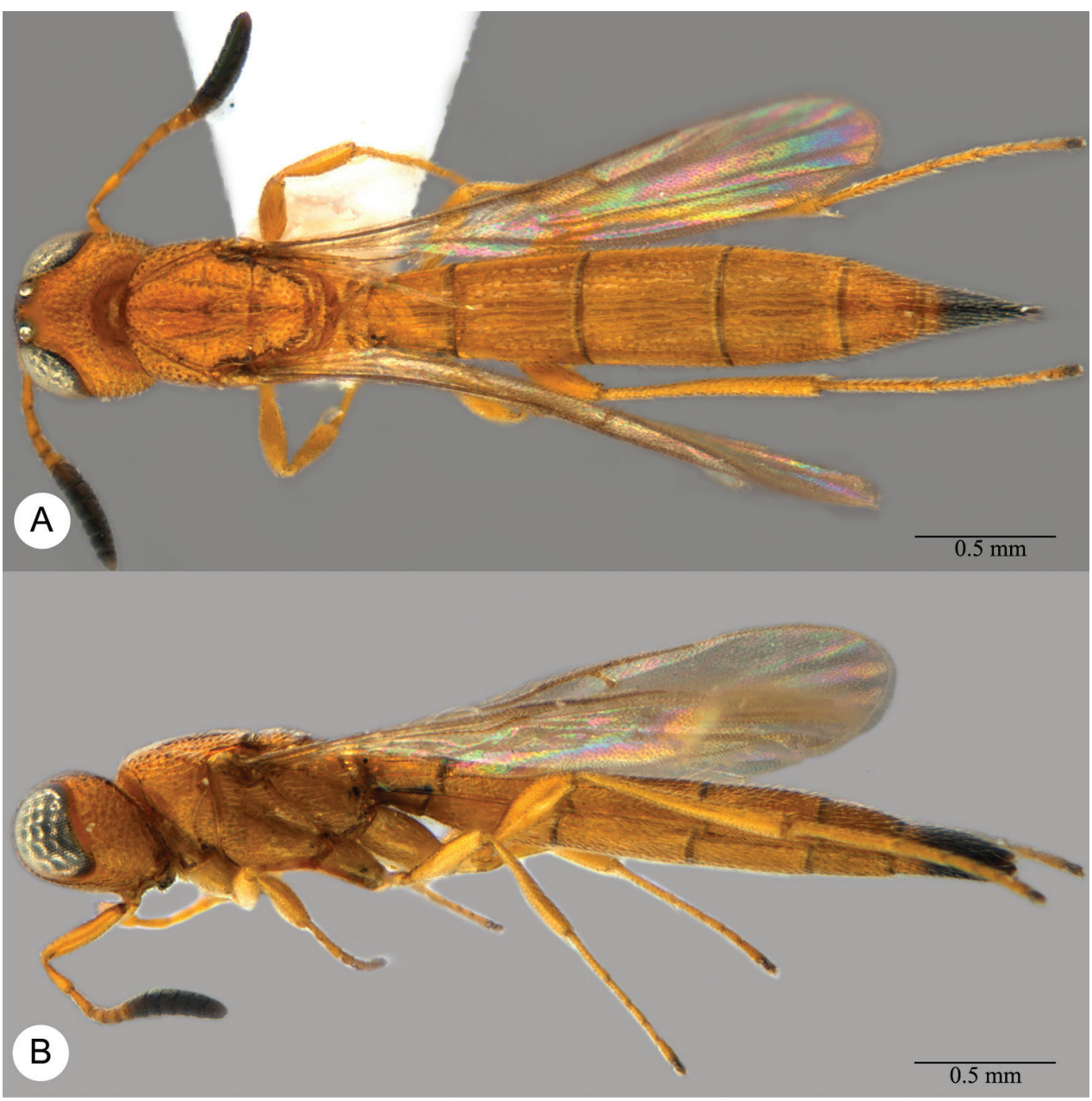

Plate 33. Macroteleia indica Saraswat \& Sharma, female from Hainan, Baisha, Mt. Jiujialing. A Dorsal habitus B Lateral habitus.

Other material. CHINA: $3+2{ }^{\lambda}$, Zhejiang, Hangzhou, West Lake, $30^{\circ} 15^{\prime} \mathrm{N}$, $120^{\circ} 07^{\prime} E$, 25.VIII.2003, Qiong Wu, SCAU 000367-000371 (SCAU); 1 क , Fujian, Mt. Wuyi, $27^{\circ} 43^{\prime} \mathrm{N}, 117^{\circ} 42^{\prime} \mathrm{E}, 22-25 . V I I I .2007$, Jie Zeng, SCAU 000372 (SCAU); 1 ${ }^{\top}$, Taiwan, Kenting National Park, $21^{\circ} 57^{\prime} \mathrm{N}, 120^{\circ} 48^{\prime} \mathrm{E}, 31 . \mathrm{V} .2011$, sweeping, Pu Tang,

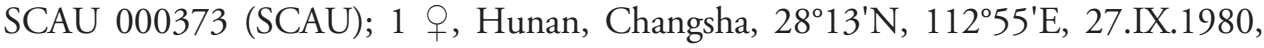
Xinwang Tong, SCAU 000374 (SCAU); 1 , , Guangdong, Chebaling National Nature Reserve, $24^{\circ} 43^{\prime} \mathrm{N}, 114^{\circ} 14^{\prime} \mathrm{E}, 25 . V .2003$, Jingxian Liu, SCAU 000375 (SCAU); 1 ㅇ + $2{ }^{\lambda}$, Guangdong, Chebaling National Nature Reserve, $24^{\circ} 43^{\prime} \mathrm{N}, 114^{\circ} 14^{\prime} \mathrm{E}, 21-23$. VIII.2003, Jingxian Liu, SCAU 000376-000378 (SCAU); 1 ô, Guangdong, Zijin County, Linjiang Town, $23^{\circ} 39^{\prime} \mathrm{N}, 14^{\circ} 41^{\prime} \mathrm{E}, 1$.VIII.2003, Jingxian Liu, SCAU 000379

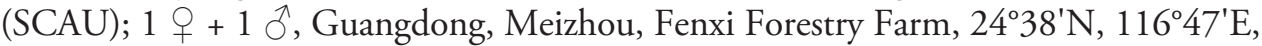


28.VII.2003, Jianuan Zhou, SCAU 000380, 000381 (SCAU); 1 q, Guangdong, Qimuzhang Nature Reserve, $23^{\circ} 46^{\prime} \mathrm{N}, 115^{\circ} 18^{\prime} \mathrm{E}$, 31.VII.2003, Jingxian Liu, SCAU 000382 (SCAU); $1 \uparrow+1 \mathrm{O}^{\top}$, Guangdong, Xinfeng, Mt. Yunji, $24^{\circ} 04^{\prime} \mathrm{N}, 114^{\circ} 10^{\prime} \mathrm{E}$, 19.VII.2003, Yanxia Song, SCAU 000383, 000384 (SCAU); 1 q, Guangdong, Mt. Nankun, $23^{\circ} 37.941$ 'N, 11350.182'E, 14.VII.2003, Jingxian Liu, SCAU 000385 (SCAU); $2{ }^{\top}$, Guangdong, Mt. Nankun, $23^{\circ} 37.941^{\prime} \mathrm{N}, 113^{\circ} 50.182^{\prime} \mathrm{E}, 2$.VII.2005, Zaifu Xu, SCAU 000386, 000387 (SCAU); 2 + , Guangdong, Mt. Nankun, 2337.941'N, $113^{\circ} 50.182^{\prime} \mathrm{E}, 23 . V .2010$, Zaifu Xu, SCAU 000388, 000389 (SCAU); $1+1$ Guangdong, Mt. Nankun, $23^{\circ} 37.941^{\prime} \mathrm{N}, 113^{\circ} 50.182^{\prime} \mathrm{E}, 25-30$.VII.2010, yellow pan trap, Huayan Chen, SCAU 000390, 000391 (SCAU); 1 \%, Guangdong, Mt. Nankun, $23^{\circ} 37.941^{\prime} \mathrm{N}, 113^{\circ} 50.182^{\prime} \mathrm{E}, 540 \mathrm{~m}$, 4.VI.2011, yellow pan trap, Huayan Chen, SCAU 000392 (SCAU); 4 q, Guangdong, Zhaoqing, Xiwanggu, $23^{\circ} 13^{\prime} \mathrm{N}, 112^{\circ} 31^{\prime} \mathrm{E}$, 2-6.VIII.2010, sweeping, Huayan Chen, SCAU 000393-000396 (SCAU); 1 + + 1 ${ }^{\top}$, Guangdong, Zhaoqing, Xiwanggu, $23^{\circ} 13^{\prime} \mathrm{N}, 112^{\circ} 31^{\prime} \mathrm{E}, 2-6 . V I I I .2010$, yellow pan trap, Huayan Chen, SCAU 000397, 000398 (SCAU); 1 \%, Guangdong, Guangzhou, Zengcheng, $23^{\circ} 14^{\prime} \mathrm{N}, 113^{\circ} 38^{\prime} \mathrm{E}, 15 . X I .2010$, Huiting Chen, SCAU 000399 (SCAU); 1 + $+2{ }^{\top}$, Guangdong, Guangzhou, Tianlu Lake, $23^{\circ} 13^{\prime} \mathrm{N}, 113^{\circ} 25^{\prime} \mathrm{E}, 6 . X .2002$, Zaifu Xu, SCAU 000400-000402 (SCAU); $1++1 \widehat{\jmath}$, Guangdong, Guangzhou, Tianlu Lake, $23^{\circ} 13^{\prime} \mathrm{N}, 113^{\circ} 25^{\prime} \mathrm{E}$, 24.VI.2003, Jingxian Liu, SCAU 000403, 000404 (SCAU);

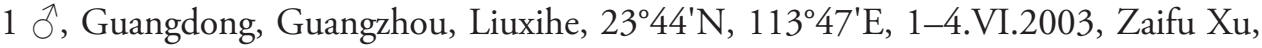

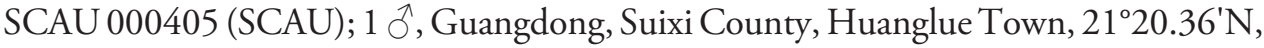
$110^{\circ} 18.61^{\prime} \mathrm{E}, 25 . \mathrm{IX} .2010$, yellow pan trap, Huayan Chen, SCAU 000406 (SCAU); 2 $+1 \partial^{\wedge}$, Hainan, Mt. Yinggeling, $18^{\circ} 49^{\prime} \mathrm{N} 109^{\circ} 11^{\prime} \mathrm{E}, 24-25 . V .2007$, Jingxian Liu, SCAU 000407-000409 (SCAU); 3 9, Hainan, Mt. Yinggeling, 1849' N, $109^{\circ} 11^{\prime} \mathrm{E}$, 22-25.V.2007, Jie Zeng, SCAU 000410-000412 (SCAU); 2 \%, Hainan, Mt. Yinggeling, $18^{\circ} 49^{\prime}$ N $109^{\circ} 11^{\prime} \mathrm{E}, 23-25 . V .2007$, Bin Xiao, SCAU 000413, 000414 (SCAU); 1 O, Hainan, Mt. Yinggeling, $18^{\circ} 49^{\prime} \mathrm{N} 109^{\circ} 11^{\prime} \mathrm{E}, 28 . \mathrm{V} .2007$, Liqiong Weng, SCAU 000415 (SCAU); 1 + , Hainan, Mt. Yinggeling, $18^{\circ} 49^{\prime} \mathrm{N}, 109^{\circ} 11^{\prime} \mathrm{E}, 18 . X .2007$, Jingxian Liu, SCAU 000416 (SCAU); 3 q + 1 ô $^{\prime}$, Hainan, Mt. Yinggeling, $18^{\circ} 49^{\prime} \mathrm{N}, 109^{\circ} 11^{\prime} \mathrm{E}$, 16-20.XI.2008, Huayan Chen, SCAU 000417-000420 (SCAU); 1 ठ̊, Hainan, Mt. Yinggeling, $18^{\circ} 49^{\prime} \mathrm{N}, 109^{\circ} 11^{\prime} \mathrm{E}, 18 . X I .2008$, Jiangli Tang, SCAU 000421 (SCAU); 5

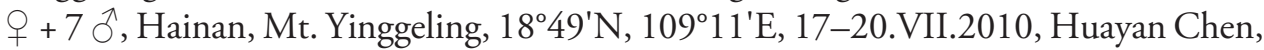
SCAU 000422-000433 (SCAU); 4 q + 5 ${ }^{\lambda}$, Hainan, Baisha, Mt. Jiujialing, 19²14'N, 109²4'E, 18.VII.2010, sweeping, Huayan Chen, SCAU 000434-000442 (SCAU); 2

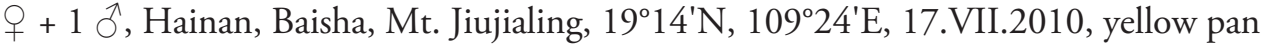
trap, Huayan Chen, SCAU 000443-000445 (SCAU); 8 + 4 ô, Hainan, Wuzhishan National Nature Reserve, $18^{\circ} 51^{\prime} \mathrm{N}, 109^{\circ} 39^{\prime} \mathrm{E}, 16-18 . V .2007$, Jingxian Liu, SCAU 000446-000457 (SCAU); 1 + +2 $\hat{\text {, }}$, Hainan, Wuzhishan National Nature Reserve, $18^{\circ} 51^{\prime} \mathrm{N}, 109^{\circ} 39^{\prime} \mathrm{E}, 16-18 . V .2007$, Jie Zeng, SCAU $000458-000460$ (SCAU); 1 ○े, Hainan, Wuzhishan National Nature Reserve, $18^{\circ} 51^{\prime} \mathrm{N}, 109^{\circ} 39^{\prime} \mathrm{E}, 29 . X .2007$, Jingxian Liu, SCAU 000461 (SCAU); 2 + , Hainan, Wuzhishan National Nature Reserve, $18^{\circ} 51^{\prime} \mathrm{N}, 109^{\circ} 39^{\prime} \mathrm{E}, 28-30 . X .2007$, Jiemin Yao, SCAU 000462, 000463 (SCAU); 1 ${ }^{\top}$, Hainan, Bawangling National Nature Reserve, $19^{\circ} 07^{\prime} \mathrm{N}, 109^{\circ} 03^{\prime} \mathrm{E}$, 9.VI.2007, Jie 


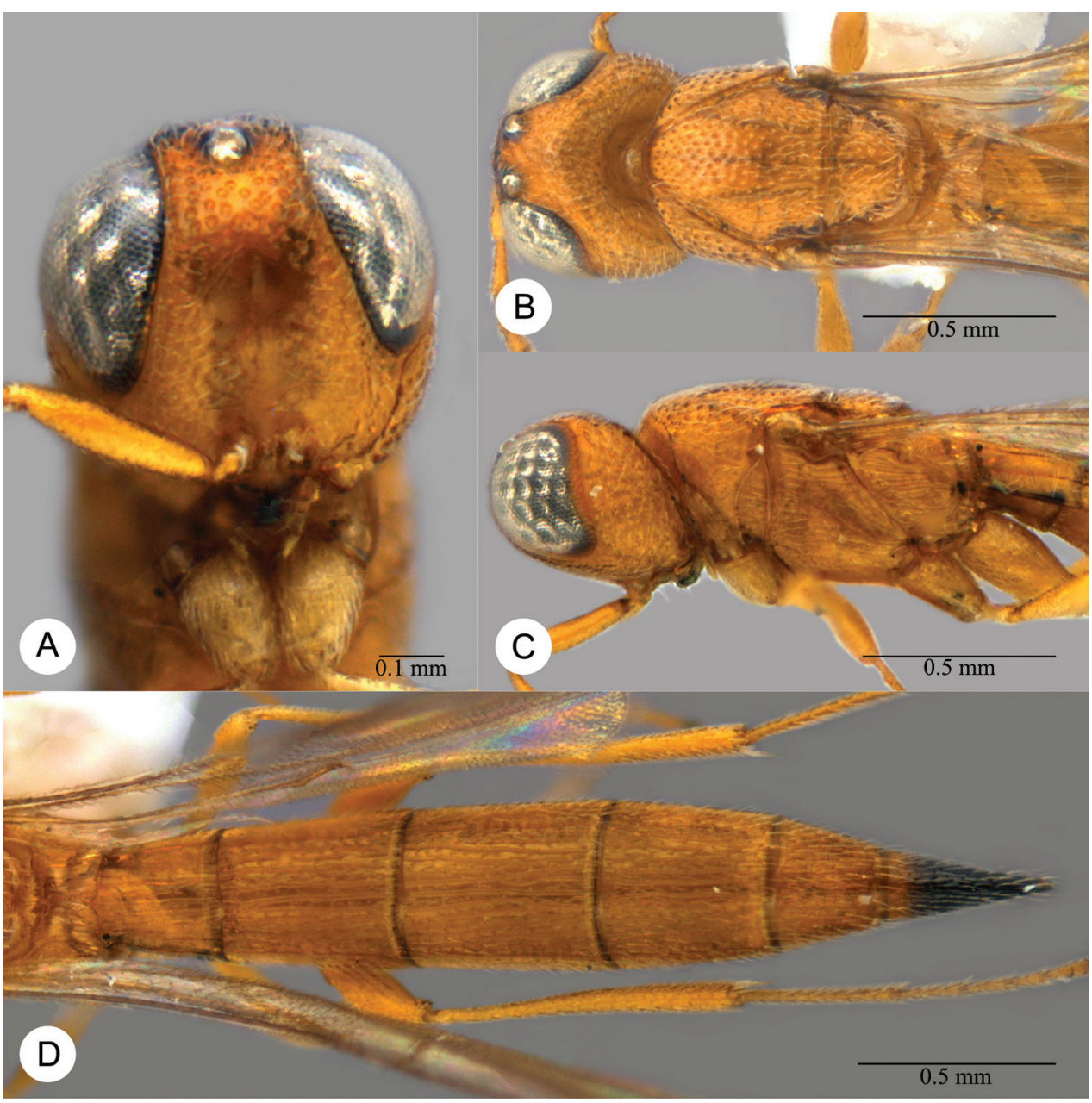

Plate 34. Macroteleia indica Saraswat \& Sharma, female from Hainan, Baisha, Mt. Jiujialing. A Head, anterior view B Head and mesosoma, dorsal view C Head and mesosoma, lateral view D Metasoma, dorsal view.

Zeng, SCAU 000464 (SCAU); 1 §, Hainan, Bawangling National Nature Reserve , $19^{\circ} 07^{\prime} \mathrm{N}, 109^{\circ} 03^{\prime} \mathrm{E}, 1 . \mathrm{V} .2008$, Chundan Hong, SCAU 000465 (SCAU); 2 + , Hainan, Jianfengling National Nature Reserve, $18^{\circ} 41^{\prime} \mathrm{N}, 108^{\circ} 49^{\prime} \mathrm{E}, 4 . V .2008$, Huayan Chen,

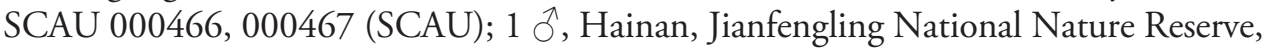
$18^{\circ} 41^{\prime} \mathrm{N}, 108^{\circ} 49^{\prime} \mathrm{E}, 12-14 . \mathrm{VII} .2008$, Jingxian Liu, SCAU 000468 (SCAU); 1 , Hainan, Mt. Diaoluo, 18³9'N, 10953'E, 28.V-1.VI.2007, Jie Zeng, SCAU 000469

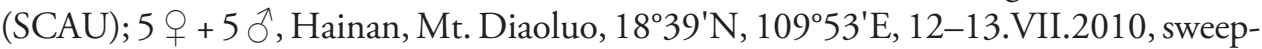
ing, Huayan Chen, SCAU 000470-000479 (SCAU); 1 ㅇ, Hainan, Sanya, Yacheng County, $18^{\circ} 22^{\prime} \mathrm{N}, 109^{\circ} 10^{\prime} \mathrm{E}, 21 . X I .2008$, Manman Wang, SCAU 000480 (SCAU); $1 \delta^{\lambda}$, Guangxi, Longshan Nature Reserve, 2324.73'N, 108 31.92'E, 370m, 1.VII-2. VII.2011, yellow pan trap, Zaifu Xu, SCAU 000481 (SCAU); 2 §, Guangxi, Longshan 


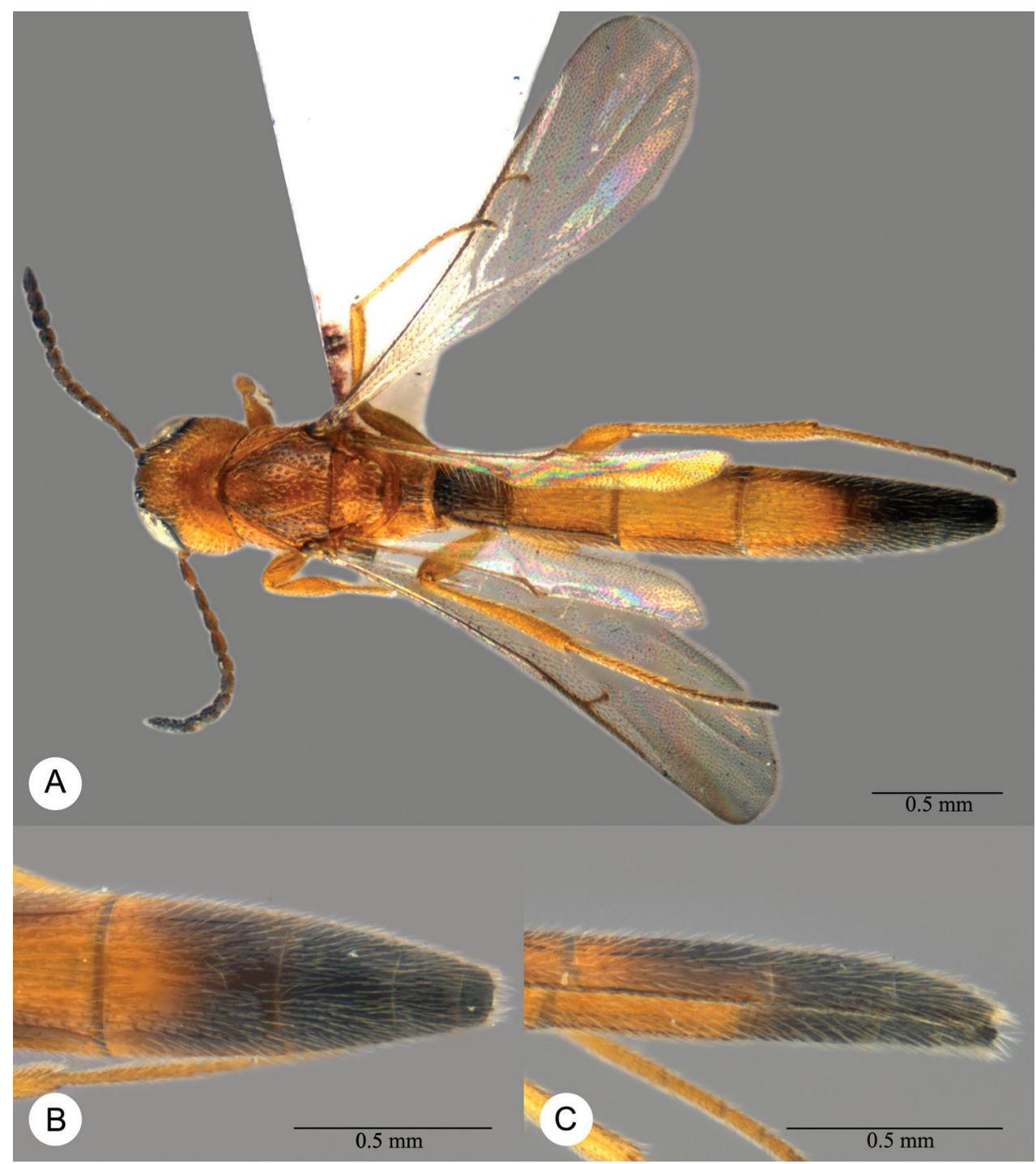

Plate 35. Macroteleia indica Saraswat \& Sharma, male from Hainan, Baisha, Mt. Jiujialing. A Dorsal habitus B Apex of metasoma, dorsal view C Apex of metasoma, lateral view.

Nature Reserve, $23^{\circ} 24.73^{\prime} \mathrm{N}, 108^{\circ} 31.92^{\prime} \mathrm{E}, 370 \mathrm{~m}, 1-2 . \mathrm{VII} .2011$, sweeping, Huiting Chen, SCAU 000482, 000483 (SCAU); 3 q +2 ふै, Yunnan, Gejiu, Manhao Town, $23^{\circ} 01^{\prime} \mathrm{N}, 103^{\circ} 20^{\prime} \mathrm{E}, 23$.VII.2003, Jingxian Liu, SCAU 000484-000488 (SCAU); 1 ㅇ, Yunnan, Dehong County, Nabang Town, 24²4'N, 97³4'E, 15.V.2009, Manman Wang, SCAU 000489 (SCAU); 2 ㅇ, Yunnan, Xishuangbanna Primeval Forest Park, $22^{\circ} 01^{\prime} \mathrm{N}, 100^{\circ} 52^{\prime} \mathrm{E}, 30 . \mathrm{VII} .2003$, Long Hu, SCAU 000490, 000491 (SCAU); 2 + + 2 đ., Yunnan, Hekou County, Nanxi Town, $22^{\circ} 37^{\prime}$ N, $103^{\circ} 56^{\prime} \mathrm{E}, 20 . \mathrm{VII} .2003$, Long Hu, SCAU 000492-000495 (SCAU). 


\section{Macroteleia lamba Saraswat \& Sharma}

http://species-id.net/wiki/Macroteleia_lamba

Plates 36-41

Macroteleia lamba Saraswat and Sharma 1978: 13 (original description); Mani and Sharma 1982: 172 (description); Mukerjee 1994: 4 (variation); Lê 2000: 53, 61 (description, keyed, type information); Rajmohana 2006: 127 (description); Rajmohana 2007: 64 (description).

Macroteleia dores Lê 2000: 53, 58, 333 (original description, keyed), syn. n.

Description. Female. Body length 3.33-5.26 mm ( $\mathrm{n}=18)$.

Color. Body black; mandible dark brown; palpi yellow; legs pale brown throughout; A1 brown, A2-A5 dark brown, remainder of antenna black; fore wing hyaline.

Head. Transverse in dorsal view, 1.24-1.36x as wide as long, slightly wider than mesosoma; lateral ocellus contiguous with inner orbit of compound eye; POL 1.311.58x LOL; occipital carina continuous medially, irregularly crenulate throughout; central keel absent or weakly developed above interantennal process (Plates 37A, 38C, 40A); medial frons obliquely strigose ventrally, irregularly smooth dorsally; ventrolateral frons punctate rugose; frons below median ocellus densely punctate, interspaces coriaceous; ocellar triangle coriaceous, with scattered punctures; posterior vertex punctate reticulate; gena coarsely punctate rugose; length of A3 equal to length of A2.

Mesosoma. Cervical pronotal area densely punctate; dorsal pronotal area areolate; lateral pronotal area rugulose anteriorly, punctate rugulose posteriorly; netrion punctate rugulose; notaulus deep, distinctly foveolate; middle lobe of mesoscutum densely punctate, becoming denser anteriorly and at posterior end; lateral lobes of mesoscutum densely punctate, interspaces in part coriaceous; mesoscutellum densely punctate throughout; Metascutellum triangular (Plates 37B, 38D, 40B), strongly produced medially, extending into space between propodeal lobes; propodeum narrowly divided into two subtriangular lobes (Plates 37B, 38D, 40B), each side with several irregular longitudinal carinae medially, otherwise punctate rugulose; upper mesepisternum with a row of robust longitudinal carinae below subalar pit; lower mesepisternum punctate rugulose; mesopleural depression smooth (Plates 37C, 38E, 40C); metapleuron longitudinally striate throughout.

Legs. Slender; hind femur weakly swollen, $4.00-5.00 \times$ as long as its maximum width; hind tibia without spines over outer surface; hind basitarsus $11.00-11.80 \times$ as long as its maximum width.

Wings. Apex of fore wing extending from as far as anterior third to posterior margin of T4; R 1.62-2.44x as long as r-rs, R1 1.91-2.24x length of R.

Metasoma. Posterior margin of transverse sulcus on T2 straight or slightly convex (Plates 37D, 38F, 40D); sublateral tergal carinae developed on T1-T3; T1-T3 densely longitudinally striate medially, with scattered delicate punctures in interstices, punctate rugulose laterally; T4-T5 densely longitudinally striate throughout, with delicate punctures in interstices; T6 finely punctate dorsally, densely longitudinally striate laterally, with scattered small punctures in interstices; length of T3 $0.91-1.15 \times$ length 


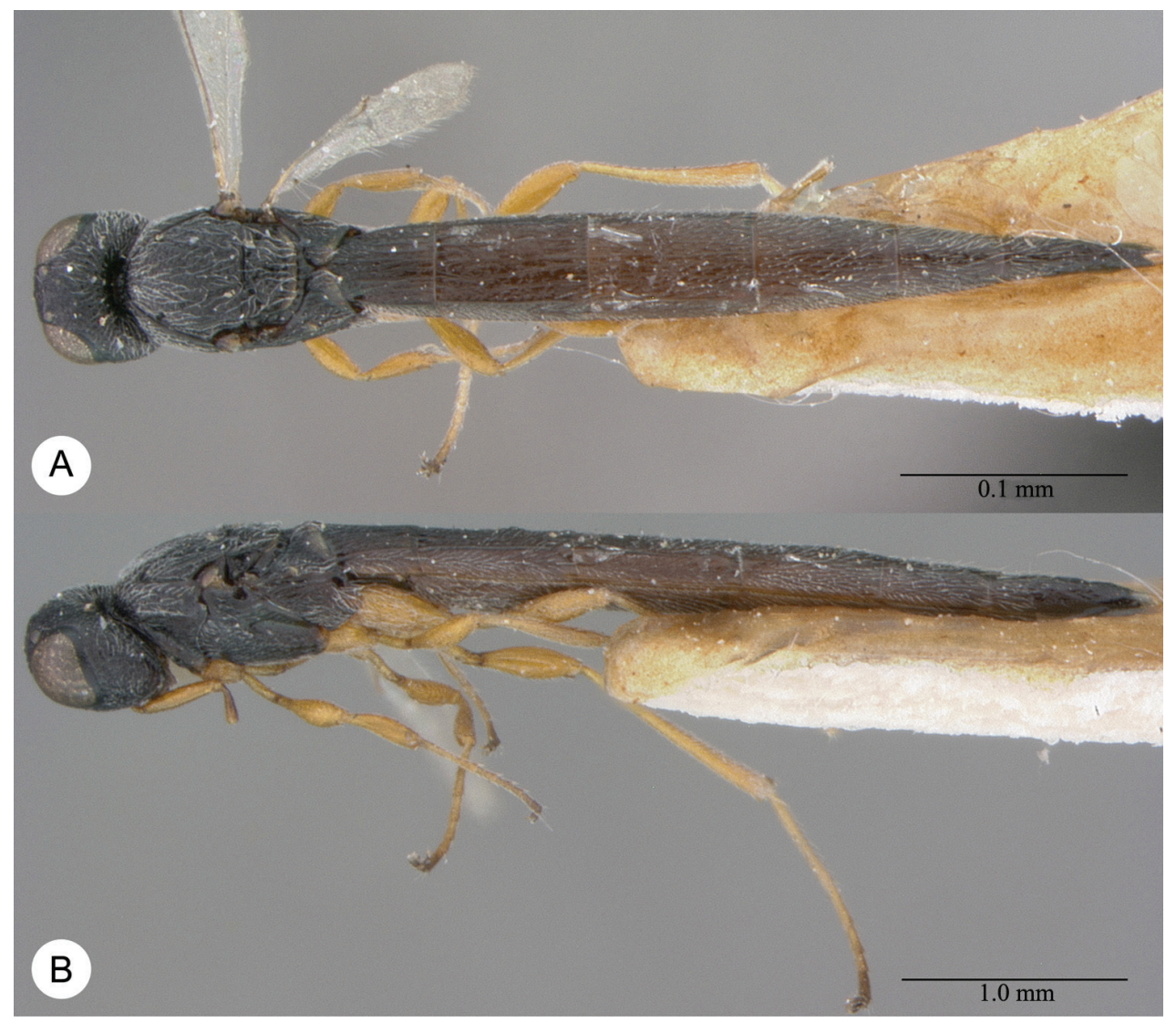

Plate 36. Macroteleia lamba Saraswat \& Sharma, holotype, female. A Dorsal habitus B Lateral habitus.

of T6; T5 distinctly longer than wide; S2-S6 densely longitudinally striate, with delicate punctures in interstices; prominent longitudinal median carina present on S2-S5. Male. Differing from female as follows: body length 3.57-5.26 mm ( $=6)$; A1 brown, remainder of antenna dark brown to black; hind coxa blackish; metascutellum distinctly transverse (Plate 41A), posterior margin slightly pointed medially, longitudinally carinate; propodeum continuous medially, not divided into two separated lobes, posterior margin narrowly notched medially, each side with several irregular longitudinal carinae medially, otherwise punctate rugulose, covered by dense, recumbent, white setae; T1 sparsely longitudinally striate medially, with rugulose sculpture in interstices anteriorly, punctate rugulose laterally; T2-T3 densely longitudinally striate medially, with numerous delicate punctures in interstices, punctate rugulose laterally; T4-T5 densely longitudinally striate throughout, with numerous delicate punctures in interstices; T6-T7 longitudinally punctate rugulose; T6 distinctly longer than wide; length of T6 1.07-1.18x length of T7; T7 subtriangular, apex pointed (Plate 41B); length of T7 2.15-2.53× length of S7; S7 longitudinally punctate rugulose; prominent longitudinal median carina present on S2-S6. 


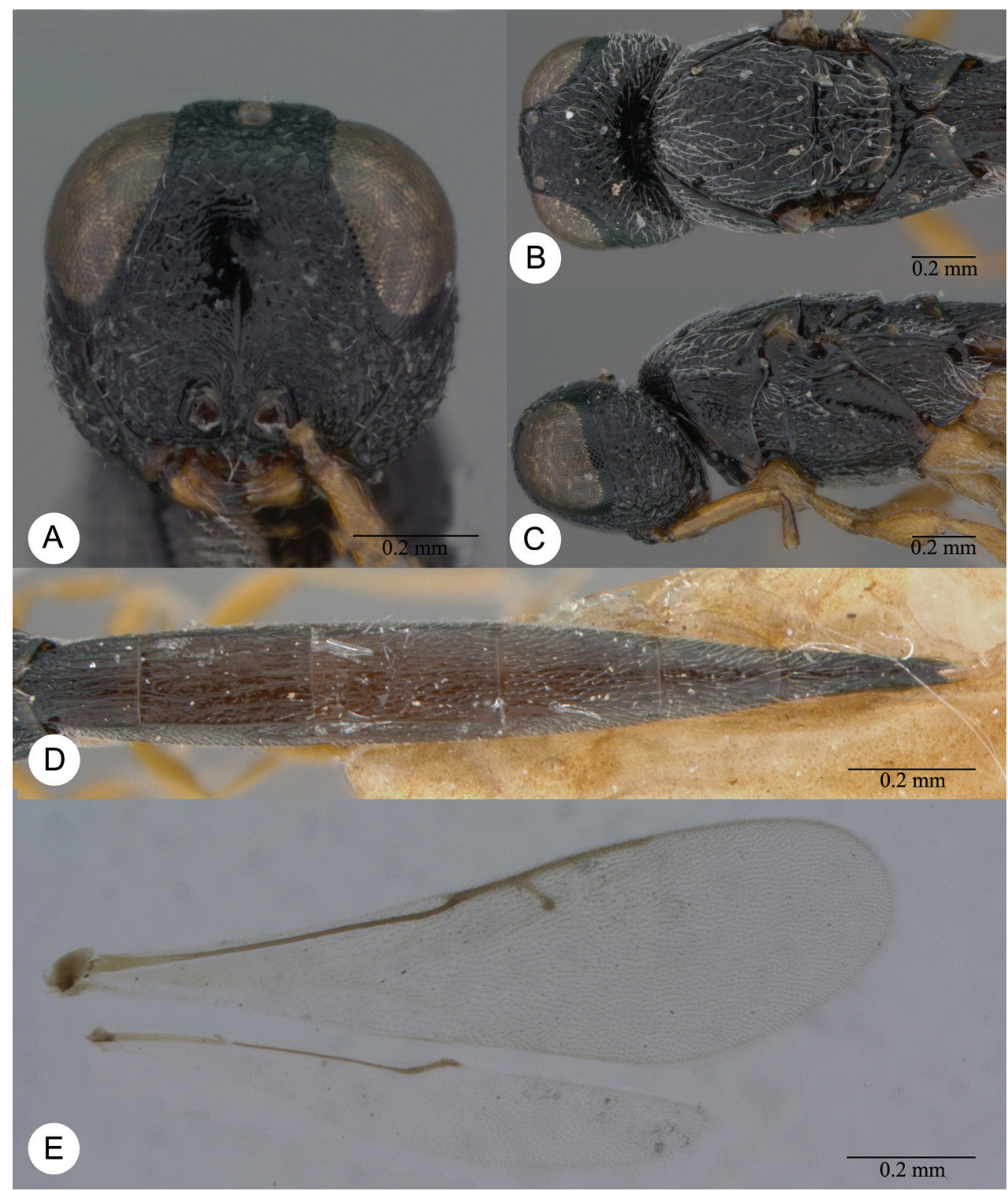

Plate 37. Macroteleia lamba Saraswat \& Sharma, holotype, female. A Head, anterior view B Head and mesosoma, dorsal view $\mathbf{C}$ Head and mesosoma, lateral view D Metasoma, dorsal view E Fore and hind wing.

Distribution. China (Guangdong, Hainan, Yunnan); India; Vietnam; Thailand. Link to distribution map [http://hol.osu.edu/map-large.html?id=4825].

Material examined. Holotype of M. lamba, o+: INDIA: "Coorg Survey, School of Entomology, St. John’s College, Agra-282002, India, 18.1. Kasaragod, M.S. Mani \& Party, 31.III-1-3.IV.1977”, "Holotype", "Macroteleia lamba Saraswat, \$” (deposited in USNM). Holotype of M. dores, \$: VIETNAM: "Buon Luoi, grass, 16.VI.1982", 


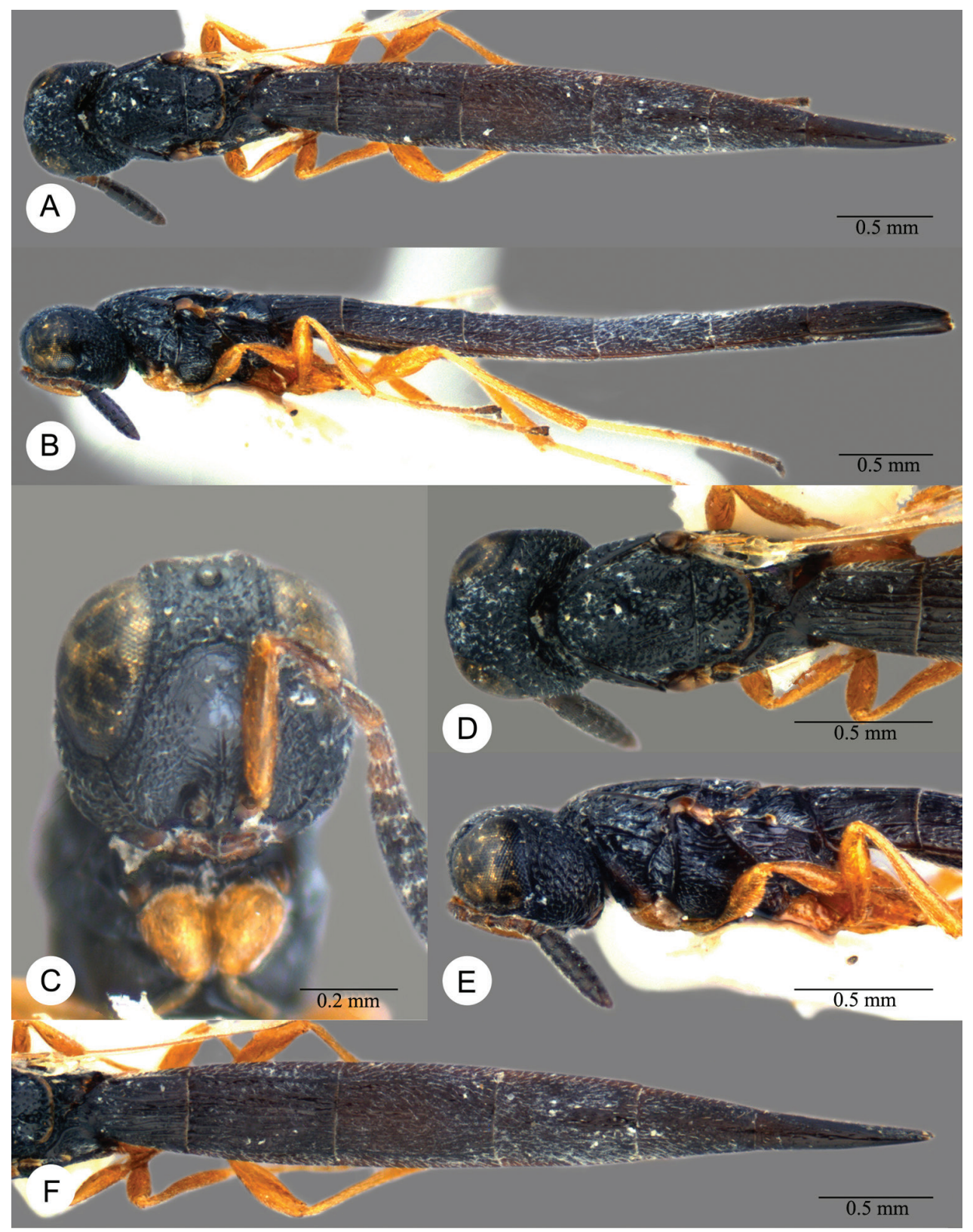

Plate 38. Macroteleia dores Kozlov \& Lê, holotype, female. A Dorsal habitus B Lateral habitus C Head, anterior view D Head and mesosoma, dorsal view E Head and mesosoma, lateral view F Metasoma, dorsal view.

"Holotypus 9 Macroteleia dores Kozlov et Lê 84" (deposited in IEBR). Paratype of $M$. dores, 1 त, VIETNAM: "Buon Luoi, GL-KT [=Gia Lai-Kon Tum], grass, 14.VII.1981", "Paratypus ổ Macroteleia dores sp. n. Kozlov et Lê" (IEBR). 


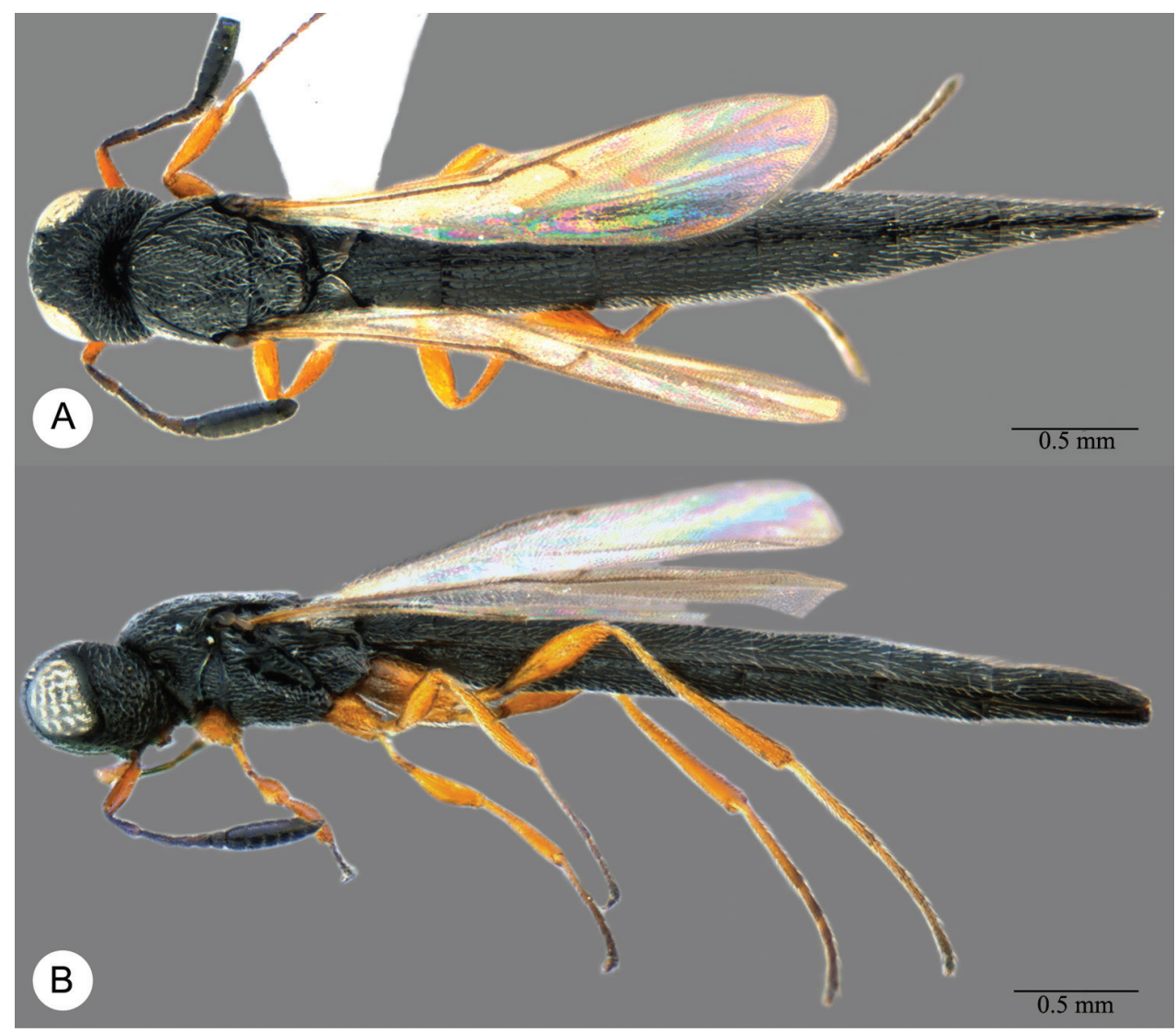

Plate 39. Macroteleia lamba Saraswat \& Sharma, female from Yunnan, Jinggu County, Weiyuan Town. A Dorsal habitus B Lateral habitus.

Other material. CHINA: $1 \hat{\jmath}$, Guangdong, Chebaling National Nature Reserve, $24^{\circ} 43^{\prime} \mathrm{N}, 114^{\circ} 14^{\prime} \mathrm{E}, 10 . \mathrm{VII} .2003$, Zaifu Xu, SCAU 000118 (SCAU); 4 q, Guangdong, Chebaling National Nature Reserve, $24^{\circ} 43^{\prime} \mathrm{N}, 114^{\circ} 14^{\prime} \mathrm{E}, 21-23$.VIII.2003, Jingxian Liu, SCAU 000119-000122 (SCAU); 1 +, Guangdong, Nanling National Nature Reserve, $24^{\circ} 54^{\prime} \mathrm{N}, 113^{\circ} 00^{\prime} \mathrm{E}, 11 . \mathrm{IX} .2004$, Yanjing Wen, SCAU 000123 (SCAU); 1 ㅇ, Guangdong, Qimuzhang Nature Reserve, $23^{\circ} 46^{\prime} \mathrm{N}, 115^{\circ} 18^{\prime} \mathrm{E}, 31$.VII.2003, Jingxian Liu, SCAU 000124 (SCAU); $10^{\lambda}$, Guangdong, Zijin County, Linjiang Town, 2339' N, 114²41'E, 28.VII.2003, Jingxian Liu, SCAU 000125 (SCAU); 3 + + $3 \hat{\gamma}$, Guangdong, Zijin County, Linjiang Town, $23^{\circ} 39^{\prime} \mathrm{N}, 114^{\circ} 41^{\prime} \mathrm{E}, 1$.VIII.2003, Jingxian Liu, SCAU 000126-000131 (SCAU); 1 ㅇ, Guangdong, paddy field, Zhuhai, Doumen, $22^{\circ} 33.366^{\prime} \mathrm{N}, 113^{\circ} 13.297^{\prime} \mathrm{E}$, 11.IX.2010, Xin Yuan, SCAU 000132 (SCAU); 1 +, Hainan, Mt. Yinggeling, 1849'N, $109^{\circ} 11^{\prime} E$, 28.V.2007, Liqiong Weng, SCAU 000133 (SCAU); 1 ㅇ, Hainan, Jianfengling National Nature Reserve, $18^{\circ} 41^{\prime} \mathrm{N}, 108^{\circ} 49^{\prime} \mathrm{E}, 22-23 . X .2007$, Jingxian Liu, SCAU 000134

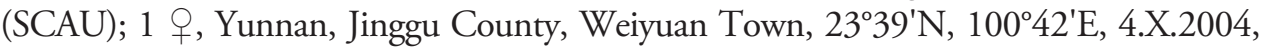
Jingxian Liu, SCAU 000135 (SCAU); 3 \&, Yunnan, Jinghong, on the bank of Lancang 


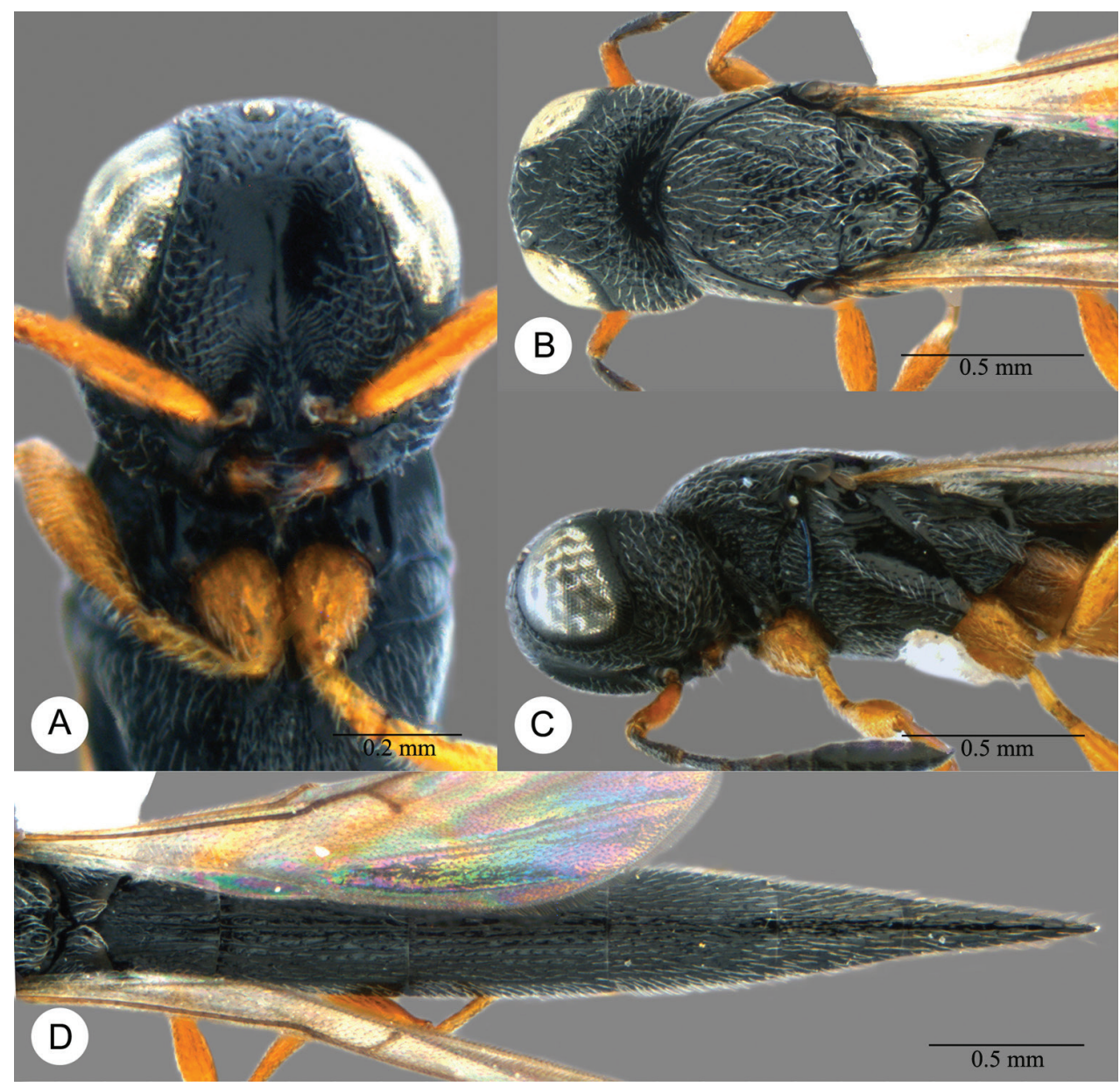

Plate 40. Macroteleia lamba Saraswat \& Sharma, female from Yunnan, Jinggu County, Weiyuan Town. A Head, anterior view B Head and mesosoma, dorsal view C Head and mesosoma, lateral view D Metasoma, dorsal view.

River, 2159'N, 10049'E, 2.X.2004, Jingxian Liu, SCAU 000136-000138 (SCAU). THAILAND: 1 q, Chiang Mai: Maerim, 21-22.XII.2000, MT, R. A. Beaver, No. 24062 (RABC); 1 ㅇ, Chiang Mai: Maerim, 20.XII.2002, FIT, R. A. Beaver, No. 25927 (RABC).

Comments. Macroteleia superans Kieffer was described from a male collected at Mt. Makiling in the Philippine Islands by C.F. Baker. Later, in Kieffer's Das Tierreich (Kieffer 1926) treatment of the species he added the locality of Mt. Banahao for the species. We searched for the type material at the U.S. National Museum of Natural History (Washington, DC) where much of Baker's material is now held. Only a single specimen of $M$. superans was found, the specimen from Mt. Banahao. The location of the holotype is unknown. This non-type specimen is conspecific with M. lamba. Without the holotype, though, we prefer not to propose that the name is synonymous. 


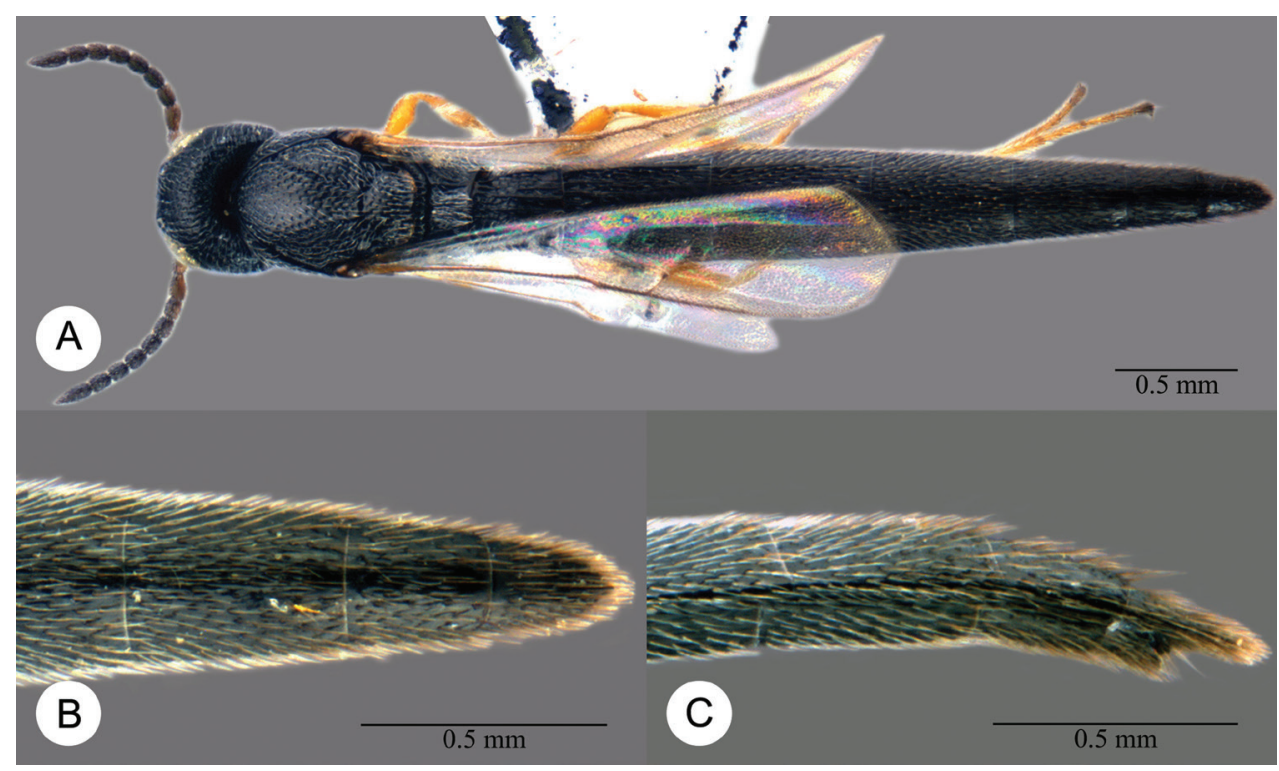

Plate 4I. Macroteleia lamba Saraswat \& Sharma, male from Guangdong, Zijin County, Linjiang Town. A Dorsal habitus B Apex of metasoma, dorsal view C Apex of metasoma, lateral view.

\section{Macroteleia livingstoni Saraswat}

http://species-id.net/wiki/Macroteleia_livingstoni

Plates 42-46

Macroteleia livingstoni Saraswat 1982: 348 (original description).

Description. Female. Body length 3.28-4.08 mm ( $\mathrm{n}=20)$.

Color. Body black; mandible dark brown; palpi light brown; legs pale brown throughout; A1 pale brown, A2-A6 brown, remainder of antenna black; fore wing hyaline.

Head. Transverse in dorsal view, 1.20-1.34x as wide as long, slightly wider than mesosoma; lateral ocellus contiguous with inner orbit of compound eye; POL 1.31$1.45 \times$ LOL; occipital carina continuous medially; central keel weakly developed above interantennal process (Plates 43A, 45A); medial frons obliquely strigose ventrally, irregularly smooth dorsally; ventrolateral frons punctate rugose; frons below median ocellus sparsely punctate; ocellar triangle smooth, with scattered punctures; posterior vertex densely punctate; gena punctate reticulate; length of A3 0.83-1.00× length of A2.

Mesosoma. Cervical pronotal area densely punctate; dorsal pronotal area densely coarsely punctate; lateral pronotal area smooth in upper anterior angle, punctate rugose posteriorly; netrion punctate rugulose; notaulus narrow, but distinctly foveolate; middle lobe of mesoscutum densely punctate anteriorly and at posterior end, usually sparsely punctate on a small area across middle; lateral lobe of mesoscutum densely punctate throughout; mesoscutellum densely punctate throughout; metascutellum transverse (Plates 43B, 45B), posterior margin slightly pointed medially, longitudinal- 


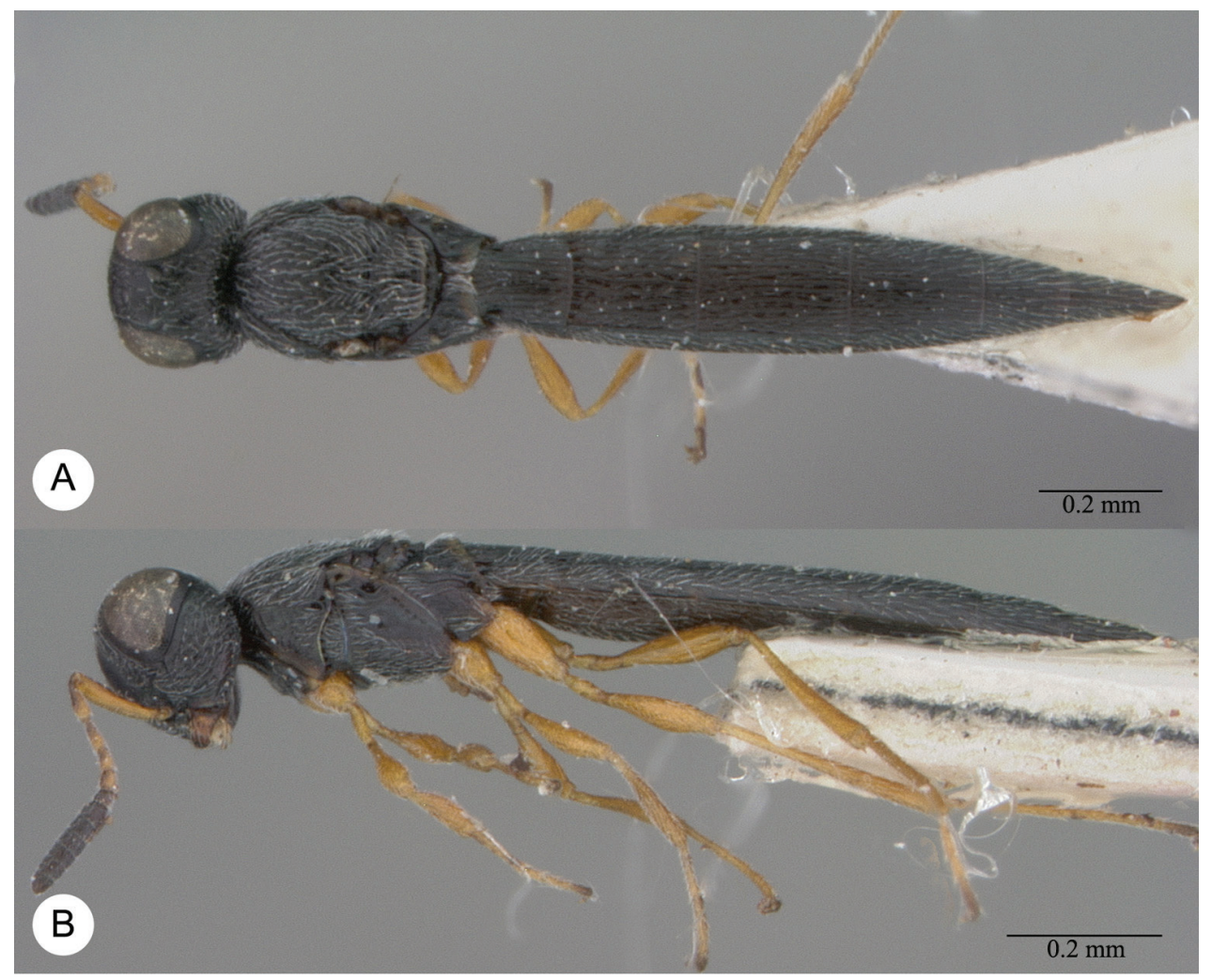

Plate 42. Macroteleia livingstoni Saraswat, holotype, female. A Dorsal habitus B Lateral habitus.

ly carinate; propodeum continuous medially (Plates 43B, 45B), not divided into two separated lobes, posterior margin narrowly notched medially, each side with several irregular longitudinal carinae medially, otherwise punctate rugulose, covered by dense, recumbent, white setae; upper mesepisternum with a row of weak longitudinal carinae or a ledge below subalar pit; lower mesepisternum variably smooth to punctate rugulose; mesopleural depression smooth (Plates 43C, 45C); metapleuron longitudinally striate dorsally, punctate rugulose ventrally.

Legs. Slender; hind femur weakly swollen, 3.86-4.69x as long as its maximum width; hind tibia without spines over outer surface; hind basitarsus $10.50-13.00 \times$ as long as its maximum width.

Wings. Apex of fore wing extending from as far as posterior fifth to posterior margin of T4; R 1.82-2.18x as long as r-rs, R1 1.65-2.18× length of R.

Metasoma. Posterior margin of transverse sulcus on T2 slightly convex (Plates 43D, 45D); sublateral tergal carinae developed on T1-T3; T1-T3 densely longitudinally striate medially, with scattered delicate punctures in interstices, punctate rugulose laterally; T4-T5 densely longitudinally striate throughout, with delicate punctures in interstices; T6 finely punctate dorsally, densely longitudinally striate laterally, with scattered small punctures in interstices; length of T3 1.00-1.13× length of T6; T5 


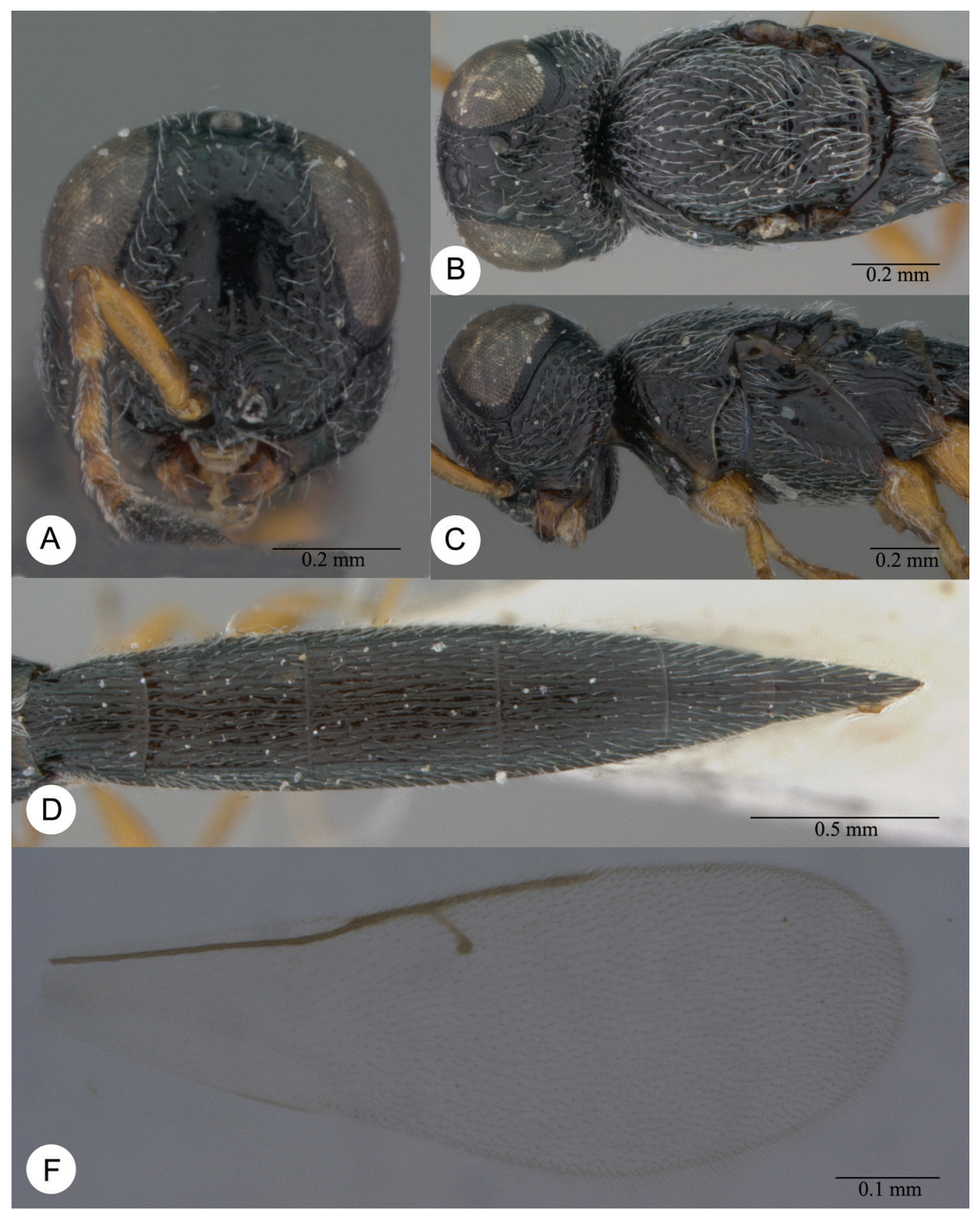

Plate 43. Macroteleia livingstoni Saraswat, holotype, female. A Head, anterior view B Head and mesosoma, dorsal view C Head and mesosoma, lateral view D Metasoma, dorsal view E Fore wing.

slightly longer than wide; S2-S6 densely longitudinally striate, with delicate punctures in interstices; prominent longitudinal median carina present on S2-S5.

Male. Differing from female as follows: body length 3.29-3.92 mm ( $\mathrm{n}=20)$; A1 pale brown, remainder of antenna dark brown, becoming darker apically; T5-T6 


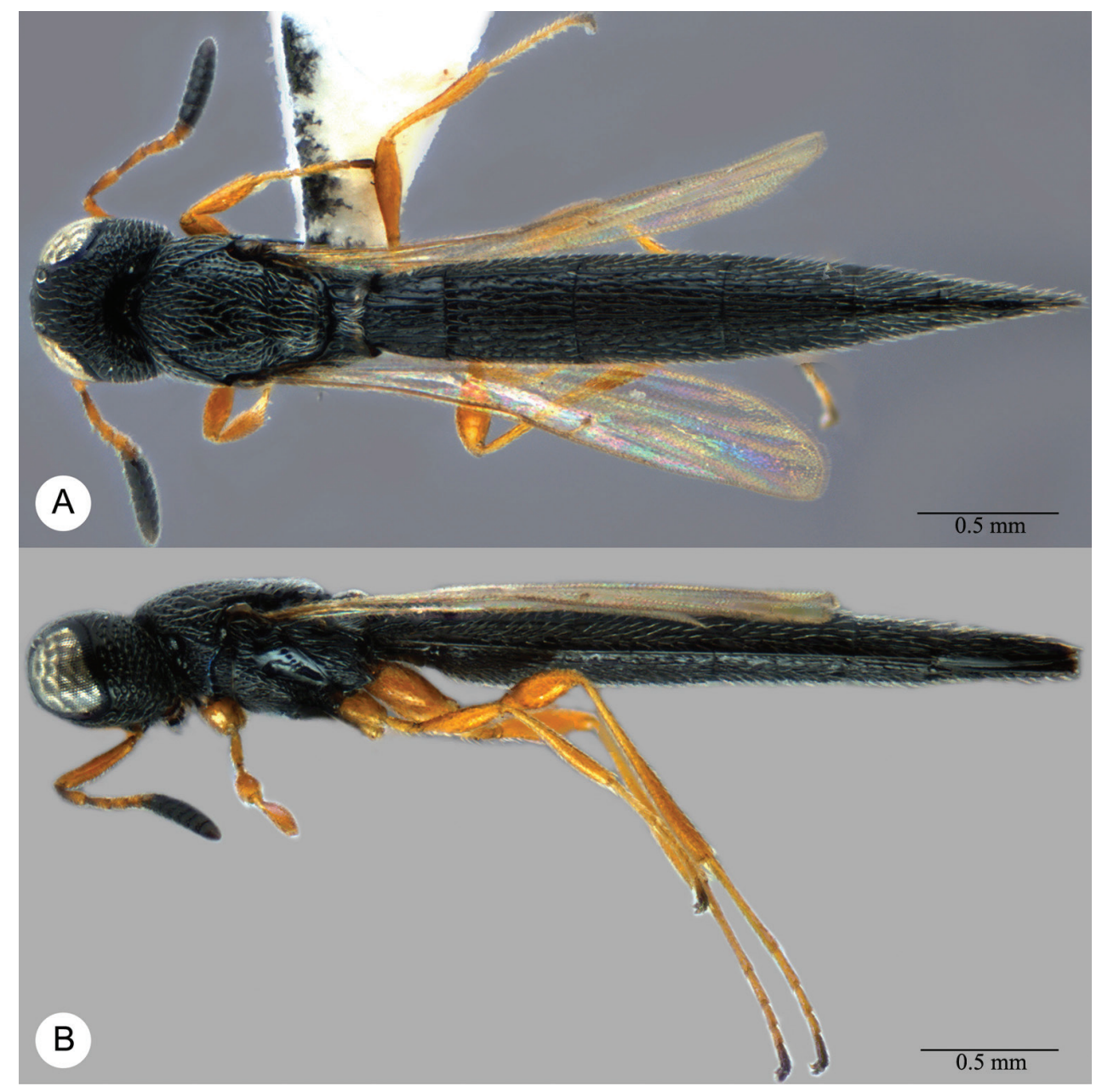

Plate 44. Macroteleia livingstoni Saraswat, female from Guangdong, Zhaoqing, Xiwanggu. A Dorsal habitus B Lateral habitus.

moderately longitudinally striate, with delicate punctures in interstices; T7 punctate rugulose throughout; T6 distinctly longer than wide; length of T6 1.00-1.19× length of T7; T7 subtriangular, apex pointed (Plate 46B); length of T7 1.56-2.40× length of S7; S6-S7 longitudinally punctate rugulose; prominent longitudinal median carina present on S2-S6.

Diagnosis. M. livingstoni is similar to M. emarginata in body shape and color, but can be distinguished by its smller size and reticulate gena. The male of $M$. livingstoni is also similar to that of $M$. lamba, but can be distinguished by the fact that the frons below the median ocellus is sparsely punctate with the interspaces smooth (densely punctate, interspaces coriaceous in $M$. lamba); ocellar triangle smooth, with scattered punctures (coriaceous, with scattered punctures in M. lamba). 


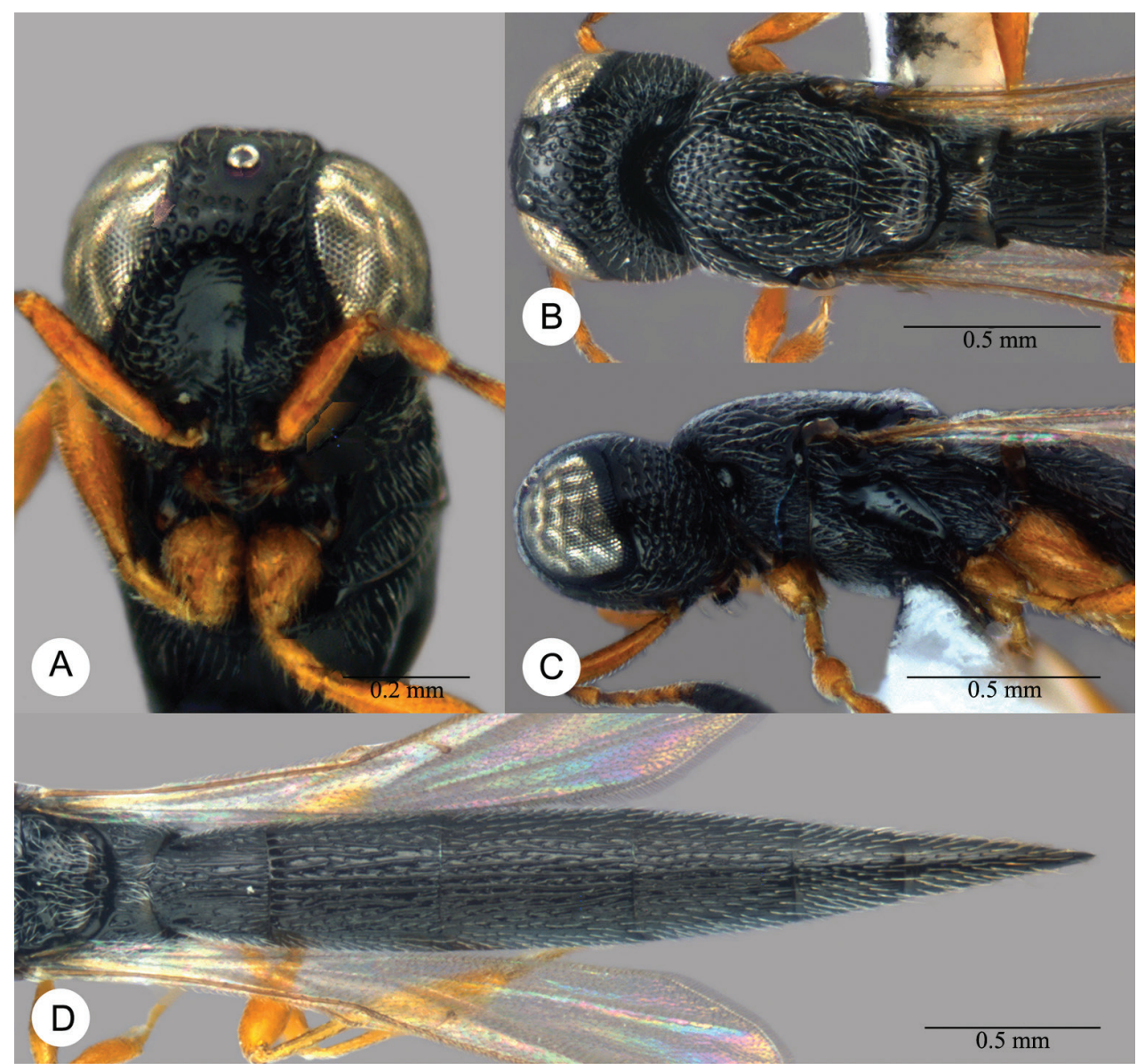

Plate 45. Macroteleia livingstoni Saraswat, female from Guangdong, Zhaoqing, Xiwanggu. A Head, anterior view B Head and mesosoma, dorsal view C Head and mesosoma, lateral view D Metasoma, dorsal view.

Distribution. China (Hubei, Guangdong, Hainan, Guizhou, Guangxi, Yunnan); India. Link to distribution map [http://hol.osu.edu/map-large.html?id=4831].

Material examined. Holotype, + : INDIA: "Karnataka Survey, School of Entomology, St. John’s College, Agra-282002, India, 20.6.Walayar, M.S. Mani \& Party, 9-11.V.1978", "Holotype", "Macroteleia livingstoni Saraswat, \$” (deposited in USNM).

Other material. CHINA: 1 q Guangdong, Nanling National Nature Reserve, $24^{\circ} 54^{\prime} \mathrm{N}, 113^{\circ} 00^{\prime} \mathrm{E}, 8-17 . V I I I .2010$, Huayan Chen, SCAU 000189; 3 q, Hubei, Huanggang, Mt. Dahu, 31 $27^{\prime} \mathrm{N}, 114^{\circ} 32^{\prime} \mathrm{E}$, VIII.2009, Chunhong Zheng, SCAU 000497-000499 (SCAU); 2 + 4 ô, Guangdong, Chebaling National Nature Reserve, $24^{\circ} 43^{\prime} \mathrm{N}, 114^{\circ} 14^{\prime} \mathrm{E}, 21-23 . V I I I .2003$, Jingxian Liu, SCAU 000190-000195

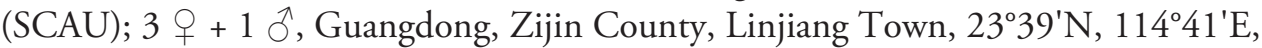
1.VIII.2003, Jingxian Liu, SCAU 000196-000199 (SCAU); 7 + +2 ${ }^{2}$, Guangdong,

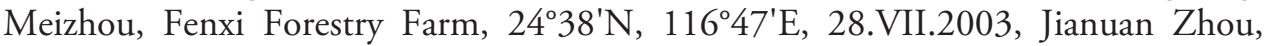




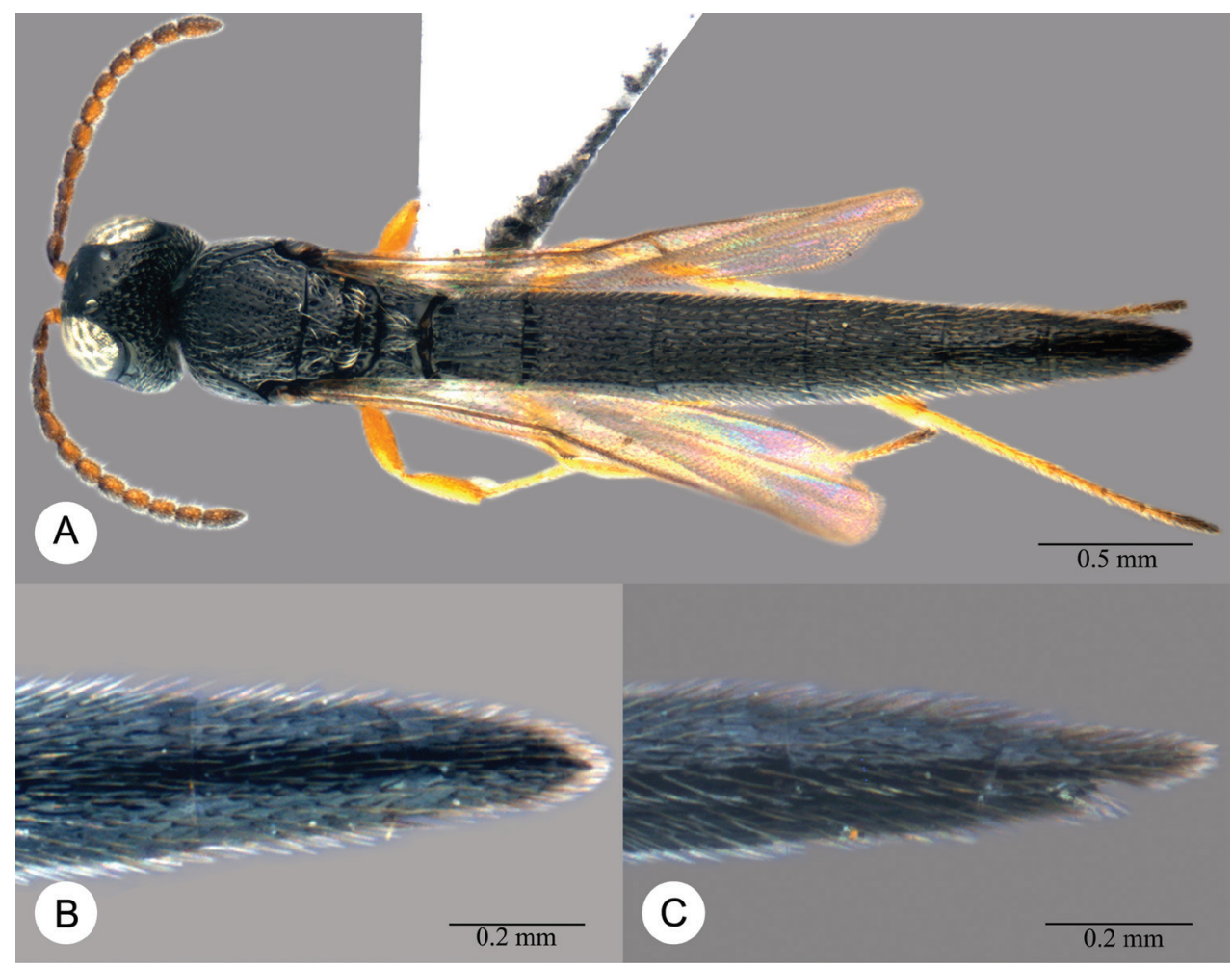

Plate 46. Macroteleia livingstoni Saraswat, male from Hainan, Baisha, Mt. Jiujialing. A Dorsal habitus B Apex of metasoma, dorsal view C Apex of metasoma, lateral view.

SCAU 000200-000208 (SCAU); 1 + , Guangdong, Qimuzhang Nature Reserve, $23^{\circ} 46^{\prime} \mathrm{N}, 115^{\circ} 18^{\prime} \mathrm{E}, 31 . \mathrm{VII} .2003$, Jingxian Liu, SCAU 000209 (SCAU); 1 ㅇ, Guangdong, Boluo County, Yangcun Town, 2325'N, 114²9'E, VIII. 2007, SCAU 000210 (SCAU); $48++1 \partial^{\top}$, Guangdong, Zhaoqing, Xiwanggu, $23^{\circ} 13^{\prime} \mathrm{N}, 112^{\circ} 31^{\prime} \mathrm{E}, 2-6$. VIII.2010, sweeping, Huayan Chen, SCAU 000211-000259 (SCAU); 1 q + 4 ô, Guangdong, Guangzhou, Tianlu Lake, $23^{\circ} 13^{\prime} \mathrm{N}, 113^{\circ} 25^{\prime} \mathrm{E}$, 24.VI.2003, Jingxian Liu, SCAU 000260-000264 (SCAU); 6 + +2 ô, Hainan, Mt. Yinggeling, 1849'N, $109^{\circ} 11^{\prime} \mathrm{E}, 18 . X .2007$, Jingxian Liu, SCAU 000265-000272 (SCAU); 2 9, Hainan, Mt. Yinggeling, $18^{\circ} 49^{\prime} \mathrm{N}, 109^{\circ} 11^{\prime} \mathrm{E}, 16-20 . \mathrm{XI} .2008$, Huayan Chen, SCAU 000273,

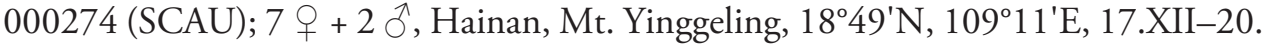
XII.2010, Huayan Chen, SCAU 000275-000283 (SCAU); 10 q + 11 §, Hainan, Baisha, Mt. Jiujialing, $19^{\circ} 14^{\prime} \mathrm{N}, 109^{\circ} 24^{\prime} \mathrm{E}, 18 . \mathrm{VII} .2010$, sweeping, Huayan Chen, SCAU 000284-000304 (SCAU); 2 ㅇ, Hainan, Mt. Diaoluo, 18³9'N, 109 $53^{\circ} \mathrm{E}$, 29.V.2007, Bin Xiao, SCAU 000305, 000306 (SCAU); 1 + , Hainan, Mt. Diaoluo, 12-13.VII.2010, $18^{\circ} 39^{\prime} \mathrm{N}, 109^{\circ} 53^{\prime} \mathrm{E}$, Huayan Chen, SCAU 000307 (SCAU); 1 우 $+1 \delta^{\lambda}$, Guizhou, Guiyang Forest Park, 26 33'N, 106 $44^{\prime} \mathrm{E}$, 23.IX.2007, Cuihong Xie, SCAU 000308, 000309 (SCAU); $1+1$ +1 , Guangxi, Longshan Nature Re- 
serve, $23^{\circ} 24.73^{\prime} \mathrm{N}, 108^{\circ} 31.92^{\prime} \mathrm{E}, 370 \mathrm{~m}, 1-2 . \mathrm{VII} .2011$, yellow pan trap, Zaifu Xu, SCAU 000310, 000311 (SCAU); 4 + +6 , Guangxi, Longshan Nature Reserve, $23^{\circ} 24.73^{\prime} \mathrm{N}, 108^{\circ} 31.92^{\prime} \mathrm{E}, 1-2$.VII.2011, sweeping, Huayan Chen, SCAU 000312 000321 (SCAU); $1 \hat{O}^{\lambda}$, Guangxi, Shanlin, Longshan Nature Reserve, $23^{\circ} 24.73^{\prime} \mathrm{N}$, 108 31.92'E, 1-2.VII.2011, sweeping, Huiting Chen, SCAU 000322 (SCAU); 1 $\widehat{\partial}$, Yunnan, Yingjiang, Tongbiguan, $24^{\circ} 15^{\prime} \mathrm{N}, 97^{\circ} 49^{\prime} \mathrm{E}, 16 . \mathrm{V} .2009$, Manman Wang, SCAU 000323 (SCAU).

\section{Macroteleia peliades Kozlov \& Lê}

http://species-id.net/wiki/Macroteleia_peliades

Plates $47-50$

Macroteleia peliades Lê 2000: 54, 62, 335 (original description, keyed).

Description. Female. Body length 7.28-8.50 mm (n=5).

Color. Body black; mandible reddish brown; palpi yellow; legs yellow throughout; A1-A5 yellow, A6 dark brown, remainder of antenna black; fore wing hyaline.

Head. Transverse in dorsal view, 1.44-1.52× as wide as long, slightly wider than mesosoma; lateral ocellus contiguous with inner orbit of compound eye; POL 1.53$1.58 \times$ LOL; occipital carina continuous medially, irregularly crenulate; central keel absent (Plate 49A); medial frons smooth dorsally, punctate rugulose ventrally; ventrolateral frons rugose punctate; frons below median ocellus densely punctate; vertex densely punctate, becoming punctate reticulate posteriorly; gena coarsely punctate reticulate; length of A3 1.28-1.48× length of A2.

Mesosoma. Cervical pronotal area finely punctate; dorsal pronotal area densely coarsely punctate; lateral pronotal area smooth anteriorly, irregularly punctate posteriorly; netrion rugulose; notaulus narrow, foveolate; middle lobe of mesoscutum densely punctate, becoming punctate reticulate anteriorly, interspaces along notaulus in part with microsculpture; lateral lobes of mesoscutum irregularly punctate; mesoscutellum densely punctate throughout; metascutellum transverse (Plate 49B), posterior margin straight or slightly convex medially, longitudinally carinate; propodeum continuous medially (Plate 49B), not divided into two separated lobes, posterior margin narrowly notched medially, each side with several irregular longitudinal carinae medially, otherwise with rugose sculpture covered by dense, recumbent, white setae; upper mesepisternum with a row of robust longitudinal carinae below subalar pit; lower mesepisternum densely punctate to punctate rugulose; mesopleural depression smooth (Plate 49C); metapleuron longitudinally punctate rugulose.

Legs. Slender; hind femur somewhat swollen, 3.57-3.85x as long as its maximum width; hind tibia without spines over outer surface; hind basitarsus $8.18-9.09 \times$ as long as its maximum width.

Wings. Apex of fore wing extending as far as mid-length to anterior $2 / 3$ of T4; R $1.68-1.96 \times$ as long as $r-r s, ~ R 1 ~ 1.64-1.94 \times$ length of $\mathrm{R}$. 


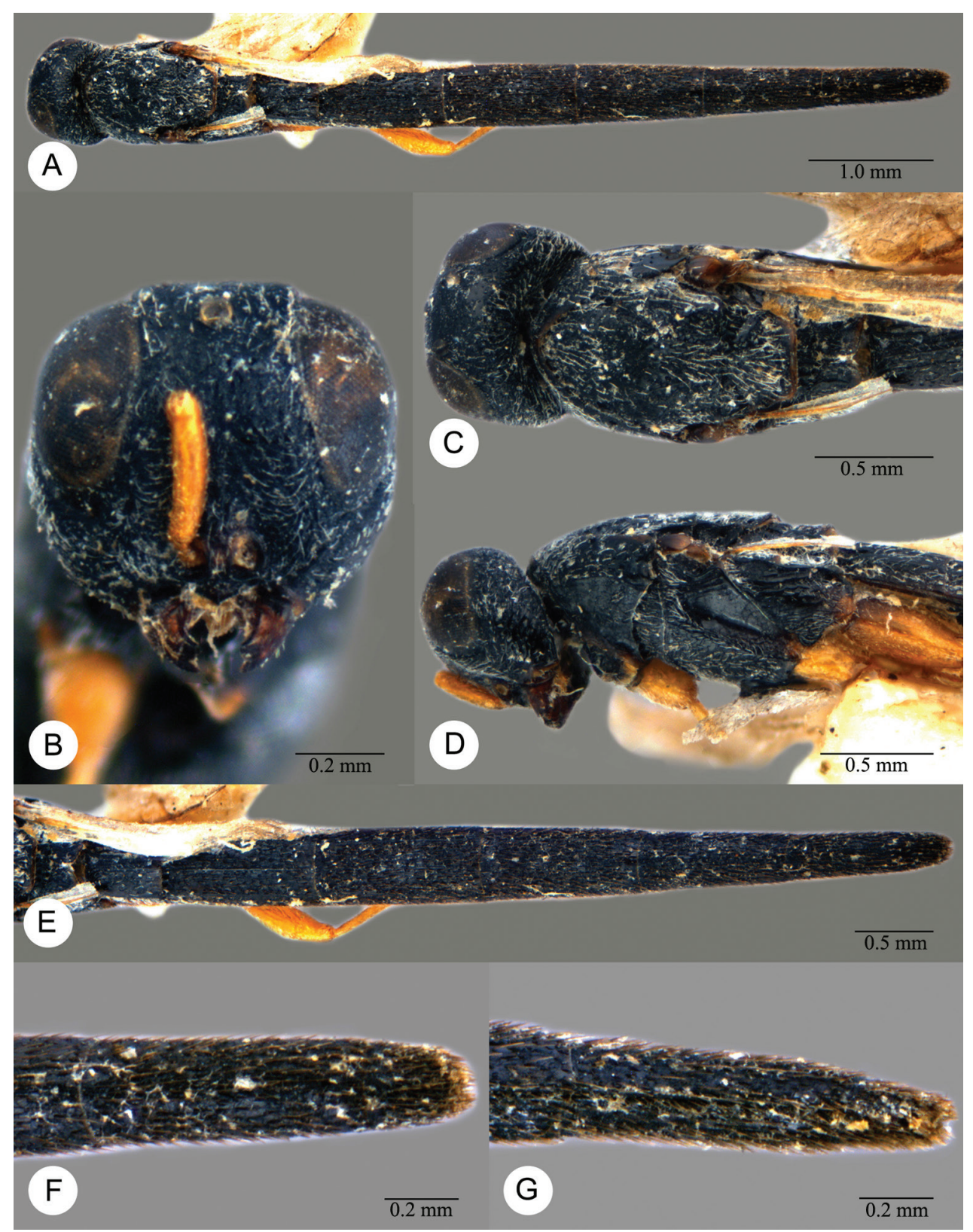

Plate 47. Macroteleia peliades Kozlov \& Lê, holotype, male. A Dorsal habitus B Head, anterior view C Head and mesosoma, dorsal view D Head and mesosoma, lateral view E Metasoma, dorsal view F Apex of metasoma, dorsal view $\mathbf{G}$ Apex of metasoma, lateral view.

Metasoma. Posterior margin of transverse sulcus on T2 straight (Plate 49D); sublateral tergal carinae well developed on T1-T3; T1 longitudinally striate medially, with scattered punctures in interstices anteriorly, punctate rugulose laterally; T2, 


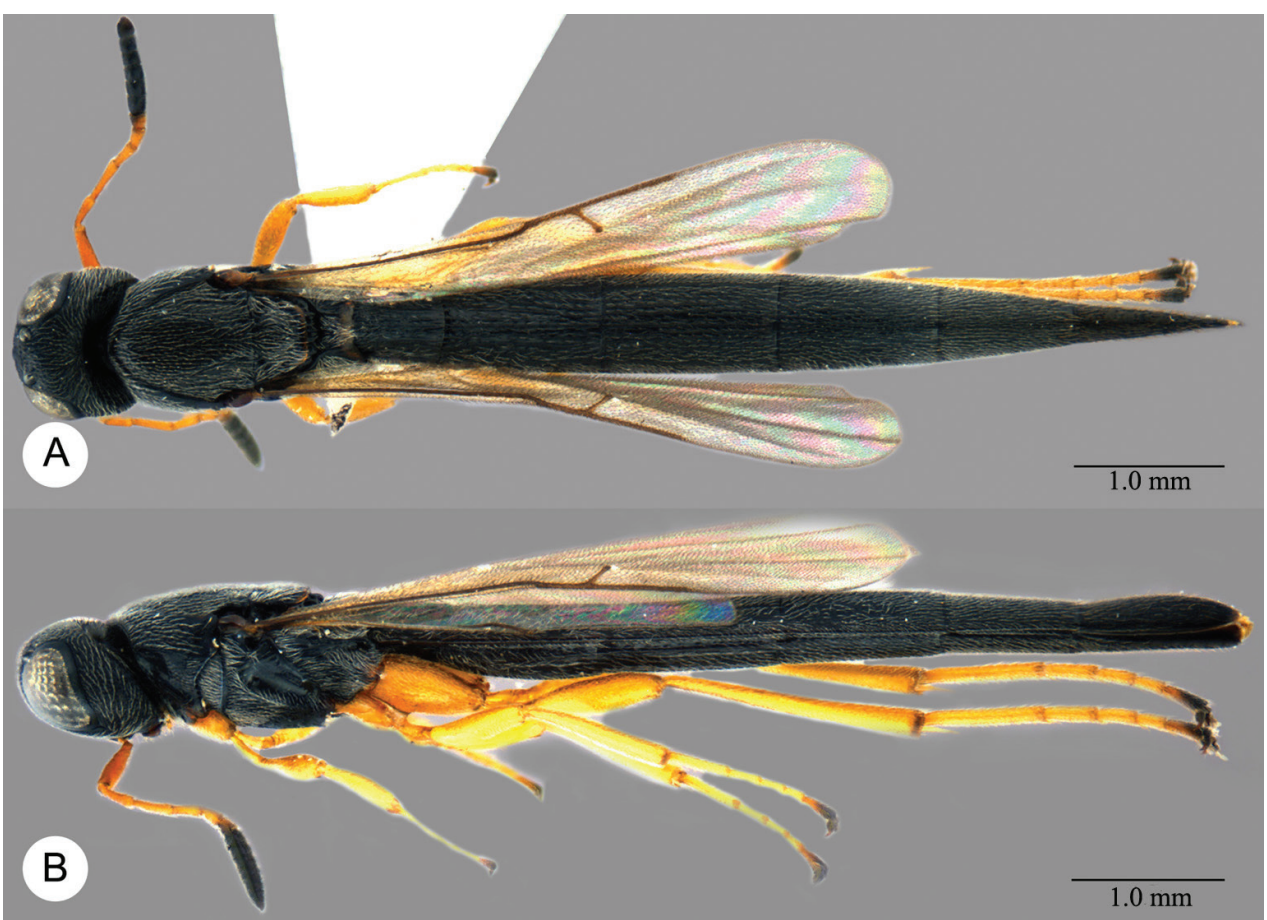

Plate 48. Macroteleia peliades Kozlov \& Lê, female from Guangxi, Longshan Nature Reserve. A Dorsal habitus B Lateral habitus.

T3 longitudinally striate medially, with delicate punctures in interstices, punctate rugulose laterally; T4, T5 longitudinally striate, with numerous delicate punctures in interstices throughout; T6 punctate rugulose dorsally, densely longitudinally striate laterally, with scattered small punctures in interstices ; length of T3 1.03-1.23x length of T6; T5 much longer than wide; S2-S6 sparsely longitudinally striate, with numerous punctures in interstices; prominent longitudinal median carina strongly developed on S2-S5.

Male. Differing from female as follows: body length 7.00-8.00 mm ( $\mathrm{n}=4)$; antenna yellow to brown, becoming darker apically; hind coxa yellow or dark brown to black; T1 sparsely longitudinally striate, with scattered punctures in interstices; T2-T7 densely longitudinally striate, with numerous punctures in interstices; T6 distinctely longer than wide; length of T6 1.30-1.96× length of T7; T7 transverse, apex truncate (Plates 47F, 50B); length of T7 equal to length of S7; S2-S5 sparsely longitudinally striate, with numerous punctures in interstices; S6, S7 longitudinally punctate rugulose.

Diagnosis. This species can be easily separated from the other Chinese Macroteleia by its large size.

Distribution. China (Zhejiang, Guangdong, Guangxi); Vietnam. Link to distribution map [http://hol.osu.edu/map-large.html?id=179764]. 


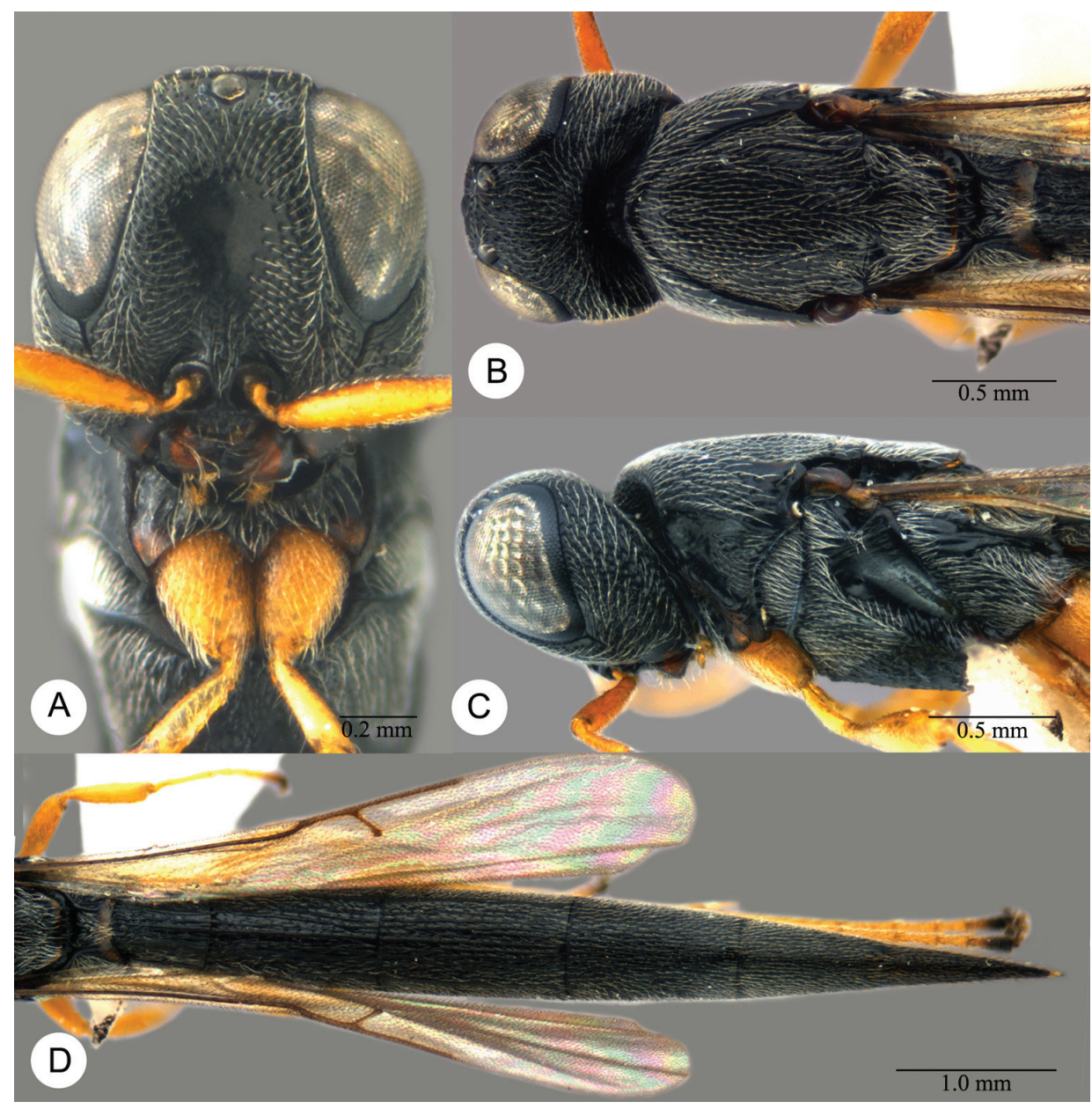

Plate 49. Macroteleia peliades Kozlov \& Lê, female from Guangxi, Longshan Nature Reserve. A Head, anterior view B Head and mesosoma, dorsal view C Head and mesosoma, lateral view D Metasoma, dorsal view.

Material examined. Holotype, ô, VIETNAM: "Van Mai, HSB [=Hoa Binh], 30.V.1982", "Holotypus ô, Macroteleia peliades Kozlov et Lê, 84" (deposited in IEBR).

Other material. CHINA: 1 \%, Zhejiang, Mt. Qingliangfeng, $30^{\circ} 04^{\prime} \mathrm{N}, 118^{\circ} 52^{\prime} \mathrm{E}$, 9.VIII.2005, Min Shi, SCAU 000024 (SCAU); 1 + , Guangdong, Chebaling National Nature Reserve, $24^{\circ} 43^{\prime} \mathrm{N}, 114^{\circ} 14^{\prime} \mathrm{E}, 25 . \mathrm{V} .2002$, Jingxian Liu, SCAU 000025 (SCAU); $1 q+1 \delta^{\jmath}$, Guangdong, Mt. Nankun, $23^{\circ} 37.941^{\prime} \mathrm{N}, 113^{\circ} 50.182^{\prime} \mathrm{E}, 12 . \mathrm{V} .2004$, Zaifu $\mathrm{Xu}, \mathrm{SCAU} 000026,000027$ (SCAU); 1 $+1 \hat{\delta}$, Guangdong, Nanling National Nature

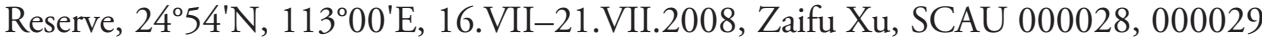
(SCAU); $19+1 \delta^{\lambda}$, Guangxi, Longshan Nature Reserve, 23⒉727' N, 108 $31.918^{\prime} \mathrm{E}$, 1.VII-2.VII.2011, yellow pan trap, Zaifu Xu et al., SCAU 000030, 000031 (SCAU).

Note. Female of this species is described for the first time. 


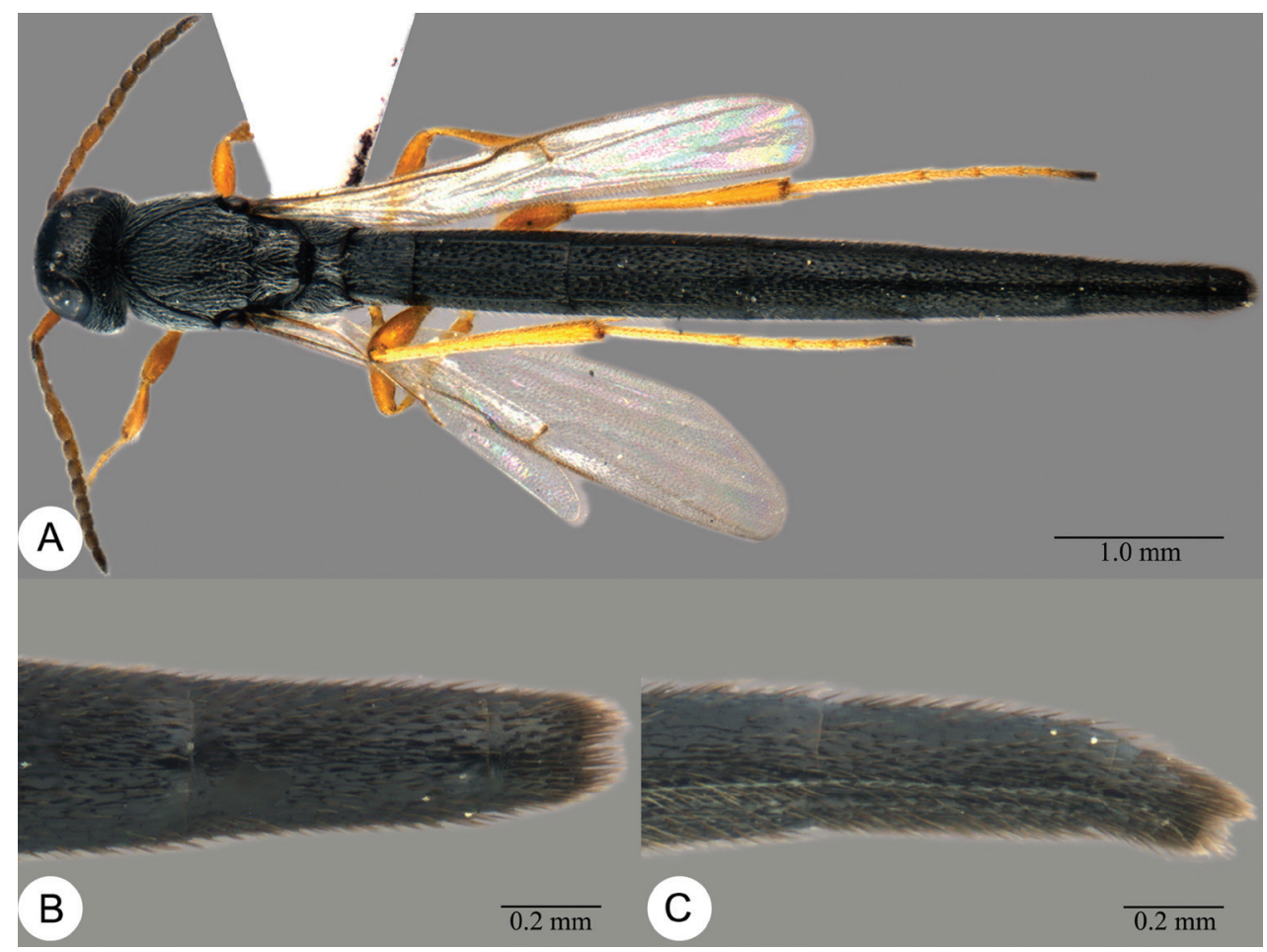

Plate 50. Macroteleia peliades Kozlov \& Lê, male from Guangxi, Longshan Nature Reserve. A Dorsal habitus B Apex of metasoma, dorsal view C Apex of metasoma, lateral view.

\section{Macroteleia rufa Szelényi}

http://species-id.net/wiki/Macroteleia_rufa

Plates $51-52$

Macroteleia rufa Szelényi 1938: 91, 92 (original description); Kozlov and Kononova, 1987: 94, 95 (keyed); Kozlov and Kononova, 1990: 189, 190, 197 (description, keyed); Petrov, 1994: 96 (comparison with Macroteleia angelovi Petrov); Kononova, 1995: 70, 72 (keyed); Kononova and Petrov, 2003: 605 (keyed); Kononova and Kozlov, 2008: 231, 235 (description, keyed, synonym).

Macroteleia eremicola Priesner 1951: 137 (original description); Masner and Muesebeck, 1968: 39 (type information); Kononova and Kozlov, 2008: 235 (junior synonym of Macroteleia rufa Szelényi).

Description. Female. Body length 4.55-6.62 mm (n=10).

Color. Head and mesosoma yellowish brown; metasoma with $\mathrm{T} 1$ and $\mathrm{T} 6$ variably dark brown to black, otherwise yellow; mandible dark brown; palpi yellow; legs yellow throughout; A1 yellow, A2-A5 dark brown, remainder of antenna black; fore wing hyaline. 


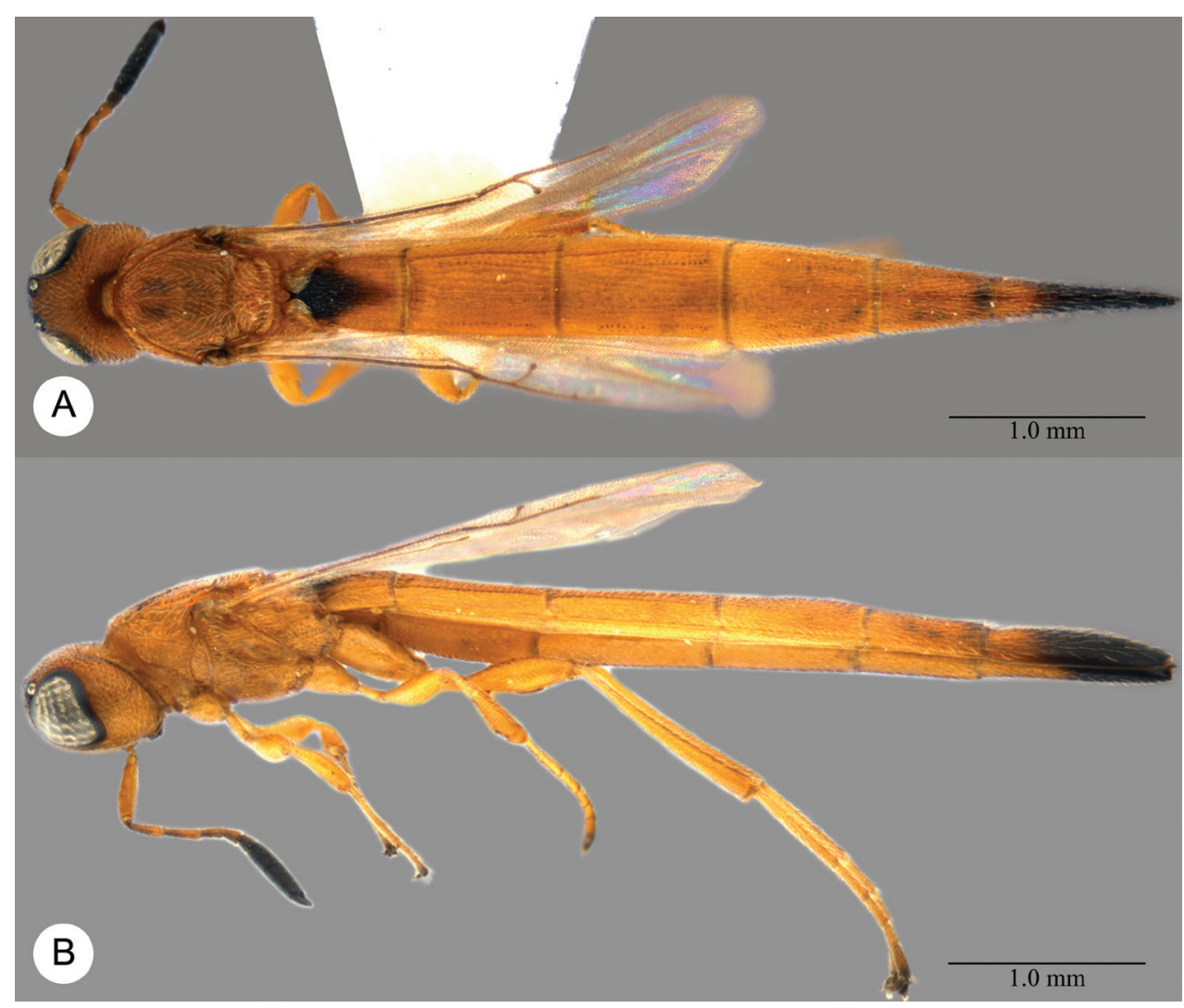

Plate 5 I. Macroteleia rufa Szelényi, female from Guangdong, Guangzhou, Tianlu Lake. A Dorsal habitus B Lateral habitus.

Head. Transverse in dorsal view, $1.30-1.44 \times$ as wide as long, slightly wider than mesosoma; lateral ocellus contiguous with inner orbit of compound eye; POL 1.361.46x LOL; occipital carina continuous medially, irregularly punctate; central keel weakly developed above interantennal process (Plate 52A); medial frons punctate rugulose ventrally, irregularly smooth dorsally; ventrolateral frons punctate rugose; frons below median ocellus densely punctate; vertex densely punctate, interspaces in part with microsculpture; gena punctate rugose; length of A3 1.09-1.19× length of A2.

Mesosoma. Cervical pronotal area densely punctate; dorsal pronotal area areolate rugose; lateral pronotal area longitudinally striate dorsally, punctate rugulose ventrally; netrion punctate rugulose; notaulus narrow, irregularly foveolate; middle lobe of mesoscutum densely punctate, becoming denser anteriorly and at posterior end; lateral lobe of mesoscutum densely punctate, interspaces in part with microsculpture; mesoscutellum densely punctate throughout; metascutellum triangular (Plate 52B), posterior margin strongly produced medially, extending into space between propodeal lobes; propodeum narrowly divided into two subtriangular lobes (Plate 52B), each 


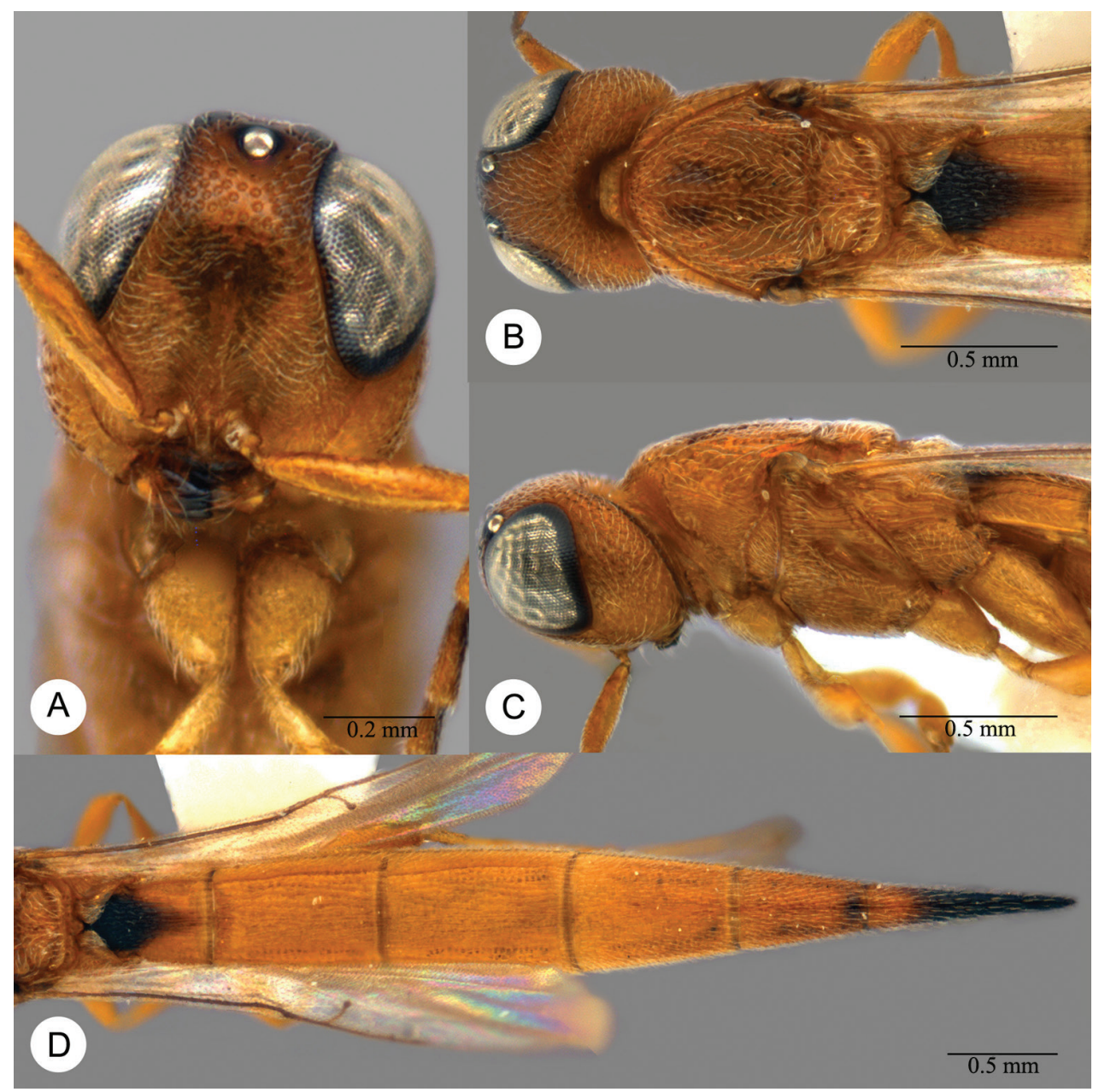

Plate 52. Macroteleia rufa Szelényi, female from Guangdong, Guangzhou, Tianlu Lake. A Head, anterior view B Head and mesosoma, dorsal view C Head and mesosoma, lateral view D Metasoma, dorsal view.

side with several irregular longitudinal carinae medially, otherwise punctate rugulose, covered by dense, recumbent, white setae; upper mesepisternum with a row of strong longitudinal carinae below subalar pit; lower mesepisternum longitudinally punctate rugulose; mesopleural depression smooth (Plate 52C); metapleuron longitudinally striate dorsoventrally, punctate rugulose medially.

Legs. Slender; hind femur weakly swollen, $4.10-4.37 \times$ as long as its maximum width; hind tibia without spines over outer surface; hind basitarsus $10.14-11.33 \times$ as long as its maximum width.

Wings. Apex of fore wing extending from as far as anterior third to mid-length of T4; R 1.38-2.08× as long as r-rs, R1 1.67-2.08× length of R. 
Metasoma. Posterior margin of transverse sulcus on T2 strongly convex (Plate 52D); sublateral tergal carinae developed on T1-T3; T1 densely longitudinally striate medially, with scattered punctures in interstices anteriorly, rugulose laterally; T2-T3 densely longitudinally striate medially, with delicate punctures in interstices, punctate rugulose laterally; T4-T5 densely finely longitudinally striate throughout, with delicate punctures in interstices; T6 finely punctate dorsally, densely longitudinally striate laterally, with scattered small punctures in interstices; length of T3 $0.82-0.94 \times$ length of T6; T5 distinctly longer than wide; S2-S4 longitudinally striate, with finely punctate rugulose interstices; S5-S6 longitudinally striate, with finely punctate interstices; prominent longitudinal median carinae present on S2-S5.

Male. No specimens were available for this study.

Distribution. China (Guangdong, Hainan); Thailand. This species is also recorded in Egypt, Ukraine, Russia, Georgia and Tajikistan. Link to distribution map [http://hol.osu.edu/map-large.html?id=4863].

Material examined. The holotye is deposited in Hungarian Nature History Museum, Budapest.

Other material. CHINA: 1 , , Guangdong, Zijin County, Linjiang Town, 233' $\mathrm{N}$, $114^{\circ} 41^{\prime} \mathrm{E}, 1 . \mathrm{VIII} .2003$, Jingxian Liu, SCAU 000044 (SCAU); 2 ㅇ, Guangdong, Xinfeng, Mt. Yunji, $24^{\circ} 04^{\prime} \mathrm{N}, 114^{\circ} 10^{\prime} \mathrm{E}, 19 . \mathrm{VII} .2003$, Yanxia Song, SCAU 000045,

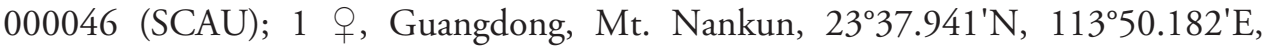
2.VII.2005, Zaifu Xu, SCAU 000047 (SCAU); 4 + , Guangdong, Guangzhou, Tianlu Lake, $23^{\circ} 13^{\prime} \mathrm{N}, 113^{\circ} 25^{\prime} \mathrm{E}, 6 . X .2002$, Zaifu Xu, SCAU 000048-000051 (SCAU); 1 , Hainan, Mt. Yinggeling, $18^{\circ} 49^{\prime} \mathrm{N}, 109^{\circ} 11^{\prime} \mathrm{E}, 17-20 . V I I .2010$, Huayan Chen, SCAU 000052 (SCAU). THAILAND: 1 \%, Chiang Mai, Maerim, 10-12.III.2003, MT, R. A. Beaver, No. 26778 (RABC).

Comments. The identification of this species is based on pictures of holotype of $M$. rufa (deposited in Hungarian Nature History Museum, Budapest) kindly provided by Dr. Ovidiu A. Popovici (University 'Al. I. Cuza' Iaşi) and his personal communication.

\section{Macroteleia salebrosa sp. $\mathbf{n}$.}

urn:Isid:zoobank.org:act:2016B64A-968B-4C39-A51F-DB4CDD750F75

http://species-id.net/wiki/Macroteleia_salebrosa

Plates 53-55

Description. Female. Body length 4.76-5.90 mm ( $\mathrm{n}=6)$.

Color. Body black; mandible reddish brown to black; palpi yellow; hind coxa dark brown to nearly black, remainder of legs yellow to light brown; A1-A6 yellow, remainder of antenna dark brown to black; fore wing hyaline.

Head. Transverse in dorsal view, $1.40-1.54 \times$ as wide as long, slightly wider than mesosoma; lateral ocellus contiguous with inner orbit of compound eye; POL 1.73$1.83 \times$ LOL; occipital carina interrupted medially; central keel well developed, extend- 


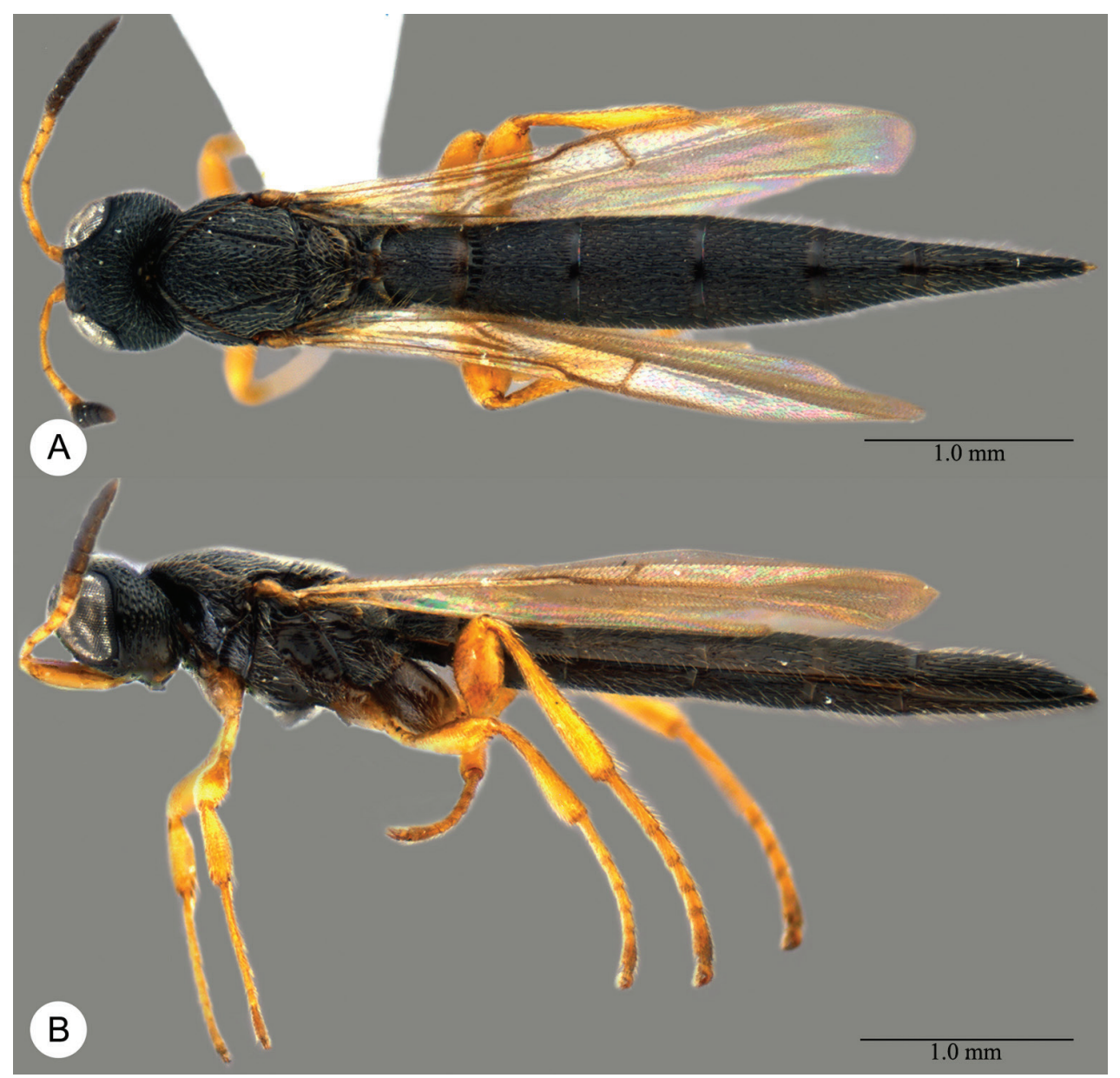

Plate 53. Macroteleia salebrosa sp. n., holotype, female. A Dorsal habitus B Lateral habitus.

ing onto interantennal process (Plate 54A); medial frons smooth dorsally, obliquely strigose ventrally; ventrolateral frons punctate rugose; frons below median ocellus and vertex punctate reticulate; gena dorsoventrally strigose below malar sulcus, otherwise punctate rugose; length of A3 1.07-1.15x length of A2.

Mesosoma. Cervical pronotal area densely punctate; dorsal pronotal area areolate; lateral pronotal area smooth dorsally, rugulose ventrally; netrion punctate rugulose; notaulus narrow, foveolate; middle lobe of mesoscutum densely punctate, becoming reticulate anteriorly; lateral lobes of mesoscutum moderately punctate throughout; mesoscutellum densely punctate throughout; metascutellum rectangular (Plate 54B), posterior margin slightly convex, extending to gap between propodeal lobes, irregularly areolate rugose; propodeum medially divided into two widely separated subtriangular lobes (Plate 54B), each side with rugose sculpture covered by dense, recumbent, white setae; upper mesepisternum with a row of fine longitudinal carinae below subalar pit; 


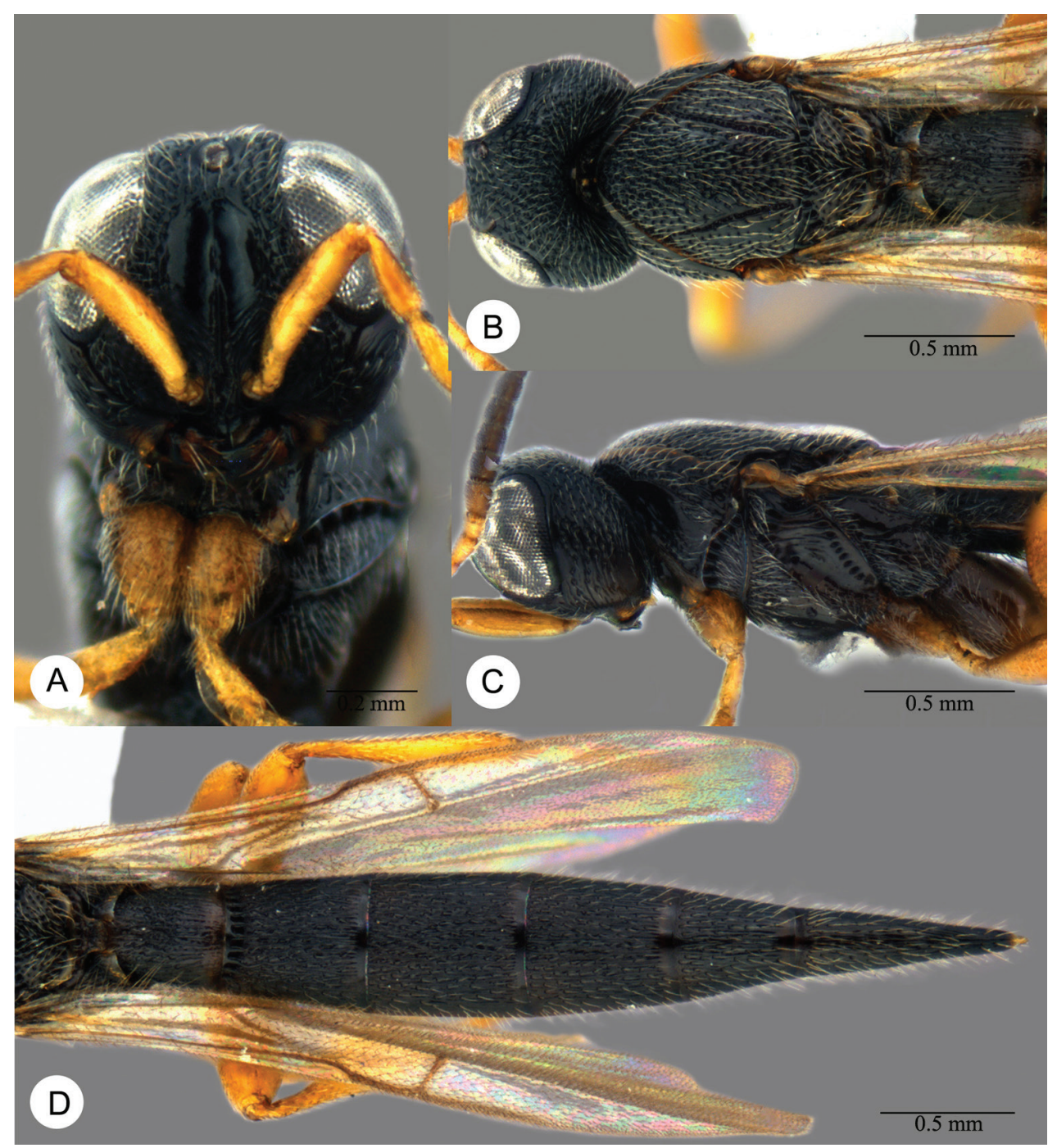

Plate 54. Macroteleia salebrosa sp. n., holotype, female. A Head, anterior view B Head and mesosoma, dorsal view C Head and mesosoma, lateral view D Metasoma, dorsal view.

lower mesepisternum variably smooth to punctate rugulose; mesopleural depression smooth (Plate 54C); metapleuron smooth dorsally, punctate rugulose ventrally.

Legs. Robust; hind femur strongly swollen, $2.47-2.94 \times$ as long as its maximum width; hind tibia without spines over outer surface; hind basitarsus $4.50-5.00 \times$ as long as its maximum width.

Wings. Apex of fore wing extending beyond posterior margin of T5; R 1.50-1.89× as long as r-rs, R1 1.47-1.77× length of R.

Metasoma. Posterior margin of transverse sulcus on T2 slightly convex (Plate 54D); sublateral tergal carinae obscured by longitudinal surface striae; T1 punctate 


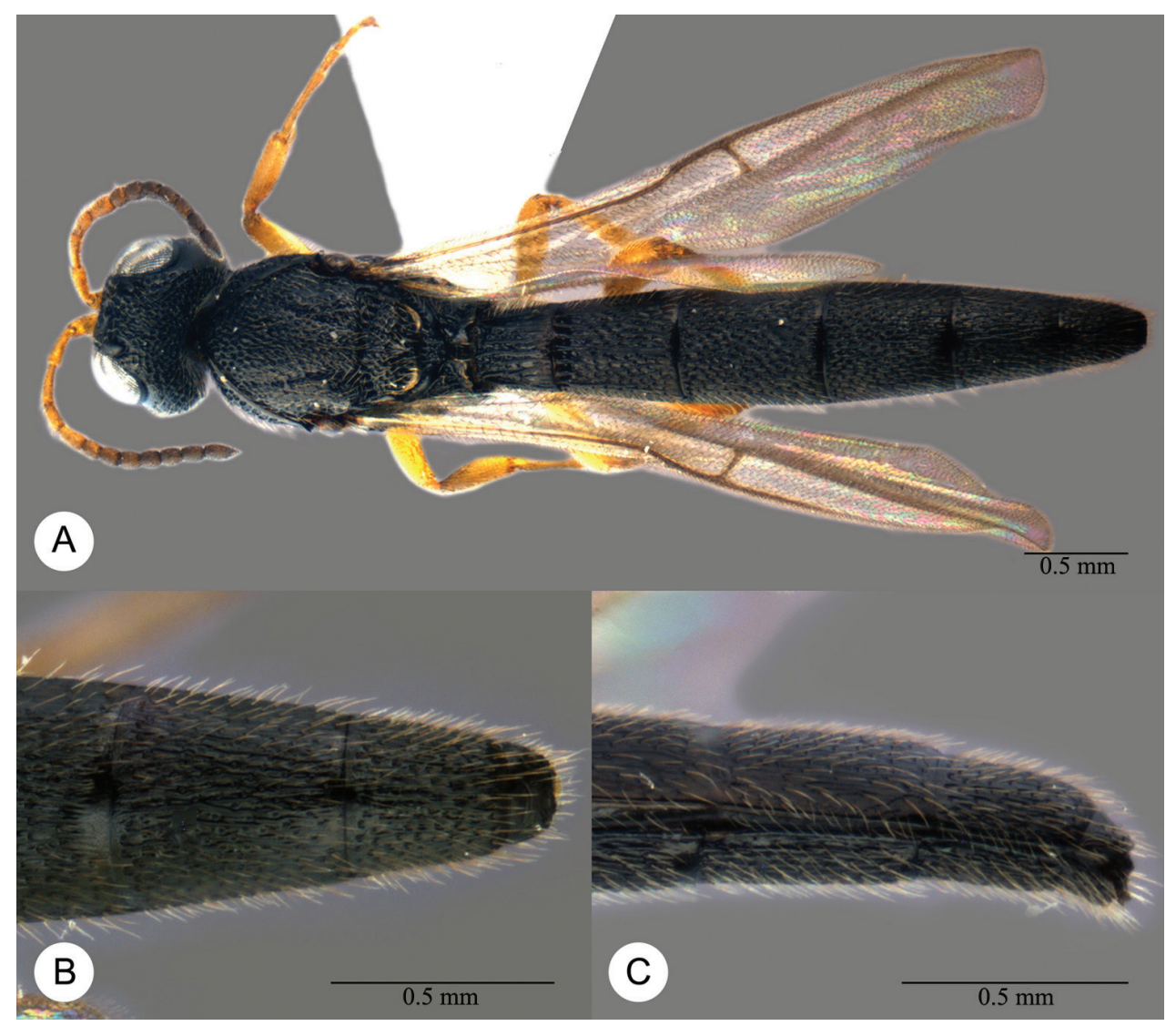

Plate 55. Macroteleia salebrosa sp. n., paratype, male. A Dorsal habitus B Apex of metasoma, dorsal view C Apex of metasoma, lateral view.

rugose anteriorly, densely striate posteriorly; T2-T5 densely longitudinally striate with numerous large delicate punctures in interstices; T6 punctate rugulose dorsally, longitudinally striate laterally, with scattered small punctures in interstices; length of T3 0.69-0.78x length of T6; T5 slightly longer than wide; S2-S6 densely longitudinally striate, with numerous punctures in interstices; prominent longitudinal median carina obscured by longitudinal surface striae.

Male. Differing from female as follows: body length $5.00 \mathrm{~mm}(\mathrm{n}=2)$; A1-A5 yellow to brown, A6-A12 dark brown, becoming darker apically; propodeum continuous medially (Plate 55A), not divided into two separated lobes, posterior margin excavate medially, each side with several irregular longitudinal carinae medially, otherwise rugose; sublateral tergal carinae developed on T1-T2; T1 sparsely longitudinally striate, with scattered punctures in interstices anteriorly; T2-T6 longitudinally punctate rugulose; T7 transversely striate posteriorly; T6 slightly longer than wide; length of T6 2.56-2.86x length of T7; T7 transverse, apex truncate (Plate 55B); length of T7 
0.83-0.84x length of S7; S2-S7 densely longitudinally striate, with numerous punctures in interstices; prominent longitudinal median carina present on S2-S4.

Etymology. The name salebrosa refers to the coarse body sculpture of this species and is used as an adjective.

Distribution. China (Zhejiang, Sichuan). Link to distribution map [http://hol. osu.edu/map-large.html?id=320506].

Material examined. Holotype, + : CHINA: Zhejiang, Mt. Qingliangfeng, 9.VIII.2005, 3004'N, 118 52'E, Hongying Zhang, SCAU 000016 (deposited in SCAU). Paratypes: 1 , Zhejiang, Mt. Tianmu, Xianrending, 30 20.56'N, $119^{\circ} 26.03$ 'E, 25-29.VII.2011, sweeping, Chengyuan Jin, SCAU 000017 (SCAU); 2 §., Zhejiang, Mt. Tianmu, Xianrending, 30²0.56'N, $119^{\circ} 26.03^{\prime} \mathrm{E}, 25-29 . V I I .2011$, sweeping, Huayan Chen, SCAU 000018, 000019 (SCAU); 1 q, Zhejiang, Mt. Baishanzu, $27^{\circ} 45^{\prime} \mathrm{N}, 119^{\circ} 12^{\prime} \mathrm{E}, 1856 \mathrm{~m}, 15 . \mathrm{VIII} .2003$, Jingxian Liu, SCAU 000020 (SCAU); 3 ㅇ, Sichuan, Mt. Emei, $29^{\circ} 34^{\prime} \mathrm{N}, 103^{\circ} 26^{\prime} \mathrm{E}, 2$.VIII.2006, Hongying Zhang, SCAU 000021-000023 (SCAU).

\section{Macroteleia semicircula sp. n.}

urn:lsid:zoobank.org:act:5813FBA4-90E4-4A67-8ECF-A87E6EB7AF6E

http://species-id.net/wiki/Macroteleia_semicircula

Plates 56-58

Description. Female. Body length 5.83-6.36 mm ( $\mathrm{n}=4)$.

Color. Head yellow with upper frons and vertex dark brown to black; mesosoma variably yellow to dark brown; metasoma black; mandible dark brown; palpi yellow; legs yellow throughout; A1-A6 yellow, A7-A12 black; fore wing subhyaline.

Head. Transverse in dorsal view, $1.42-1.46 \times$ as wide as long, slightly wider than mesosoma; lateral ocellus contiguous with inner orbit of compound eye; POL 1.541.55x LOL; occipital carina continuous medially, coarsely and densely punctate; central keel well developed, extending onto interantennal process, slightly bifurcating dorsally (Plate 57A); medial frons smooth; ventrolateral frons punctate rugose; frons below median ocellus and vertex punctate reticulate; gena punctate rugose; length of A3 1.16-1.18x length of A2.

Mesosoma. Cervical pronotal area densely punctate; dorsal pronotal area areolate; lateral pronotal area anteriorly with several carinae subparallel to vertical epomial carina, otherwise smooth with sparse punctures; netrion finely punctate; notaulus deep, distinctly foveolate; mesoscutum densely punctate throughout; mesoscutellum sparsely punctate medially, densely punctate laterally; metascutellum semicircular (Plate 57B), extending to gap between propodeal lobes, irregularly areolate rugose; propodeum divided into two widely separated subtriangular lobes (Plate 57B), each side with rugose sculpture covered by dense, recumbent, white setae; upper mesepisternum with a row of robust longitudinal carinae below subalar pit; lower mesepisternum variably smooth to punctate rugulose; mesopleural depression smooth (Plate 57C); metapleuron smooth dorsally, punctate rugose ventrally. 


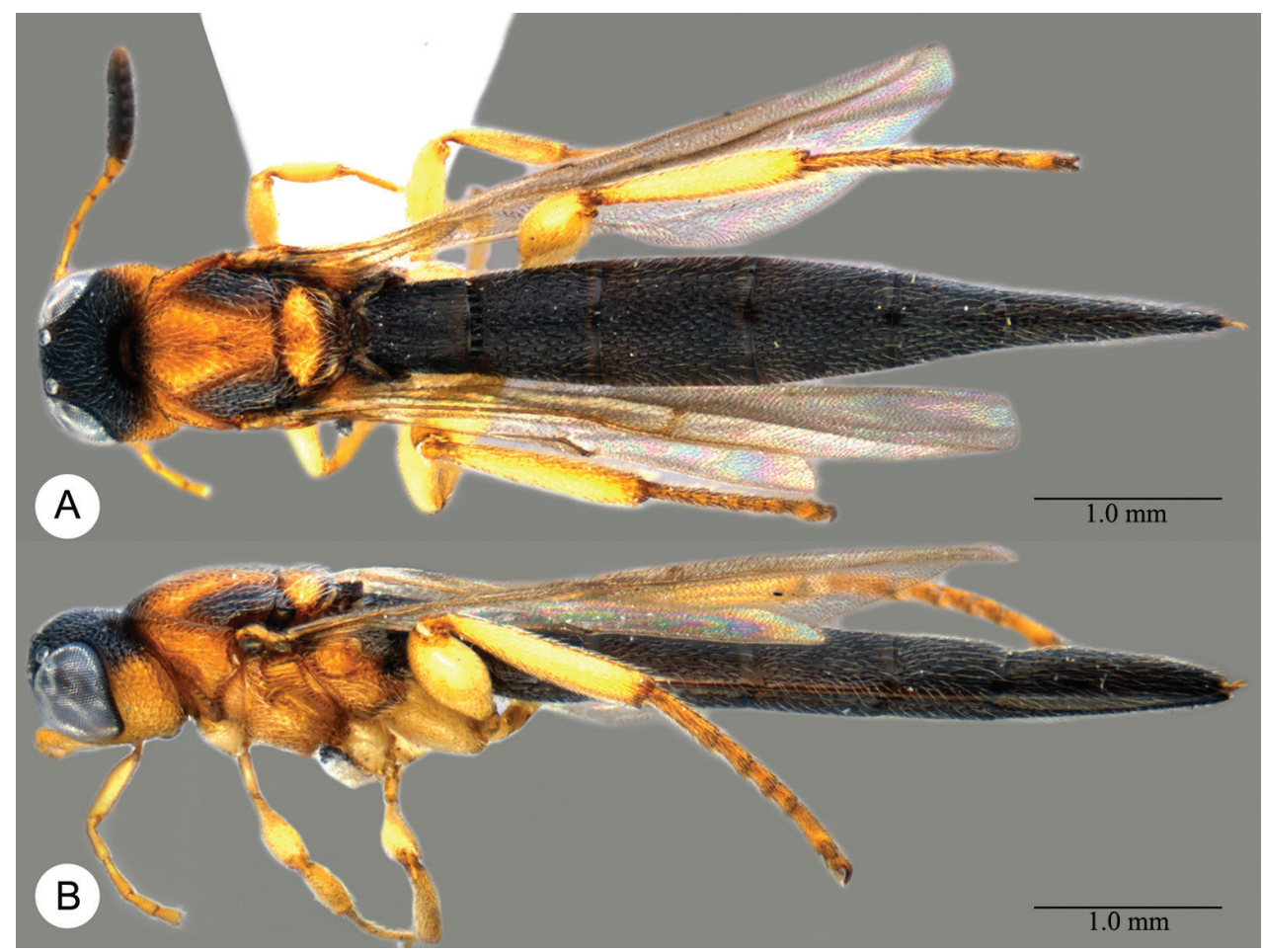

Plate 56. Macroteleia semicircula sp. n., holotype, female. A Dorsal habitus B Lateral habitus.

Legs. Robust; hind femur strongly swollen, $2.31-2.46 \times$ as long as its maximum width; hind tibia without spines over outer surface; hind basitarsus $3.63-4.23 \times$ as long as its maximum width.

Wings. Apex of fore wing extending as far as mid-length of T5; R 1.38-1.60x as long as r-rs, R1 1.81-2.00× length of R.

Metasoma. Posterior margin of transverse sulcus on T2 strongly convex (Plate 57D); sublateral tergal carinae developed on T1-T3; T1 longitudinally punctate rugulose anteriorly, densely striate posteriorly; T2-T4 longitudinally punctate rugulose throughout; T5 -T6 finely punctate rugulose dorsally, densely longitudinally striate laterodorsally, with scattered small punctures in interstices; length of T3 0.63-0.66× length of T6; T5 slightly longer than wide; S2-S6 densely longitudinally striate, with coarse punctures in interstices; prominent longitudinal median carina present on S2-S4.

Male. Differing from female as follows: body length 4.12-5.56 mm ( $\mathrm{n}=8)$; antenna yellow with A6-A11 brown; mesosoma variably brown to nearly black; fore wing slightly infuscate in basal half; propodeum continuous medially (Plate 58A), not divided into two separated lobes, posterior margin excavate medially, each side with several irregular longitudinal carinae medially, otherwise rugose; T1 sparsely longitudinally striate throughout; T7 largely smooth except finely rugulose posterolaterally; T6 wider than long or slightly longer than wide; length of T6 3.80-4.75× length of 


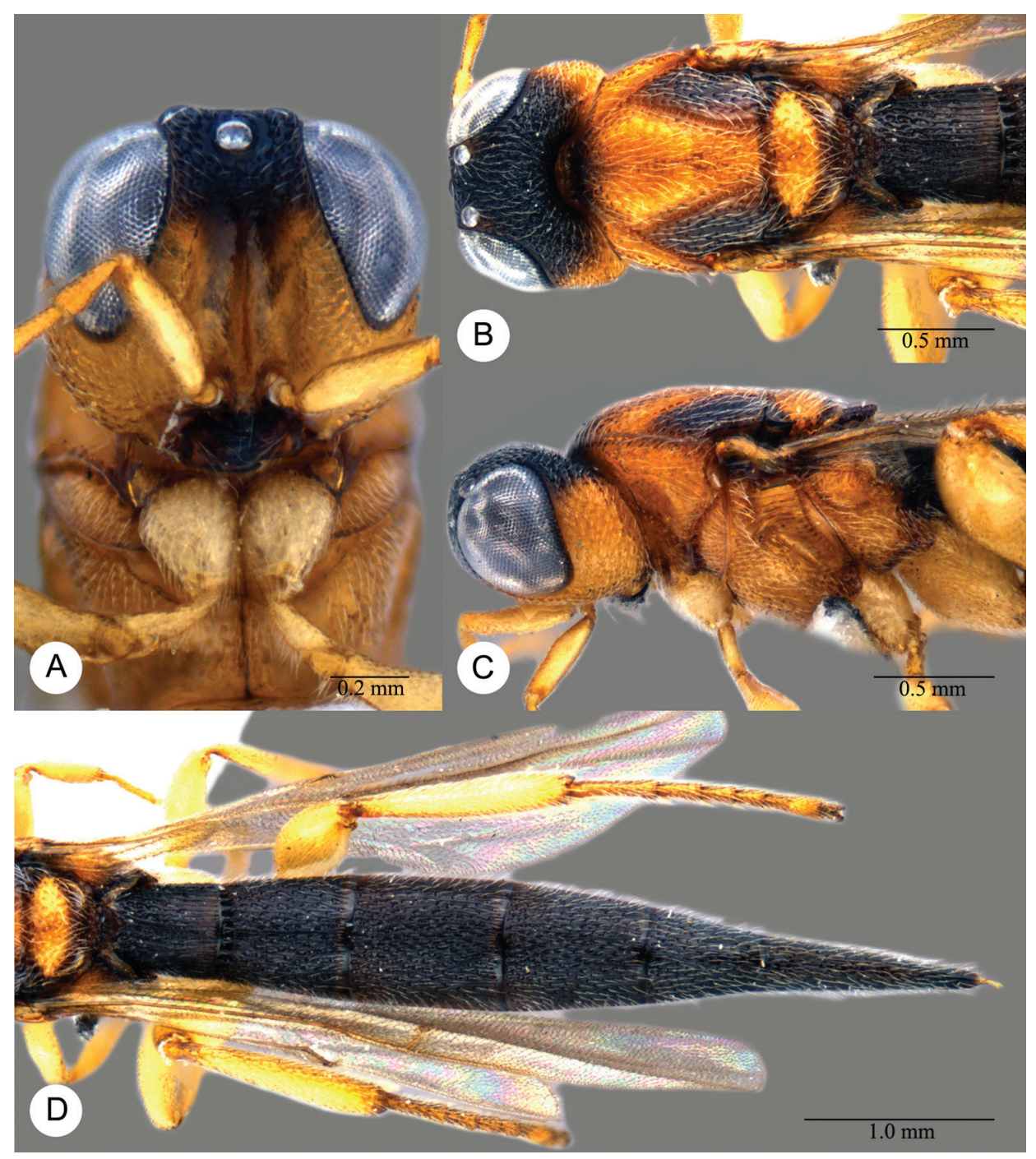

Plate 57. Macroteleia semicircula sp. n., holotype, female. A Head, anterior view B Head and mesosoma, dorsal view C Head and mesosoma, lateral view D Metasoma, dorsal view.

T7; T7 transverse, apex truncate (Plate 58B); length of T7 $0.67-0.85 \times$ length of S7; S7 longitudinally punctate rugulose.

Diagnosis. Macroteleia semicircula shares the well-developed central keel and robust legs with $M$. salebrosa and M. striatipleuron, but can be distinguished by the semicircular metascutellum (rectangular in the latter two species).

Etymology. The name semicircula refers to the semicircular metascutellum of this species and is used as a noun in apposition.

Distribution. China (Guangdong, Hainan). Link to distribution map [http://hol. osu.edu/map-large.html?id=320507]. 


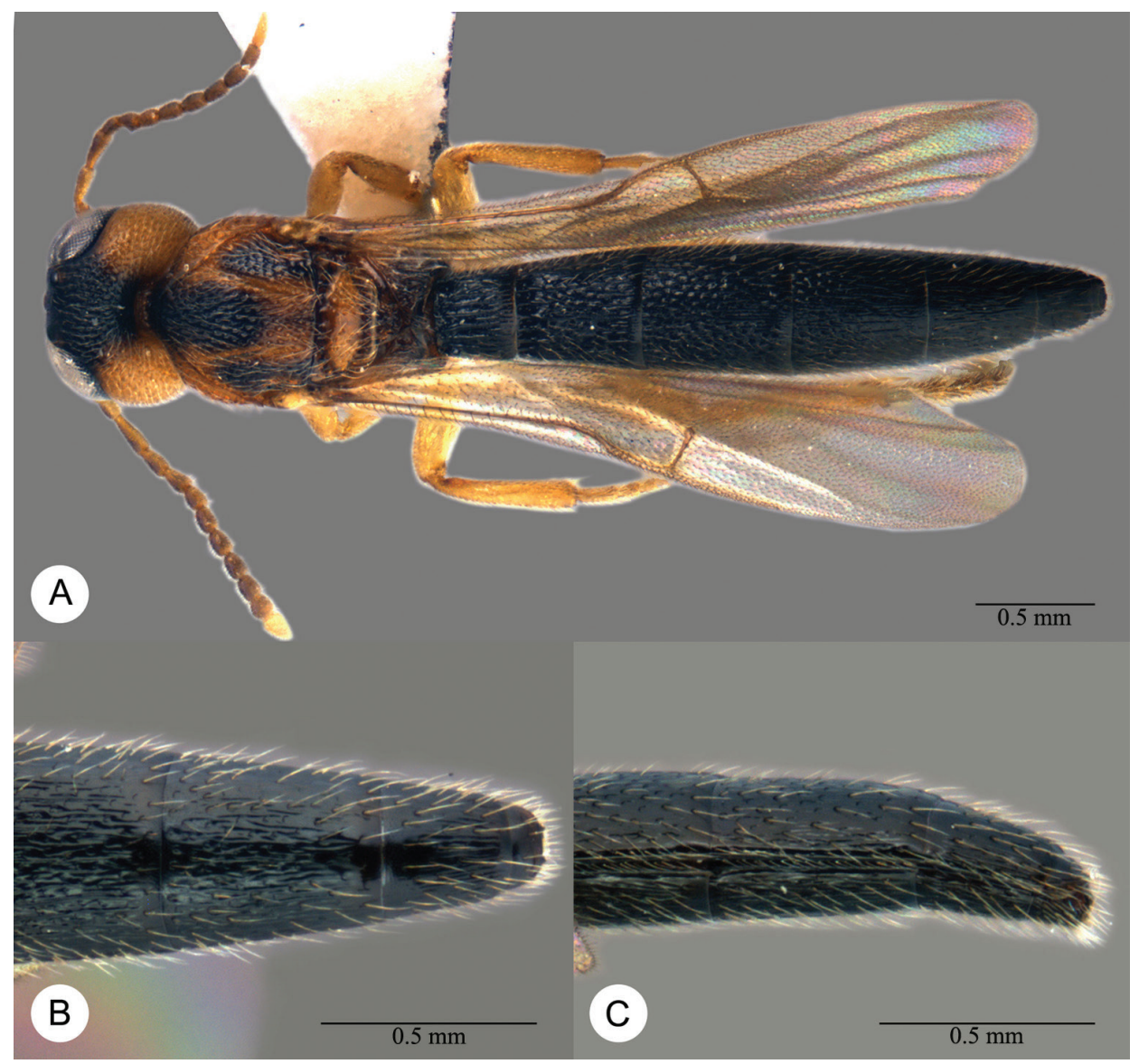

Plate 58. Macroteleia semicircula sp. n., paratype, male. A Dorsal habitus B Apex of metasoma, dorsal view C Apex of metasoma, lateral view.

Material examined. Holotype, + : CHINA: Guangdong, Nanling National Nature Reserve, $24^{\circ} 54^{\prime} \mathrm{N}, 113^{\circ} 00^{\prime} \mathrm{E}, 8-17 . V I I I .2010$, sweeping, Huayan Chen, SCAU 000001 (deposited in SCAU). Paratypes: $2{ }^{\top}$, Guangdong, Nanling National Nature Reserve, $24^{\circ} 54^{\prime} \mathrm{N}, 113^{\circ} 00^{\prime E}$, 9-18.VII.2004, Juanjuan Ma, SCAU 000002, 000003 (SCAU); 1 O , Hainan, Bawangling National Nature Reserve, $19^{\circ} 07^{\prime} \mathrm{N}, 109^{\circ} 03^{\prime} \mathrm{E}, 21 . \mathrm{X} .2007$, Jiemin Yao, SCAU 000004 (SCAU); $1+1+\hat{\sigma}$, Hainan, Bawangling National Nature Reserve, $19^{\circ} 07^{\prime} \mathrm{N}, 109^{\circ} 03^{\prime} \mathrm{E}, 21 . \mathrm{V} .2007$, Jingxian Liu, SCAU 000005, 000006 (SCAU); 1 đo, Hainan, Wuzhishan National Nature Reserve, $18^{\circ} 51^{\prime} \mathrm{N}, 109^{\circ} 39^{\prime} \mathrm{E}, 16$ 18.V.2007, Liqiong Weng, SCAU 000007 (SCAU); 1 ふ̋, Hainan, Wuzhishan National Nature Reserve, 16-18.V.2007, 1851'N, 109³9'E, Jingxian Liu, SCAU 000008 (SCAU); 1 , Hainan, Mt. Yinggeling, 18 $49^{\prime} \mathrm{N}, 109^{\circ} 11^{\prime} \mathrm{E}, 24-25 . X .2007$, Jingxian Liu, SCAU 000009 (SCAU); $1 \delta^{\top}$, Hainan, Mt. Yinggeling, 1849'N, 109¹1'E, 2225.V.2007, Jie Zeng, SCAU 000010 (SCAU); 2 đ̃, Hainan, Mt. Yinggeling, 1849'N, $109^{\circ} 11^{\prime} \mathrm{E}, 28 . X .2007$, Liqiong Weng, SCAU 000011, 000012 (SCAU). 


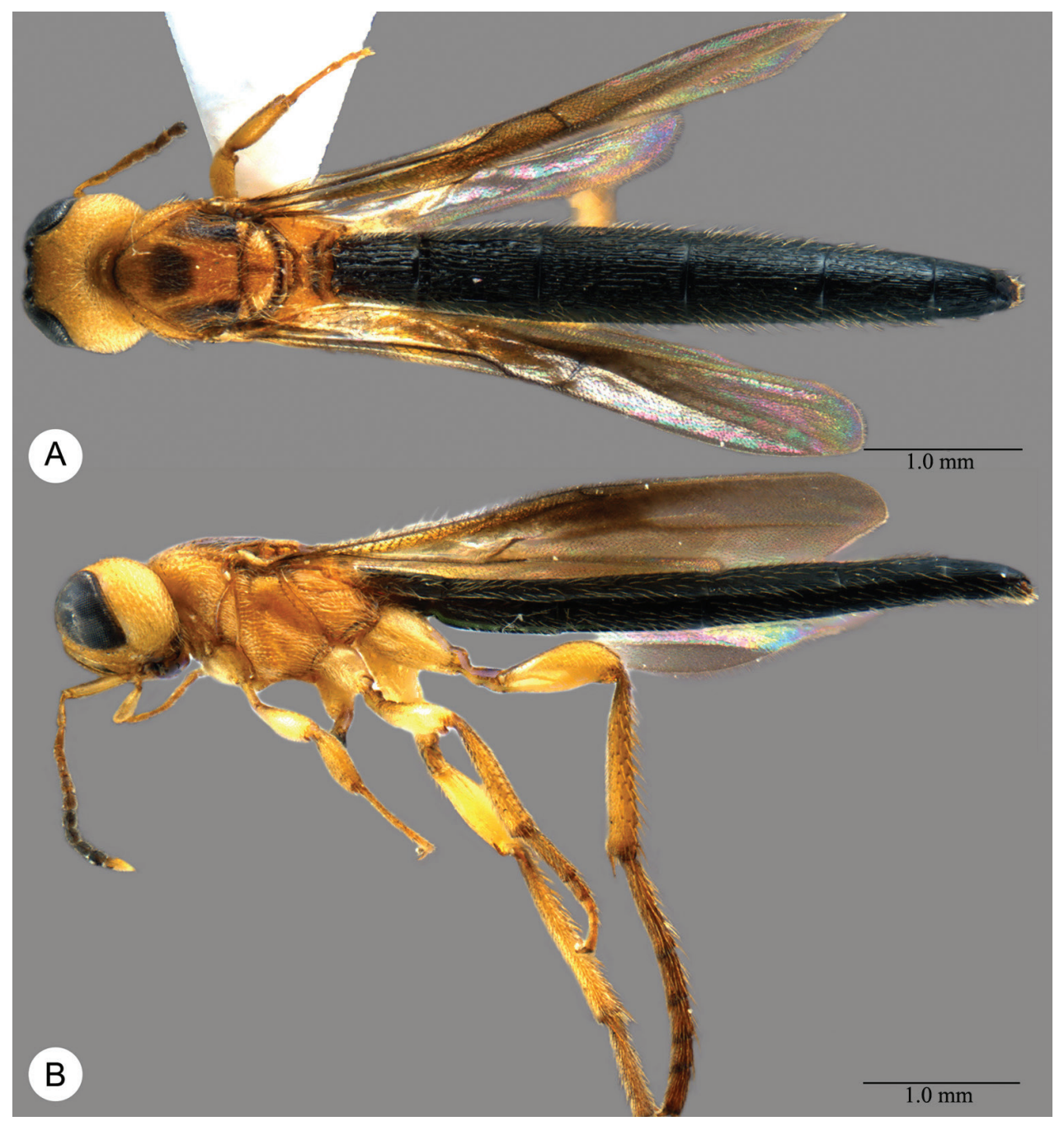

Plate 59. Macroteleia spinitibia sp. n., holotype, male. A Dorsal habitus B Lateral habitus.

\section{Macroteleia spinitibia sp. $\mathrm{n}$.}

urn:lsid:zoobank.org:act:86880BD0-AC42-48C1-A096-D6A1B191C7EE http://species-id.net/wiki/Macroteleia_spinitibia

Plates 59-61

Description. Male. Body length 6.08-6.17 mm ( $\mathrm{n}=2)$.

Color. Head yellow; mesosoma variably yellow to brown; metasoma black; mandible dark brown; palpi yellow; legs yellow with hind tarsi brown; A1 and A12 yellow, A2-A7 brown, A8-A11 dark brown; fore wing hyaline with medial longitudinal infuscate streak extending to near apex of wing (Plate 60D). 


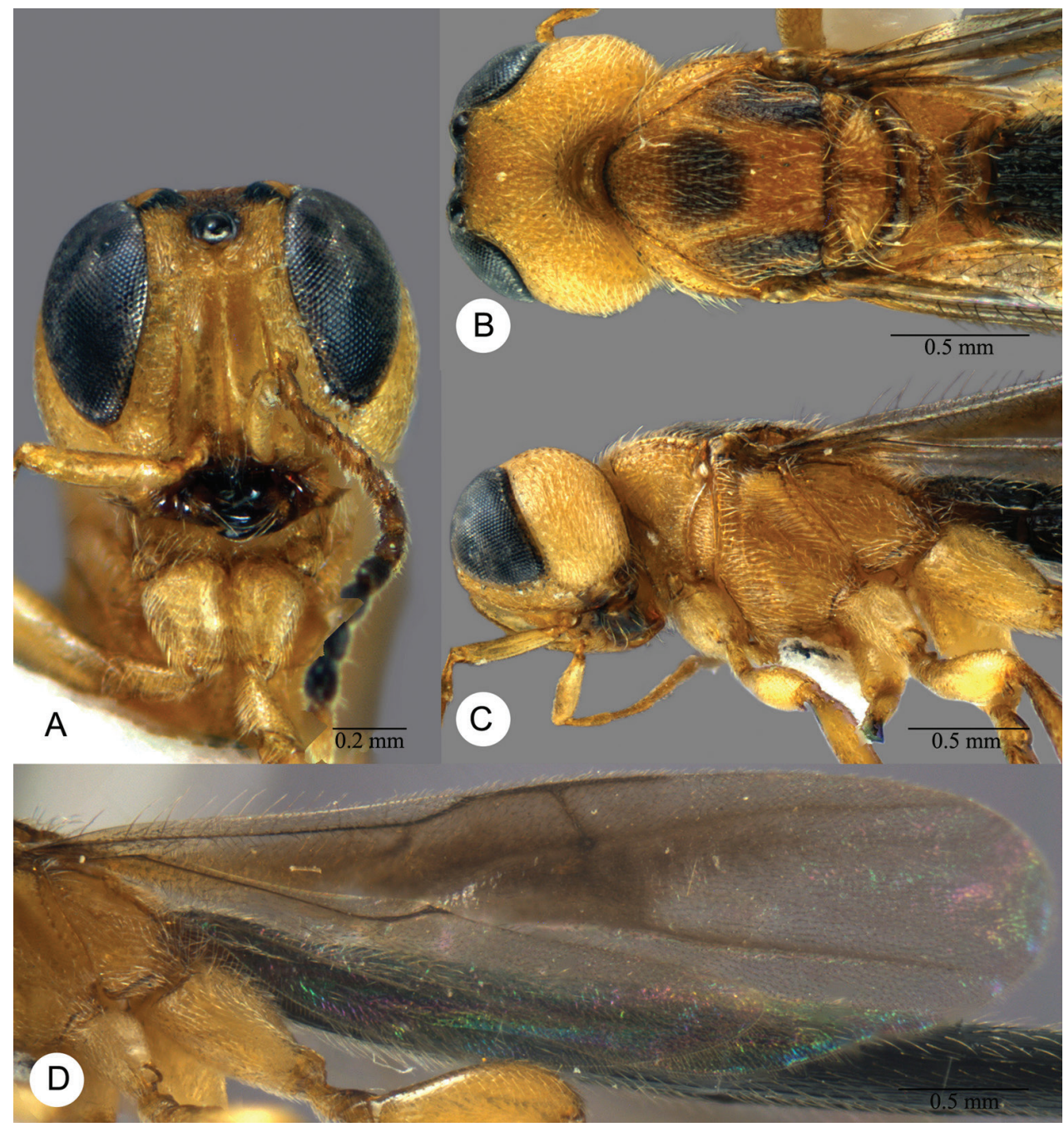

Plate 60. Macroteleia spinitibia sp. n., holotype, male. A Head, anterior view B Head and mesosoma, dorsal view $\mathbf{C}$ Head and mesosoma, lateral view $\mathbf{D}$ Fore and hind wing.

Head. Transverse in dorsal view, $1.38-1.40 \times$ as wide as long, slightly wider than mesosoma; lateral ocellus contiguous with inner orbit of compound eye; POL 1.81$1.88 \times$ LOL; occipital carina continuous medially, densely punctate; central keel well developed (Plate 60A), extending onto interantennal process; medial frons largely smooth, finely strigose dorsally; remainder of frons punctate rugulose; vertex punctate reticulate, narrow interspaces with fine microsculpture; gena punctate rugulose; length of A3 $1.04 \times$ length of A2.

Mesosoma. Cervical pronotal area punctate rugulose; dorsal pronotal area areolate; lateral pronotal area smooth anteriorly, densely punctate posteriorly; netrion rugulose; notaulus deep, distinctly foveolate; middle lobe of mesoscutum punctate reticulate throughout, 


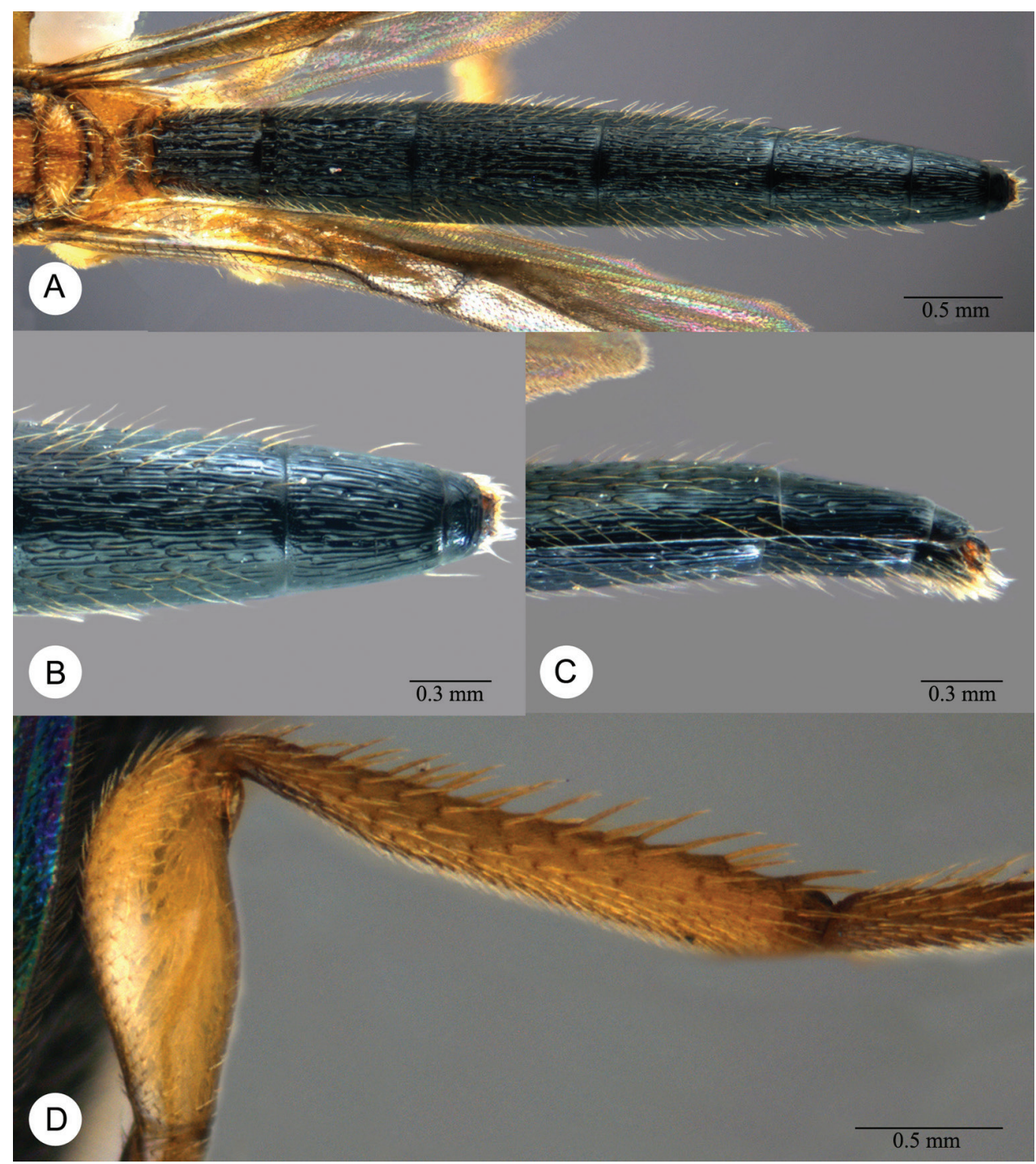

Plate 6I. Macroteleia spinitibia sp. n., holotype, male. A Metasoma, dorsal view B Apex of metasoma, dorsal view $\mathbf{C}$ Apex of metasoma, lateral view D Hind femur and tibia.

narrow interspaces in part with fine microsculpture; lateral lobes of mesoscutum sparsely punctate throughout, interspaces with fine microsculpture; mesoscutellum densely punctate throughout; metascutellum transverse (Plate 60B), posterior margin concave medially, irregularly rugose; propodeum continuous medially, not divided into two separated lobes (Plate 60B), posterior margin convex, each side with several irregular longitudinal carinae medially, otherwise rugose; upper mesepisternum with a row of longitudinal fine striae below subalar pit; lower mesepisternum punctate rugulose; mesopleural depression longitudinally striate (Plate 60C); metapleuron longitudinally striate throughout. 
Legs. Robust; hind femur strongly swollen, $2.86 \times$ as long as its maximum width; hind tibia with numerous semi-erect, yellow spines over outer surface (Plate 61D); hind basitarsus 6.53-6.67x as long as its maximum width.

Wings. Apex of fore wing extending as far as basal third of T5; R 1.50-1.53× as long as r-rs, R1 1.04-1.53× length of R.

Metasoma. Posterior margin of transverse sulcus on T2 straight (Plate 61A); sublateral tergal carinae well developed on T1-T2; T1 sparsely longitudinally striate, with delicate punctures in interstices; T2-T6 densely longitudinally striate, with numerous delicate punctures in interstices; T7 finely rugulose posterolaterally; T6 slightly longer than wide; length of T6 4.00-4.55x length of T7; T7 transverse, apex truncate (Plate 61B); length of T7 0.61-0.71× length of S7; S2-S6 densely longitudinally striate, with numerous punctures in interstices; $S 7$ densely punctate; prominent longitudinal median carina present on S2-S6.

Female. Unknown.

Diagnosis. The combination of infuscate fore wing, short R1 and spinulate hind tibia renders this species rather straightforward to identify.

Etymology. The name spinitibia refers to hind tibia with numerous semi-erect spines over outer surface in this species. The epithet is used as a noun in apposition.

Distribution. China (Hainan). Link to distribution map [http://hol.osu.edu/ map-large.html?id=320508].

Material Examined. Holotype, $\delta^{\lambda}$ : CHINA: Hainan, Bawangling National Nature Reserve, $19^{\circ} 07^{\prime} \mathrm{N}, 109^{\circ} 03^{\prime} \mathrm{E}, 7-11 . \mathrm{VII} .2006$, Jingxian Liu, SCAU 000014 (deposited in SCAU). Paratype: $1 \mathrm{O}^{\top}$, Hainan, Mt. Yinggeling, 18²49'N, 109¹1'E, 16.XI.2008, Manman Wang, SCAU 000015 (SCAU).

\section{Macroteleia striatipleuron sp. $\mathrm{n}$.}

urn:lsid:zoobank.org:act:53384A4A-28B5-4F08-9CC6-EA8CBBBB5260

http://species-id.net/wiki/Macroteleia_striatipleuron

Plates 62-63

Description. Female. Body length $6.50 \mathrm{~mm}$.

Color. Body black; mandible dark brown; palpi yellow; legs yellow with hind tarsi brown; A1 yellow, A2-A4 brown, A5 dark brown, remainder of antenna black; fore wing subhyaline with medial longitudinal infuscate streak in basal half.

Head. Transverse in dorsal view, width $1.46 \times$ length, as wide as mesosoma; OOL short, $0.25 \times$ minimum ocellar width; POL $1.73 \times$ LOL; occipital carina continuous medially, coarsely and densely punctate; central keel well developed, extending onto interantennal process, slightly bifurcating dorsally (Plate 2A); medial frons smooth dorsally, obliquely strigose ventrally; remainder of frons and vertex punctate reticulate; gena punctate rugose; length of A3 1.03× length of A2.

Mesosoma. Cervical pronotal area punctate reticulate; dorsal pronotal area areolate; lateral pronotal area anteriorly with several carinae subparallel to vertical epomial 


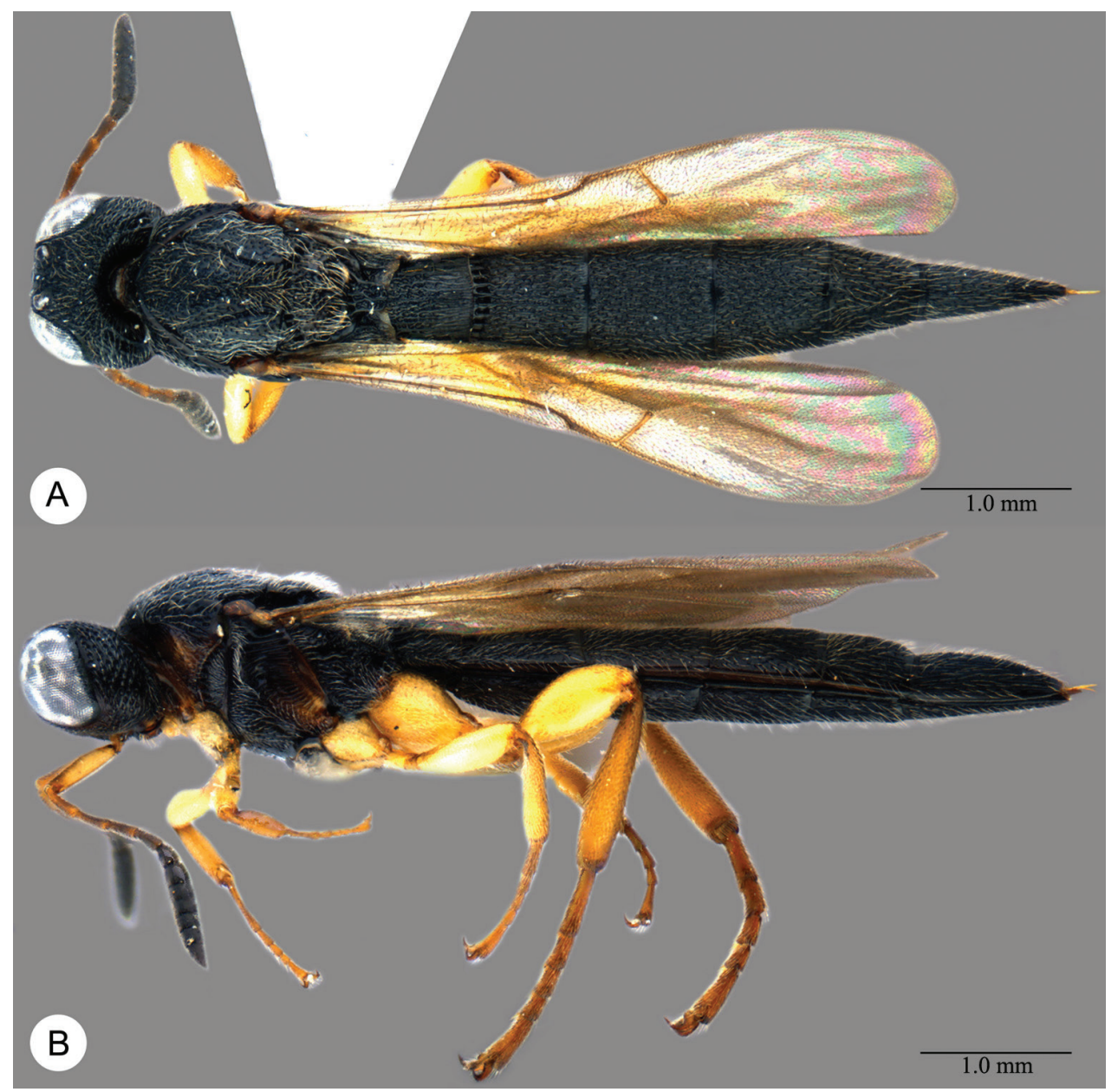

Plate 62. Macroteleia striatipleuron sp. n., holotype, female. A Dorsal habitus B Lateral habitus.

carina, otherwise smooth with sparse punctures; netrion finely punctate rugulose; notaulus deep, distinctly foveolate; middle lobe of mesoscutum densely punctate, becoming punctate reticulate anteriorly and posteriorly; lateral lobes of mesoscutum densely punctate throughout; mesoscutellum densely punctate throughout; metascutellum rectangular (Plate 63A), posterior margin convex, extending to gap between propodeal lobes, irregularly areolate rugose; propodeum medially divided into two widely separated subtriangular lobes (Plate 63B), each side with rugose sculpture covered by dense, recumbent, white setae; upper mesepisternum with a row of robust longitudinal carinae below subalar pit; lower mesepisternum densely punctate; mesopleural depression longitudinally striate (Plate 63B); metapleuron punctate rugose throughout.

Legs. Robust; hind femur strongly swollen, $2.70 \times$ as long as its maximum width; hind tibia without spines over outer surface; hind basitarsus $3.75 \times$ as long as its maximum width. 


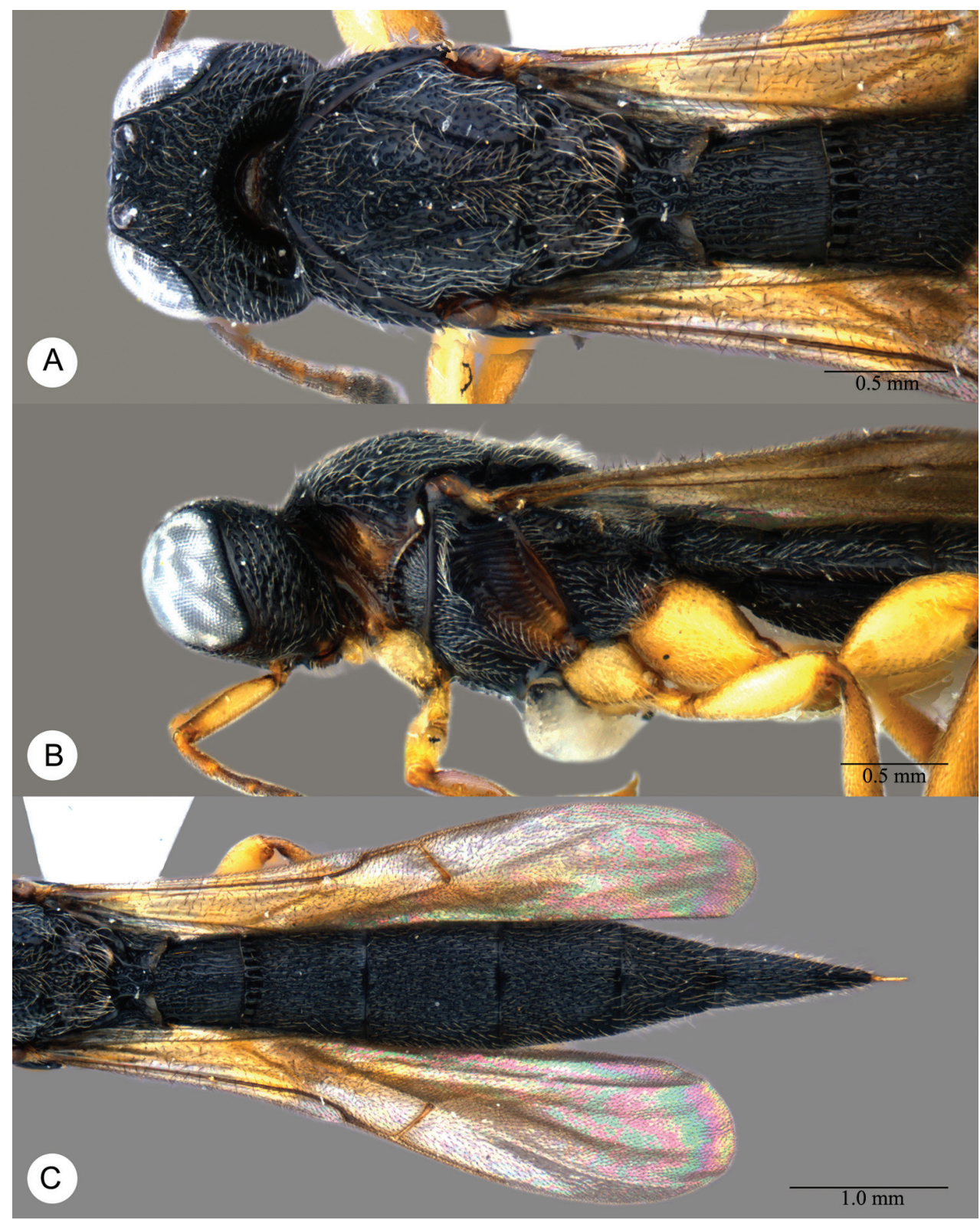

Plate 63. Macroteleia striatipleuron sp. n., holotype, female. A Head and mesosoma, dorsal view B Head and mesosoma, lateral view C Metasoma, dorsal view.

Wings. Apex of fore wing extending as far as mid-length of T6; R $1.36 \times$ as long as r-rs, R1 $1.89 \times$ length of R.

Metasoma. Posterior margin of transverse sulcus on T2 slightly convex (Plate 63C); sublateral tergal carinae developed on T1-T2; T1 longitudinally punctate rugose anteriorly, densely striate posteriorly; T2-T5 longitudinally punctate rugulose through- 
out; T6 longitudinally striate throughout, with scattered small punctures in interstices; length of T3 $0.81 \times$ length of T6; T5 slightly wider than long; S2-S6 densely longitudinally striate, with coarse punctures in interstices; prominent longitudinal median carina strongly developed on S2-S4.

Male. Unknown.

Diagnosis. Macroteleia striatipleuron shares the well-developed central keel, rectangular metascutellum and robust legs with $M$. salebrosa, but can be distinguished by the medially continuous occipital carina (interrupted medially in $M$. salebrosa); longitudinally striate mesopleural depression (smooth in M. salebrosa); and yellow hind coxa (dark brown to nearly black in $M$. salebrosa).

Etymology. The name striatipleuron refers to the longitudinal striae on mesopleural depression of this species and is used as a noun in apposition.

Distribution. China (Guangdong). Link to distribution map [http://hol.osu.edu/ map-large.html?id=320509].

Material examined. Holotype, $q$ : CHINA: Guangdong, Nanling National Nature Reserve, $24^{\circ} 54^{\prime} \mathrm{N}, 113^{\circ} 00^{\prime} \mathrm{E}, 8-17 . \mathrm{VIII} .2010$, sweeping, Huayan Chen, SCAU 000013 (deposited in SCAU).

\section{Macroteleia striativentris Crawford}

http://species-id.net/wiki/Macroteleia_striativentris

Plates 64-69

Macroteleia striativentris Crawford 1910: 126 (original description); Kieffer 1913b: 323 (description, keyed); Kieffer 1914b: 298 (keyed); Kieffer 1926: 521, 527 (description, keyed); Baltazar 1966: 184 (listed, synonymy); Masner and Muesebeck 1968: 40 (type information); Lê 2000: 53, 63 (description, keyed).

Description. Female. Body length 4.06-6.34 mm ( $\mathrm{n}=20)$.

Color. Head and mesosoma black, metasoma dark brown to black; mandible dark brown; palpi yellow; legs pale brown throughout; A1 brown, A2-A5 dark brown, remainder of antenna black; fore wing hyaline.

Head. Transverse in dorsal view, $1.28-1.48 \times$ as wide as long, slightly wider than mesosoma; OOL short, 0.29-0.33× minimum ocellar width; POL 1.25-1.33× LOL; occipital carina interrupted medially; central keel absent or weakly developed above interantennal process (Plates 66C, 68A); medial frons punctate rugulose ventrally, irregularly smooth dorsally; ventrolateral frons punctate rugose; frons below median ocellus densely punctate; vertex densely punctate; gena punctate rugose; length of A3 $0.83-0.90 \times$ length of A2.

Mesosoma. Cervical pronotal area densely punctate; dorsal pronotal area areolate; lateral pronotal area smooth anteriorly, punctate rugulose posteriorly; netrion punctate rugose; notaulus narrow, irregularly foveolate; middle lobe of mesoscutum densely punctate, becoming denser anteriorly and posteriorly; lateral lobes of mes- 


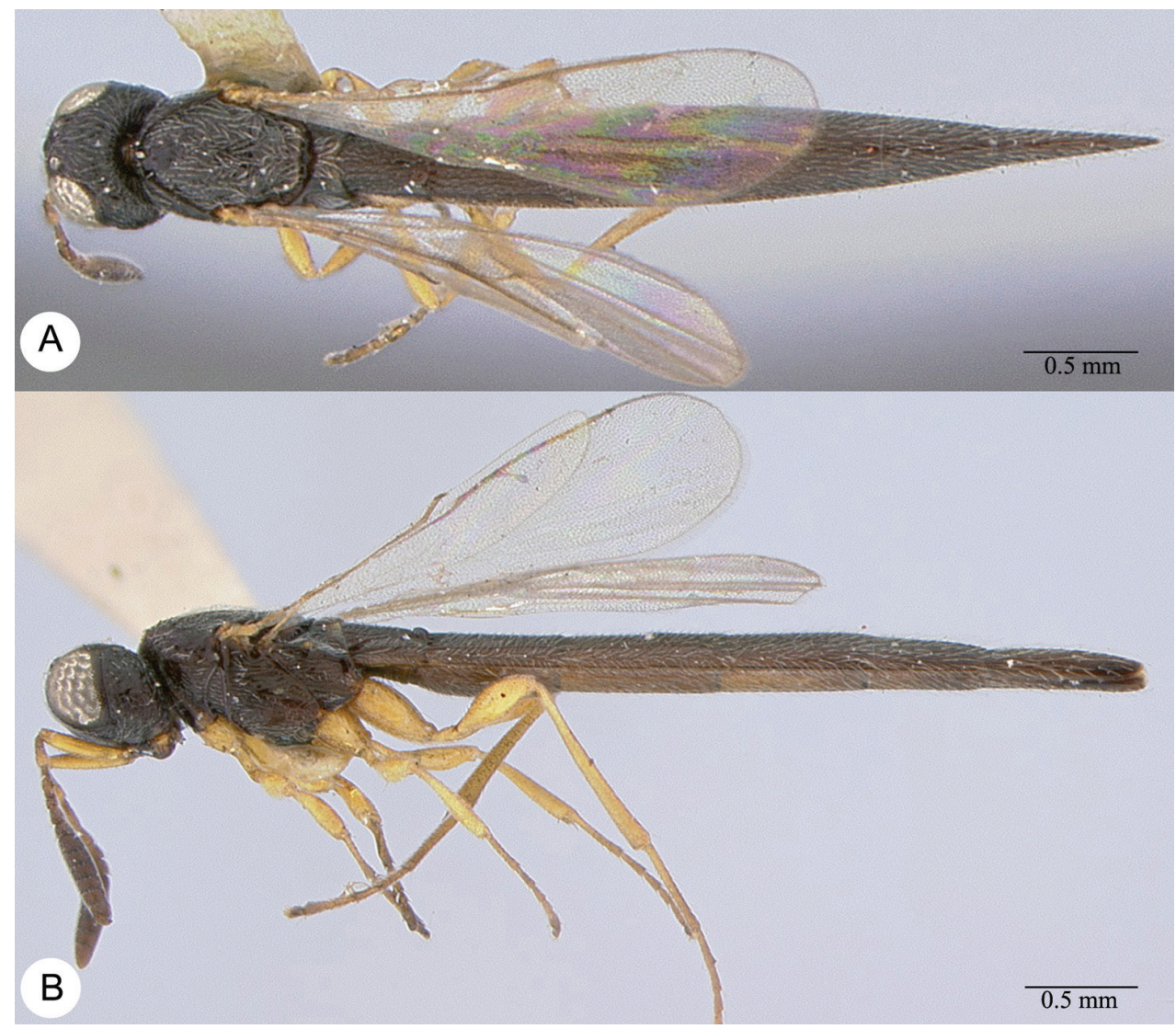

Plate 64. Macroteleia striativentris Crawford, holotype, female. A Dorsal habitus B Lateral habitus.

oscutum densely punctate throughout; mesoscutellum densely punctate throughout; metascutellum distinctly transverse (Plates 65B, 66D, 68B), posterior margin slightly pointed medially, longitudinally carinate; propodeum continuous medially (Plates 65B, 66D, 68B), not divided into two separated lobes, posterior margin narrowly notched medially, each side with several irregular longitudinal carinae medially, otherwise punctate rugulose, covered by dense, recumbent, white setae; upper mesepisternum with a row of robust longitudinal carinae below subalar pit; lower mesepisternum punctate rugulose; mesopleural depression smooth (Plates 65C, 66E, 68C); metapleuron longitudinally striate throughout.

Legs. Slender; hind femur weakly swollen, 4.12-4.87x as long as its maximum width; hind tibia without spines over outer surface; hind basitarsus $10.60-12.50 \times$ as long as its maximum width.

Wings. Apex of fore wing extending as far as mid-length of T4; R 1.33-2.08× as long as r-rs, R1 1.90-2.38× length of R.

Metasoma. Posterior margin of transverse sulcus on T2 straight (Plates 65D, 66F, 68D); sublateral tergal carinae developed on T1-T3; T1 densely longitudinally striate 


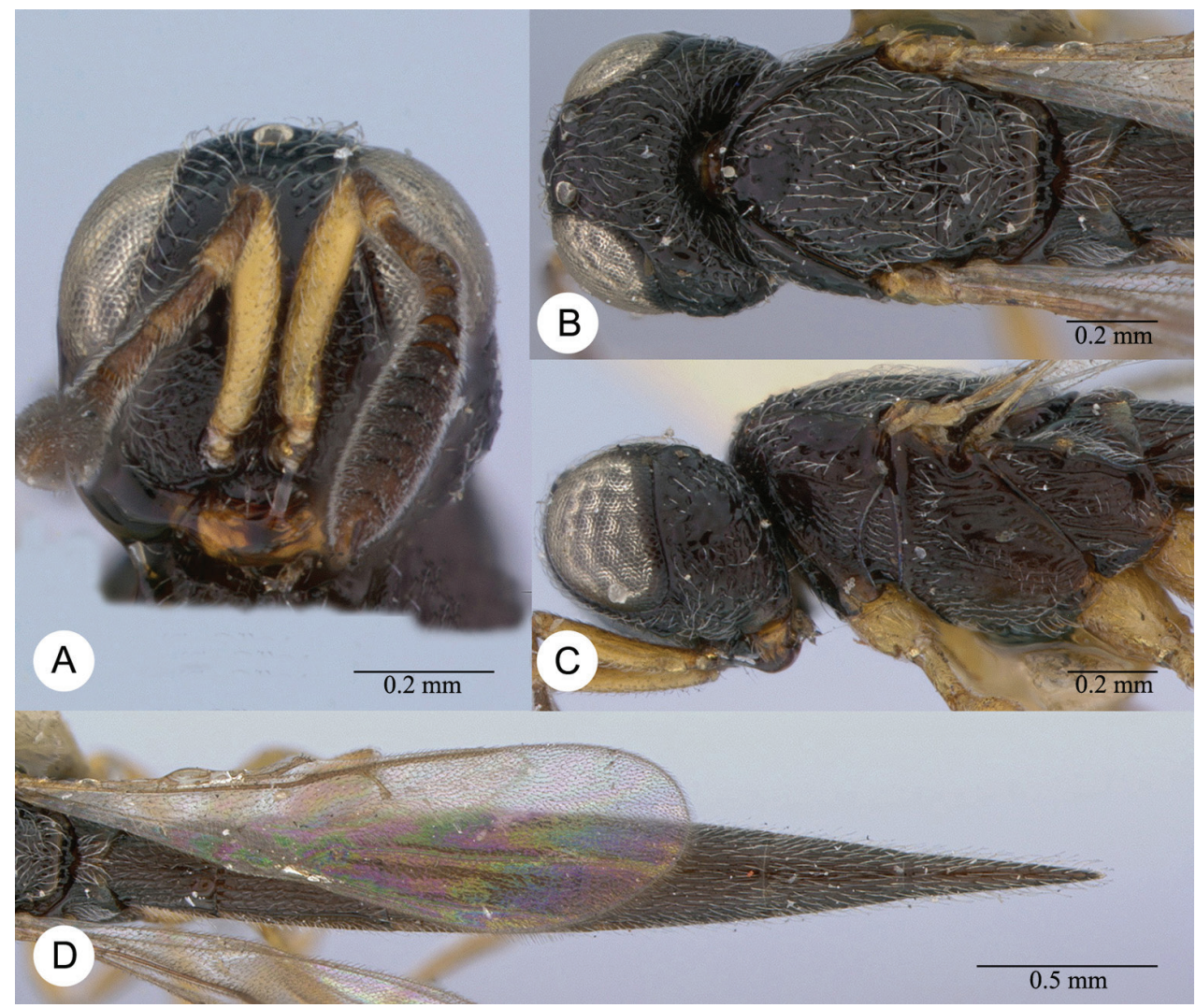

Plate 65. Macroteleia striativentris Crawford, holotype, female. A Head, anterior view B Head and mesosoma, dorsal view C Head and mesosoma, lateral view D Metasoma and fore wing, dorsal view.

medially, with scattered punctures in interstices anteriorly, punctate rugulose laterally; T2-T4 densely longitudinally striate medially, with delicate punctures in interstices, punctate rugulose laterally; T5-T6 finely punctate dorsally, densely longitudinally striate laterally, with scattered small punctures in interstices; length of T3 $0.72-0.97 \times$ length of T6; T5 distinctly longer than wide; S2-S3 sparsely longitudinally striate, with finely punctate rugulose interstices; S4-S6 longitudinally striate, with finely punctate interstices; prominent longitudinal median carina present on S2-S5.

Male. Differing from female as follows: body length 5.22-5.57 $\mathrm{mm}(\mathrm{n}=9)$; head brown to dark brown; mesosoma black; metasoma variably brown to black; A1 brown, remainder of antenna dark brown to black; T1 densely longitudinally striate medially, with rugulose sculpture in interstices anteriorly, punctate rugulose laterally; T2-T3 densely longitudinally striate medially, with delicate punctures in interstices, punctate rugulose laterally; T4-T7 longitudinally punctate rugulose; T6 distinctly longer than wide; length of T6 1.20-1.41× length of T7; T7 subtriangular, apex pointed (Plate 69B); length of T7 2.21-2.50x length of S7; S2-S5 longitudinally striate, with delicate punctures in interstices; S6-S7 longitudinally punctate rugulose. 


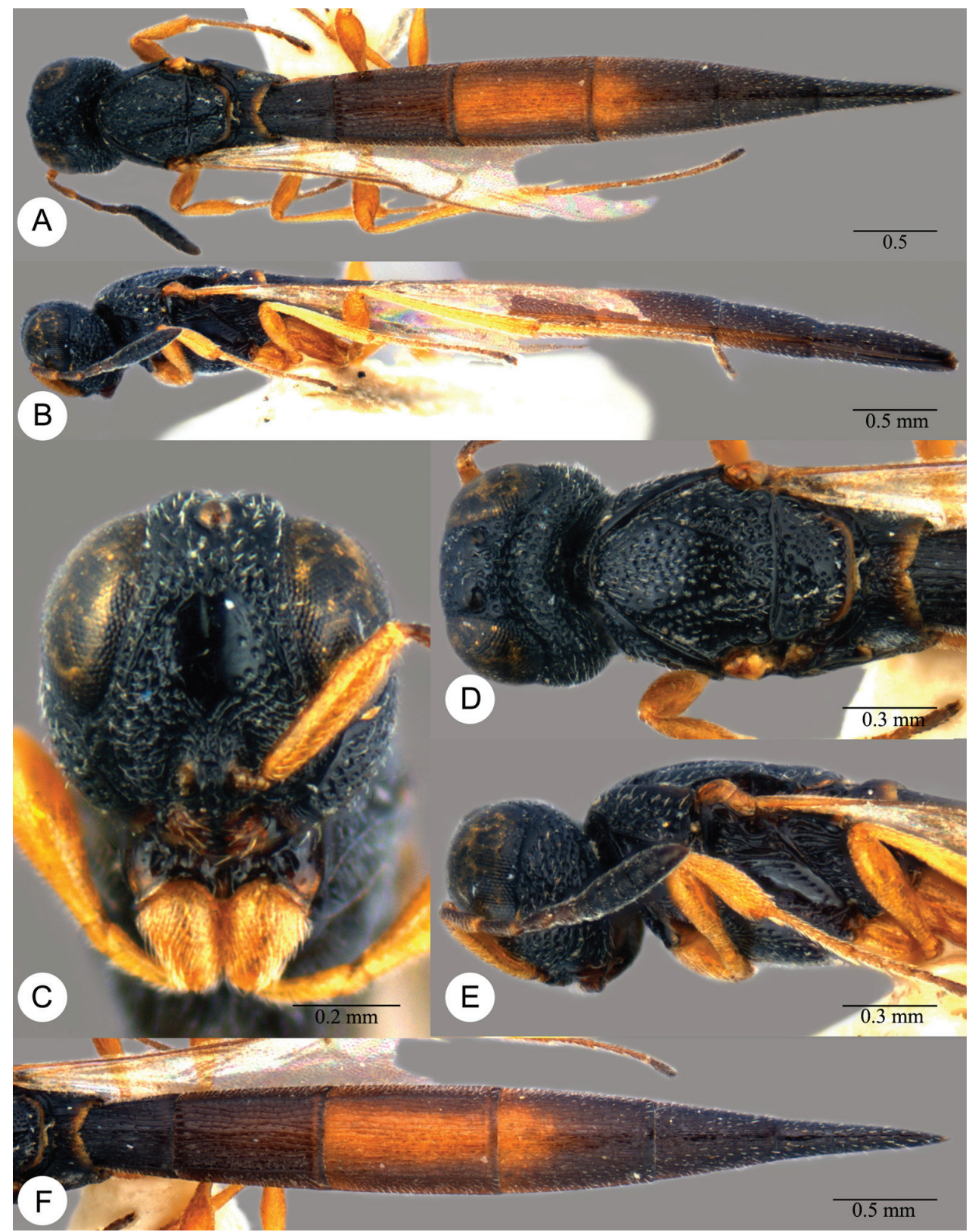

Plate 66. Macroteleia fugacious Kozlov \& Lê, paratype, female. A Dorsal habitus B Lateral habitus C Head, anterior view D Head and mesosoma, dorsal view E Head and mesosoma, lateral view F Metasoma, dorsal view.

Distribution. China (Guangdong, Hainan, Yunnan); Vietnam; Thailand; Philippines. Link to distribution map [http://hol.osu.edu/map-large.html?id=4873].

Material examined. Lectotype, $q$, PHILIPPINES: "Manila PI", "Robt Brown Collector", "Macroteleia striativentris + Type Crawford", "Lectotype + Macroteleia 


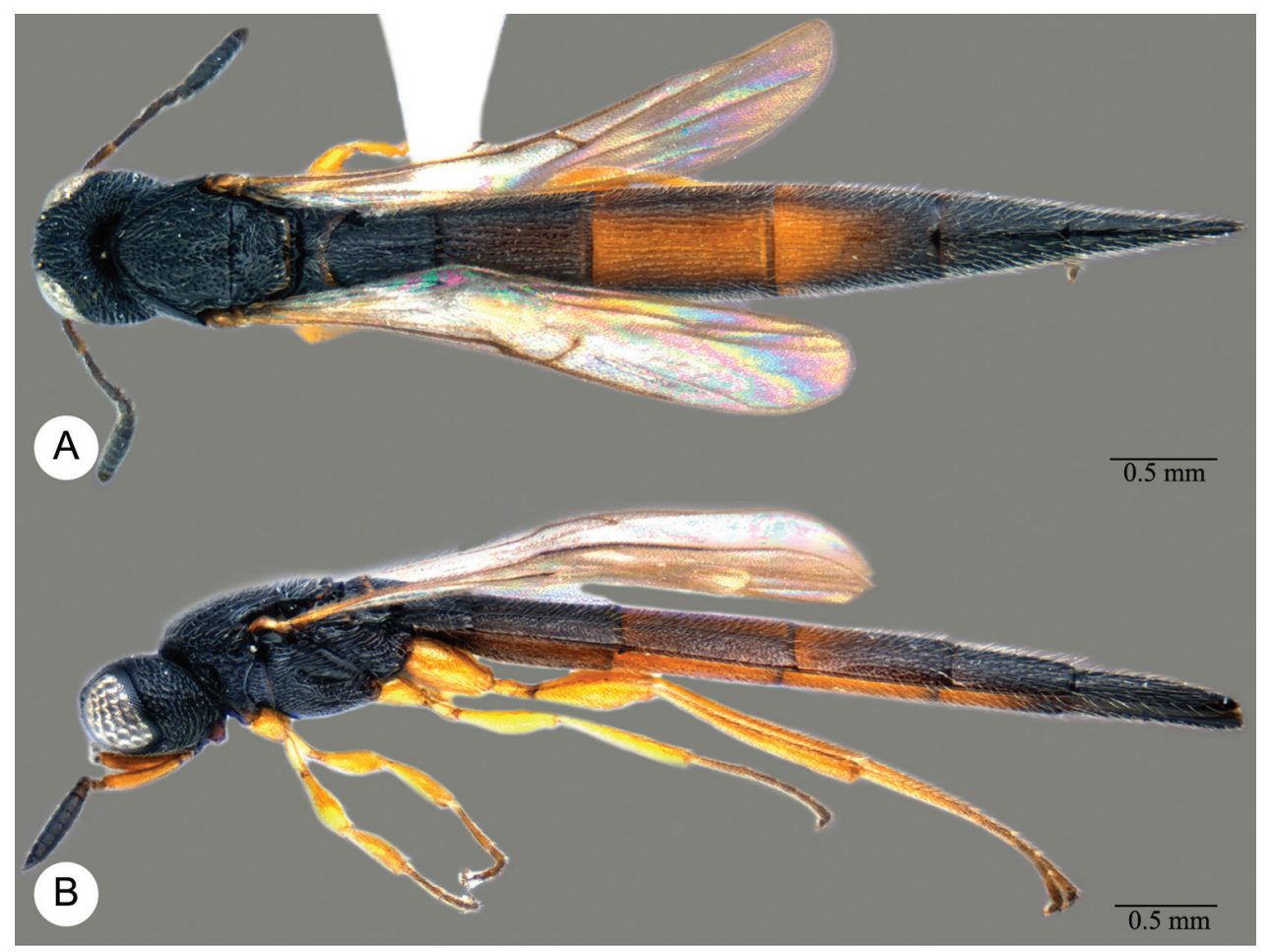

Plate 67. Macroteleia striativentris Crawford, female from Guangdong, Chebaling National Nature Reserve. A Dorsal habitus B Lateral habitus.

striativentris Crawford by L. Masner, 1967”, “Type No 12896 U.S.N.M.” (deposited in USNM).

Other material. CHINA: 1 , Guangdong, Chebaling National Nature Reserve, $24^{\circ} 43^{\prime} \mathrm{N}, 14^{\circ} 14^{\prime} \mathrm{E}, 25 . V .2002$, Jingxian Liu, SCAU 000086 (SCAU); 1 ô, Guang-

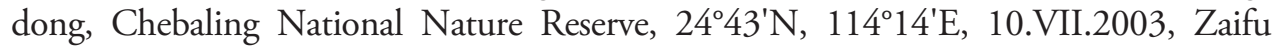
Xu, SCAU 000087 (SCAU); 2 q, Guangdong, Fengkai County, Mt. Qiancengfeng, $23^{\circ} 28^{\prime} \mathrm{N}, 111^{\circ} 48^{\prime} \mathrm{E}, \mathrm{VIII} .2003$, Jingxian Liu, SCAU 000088, 000089 (SCAU); 3 \%, Guangdong, Zijin County, Linjiang Town, 2339'N, 114²1'E, 1.VIII.2003, Jingxian Liu, SCAU 000090-000092 (SCAU); 1 ㅇ, Guangdong, Mt. Tongleda, 2310'N, $111^{\circ} 25^{\prime} \mathrm{E}, 12 . V I I I .2003$, Jujian Chen, SCAU 000093 (SCAU); 4 ㅇ, Guangdong, Zhaoqing, Xiwanggu, $23^{\circ} 13^{\prime} \mathrm{N}, 112^{\circ} 31^{\prime} \mathrm{E}, 2-6 . V I I I .2010$, sweeping, Huayan Chen, SCAU 000094-000097 (SCAU); 3 q, Guangdong, Zhaoqing, Xiwanggu, 2313'N, 112 31'E, 2-6.VIII.2010, yellow pan trap, Huayan Chen, SCAU 000098-000100 (SCAU); 1

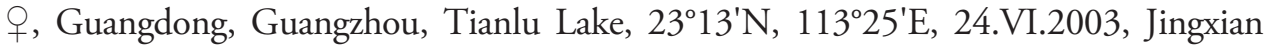
Liu, SCAU 000101 (SCAU); 8 ふ̋, Guangdong, Guangzhou, Tianlu Lake, 2313'N, $113^{\circ} 25^{\prime} \mathrm{E}, 6 . X .2002$, Zaifu Xu, SCAU 000102-000109 (SCAU); 1 q, Hainan, Wuzhishan National Nature Reserve, $18^{\circ} 51^{\prime} \mathrm{N}, 109^{\circ} 39^{\prime} \mathrm{E}, 16-18 . \mathrm{V} .2007$, Jingxian Liu, SCAU 000110 (SCAU); 1 q, Hainan, Mt. Yinggeling, 1849'N, 109¹1'E, 18.X.2007, Jingxian 


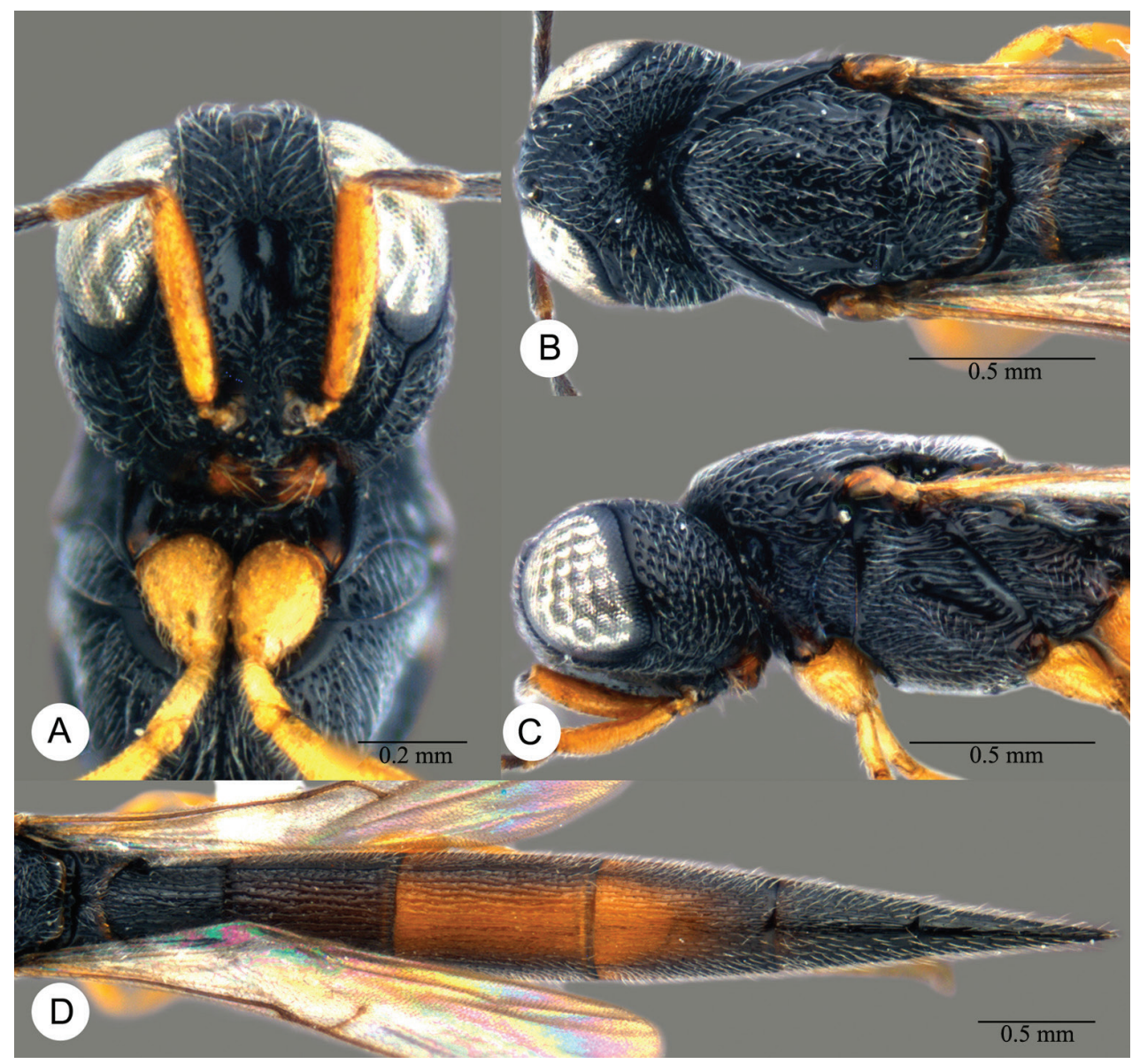

Plate 68. Macroteleia striativentris Crawford, female from Guangdong, Chebaling National Nature Reserve. A Head, anterior view B Head and mesosoma, dorsal view C Head and mesosoma, lateral view D Metasoma, dorsal view.

Liu, SCAU 000111 (SCAU); 3 ㅇ, Hainan, Mt. Yinggeling, 1849’N109¹1'E, 17-20. VII.2010, Huayan Chen, SCAU 000112-000114 (SCAU); 1 + , Yunnan, Lushui County, Shangiiang Town, 2539'N, 9852'E, 19.VII.2006, Jie Zeng, SCAU 000115 (SCAU); 2 , Yunnan, Gaoligongshan National Nature Reserve, $24^{\circ} 49^{\prime} \mathrm{N}, 98^{\circ} 46^{\prime} \mathrm{E}, 1 . \mathrm{VIII} .2005$, Juanjuan Ma, SCAU 000116, 000117 (SCAU). THAILAND: 1 9 , Chiang Mai: Maerim, 10.IV.2003, FIT, R. A. Beaver, No. 27042 (RABC); 1 , Chiang Mai: Maerim, 7.X.2003, FIT, R. A. Beaver, No. 28207 (RABC). VIETNAM: "Bac Thai, Phu Luong, Quan Chu, 23.IV.1986, A. Sarkov", "Paratypus, Macroteleia fugacious sp. n.” (IEBR).

Comments. M. fugacious was described by Lê (2000) based on five females and three males. He stated the holotype (female) and four paratypes (two females and two males) were deposited in the Institute of Ecology and Biological Resources (IEBR). Two other paratypes are deposited in the Zoological Institute in St. Petersburg. How- 


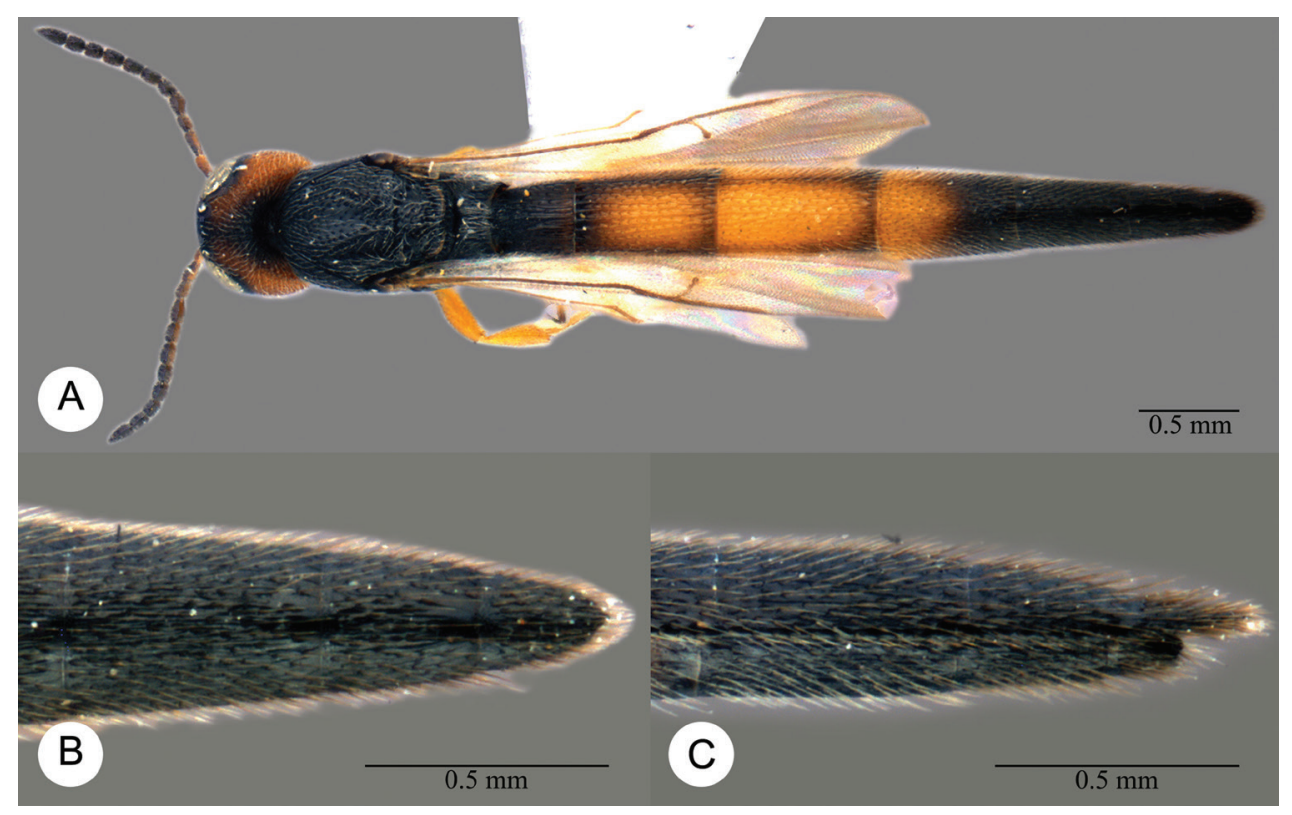

Plate 69. Macroteleia striativentris Crawford, male from Guangdong, Guangzhou, Tianlu Lake. A Dorsal habitus B Apex of metasoma, dorsal view C Apex of metasoma, lateral view.

ever, the second author found that the holotype of $M$. fugacious is housed in the collection of the Zoological Institude in St. Petersburg. When examining the types of Lê's species of Scelioninae in IEBR, the first author only found one female and one male specimens labeled as paratypes of $M$. fugacious. The two paratypes, unfortunately, are different species: the male paratype belongs to $M$. dolichopa, and the female paratype belongs to M. striativentris. We were unable to examine the holotype of M. fugacious in this study, and therefore the identity of $M$. fugacious is uncertain.

\section{Acknowledgements}

Thanks to Dr Lê Xuan Hue and Dr Khuat Dang Long (IEBR) for the loan of Vietnamese Macroteleia types; to Dr Nguyen Duc Tung (Hanoi University of Agriculture) for his kind help during the study of the Vietnamese Macroteleia species; to Dr R.A. Beaver (Thailand) for the loan of his specimens; to Dr Ovidiu A. Popovici (University 'Al. I. Cuza' Iaşi) for discussion; to M. Buffington and T. Nuhn for loans of the types deposited in Washington; to Prof. Junhua He and Prof. Xuexin Chen of Zhejiang University for their generous loan of their specimens of Macroteleia; and to Jingxian Liu, Liqiong Weng, Long Hu, Bin Xiao, Huiting Chen, Yali Cai, Chundan Hong, Jie Zeng, Juanjuan Ma, Jiemin Yao, and Chengyuan Jin for their help during field trips. This material is based upon work supported in part by the National Basic Research Program of China (No. 2013CB127600) and the National Science Foundation of USA under grant No. DEB-0614764 to N.F. Johnson and A.D. Austin. 


\section{References}

Ashmead WH (1893) A monograph of the North American Proctotrypidae. Bulletin of the United States National Museum 45: 1-472. doi: 10.5479/si.03629236.45.1

Ashmead WH (1894) Report on the parasitic Cynipidae, part of the Braconidae, the Ichneumonidae, the Proctotrypidae, and part of the Chalcidinae. Part III. Zoological Journal of the Linnean Society of London 25: 188-254.

Ashmead WH (1900) Report upon the aculeate Hymenoptera of the islands of St. Vincent and Grenada, with additions to the parasitic Hymenoptera and a list of the described Hymenoptera of the West Indies. Transactions of the Royal Entomological Society of London 1900: 207-367

Ashmead WH (1903) Classification of the pointed-tailed wasps, or the superfamily Proctotrypoidea. - III. Journal of the New York Entomological Society 11: 86-99.

Austin AD, Field SA (1997) The ovipositor system of scelionid and platygastrid wasps (Hymenoptera: Platygastroidea): comparative morphology and phylogenetic implications. Invertebrate Taxonomy 11: 1-87. doi: 10.1071/IT95048

Baltazar CR (1966) A catalogue of Philippine Hymenoptera (with a bibliography, 1758-1963). Pacific Insects Monographs 8: 1-488.

Brues CT (1907) Notes and descriptions of North American parasitic Hymenoptera. V. Bulletin of the Wisconsin Natural History Society 5: 150-161.

Brues CT (1908) Hymenoptera. Fam. Scelionidae. Genera Insectorum 80: 1-59.

Brullé A (1846) Histoire naturelle des insects. Hyménoptères. Tome quatrième. Librairie Encyclopédique de Roret, Paris. 680 pp.

Carpenter FM (1992) Arthropoda 4. Superclass Hexopoda. Treatise on Invertebrate Paleontology, Part R. The Geological Society of America, Boulder, CO. 655 pp.

Cole AC (1931) Typha insects and their parasites. Entomological News 42: 35-39.

Crawford JC (1910) New Hymenoptera from the Philippine Islands. Proceedings of the U.S. National Museum 38: 119-133.

Cresson ET (1887) Synopsis of the families and genera of the Hymenoptera of America, north of Mexico, together with a catalogue of the described species, and a bibliography. Transactions of the American Entomological Society Suppl.: 1-351.

Dalla Torre CG de (1898) Catalogus hymenopterorum hucusque descriptiorum systematicus et synonymicus. Vol. V: Chalcididae et Proctotrupidae. Sumptibus Guilelmi Engelmann, Lipsiae. 598 pp.

De Santis L (1967) Catálogo de los himenópteros argentinos de la serie parasitica, incluyendo Bethyloidea. Com. Inv. Cient., Pcia Buenos Aires Gob., La Plata. 337 pp.

De Santis L (1980) Catálogo de los himenópteros brasileños de la serie Parasitica incluyendo Bethyloidea. Editora da Universidade Federal do Paraná, Curitiba, Brazil. 395 pp.

Dodd AP (1913a) Australian Hymenoptera Proctotrypoidea. No. 1. Transactions of the Royal Society of South Australia 37: 130-181.

Dodd AP (1913b) Some new parasitic Hymenoptera from Australia. Archiv für Naturgeschichte 79(6): 164-182. 
Dodd AP (1915) Notes and corrections on Australian Proctotrypoidea, with descriptions of forty-five new species. Archiv für Naturgeschichte 80(9): 1-32.

Dodd AP (1920) Notes on the exotic Proctotrupoidea in the British and Oxford University Museums, with descriptions of new genera and species. Transactions of the Entomological Society of London 1919: 321-382.

Dodd AP (1933) The Australian species of Macroteleia and Prosapegus (Scelionidae) Hymenoptera. Proceedings of the Royal Society of Queensland 44: 75-103.

Förster A (1856) Hymenopterologische Studien. II. Heft. Chalcidae und Prototrupii. Ernst ter Meer, Aachen. 152 pp.

Fouts RM (1930) New bethylid and serphoid parasites from Borneo and the Philippine Islands. Philippine Journal of Science 41: 1-11.

Galloway ID (1978) A revision of the Australian species of Macroteleia Westwood (Hymenoptera: Scelionidae: Scelioninae). Journal of the Australian Entomological Society 17: 297-310. doi: 10.1111/j.1440-6055.1978.tb01494.x

Galloway ID, Austin AD (1984) Revision of the Scelioninae (Hymenoptera: Scelionidae) in Australia. Australian Journal of Zoology Supplementary Series 99: 1-138.

Howard LO (1886) A generic synopsis of the hymenopterous family Proctotrupidae. Transactions of the American Entomological Society 13: 169-178.

Johnson NF (1992) Catalog of world Proctotrupoidea excluding Platygastridae. Memoirs of the American Entomological Institute 51: 1-825.

Johnson NF (2012) Platygastroidea Online. [01/06/2012]. http://osuc.biosci.ohio-state.edu/ hymDB/eol_scelionidae.home

Kieffer JJ (1908a) Nouveaux proctotrypides et cynipides d'Amerique recueillis par M. Baker. Annales de la Société Scientifique de Bruxelles. Mémoires 32: 7-64.

Kieffer JJ (1908b) Revision des Scelionidae (Hyménoptères). Annales de la Société Scientifique de Bruxelles. Mémoires 32: 111-250.

Kieffer JJ (1910a) Description de nouveaux microhyménoptères du Brésil. Annales de la Société Entomologique de France 78: 287-348.

Kieffer JJ (1910b) Hymenoptera. Fam. Scelionidae. Addenda et corrigenda. Genera Insectorum 80: 61-112.

Kieffer JJ (1912) Hymenoptera, Proctotrupoidea. Transactions of the Linnean Society of London, Zoology 15: 45-80.

Kieffer JJ (1913a) Proctotrypidae (3e partie). Species des Hyménoptères d'Europe et d'Algerie 11: 161-304.

Kieffer JJ (1913b) Serphides des Îles Philippines. Insecta 3: 253-462.

Kieffer JJ (1914a) Proctotrypidae (3e partie). Species des Hymenopteres d'Europe et d'Algerie 11: 305-448.

Kieffer JJ (1914b) Énumeration des Serphides (Proctotrupides) des Îles Philippines avec description de genres nouveaux et d'espèces nouvelles. Philippine Journal of Science (D) 9: 285-311.

Kieffer JJ (1917) Neue Scelioniden aus den Philippinen-Inseln. Brotéria 15: 50-62.

Kieffer JJ (1926) Scelionidae. Das Tierreich. Vol. 48. Walter de Gruyter \& Co., Berlin, 885 pp. 
Kononova SV (1995) [Fam. Scelionidae.] Pages 57-121 in Lehr PA. [Key to insects of Russian Far East in six volume. vol. 4. Neuropteroidea, Mecoptera, Hymenoptera. Part 2. Hymenoptera. Dal'nauka, Vladivostok. 600 pp.

Kononova SV, Kozlov MA (2008) [Scelionids of the Palearctic (Hymenoptera, Scelionidae). Subfamily Scelioninae. Tovarishchestvo Nauchnykh Izdanii KMK, Saint Petersburg. 489 pp.

Kononova SV, Petrov S (2003) [New species of egg parasites of the family Scelionidae (Hymenoptera, Proctotrupoidea) of the Palearctic fauna.] Zoologicheskii Zhurnal 82: 603-612.

Kozlov MA (1971) [Proctotrupoids (Hymenoptera, Proctotrupoidea) of the USSR.] Trudy Vsesoyuznogo Entomologicheskogo Obshchestva 54: 3-67.

Kozlov MA (1978) [Superfamily Proctotrupoidea]. Pages 538-664 in Medvedev GS [Determination of insects of the European portion of the USSR.] Vol. 3, part 2. Nauka, Leningrad. 758 pp.

Kozlov MA, Kononova SV (1987) [New Palearctic species of the genus Macroteleia Westwood, 1835 (Hymenoptera, Scelionidae, Scelioninae).] Pages 93-101 in Ler P A, Storozheva NA. [New data on the systematics of insects of the Far East.] Acad. Sci. USSR, Far East Branch, Biology \& Soil Institute, Vladivostok. 144 pp.

Kozlov MA, Kononova SV (1990) [Scelioninae of the Fauna of the USSR (Hymenoptera, Scelionidae, Scelioninae).] Nauka, Leningrad. 344 pp.

Lê XH (2000) Egg-parasites of family Scelionidae (Hymenoptera). Fauna of Vietnam, vol. 3. Science and Technics Publishing House, Hanoi. 386 pp.

Loiácono MS, Margaría CB (2002) Systematics, morphology and physiology. Ceraphronoidea, Platygastroidea and Proctotrupoidea from Brazil (Hymenoptera). Neotropical Entomology 31(4): 551-560. doi: 10.1590/S1519-566X2002000400007

Maneval H (1940) Fam. XVII. Proctotrypides. Pages 93-118 in Perrier R. La Faune de la France en tableaux synoptiques illustrés. Tome VII. Hyménoptères par Lucien Berland avec la collaboration de MM. Raymond Benoit, Francis Bernard, Henri Maneval. Paris. 213 pp. Mani MS, Sharma SK (1982) Proctotrupoidea (Hymenoptera) from India. A review. Oriental Insects 16: 135-258. doi: 10.1080/00305316.1982.10434314

Masner L (1956) Bemerkungen zur Systematik der Gattung Parapegus Kieffer, 1908 (Hym. Scelionidae). Zoologischer Anzeiger 157(11/12): 234-239.

Masner L (1964) A comparison of some Nearctic and Palearctic genera of Proctotrupoidea (Hymenoptera) with revisional notes. Časopis Československé Společnosti Entomologické 61: 123-155.

Masner L (1965) The types of Proctotrupoidea (Hymenoptera) in the British Museum (Natural History) and in the Hope Department of Entomology, Oxford. Bulletin of the British Museum (Natural History) Entomology Supplement 1: 1-154.

Masner L (1976) Revisionary notes and keys to world genera of Scelionidae (Hymenoptera: Proctotrupoidea). Memoirs of the Entomological Society of Canada 97: 1-87. doi: $10.4039 /$ entm $10897 \mathrm{fv}$

Masner L (1980) Key to genera of Scelionidae of the Holarctic region, with descriptions of new genera and species (Hymenoptera: Proctotrupoidea). Memoirs of the Entomological Society of Canada 113:1-54. doi: 10.4039/entm112113fv 
Masner L, Muesebeck CFW (1968) The types of Proctotrupoidea (Hymenoptera) in the United States National Museum. Bulletin of the United States National Museum 270: 1-143. doi: 10.5479/si.03629236.270

Mikó I, Vilhelmsen L, Johnson NF, Masner L, Pénzes Z (2007) Skeletomusculature of Scelionidae (Hymenoptera: Platygastroidea): head and mesosoma. Zootaxa 1571: 1-78.

Morgan HA (1901) The differential grasshopper in the Mississippi Delta - other common species. Bulletin of the United States Department of Agriculture, Division of Entomology (New Series) 30: 7-33.

Muesebeck CFW (1977) The parasitic wasps of the genus Macroteleia Westwood of the New World (Hymenoptera, Proctotrupoidea, Scelionidae). U.S. Department of Agriculture Technical Bulletin 1565: 1-57.

Muesebeck C F W (1979) Superfamily Proctotrupoidea. Pages 1121-1186 in Krombein KV et al. Catalog of Hymenoptera in America north of Mexico. Smithsonian Institution Press, Washington, DC. 2735 pp.

Muesebeck CFW, Masner L (1967) Superfamily Proctotrupoidea. Pages 285-304 in Krombein KV and Burks BD. Hymenoptera of America north of Mexico. Synoptic Catalog (Agriculture Monograph No. 2). Second supplement. United States Government Printing Office, Washington. 584 pp.

Muesebeck CFW, Walkley LM (1951) Superfamily Proctotrupoidea. Pages 655-718 in Muesebeck CFW, Krombein KV and Townes HK. Hymenoptera of America north of Mexico - Synoptic Catalog. U.S. Dept. Agriculture Monograph No. 2, . 1420 pp.

Muesebeck CFW, Walkley LM (1956) Type species of the genera and subgenera of parasitic wasps comprising the superfamily Proctotrupoidea (order Hymenoptera). Proceedings of the U.S. National Museum 105: 319-419.

Nixon GEJ (1931) On some new South African Proctotrupoidea (Hymenoptera). Eos 7: 355-382.

Nixon GEJ (1933) A further contribution to the study of South Africa Scelionidae (Insecta, Hymenoptera, Proctotrupoidea). Annals and Magazine of Natural History (10)12: 288-563. doi: 10.1080/00222933308655417

Petrov S (1994) Macroteleia angelovi (Hymenoptera, Proctotrupoidea, Scelionidae) - new species from Bulgaria. Second National Scientific Conference of Entomology, 25-27 October 1993, Sofia: 95-97.

Popovici OA, Johnson NF (2012) Gross anatomy of the Malpighian tubules and internal male genitalia of Scelioninae (Hymenoptera; Platygastroidea; Platygastridae) with phylogenetic implications. Proceedings of the Entomological Society of Washington 114: 372-397. doi: 10.4289/0013-8797.114.3.372

Priesner H (1951) New genera and species of Scelionidae (Hymenoptera, Proctotrupoidea) from Egypt. Bulletin de l'Institut Fouad I du Desert 1(2): 119-149.

Provancher L (1888) Additions et corrections au volume II de la faune entomologique du Canada traitant des Hyménoptères. C. Darveau, Québec. 477 pp.

Rajmohana K (2006) Studies on Proctotrupoidea and Platygastroidea (Hymenoptera: Insecta) of Kerala. Memoirs of the Zoological Survey of India 21(1): 1-153. 
Risbec J (1950) Contribution à l'étude des Proctotrupidae (Serphiidae). Pages 511-639 in Risbec J. Travaux du Laboratoire d'Entomologie du Secteur Soudanis de Recherches Agronomiques. Gouvernement Général de l'Afrique Occidentale Française, Paris. 639 pp. Saraswat GG (1982) Some Indian Proctotrupoidea (Hymenoptera: Scelionidae). Records of the Zoological Survey of India 79: 343-358.

Saraswat GG, Sharma SK (1978) On some Scelionidae (Hymenoptera: Proctotrupoidea) from India. Memoirs of the School of Entomology, St. John's College 5: 1-46.

Schulz WA (1906) Spolia Hymenopterologica. Junfermannschen Buchhandlung, Paderborn. $355 \mathrm{pp}$.

Szabó JB (1966) Neue Macroteleia-Arten aus Ungarn (Hymenoptera, Proctotrupoidea, Scelionidae). Annales Historico-Naturales Musei Nationalis Hungarici 58: 421-425.

Szabó JB (1973) Über die mongolische Proctotrupoiden-Fauna (Hymenoptera). I. Acta Zoologica Academiae Scientiarum Hungaricae 19: 171-179.

Szelényi G (1938) Új Macroteleia (Hym. Proct.) Magyarországból. A new species of Macroteleia Westw. (Hymenopt. Proctotrupoidea) from Hungary. Állattani Közlemények 35: 91-94.

Westwood JO (1835) Characters of new genera and species of hymenopterous insects. Proceedings of the Zoological Society of London 3: 51-72.

Yorder MJ, Mikó I, Seltmann KC, BertoneMA, Deans AR (2010) A gross anatomy ontology for Hymenoptera. PLoS ONE 5(12): e15991. doi: 10.1371/journal.pone.0015991 


\section{Appendix}

URI Table matching terms and concepts used in this revision with the Hymenoptera Anatomy Ontology database.

\begin{tabular}{|c|c|c|}
\hline & A1 & http://purl.obolibrary.org/obo/HAO_0000908 \\
\hline & A2 & http://purl.obolibrary.org/obo/HAO_0000706 \\
\hline & A3 & http://purl.obolibrary.org/obo/HAO_0001148 \\
\hline & A7 & http://purl.obolibrary.org/obo/HAO_0001885 \\
\hline & A12 & http://purl.obolibrary.org/obo/HAO_0001884 \\
\hline & antenna & http://purl.obolibrary.org/obo/HAO_0000101 \\
\hline & antennomere & http://purl.obolibrary.org/obo/HAO_0000107 \\
\hline & area & http://purl.obolibrary.org/obo/HAO_0000146 \\
\hline & body & http://purl.obolibrary.org/obo/HAO_0000182 \\
\hline & carina & http://purl.obolibrary.org/obo/HAO_0000188 \\
\hline & central keel & http://purl.obolibrary.org/obo/HAO_0000109 \\
\hline \multirow[t]{6}{*}{ сра } & cervical pronotal area & http://purl.obolibrary.org/obo/HAO_0000194 \\
\hline & clava & http://purl.obolibrary.org/obo/HAO_0000203 \\
\hline & clypeus & http://purl.obolibrary.org/obo/HAO_0000212 \\
\hline & compound eye & http://purl.obolibrary.org/obo/HAO_0000217 \\
\hline & coxa & http://purl.obolibrary.org/obo/HAO_0000228 \\
\hline & depression & http://purl.obolibrary.org/obo/HAO_0000241 \\
\hline \multirow[t]{16}{*}{ dpa } & dorsal pronotal area & http://purl.obolibrary.org/obo/HAO_0000267 \\
\hline & egg & http://purl.obolibrary.org/obo/HAO_0000286 \\
\hline & epomial carina & http://purl.obolibrary.org/obo/HAO_0000307 \\
\hline & eye & http://purl.obolibrary.org/obo/HAO_0000217 \\
\hline & femur & http://purl.obolibrary.org/obo/HAO_0000327 \\
\hline & fore wing & http://purl.obolibrary.org/obo/HAO_0000351 \\
\hline & frons & http://purl.obolibrary.org/obo/HAO_0001523 \\
\hline & gena & http://purl.obolibrary.org/obo/HAO_0000371 \\
\hline & head & http://purl.obolibrary.org/obo/HAO_0000397 \\
\hline & hind coxa & http://purl.obolibrary.org/obo/HAO_0000587 \\
\hline & hind tibia & http://purl.obolibrary.org/obo/HAO_0000631 \\
\hline & hind wing & http://purl.obolibrary.org/obo/HAO_0000400 \\
\hline & inner orbit & http://purl.obolibrary.org/obo/HAO_0000419 \\
\hline & interantennal process & http://purl.obolibrary.org/obo/HAO_0000422 \\
\hline & lateral lobe of mesoscutum & http://purl.obolibrary.org/obo/HAO_0000466 \\
\hline & lateral ocellus & http://purl.obolibrary.org/obo/HAO_0000481 \\
\hline LOL & lateral ocellar line & http://purl.obolibrary.org/obo/HAO_0000480 \\
\hline \multirow[t]{5}{*}{ lpa } & lateral pronotal area & http://purl.obolibrary.org/obo/HAO_0000483 \\
\hline & malar sulcus & http://purl.obolibrary.org/obo/HAO_0000504 \\
\hline & mandible & http://purl.obolibrary.org/obo/HAO_0000506 \\
\hline & margin & http://purl.obolibrary.org/obo/HAO_0000510 \\
\hline & mesepisternum & http://purl.obolibrary.org/obo/HAO_0001872 \\
\hline \multirow[t]{2}{*}{ med } & mesopleural depression & http://purl.obolibrary.org/obo/HAO_0000326 \\
\hline & mesopleuron & http://purl.obolibrary.org/obo/HAO_0000566 \\
\hline
\end{tabular}




\begin{tabular}{|c|c|c|}
\hline & mesoscutellum & http://purl.obolibrary.org/obo/HAO_0000574 \\
\hline & mesoscutum & http://purl.obolibrary.org/obo/HAO_0001490 \\
\hline & mesosoma & http://purl.obolibrary.org/obo/HAO_0000576 \\
\hline & metapleuron & http://purl.obolibrary.org/obo/HAO_0000621 \\
\hline & metascutellum & http://purl.obolibrary.org/obo/HAO_0000625 \\
\hline & metasoma & http://purl.obolibrary.org/obo/HAO_0000626 \\
\hline & midlobe of mesoscutum & http://purl.obolibrary.org/obo/HAO_0000520 \\
\hline & netrion & http://purl.obolibrary.org/obo/HAO_0000644 \\
\hline & notauli (notaulus) & http://purl.obolibrary.org/obo/HAO_0000647 \\
\hline & occipital carina & http://purl.obolibrary.org/obo/HAO_0000653 \\
\hline & ocellus & http://purl.obolibrary.org/obo/HAO_0000661 \\
\hline ot & ocellar triangle & http://purl.obolibrary.org/obo/HAO_0000430 \\
\hline \multirow[t]{2}{*}{ OOL } & ocular ocellar line & http://purl.obolibrary.org/obo/HAO_0000662 \\
\hline & orbit & http://purl.obolibrary.org/obo/HAO_0000672 \\
\hline \multirow[t]{27}{*}{ POL } & posterior ocellar line & http://purl.obolibrary.org/obo/HAO_0000759 \\
\hline & process & http://purl.obolibrary.org/obo/HAO_0000822 \\
\hline & propodeum & http://purl.obolibrary.org/obo/HAO_0001248 \\
\hline & S1 & http://purl.obolibrary.org/obo/HAO_0001997 \\
\hline & S2 & http://purl.obolibrary.org/obo/HAO_0001829 \\
\hline & S3 & http://purl.obolibrary.org/obo/HAO_0001831 \\
\hline & $S 4$ & http://purl.obolibrary.org/obo/HAO_0001832 \\
\hline & S5 & http://purl.obolibrary.org/obo/HAO_0001833 \\
\hline & S6 & http://purl.obolibrary.org/obo/HAO_0001834 \\
\hline & S7 & http://purl.obolibrary.org/obo/HAO_0002185 \\
\hline & sculpture & http://purl.obolibrary.org/obo/HAO_0000913 \\
\hline & sternite & http://purl.obolibrary.org/obo/HAO_0001654 \\
\hline & sulcus & http://purl.obolibrary.org/obo/HAO_0000978 \\
\hline & T1 & http://purl.obolibrary.org/obo/HAO_0000053 \\
\hline & T2 & http://purl.obolibrary.org/obo/HAO_0000056 \\
\hline & T3 & http://purl.obolibrary.org/obo/HAO_0000057 \\
\hline & T4 & http://purl.obolibrary.org/obo/HAO_0000058 \\
\hline & T5 & http://purl.obolibrary.org/obo/HAO_0000059 \\
\hline & T6 & http://purl.obolibrary.org/obo/HAO_0000060 \\
\hline & T7 & http://purl.obolibrary.org/obo/HAO_0000061 \\
\hline & tergite & http://purl.obolibrary.org/obo/HAO_0001783 \\
\hline & tibia & http://purl.obolibrary.org/obo/HAO_0001017 \\
\hline & tyloid & http://purl.obolibrary.org/obo/HAO_0001199 \\
\hline & vein & http://purl.obolibrary.org/obo/HAO_0001095 \\
\hline & vertex & http://purl.obolibrary.org/obo/HAO_0001077 \\
\hline & vertical epomial carina & http://purl.obolibrary.org/obo/HAO_0000307 \\
\hline & wing & http://purl.obolibrary.org/obo/HAO_0001089 \\
\hline
\end{tabular}

